\title{
DE-IA26-05NT42546
}

\section{Elucidation of Polymer and Polymer Composite Behavior in Molding} Operation

\author{
Final Report
}

\section{National Science Foundation}

Division of Design, Manufacturing and Industrial Innovation 


\section{DISCLAIMER}

This report was prepared as an account of work sponsored by an agency of the United States Government. Neither the United States Government nor any agency thereof, nor any of their employees, makes any warranty, express or implied, or assumes any legal liability or responsibility for the accuracy, completeness, or usefulness of any information, apparatus, product, or process disclosed, or represents that its use would not infringe privately owned rights. Reference herein to any specific commercial product, process, or service by trade name, trademark, manufacturer, or otherwise does not necessarily constitute or imply its endorsement, recommendation, or favoring by the United States Government or any agency thereof. The views and opinions of authors expressed herein do not necessarily state or reflect those of the United States Government or any agency thereof. 
Final Report for Period: 09/2009 - 01/2010

Principal Investigator: Sherwood, James .

Organization: Univ of Mass Lowell

\section{Submitted By:}

Sherwood, James - Principal Investigator

Title:

Linking Process-Induced Properties to Thermoplastic-Matrix Woven-Fabric Composites Performance

\section{Senior Personnel}

\section{Project Participants}

Name: Sherwood, James

Worked for more than 160 Hours: Yes

Contribution to Project:

Prof Sherwood (Mechanical Engineering) is the PI on this project. His primary efforts are in the finite element modeling and friction studies.

Name: Gorbatikh, Larissa

Worked for more than 160 Hours: Yes

Contribution to Project:

Prof Gorbatikh's (Mechanical Engineering) primary involvement is in the solid mechanics analysis of the connection between manufacturing-induced defects and variations (e.g., non-orthogonal fiber orientations and local variations in fiber distribution) on in-service failures and damage tolerance.

Name: Chen, Julie

Worked for more than 160 Hours: Yes

Contribution to Project:

Prof Chen's (Mechanical Engineering) primary involvement is in the experimental studies and analytical modeling of yarn and fabric friction and compaction.

\section{Post-doc}

Name: Liu, Lu

Worked for more than 160 Hours: Yes

Contribution to Project:

Lu completed his doctoral degree in December 2005. He worked on this project while completing his doctoral degree and for a few months beyond that as a postdoc. Lu worked mostly on analytical modeling of fabric deformation and compaction during forming and experimental measurement of yarn-on-yarn friction and compaction. He was funded partially by this grant and partially from an industrial grant.

Name: Jauffres, David

Worked for more than 160 Hours: Yes

Contribution to Project:

\section{Graduate Student}

Name: Gamache, Lisa

Worked for more than 160 Hours: Yes

Contribution to Project:

Lisa is a MS student working on this project for her thesis. She has been partially supported by this grant and partially supported by University funds. She is working on creating a new experimental setup for measuring friction response of various materials under typical process conditions.

Name: Kremer, James 
Worked for more than 160 Hours: Yes

Contribution to Project:

James is a new graduate student who just started on the project. He is working on finite element modeling of the forming process.

Name: Fetfatsidis, Konstantine

Worked for more than 160 Hours: Yes

Contribution to Project:

Graduate Research Assistant

Name: Morris, Corey

Worked for more than 160 Hours: Yes

Contribution to Project:

Graduate Research Assistant

\section{Undergraduate Student}

Technician, Programmer

Other Participant

Research Experience for Undergraduates

Name: Winchester, David

Worked for more than 160 Hours: Yes

Contribution to Project:

Undergradte Research Assistant

Years of schooling completed: Sophomore

Home Institution: Same as Research Site

Home Institution if Other:

Home Institution Highest Degree Granted(in fields supported by NSF): Doctoral Degree

Fiscal year(s) REU Participant supported: 2009

REU Funding: REU supplement

Name: Langworthy, Joanna

Worked for more than 160 Hours: Yes

Contribution to Project:

Performed experimental bending tests of fabrics and analyses of the experimental data to derive nonlinear moment curvature relationships for fabric bending to be added to the froming simulayion moldel.

Years of schooling completed: Junior

Home Institution: Same as Research Site

Home Institution if Other:

Home Institution Highest Degree Granted(in fields supported by NSF): Doctoral Degree

Fiscal year(s) REU Participant supported: 20102009

REU Funding: REU supplement

Name: Sargent, Joseph

Worked for more than 160 Hours: Yes

Contribution to Project:

Analyzed international benchmark data and assisted in the design of the next round of forming simulations to be completed by the international participants.

Years of schooling completed: Junior

Home Institution: $\quad$ Same as Research Site 


\section{Home Institution if Other: \\ Home Institution Highest Degree Granted(in fields supported by NSF): Doctoral Degree \\ Fiscal year(s) REU Participant supported: 20102009 \\ REU Funding: REU supplement}

\section{Organizational Partners}

\section{DOE -- Department of Energy}

This project is part of a jointly-funded effort by NSF and DOE on polymer composite manufacturing. The group of university researchers, DOE researchers (e.g., PNNL, ORNL), and automotive industry representatives meets annually to discuss critical needs, research directions, and opportunities for collaboration.

\section{Other Collaborators or Contacts}

1. We are part of an international benchmarking group that meets each year at the European Forming Conference (ESAFORM). This group has conducted experimental and modeling benchmark studies on materials characterization methods for fabrics (e.g., shear frame testing) and on simulation of fabric forming.

2. We have strong collaborations with several researchers in the US who are studying related issues, including but not limited to:

Prof Jian Cao, Northwestern University

Prof Dan Walczyk, RPI

Dr. Patrick Blanchard, Ford Motor Co.

Dr. Elizabeth Berger, General Motors

MAG-Industrial Automated Systems

\section{Activities and Findings}

\section{Research and Education Activities:}

Building on our past research, the primary activities for this project have focused on (1) Incorporation of material models for capturing the complex mechanical behavior of the into the ABAQUS and LS-DYNA finite codes using the explicit solver methods (2) Final adjustments to the apparatus for measuring friction between the metal tool and the woven-fabric composite and inter-layer shear, (3) Experimental investigation of the friction between the metal tool and the woven-fabric composite and faberic to fabric using load and displacement control methods and (4) inclusion of fabric bending stiffness.

Major education activities include poster presentations by James Kremer, Corey Morris and Konstantine Fetfatsidis at the UMass-Lowell student research conference. In addition, weekly briefs and bi-monthly presentations are made by all of the students in lab meetings. The finite elemnt models have been integrated into the PI's Introduction to Finite Elements course which is taken by senior undergraduate sttudents and graduate students.

\section{Findings: (See PDF version submitted by PI at the end of the report)}

Major research findings include:

1. Yarn and fiber parameters were investigated and their ability to model the compaction behavior of commingled fibers has been demonstrated by their influence on the resulting shear load-deformation curve.

2. A friction test apparatus was designed with respect to the processing parameters used during the thermostamping process of woven-fabric composites. The apparatus was used to conduct a parametric study which showed the tool temperature appeared to follow the relationship governed by polymer friction, where the polymer becomes tacky before reaching its melting point.

3. Stribeck theory was applied to the results obtained with the new apparatus to relate the static and dynamic coefficients of friction to the corresponding Hersey numbers. Past research assumed a constant film thickness for the calculation of the Hersey number. A new approach 
was developed in the last year that uses the experimental data to calculate the film thickness, thereby removing the need to assume a film thickness and giving a more accurate calculation of the Hersey number for use in the simulation.

4. The force-control application method allowed the dynamic coefficient of friction response of the fabric to be measured. Increasing normal force and decreasing velocity minimizes the dynamic coefficient of friction for the test parameters investigated. It had been stated in a previous report that the dynamic coefficient of friction was not converging to a steady state. After making some minor changes to the design of the 3rd-generation test apparatus, the data did show that a steady-state response does exist. Tilting of the load plates were comprising the data being collected.

5. Correlations have been made between the resulting stiffness as determined from the forming simulation and actual parts. Modal analyses completed using numerical and experimental methods have shown good correlation for the first two modes?both in mode shape and frequency. There was some divergence between the numerical and experimental data for higher modes. Additional research of flat plates was completed to develop a fundamental understanding of the reasons for the divergence. Variations in plate thickness were the root cause. Steps are now being taken to address these issues in the modeling.

6. The use of a variable friction model is critical to calculating the correct friction when doing a forming simulation. Under- or over-predicting the friction can give misleading results thereby compromising the rate at which a part can be formed without admitting wrinkling or tearing. Such is the case if a constant friction value is assumed. By having the simulation tool be able to calculate the actual friction sate as a function of pressure and speed, the forming rate may be increased fom that concluded from using a constant coefficient.

\&. The importance of fabric bending was explored.

On the education side, the MS and REU students involved in this project have gained a greater understanding of what research is and have improved their engineering and communication skills.

\section{Training and Development:}

1. Lu Liu, the doctoral student/postdoc, had already had four years of research experience, but gained some additional experience in teaching and supervising less experienced students.

2. Lisa Gamade, an MS student, gained significant experience in design of a novel experimental apparatus, including working with pneumatic loading systems, computer control, mechanical displacement mechanisms, and heating elements and controls. These experiences led to her being hired by Boeing in its Mesa, AZ location following her Summer 2007 graduation.

3. James Kremer, an MS student, gained knowledge in how to implement user-supplied material models in ABAQUS/Standard abd ABAQUS/Explicit to address an orthotopic material with highly nonlinear deformation.

4. Kostantine Fetfatsidis, an MS student, gained experience in the refining of the design of the friction test apparatus, collection and analysis of experimental data, implementation of user-supplied material and friction models in ABAQUS and LS-DYNA.

5. Corey Morris, an MS student, gained experience in the implementation of user-supplied material and friction models in LS-DYNA and the applicability of the various 1D and 2D ements in LS-DYNA for completing forming simulations.

6. Joanna Langworthy, REU student, gained the experience of conduction modal and fabric bending experiments and the analyses of the resulting data.

7. David Winchester, REU student, gained experience in the completion of material characterization experiments and the calculation of material parameters from these experimental data, how to convert CAD geometry to a form that can be used to develop a finite element mesh, how to use the finite element method to run forming simulations and modal analyses.

8. Joseph Sargent, REU student, learned how to take data from international researchers and see similarities and differences and to use this information to design a plan for the various research groups to compete a set of simulations for a uniform comparison of the capabilities of the respective modeling tools for forming of the woven composites. 
9. David Jauffres, post doc, gained some additional experience in teaching and supervising less experienced students and extended his expertise in polymer material science to using finite element methods for the forming polymer-matrix composites.

\section{Outreach Activities:}

Tours of the lab are regularly provided to visitors (typically from industry).

Work with the UMass-Lowell College of Engineeing Design Camp (Grade 3-11 students) to give hands-on demonstrations of composite braiding and resin application to make a polymer-matrix composite tube.

\section{Journal Publications}

Jauffres, David ; Sherwood, James ; Morris, Corey ; Chen, Julie, "Discrete Mesoscopic Modeling for Woven Fabric Reinforcement Forming Simulation", International Journal of Material Forming, p. 17, vol. 2 Suppl, (2009). Published,

Cao, J; Akkerman, R; Boisse, P; Chen, J; Cheng, HS; de Graaf, EF; Gorczyca, JL; Harrison, P; Hivet, G; Launay, J; Lee, W; Liu, L; Lomov, SV; Long, A; de Luycker, E; Morestin, F; Padvoiskis, J; Peng, XQ; Sherwood, J; Stoilova, T; Tao, XM; Verpoest, I; Will, "Characterization of mechanical behavior of woven fabrics: Experimental methods and benchmark results", COMPOSITES PART A-APPLIED SCIENCE AND MANUFACTURING, p. 1037, vol. 39, (2008). Published, 10.1016/j.compositesa.2008.02.01

Fetfatsidis K.A., Gamache L., Sherwood J.A., Jauffr??s D. and Chen J., "Design of an Apparatus for Measuring Tool/Fabric and Fabric/Fabric Friction of Woven-Fabric Composites During the Thermostamping Process", International Journal of Material Forming, p. , vol. , (2010).

Submitted,

\section{Books or Other One-time Publications}

Gamache, L.M., Sherwood, J.A., Chen,J., and Cao, J., "Characterization of the Fabric/Tool and Fabric/Fabric Friction during the Thermostamping Process", (2007). Conference Proceeding, Published

Collection: Proceedings of the 10th ESAForm Conference on Material Forming, Zaragosa, Spain

Bibliography: Gamache, L.M., Sherwood, J.A., Chen,J., and Cao, J., 2007, "Characterization of the Fabric/Tool and Fabric/Fabric Friction during the Thermostamping Process", Proceedings of the 10

Lisa M. Gamache, "The Design and Validation of a Friction Test Apparatus based on the Thermostamping Process of Woven-Fabric Composites", (2007). Thesis, Published

Bibliography: Gamache, L.M., 2007, The Design and Validation of a Friction Test Apparatus based on the Thermostamping Process of Woven-Fabric Composites, MS Thesis, University of Massachusetts L

K.A. Fetfatsidis, J.A. Sherwood, J. Chen, and D. Jauffres, "Characterization of the Fabric/Tool and Fabric/Fabric Friction during the Thermostamping Process", (2009). Conference Paper, Published

Bibliography: Proceedings of the 12th International Conference on Material Forming Twente, Netherlands

D. Jauffres, C.D. Morris, J.A. Sherwood, J. Chen, "Simulation of the Thermostamping of Woven Composites: Determination of the Tensile and In-plane Shearing Behaviors", (2009). Conference Paper, Published

Bibliography: Proceedings of the 12th International ESAFORM Conference

D. Jauffres, C.D. Morris, J.A. Sherwood, J. Chen, "Simulation of the Thermostamping of Woven Composites: Mesoscopic Modelling using Explicit FEA Codes", (2009). Book, Published

Bibliography: Proceedings of the 12th International ESAFORM Conference

Konstantine Fetfatsidis, "Characterization of the Tool/Fabric and Fabric/Fabric Friction for Woven Fabrics: Static and Dynamic", (2009).

Thesis, Published

Bibliography: UMass-Lowell 
Sargent J., Chen J., Sherwood J., Cao J., Boisse P., Willem A., Vanclooster K., Lomov S.V., Khan M., Mabrouki T., Fetfatsidis K., Jauffr?s D., "Benchmark Study of Finite Element Models for Simulating the Thermostamping of Woven-Fabric Reinforced Composites", (2010).

Conference, Published

Bibliography: Proceedings of the 13th International ESAFORM Conference. Brescia, Italy

Sherwood J., Fetfatsidis K., Winchester D., Jauffr?s D., Avitabile P., Chen J., "Using Modal Analysis to Investigate the Validity of Finite Element Models for Simulating the Thermostamping of Woven-Fabric Reinforced Composites", (2010). Conference, Published

Bibliography: Proceedings of the 13th International ESAFORM Conference. Brescia, Italy

\section{Web/Internet Site}

\section{URL(s):}

http://m-5.eng.uml.edu/acmtrl/

Description:

\section{Other Specific Products}

\section{Product Type: \\ Software (or netware) \\ Product Description:}

The software is a set of subroutines that can be integrated with the ABAQUS and LS-DYNA commercially available finite element packages for describing the mechanical behavior of woven fabrics and the fabric/fabric and tool/fabric frictions during the forming of woven-fabric composites.

\section{Sharing Information:}

The software has been shared with indutrial partners through doing collaborative research.

\section{Contributions within Discipline:}

\section{Contributions}

Because of the unique heterogeneous, non-continuum nature of fiber assemblies (e.g., yarns and fabrics), standard models originally derived for bulk metals and polymers are often not applicable. Our findings in the analytical modeling of yarn friction and compaction will help to improve models of the material response in forming processes and subsequently the in-service performance of the formed part.

The novel friction measurement instrument is now complete and will provide valuable experimental data for providing insight into governing mechanisms and for validation of models.

The implementation of the material models into the finite element codes ABAQUS and LS-DYNA allows the simulation of the manufacturing process and subsequent ability to analyze the in-service structural performance. ABAQUS and LS-DYNA are commercially available codes that are used extensively by engineers in the automotive industry. Thus, it should be relatively seamless to transfer this technology from academia to application in industry.

As a result of the successes of this research, the team has applied the design tool to the forming of a tub (car trunk) and floor pan for a USCAR project. The design suceessfully showed what layups are acceptable for making these car parts from woven-fabric composites and the shape of the blank fabrics to be used in the manufacturing process. GM and Ford are working cooperatively to integrate the design tool into their product development process.

\section{Contributions to Other Disciplines:}

\section{Contributions to Human Resource Development:}

This project has involved three faculty (two female) and four graduate students (one female) and two post docs. The graduate students have benefited from seeing how their engineering research can help solve real-world problems for the automotive industry. An REU students was 
added in Year 3 and two more in Year 4.

The post doc, one graduate student and the REU student had the opportunity to go to Ford to observe and conduct stamping operations for a sample complex geometry. Thus, these three were able to experience the design of a manufacturing process using the simualtion tool and see the manufacturing process of the part.

The knowledge gained through this project gave Lisa Gamache (female MS student) extensive experience in machine design, controls, data acquisition, material testing and analysis which in turn led to her being hired by Boeing for their structural group in Mesa, AZ.

\section{Contributions to Resources for Research and Education:}

Novel testing apparatus for the characterization of friction

Simulation tool is being integrated by Ford and GM into their composite products development process.

\section{Contributions Beyond Science and Engineering:}

Modeling of the in-service performance of thermoplastic-matrix woven-fabric reinforced structural composites ? e.g., damage tolerance, crashworthiness, vibration? is currently inadequate for parts of any geometric complexity because of the inability of existing performance models to capture the true deformed material properties. As a result, lightweight, more energy-efficient, composite materials are utilized inefficiently or not at all.

Alternatively, extensive experimental trial and error or design of experiments must be conducted to develop a satisfactory product. Such approaches reduce the competitiveness of US industry in terms of more innovative designs and time-to-market.

The key to addressing this barrier is to provide a direct link between part geometry, material selection, process conditions, process-induced local properties, and part performance, allowing informed feedback to the design process.

\section{Conference Proceedings}

Pingle, PS;Gorbatikh, L;Sherwood, JA, Analysis of multiple rigid-line inclusions for application to bio-materials, "NOV 11-15, 2007", PROCEEDINGS OF THE ASME INTERNATIONAL MECHANICAL ENGINEERING CONGRESS AND EXPOSITION 2007, VOL 10, PTS A AND B, : 801-807 2008

Gamache, LM;Sherwood, JA;Chen, J;Cao, J, Characterization of the Fabric/Tool and Fabric/Fabric friction during the thermostamping process, "APR 18-20, 2007", 10th ESAFORM Conference on Material Forming, Pts A and B, 907: 1068-1073 Part A-B 2007

Lee, W;Cao, H;Chen, J;Sherwood, J, Numerical analysis on double dome stretching tests of woven composites, "APR 18-20, 2007", 10th ESAFORM Conference on Material Forming, Pts A and B, 907: 1052-1057 Part A-B 2007

Contributions: To Any Other Disciplines

\section{Categories for which nothing is reported:}


NSF GRANT \# DMII-0522923

NSF PROGRAM NAME: MPM

Annual Report for Year 4:

Linking Process-Induced Properties to Thermoplastic-Matrix Woven-Fabric Composites Performance

\author{
James Sherwood \\ Julie Chen \\ Larissa Gorbatikh \\ University of Massachusetts Lowell
}

\begin{abstract}
To model the in-service mechanical behavior of thermoplastic-matrix woven-fabric reinforced structural composites, the mechanical properties resulting from the manufacturing process need to be known. This report will discuss the research tasks that have been completed through the fourth year of a research effort to develop a design tool which links the process-induced mechanical properties of thermoplastic-matrix woven-fabric composites to their in-service performance. To study the friction relationship between the forming tool and the deformed fabric geometry, the design and application of a novel test method is presented. Modal analysis is used to examine the correlation between the structural stiffnesses of the forming simulation to that of a complex three-dimensional formed geometry.
\end{abstract}

\title{
1.0 Introduction
}

Modeling of the in-service performance of thermoplastic-matrix woven-fabric-reinforced structural composites e.g., damage tolerance, crashworthiness, vibration - is currently inadequate for parts of any geometric complexity because of the inability of existing performance models to capture the true deformed material properties. As a result, lightweight composite materials are utilized inefficiently or not at all; alternatively, extensive experimental trial and error or design of experiments must be conducted to develop a satisfactory product. The key to addressing this barrier is to provide a direct link among part geometry, material selection, process conditions, process-induced local properties, and part performance, allowing informed feedback to the design process. Fiber-reinforced thermoplastic composites have a variety of applications including structural components in the automotive, aerospace, marine, infrastructure and recreation industries. The main advantage of these composites over metals is their high specific strength. They also have other beneficial properties including low thermal expansion and good corrosion resistance when compared to metals. Woven fabrics offer many other advantages when compared to metals in terms of deformation capabilities, including dimensional stability, good conformability, and deep-draw shapability. Compared to nonwoven-fabric composites, the woven-fabric composites provide more balanced properties, higher impact resistance, easier handling and lower fabrication cost, particularly for parts with complex shapes.

The objective of this research is to utilize an integrated analytical, experimental, and numerical effort to attain a fundamental understanding of two governing physical phenomena - interlayer (fabric-fabric) friction and interconnected through-thickness and in-plane compaction. The former is important in multiple-layer and multiplestep forming, both needed for industrial processes. The latter is a critical factor affecting the final part thickness, void content, fiber orientation, and fiber distribution - all are important for structural stiffness and damage tolerance. To validate and demonstrate the capabilities of the integrated structure-process-performance design tool, the model will be used to identify critical process and material parameters for one particular performance condition - vibration.

A combined inter-tow friction model, developed in a previous NSF-funded research program (DMII-0331267), based on the equilibrium equations of the unit cell of a balanced plain-weave glass/polypropylene woven fabric is extended to account for fiber compaction. To accomplish this extension of the model, fiber-compaction experiments were completed and the results are reported.

The design and use of a $3^{\text {rd }}$-generation friction-testing apparatus is presented. Previous research efforts used a constant-displacement control device to load the undeformed fabrics during the friction testing. While the information learned in these tests was valuable in developing a fundamental understanding of the tool-fabric behavior, the $3^{\text {rd }}$-generation friction-testing apparatus is a load-control device and yields a very complete fundamental understanding of the friction mechanisms by being able to study inter-layer friction between fabrics and tool/fabric friction on undeformed and deformed fabrics and to capture the static and dynamic friction behaviors of a woven-fabric system. Results are reported for two material systems - one has a thermoplastic matrix and the other has a thermoset matrix. 
The methodology has been implemented in the commercially available finite element programs ABAQUS and LSDYNA. The elements for the fabric models are inherent to these finite element programs, so no special coding of elements is required. The material and friction behaviors are incorporated in the finite element modeling by userdefined subroutines with simple input cards for specifying material and friction properties. Thus, the design tool is packaged in a form that makes the transition to industry relatively seamless and easily used by composite design engineers in industry.

The method has already been shown to be attractive to industry. During 2008-09, the UMass-Lowell research team collaborated with industrial colleagues at Ford Research Lab and General Motors to do forming simulations of a rear tub (trunk) and floorpan for the USCAR program with DOE funding. MAG-Industrial Automated Systems has expressed interest in collaborating with UMass-Lowell to use the simulation method to explore the forming of automotive parts using prepreg unidirectional tapes and woven-fabric composites with embedded optical fibers.

\subsection{The Thermostamping Manufacturing Process}

Thermostamping is a manufacturing process that can produce quality composite parts with processing costs and time margins that are comparable to the fabrication of the currently stamped metal parts. The thermostamping process deforms a flat sheet of machine-woven continuous fibers (glass and/or carbon) commingled with thermoplastic fibers into a particular geometry using a pair of dies.

The thermostamping process (Fig. 1) starts with the alignment of a sheet of fabric in a rigid steel frame. Multiple layers of fabric are stamped into one part to achieve the desired part thickness and mechanical properties. For a thermoplastic-matrix woven fabric, the frame and fabric are placed in an oven and are soaked until the fabric reaches a desired temperature for the thermoplastic polypropylene fibers, which are commingled with fiberglass fibers, to melt. The melting of the thermoplastic fibers infuses the fiberglass fibers with the resin. The frame is then aligned with a punch and die, and pressure is applied around the circumference of the punch area by binder plates. The application of force by the binder plate provides the in-plane forces that are necessary to reduce wrinkling of the fabric as the woven material is drawn into the die by the punch. The finished piece assumes the geometry of the die and punch and hardens into a firm part after the thermoplastic has cooled. For the thermoset material, the woven fabric is compounded with a pliable thermoset resin. The frame holding the woven fabric is stamped into shape using the punch and die and then held at elevated temperature to cure (harden) the thermoset resin matrix. A hemisphere formed from a commingled glass-polypropylene fabric using this thermostamping process is shown in Fig. 2. The primary mode of deformation for the fabric is rotation (shearing) of the yarns, also known as 'trellis deformation'.

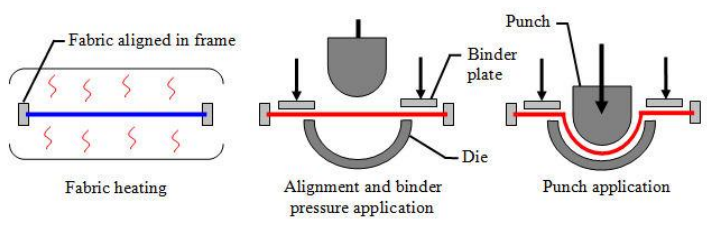

Fig. 1 Schematic example of the thermostamping process. 




Fig. 2 Formed hemisphere using the thermostamping process.

The processing parameters associated with the thermostamping operation can have a significant effect on part quality. Fig. 3 shows the resulting part quality in a section under the binder plate from the stamping process of a commingled glass-polypropylene woven-fabric as studied by Wilks [1]. The resulting part quality is shown for cases of different pressure-and-temperature combinations. For this test, the pressure applied by the binder plate was controlled by springs and the binder plate was either unheated or heated during the process. With the binder plate unheated, fiber separation and tow breakage occurred sooner than when the binder plate was heated as pressure was increased (Fig. 3: d, f). The presence of fiber wrinkling was reduced as pressure increased (Fig. 3: e). Thus, it can be observed that part quality is a function of temperature and applied force, and in turn friction between the fabric and the tool.

The mechanical behavior of the woven fabric during the forming process is dominated by the evolution of the inplane shear stiffness as the yarns rotate and the uncrimping of the yarns due to in-plane tensile forces. By using a series of simple tests to characterize the mechanical behavior of a woven fabric, material parameters can be found. As an alternative to experimental characterization, there has been some limited success to develop purely analytical models of woven fabrics to estimate the mechanical behavior of a woven fabric. These analytically and/or experimentally determined material properties can be used in a finite element program to simulate the manufacturing process of a composite structure.

The inextensible characteristic of the yarns (fiberglass and/or carbon) requires draw-in and shearing of the fabric to occur for the material to conform to the tool geometry in forming the part. During fabric draw-in, the fabric slides under the metal binder plate and over the surfaces of the punch and die. For multilayer parts, there is possibly going to be some sliding between adjacent layers of fabric. Considering the interactions that take place between the fabric and tool and between adjacent layers of fabric during the manufacturing process and the effect of the process parameters, e.g. pressure, temperature and rate, on part quality, it is clear that characterizing the friction between the tool and the fabric and the friction between adjacent layers of fabric is critical to modeling the manufacturing process accurately.

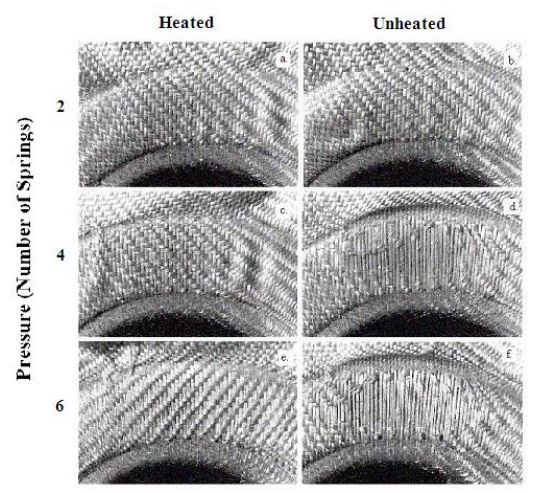

Fig. 3 Effect of temperature and pressure on part quality during the thermostamping process [1]. 


\subsection{Analytical Modeling of the Shear Behavior of Woven-Fabric Composites}

Trellis shear, as shown in Fig. 4, is the main deformation mode during the stamping process of woven-fabric composites [2]. Thus, the formability of woven-fabric composites is primarily a function of the shear properties of the woven fabric. Therefore, analytical models to obtain the shear properties of woven-fabric composites would be very useful to characterize the mechanical behavior of an existing fabric without doing time-consuming experiments or for characterizing a yet to made fabric for which experimentation is not an option.
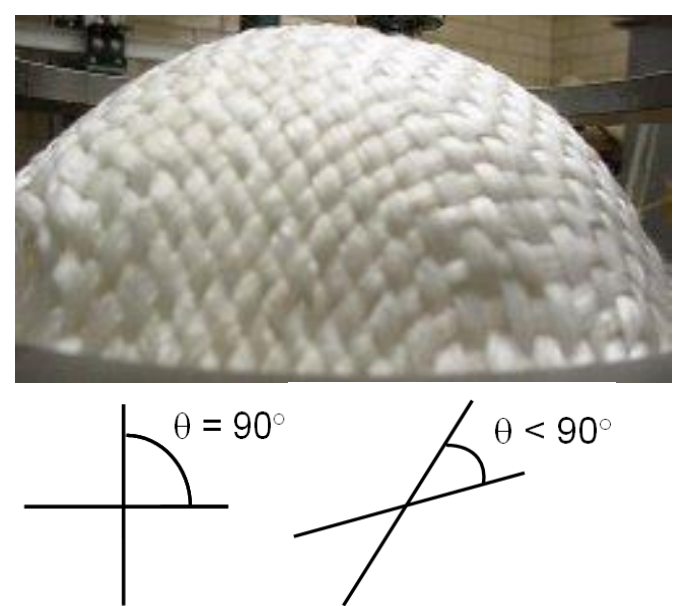

Fig. 4 Trellis deformation of a stamped hemisphere.

An analytical unit-cell model, which includes (1) the key shear-deformation-resistant mechanisms, (2) the friction between the warp and weft yarns at every crossover, and (3) the lateral compaction between adjacent yarns, was developed by Liu et al. $[3,4]$ to predict the shear properties of woven fabrics for the thermostamping process. In the model, some yarn parameters such as the yarn-to-yarn coefficient of friction $\mu$, the fiber contact frequency ratio $\beta$, and the ideal maximum fiber volume fraction $V_{a}$, were chosen to develop an empirical model to fit the experimental data. Validation of those parameters is necessary to complete the unit-cell model.

The maximum yarn-fiber volume fraction $V_{a}$ is an ideal limit to which fibers in a yarn can be compacted. As the yarn-fiber volume fraction approaches this maximum value, the stiffness in the transverse direction increases dramatically to approach the stiffness of the solid fiber material. According to the packing theory of uniform sizes of fibers, square packing corresponds to $V_{a}$ of $\pi / 4$ (or 0.785), and hexagonal packing results in a value of $\sqrt{3} \pi / 6$ (or 0.907). Because the diameters of the polypropylene and glass fibers are different in the commingled polypropylene/glass yarn, the square packing theory is modified for the commingled fibers, and the obtained $V_{a}$ equals 0.813 [5].

The compaction was studied using plane-strain compression tests, where $50-\mathrm{mm}$ long fiber bundles were placed in a channel fixture as shown in Fig. 5. A compressive force was applied perpendicular to the axial direction of the fibers ( $Z$ direction) using an Instron machine at a rate of $0.042 \mathrm{~m} / \mathrm{sec}$. Load and extension data were logged so that stress and strain in the $Z$ direction could be calculated. Because the transverse compliance can also be calculated in the fiber bundle model as a function of $\beta$, regression of the experimental data will give the mean value of $\beta$. In Fig. 6 , the predicted and experimental values of the yarn transverse compliance $S_{22}$ are compared for various values of $\beta$. The experimental results were obtained from the plane-strain compression tests for five layers of yarns as described in [6]. From Fig. 6, it can be seen that the values of $\beta$ are in the range from 150 to 175 for the commingled polypropylene and glass yarns explored in this research.

To investigate the sensitivity of the model to the yarn input parameters such as the maximum fiber volume fraction $V_{a}$, the fiber contact ratio $\beta$ and the yarn-to-yarn coefficient of friction $\mu$, a parametric study was conducted. To separate the individual contributions from friction and lateral compaction, intermediate models were studied separately, i.e., the lateral compaction resistant moment was set to be zero to study the coefficient of friction, while in the study of $\beta$ and $V_{a}$, the friction resistant moment was set to be zero in the equilibrium equation. 
Fig. 7 shows the results of the simulation of $\beta$ varying from 150 to 175 . In the figure, it can be seen that as the value of $\beta$ decreases, the shear load increases. The loads increase because at relatively low values of $\beta$, the yarn stiffness in the transverse direction increases due to increased fiber bending. As shown in Fig. 8, decreasing the value of $V_{a}$ has a similar effect because, with relatively low values of $V_{a}$, yarn stiffness in the transverse direction increases due to decreased space for compaction. Thus, with the main parameters all validated, the developed analytical model can be used to predict the shear properties of woven-fabric composites and reduce or eliminate the need for curve fitting of fabric-level experimental data.
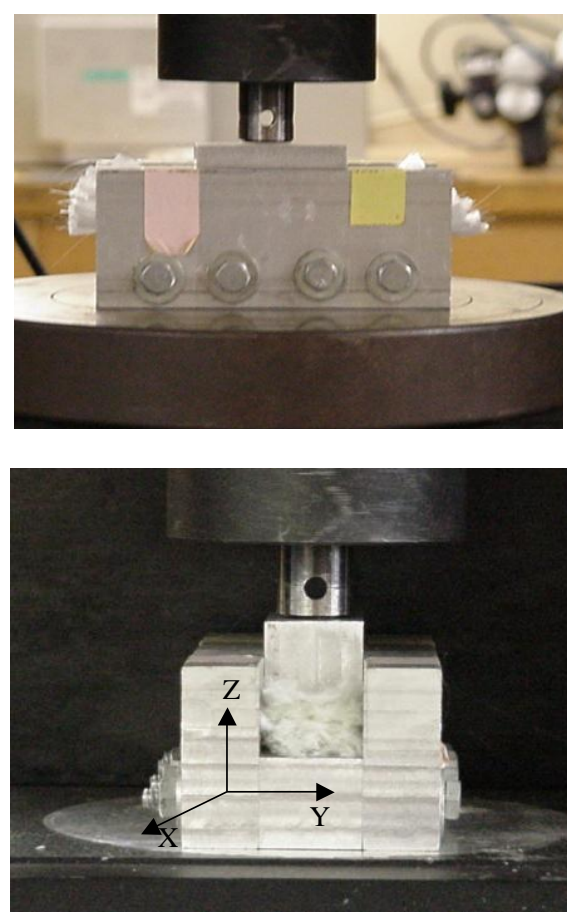

Fig. 5 Plane-strain compression test fixture [5].

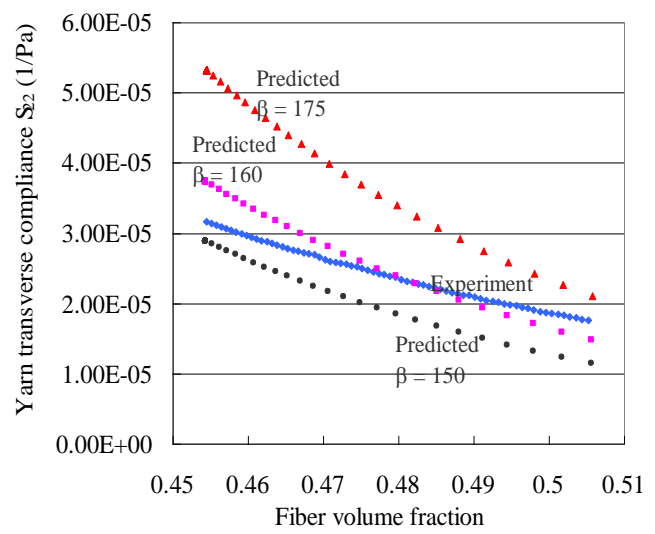

Fig. 6 Comparison of the values of $S_{22}$ obtained from the plane-strain compression tests and the fiber bundle modeling [6]. 


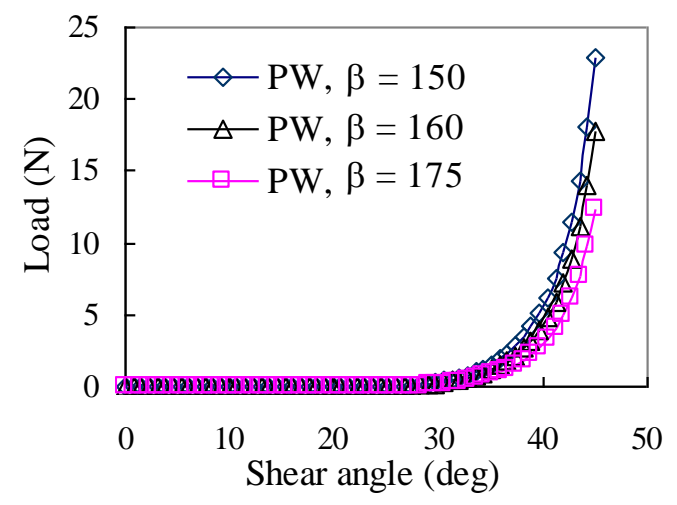

Fig. 7 Simulation of the fiber contact frequency ratio.

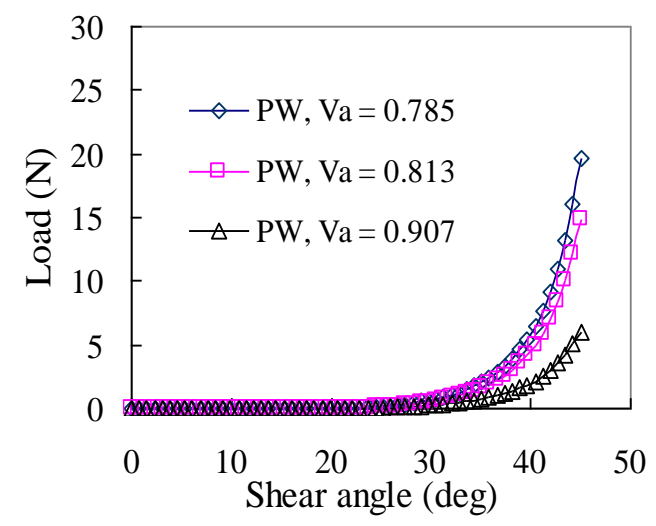

Fig. 8 Simulation of the maximum fiber volume fraction.

\subsection{Friction Behavior for Woven Fabrics}

Past NSF-sponsored research led to advances in a user-defined friction subroutine for use in finite-element applications. The Stribeck curve (Fig. 9 [7]) and the Hersey number, H, (Eq. 1) were used to find the friction coefficient for the ranges of velocities and normal forces studied.

$$
H=\frac{\eta \cdot U}{N}
$$

where $\eta$ is the viscosity, $U$ is the fabric velocity and $N$ is the normal force.

The results of parametric studies conducted by Gorczyca [8,9] showed that the velocity of the fabric, tool temperature, and applied normal force had the greatest effects on the friction coefficient. From these results, she developed a model that related the friction coefficient $\mu$ to the Hersey number,

$$
\mu=(6.1191 \cdot H+0.2718)-\mu_{\theta}
$$

where $\mu_{\theta}$ is a scaling term included for the effects of the tool temperature. Because the method of normal force application used in her research was displacement controlled, her results were limited to measuring only the static coefficient of friction $[8,10,11]$.

It was found that during a typical friction test the normal force applied to the fabric surface decreased, as shown in Fig. 10 where the initial applied load was approximately $1500 \mathrm{~N}$ and dropped by $\sim 400 \mathrm{~N}$ over the duration of the 
test. The mechanisms behind this decrease in normal force are fabric nesting and tow compaction. The fabric tows adjust their position as the adjacent layers of fabric nest, tow undulation decreases and fibers compact due to the combination of heat and pressure. Any one or more of these mechanisms causes a slight change in the displacement necessary to maintain the targeted normal force.

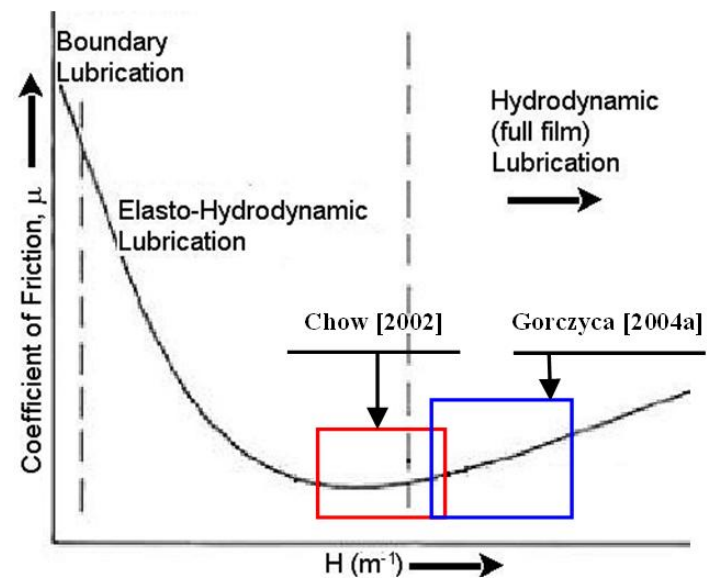

Fig. 9 The Stribeck Curve and areas of interest pertaining to current research. Adapted from [7].

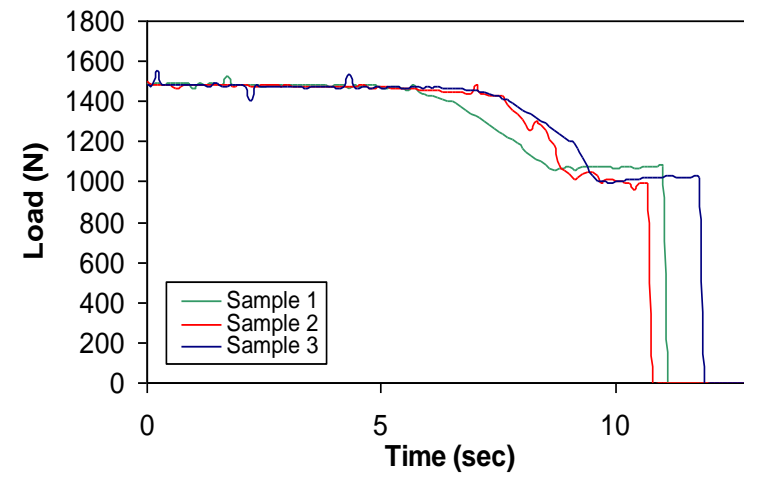

Fig. 10 Normal force decrease over time for displacement controlled test of commingled glass-polypropylene plain-weave fabric.

Due to the drop in normal force, only the static coefficient of friction, which is the value obtained at the first instant of fabric movement, could be reliably quantified by Gorczyca using the $2^{\text {nd }}$-generation displacement-control friction test apparatus. Being able to capture the dynamic coefficient of friction would give a friction model that accurately represents the frictional behavior over the entire duration of the thermostamping process where the friction is both static and dynamic.

To gain the ability to determine the dynamic coefficient of friction, a load-controlled application mechanism was designed and built as one component of the current research [12]. The force-controlled mechanism consists of a pneumatic air-spring actuator and a closed-loop control algorithm to continuously monitor and regulate the magnitude of force applied to the fabric throughout the friction test. The temperature of the tool and the pullout speed can be prescribed over a range of values that are observed to be present in the thermostamping process.

For the stamping of a hemisphere with a plain-weave fabric (as shown in Figs. 1 and 2), the tows are initially oriented $\sim 90^{\circ}$ with respect to one another, and this angle decreases as the fabric conforms to the dual-curvature surface of the hemisphere as shown in Fig. 4. 


\subsection{Friction-Testing Apparatus}

The $3^{\text {rd }}$-generation friction-testing apparatus is a stand-alone test machine. The limiting factor for the previous $1^{\text {st }}$ and $2^{\text {nd }}$-generation test apparatuses was their dependence on the Instron testing machine. By integrating all fabric movement into one test apparatus, the travel time from the oven to the press can be reduced from $\sim 20$ seconds to be less than 3 seconds, thereby reducing the influence of any cooling effects as the fabric is transferred from the oven to the press. Fig. 11 shows a schematic of the overall $3^{\text {rd }}$-generation friction-testing system, and Fig. 12 is a photograph of the press portion of the system.



Fig. 11 Schematic representation of the $3^{\text {rd }}$-generation friction test apparatus.

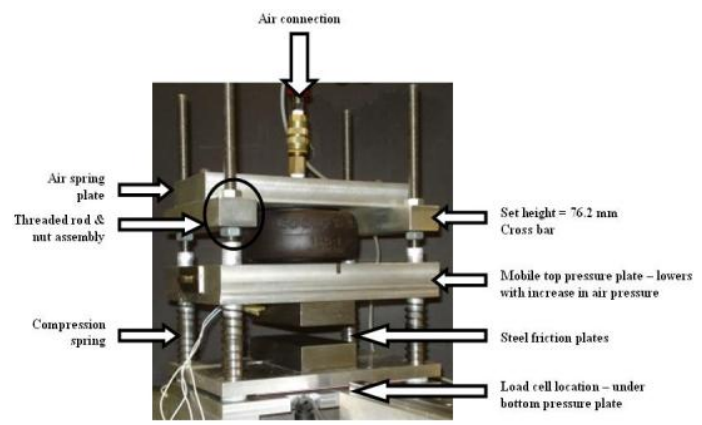

Fig. 12 The friction press.

\subsection{Friction Experiments}

The $3^{\text {rd }}$-generation load-control friction test apparatus [13] was used to capture the static and dynamic coefficients of friction. The objective of the friction-test apparatus was to accurately simulate the combinations of pressure, temperature and speed observed in the actual thermostamping process. In a friction test, the fabric is clamped in a holder (Figure 13(a)), heated in an infrared oven and subsequently shuttled to the press (similar to what is shown in Fig. 1 for the thermostamping process), which features two heated platens. A DC motor drives a rack and pinion to pull the sample through the press. The transfer from the oven to the press is done manually, and the fabric sample typically sits pressurized between the platens for about one second before the motor begins to pull. Experiments have shown that this one-second time is sufficient to keep the thermoplastic melted. 


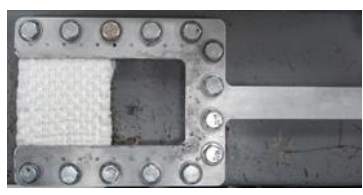

(a)

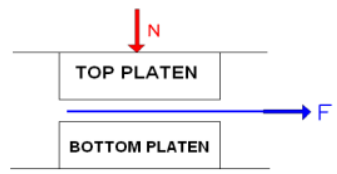

(b)

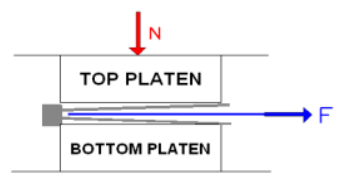

(c)

Fig. 13 (a) Fabric holder and schematics of the friction test setups for

(b) tool/fabric and

(c) fabric/fabric interfaces

For the fabric/fabric friction characterization, one fabric layer is placed between two "outside" layers of fabric. The two outer layers are clamped together on one end, and the inner layer is pulled between these two outer layers. The fully compressed platens prohibit the clamp which holds the two outer layers from moving. The fabric holder which is used to pull the fabric through the press is shown in Fig. 13(a) and schematics of the friction test setup for the fabric/tool and fabric/fabric conditions are shown in Figs. 13(b) and (c), respectively.

The effective coefficient of friction is calculated by

$$
\mu_{e f f}=\frac{F}{2 N}
$$

where the normal force, $N$, is multiplied by a factor of two to account for the two contacting surfaces on either side of the fabric sample.

The test conditions were also based on simulating a range of thermostamping process conditions. Experiments were performed on a Twintex ${ }^{\circledR}$ balanced plain-weave fabric. All samples were cut to be $51 \times 76 \mathrm{~mm}^{2}$, and tests were performed in triplicate to account for any variation among fabric samples.

The coefficient of friction was plotted against the pullout displacement. These curves can result in an initial peak, followed by a drop and then a relatively flat response for the Twintex ${ }^{\circledR}$ woven fabric used in this research. The peak and "flat" portions of the curves correspond to the static and dynamic coefficients of friction, respectively. The magnitude of the peak is a function of the pulling speed and pressure. Fig. 14 shows typical coefficient of friction vs. displacement curves for three tests at a pressure of $110 \mathrm{kPa}$, pullout velocity of $16.7 \mathrm{~mm} / \mathrm{s}$, and tool temperature of $85^{\circ} \mathrm{C}$.

A constant fabric area remains between the platens for approximately $50 \mathrm{~mm}$ of displacement. A drop in the fabric temperature after the fabric is in contact with the platens explains why small variations in the pullout force in the displacement range associated with the dynamic coefficient of friction are observed. 


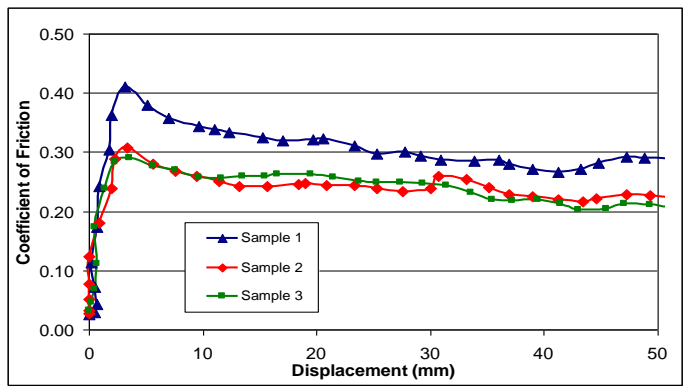

Fig. 14 Coefficient of friction as a function of pullout length for three tests at a pressure of $110 \mathrm{kPa}$, pullout velocity of $16.7 \mathrm{~mm} / \mathrm{s}$, and tool temperature of $85^{\circ} \mathrm{C}$.

\subsection{Friction Test Results}

Fig. 15 shows the static and dynamic coefficients of friction at the fabric/tool interface for the Twintex ${ }^{\circledR}$ balanced plain-weave fabric as a function of pullout velocity [14]. All tests were run with the baseline pressure of $387 \mathrm{kPa}$ and a tool temperature of $85^{\circ} \mathrm{C}$. The baseline values were determined from the typical processing conditions for the Twintex ${ }^{\circledR}$ fabric being considered in this research. In Stribeck theory, an increase in velocity causes an increase in the Hersey number. Similarly, an increasing velocity causes viscosity to increase, which also increases the Hersey number. In the hydrodynamic range of the Stribeck curve, an increasing Hersey number corresponds to an increasing coefficient of friction. Fig. 15 implies that the friction response is in the hydrodynamic behavior region of the Stribeck curve (Fig. 9) because the coefficient of friction increases with increasing velocity and likewise increasing Hersey number. Quantifying the variation of the friction coefficient as a function of velocity is important because during the forming process, the fabric does not necessarily move at a constant velocity along the tooling. In addition, as the fabric conforms to the shape of the die, the tows of the fabric are sheared and reoriented, affecting the rate at which the fabric slides over the surfaces that it is contacting.

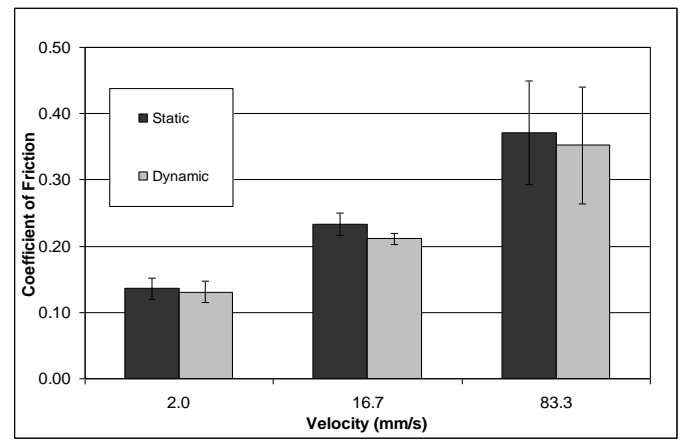

Fig. 15 Experimental results (fabric/tool) at different pullout velocities with the baseline pressure of $387 \mathrm{kPa}$ and the baseline tool temperature $85^{\circ} \mathrm{C}[14]$.

Fig. 16 shows the variation of the static and dynamic coefficients of friction with changes in normal force (or pressure). The pullout speed was the baseline value of $16.7 \mathrm{~mm} / \mathrm{s}$. In Eqn. (1), it can be seen that normal force is inversely related to the Hersey number. Therefore, the decrease in the friction coefficient with an increase in normal load shown in Fig. 16 also implies the friction behavior to be in the hydrodynamic-friction region of the Stribeck curve (Fig. 9). 


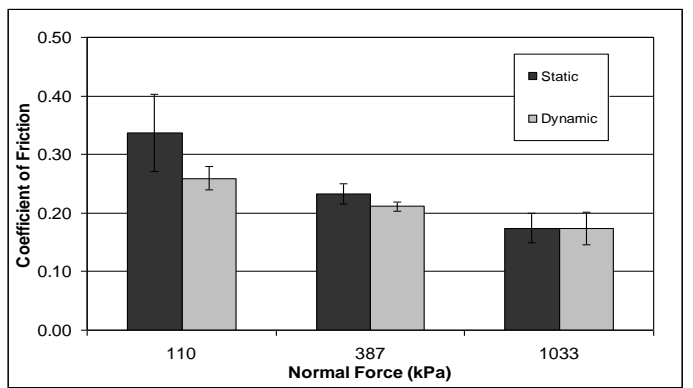

Fig. 16 Experimental results (fabric/tool) at different normal loads with the baseline pullout velocity of $16.7 \mathrm{~mm} / \mathrm{s}$ and the baseline tool temperature of $85^{\circ} \mathrm{C}[14]$.

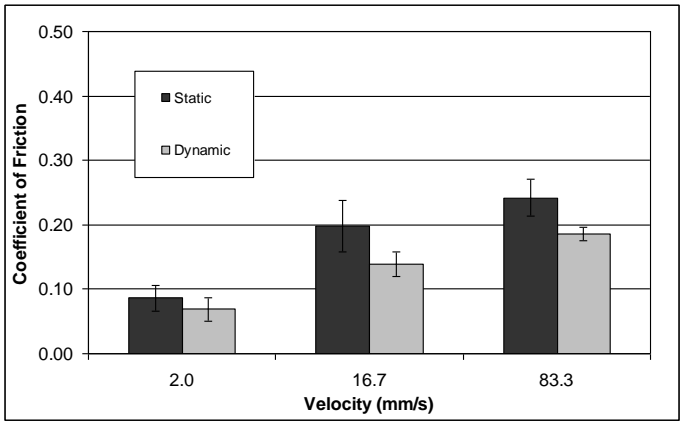

Fig. 17 Experimental results (fabric/fabric) at different pullout velocities with the baseline pressure of $387 \mathrm{kPa}$ and the baseline tool temperature of $85^{\circ} \mathrm{C}$ [14].

Fig. 17 shows the static and dynamic coefficients of friction for the balanced plain-weave fabric at the fabric/fabric interface as a function of pullout velocity. Similar to the fabric/tool interface, the coefficient of friction was observed to increase with increasing velocity at the fabric/fabric interface.

Fig. 18 displays the effect of normal force on the friction coefficient at the fabric/fabric interface. The decrease in the coefficient of friction with increasing normal force follows the same trend as the results from the fabric/tool interface. Similar trends for the coefficients of friction as a function of normal force and velocity were observed at both the fabric/tool and fabric/fabric interfaces, where only the magnitude of the friction coefficients differed. These trends support that the Stribeck curve can explain the observed friction behaviors.

Due to significant differences in friction coefficients under different processing conditions, any finite element simulation of the manufacturing process must be able to capture the coefficient of friction as a function of pressure, velocity and temperature. Such dependencies have been implemented in ABAQUS/Standard [8] ABAQUS/Explicit, and LS-DYNA (explicit version) $[15,16]$ through user-defined friction subroutines for finite element modeling of the thermostamping process.

Friction testing was completed to study the effect of fabric orienation and the state of shear. For tool/fabric and fabric/fabric frictions, the the experimental data implied that these paramters were essentially negligle on the resulting friction coefficients. 


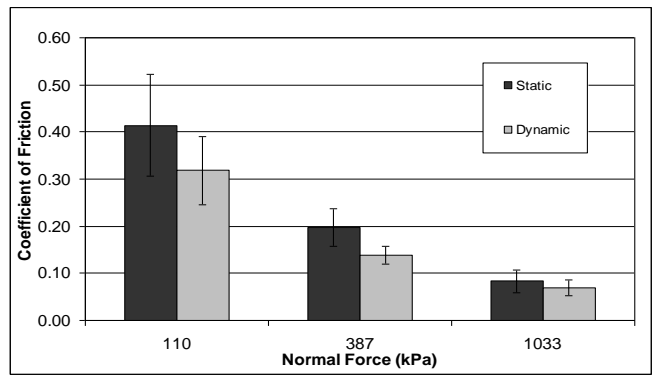

Fig. 18 Experimental results (fabric/fabric) at different normal loads with the baseline pullout velocity of $16.7 \mathrm{~mm} / \mathrm{s}$ [14].

\subsection{Characterization of the Mechanical Behavior of the Woven Fabrics using Experimental Methods}

Experimental data were used to derive the material parameters for the constitutive models used in the simulation of the mechanical behavior of the fabrics studied in the forming simulations of the current research. For the forming of composite parts using woven fabrics, these material parameters were needed to describe the nonlinear tensile behavior of the yarns and the nonlinear shear behavior of the fabric.

A yarn behaves differently when incorporated within a crimped fabric than alone because of the undulations of the yarns and the influence of the perpendicular yarns in the fabric. A biaxial tensile test allows the stretching of the fabric in the two yarn directions at the same time, and hence, the determination of the tensile properties of the fabric due to a variety of in-plane-force combinations. In a first approximation, models generally do not account for the effect of the tension in the lateral direction, and that is why the simpler uniaxial testing of single yarns was chosen for the material characterization in this research.

The nonlinear tensile behavior of the fabric due to the decrimping is essential to perform reliable simulations [17]. If the crimp ratio (difference between the length of the yarn and the length of the fabric) is known, then the decrimping part of the stress-strain curve can be extrapolated from the experimental data.

The in-plane shear properties were obtained by a shear-frame test. In the speed range attainable with a conventional tensile machine, the fabric load-deformation response in the shear-frame test was not found to be rate dependent [3].

\subsection{Experimental Results of the Mechanical Behavior}

Three Twintex® woven fabrics composed of commingled polypropylene and glass fibers designed for thermostamping were tested [18]. The fabric characteristics can be found in [19]. PW, TWI and TWII refer to the plain-weave; the thin-twill-weave and the thick-twill-weave fabrics, respectively.

Uniaxial tensile testing was performed on an Instron 4464 machine with a $2-\mathrm{kN}$ load cell. Pneumatic cord and yarn grips were used, and the gauge length was set to approximately $1 \mathrm{~m}$ to minimize the effect of the deformation in the grips. The displacement rate was $5 \mathrm{~mm} / \mathrm{s}$.

The stiffness of the yarns was determined from the slope of the load/true-strain curves. In addition, measurements of the crimp ratio were performed. The results, presented in Table 1, were used to assess the tensile behavior of the yarn within the fabric via an extrapolation of the decrimping (Fig. 19).

Shear-frame tests were performed on the Instron 4464 machine. The geometry chosen for the specimen is shown in Fig. 20. Note that to avoid the shearing contribution of the arms, the cross yarns were removed from this part of the sample. The length of the frame $L_{F}$ was $216 \mathrm{~mm}$, and the length of the fabric $L_{f}$ was $120 \mathrm{~mm}$. 
Table 1: Yarn tensile stiffness [18]

\begin{tabular}{lll}
\hline Pattern & $\begin{array}{l}\text { Tensile stiffness } \\
(\mathrm{kN})\end{array}$ & $\begin{array}{l}\text { Crimp } \\
\text { ratio }(\%)\end{array}$ \\
\hline PW & $24.6 \pm 0.7$ & 2.0 \\
TWI (warp/weft) & $25.1 \pm 0.6 /$ & $4.4 / 0.8$ \\
TWII (warp/weft) & $50.3 \pm 0.4$ & $3.0 / 0.0$ \\
\hline
\end{tabular}

The specimen was sheared at the rate of $2 \mathrm{~mm} / \mathrm{s}$. A mechanical conditioning of five pre-test shearing runs was applied. The mechanical conditioning ensured a uniform distribution of the yarns across the sample. The normalized shear force $F_{s h}$ and the shear angle $\gamma$ were determined from the crosshead displacement $\delta$ and the total load on the frame $F$ by the following equations [19]:

$$
\begin{gathered}
\gamma=\frac{\pi}{2}-2 \cdot \arccos \left(\frac{\delta}{2 \cdot L_{F}}+\frac{\sqrt{2}}{2}\right) \\
F_{s h}=\frac{L_{F}}{L_{f}{ }^{2}} \frac{F}{2 \cos (\pi / 4-\gamma / 2)}
\end{gathered}
$$

Tests were performed at room temperature and at $175^{\circ} \mathrm{C}$, which is the recommended processing temperature for forming of these fabrics. The results are presented in Figs. 21 and 22.

The TWI fabric has a very tight weave and exhibits logically higher shear stiffness than the two other Twintex ${ }^{\circledR}$ fabrics. It is worth noting that the repeatability (represented by the error bars) is good up to the locking angle $\left(\sim 30^{\circ}\right.$ for TWI, $\sim 40^{\circ}$ for PW and TWII). At least five tests were used to obtain a reliable average.

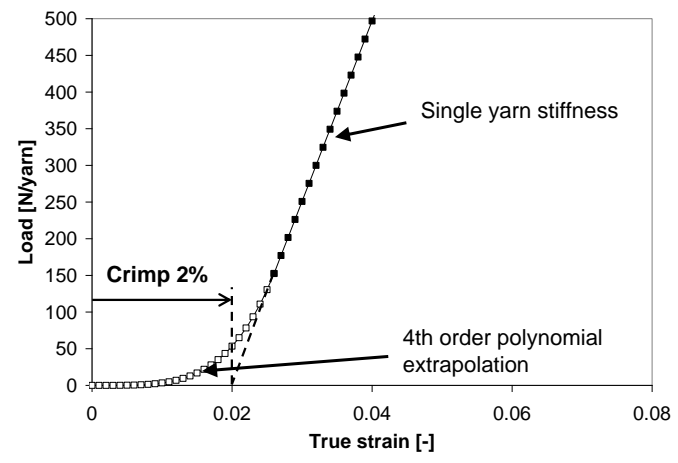

Fig. 19 Extrapolation of the fabric tensile behavior from the single yarn stiffness and the crimp ratio $(\mathrm{PW})$. 


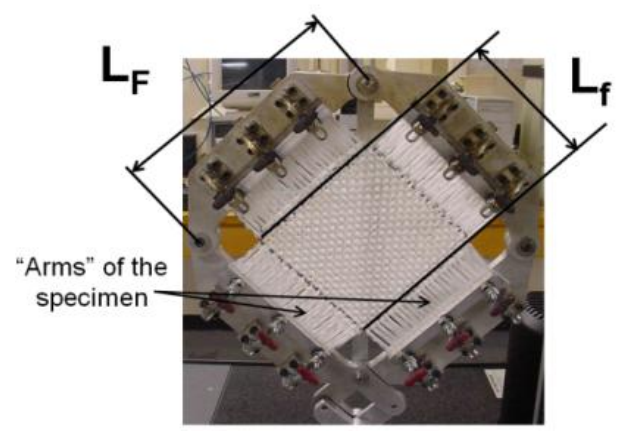

Fig. 20 Geometry of the shear frame. Free yarns have been removed in the "arms" of the specimen.

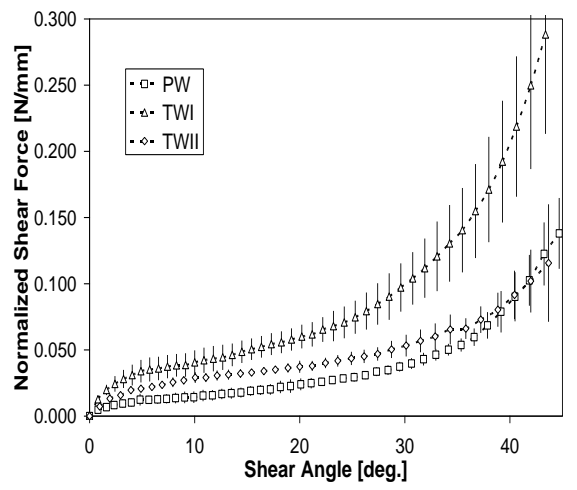

Fig. 21 In-plane shear behavior at room temperature [18].

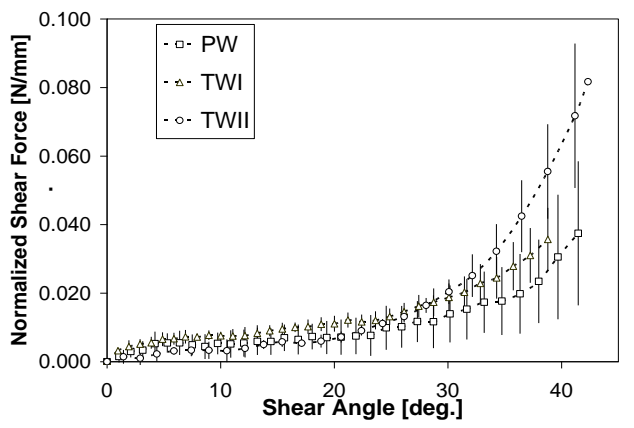

Fig. 22 In-plane shear behaviour at $175^{\circ} \mathrm{C}[18]$.

\subsection{Finite Element Modeling [20,21]}

Several modeling approaches have been developed to account for the evolution of the orthotropic directions during high shearing, and these approaches include the continuous approach, the semi-discrete finite element approach and the mesoscopic approach [22]. The first two approaches, while giving good results and being efficient in terms of computing time are generally somewhat complex and sometimes very challenging to implement into commercially available FEA packages. In the current research, a discrete mesoscopic approach, based on built-in elements and user-defined material and friction subroutines in commercially-available finite element codes was used. The explicit codes used were ABAQUS and LS-DYNA, and the material parameters were derived from conventional tensile and shear-frame experimental data as previously discussed in this report. 
The method was also implemented into ABAQUS/Standard (implicit solver) [23]. However, problems with convergence in ABAQUS/Standard due to the contact of the fabric with binders other than binder plates that covered all of the fabric could not be overcome. The elements needed for the methodology are not currently available in LS-DYNA implicit solver, so using the LS-DYNA implicit solver for the process simulation was not an option for this research.

\subsection{Finite Element Model Description}

The unit cell of the mesoscopic model used in this research for a plain-weave fabric consists of four 1-D elements and one 2-D element as shown in Fig. 23 for a plain-weave fabric. The 1-D elements carry the tensile loads through the fabric and are able to rotate freely as the fabric is sheared. This rotation of the 1-D elements is a simple and automatic way of accounting for the local changes in orientation of the principal load paths through the fabric. The 2-D element is included in the model to capture the in-plane shear stiffness of the fabric and has no tensile stiffness. The respective material models for the 1-D and 2-D elements were implemented in the finite element codes by userdefined subroutines. These material models capture the nonlinear load-displacement behavior of the fabric for inplane tensile and shearing deformations. Unit cells can likewise be defined by a combination of 1-D and 2-D elements for other weave patterns, such as satin and twill weaves [21,23].

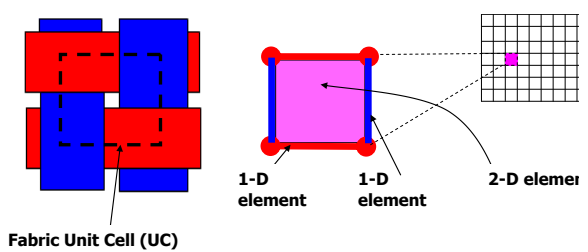

Fig. 23 Representative unit cell of the hybrid mesoscopic modeling of a plainweave fabric [23].

\subsection{Constitutive Equation for 1-D Elements}

The explicit finite element codes ABAQUS and LS-DYNA allow for customized stress-strain relationships at each time step via a user-defined material subroutine [24]:

$$
\Delta \sigma_{i j}^{t+1}=C_{i j k l} \cdot \Delta \varepsilon_{k l}^{t+1 / 2}
$$

where $\Delta \sigma_{i j}^{t+1}$ is the stress increment at time step $t+1, C_{i j k l}$ is the constitutive matrix defined by the user-supplied material parameters at time step $t$, and $\Delta \varepsilon_{k l}^{t+1 / 2}$ is the midpoint strain increment from the solver. For 1-D elements, Eq. (6) becomes:

$$
\Delta \sigma_{11}^{t+1}=C_{11}\left(\varepsilon_{11}\right) \cdot \Delta \varepsilon_{11}^{t+1 / 2}
$$

where $C_{11}$ is the tangent tensile modulus and is a function of $\varepsilon_{11}$. This tangent modulus is easily deduced from experimental tensile stress-true strain data of the fabric.

\subsection{Constitutive Equation for 2-D Elements}

The incorporation of the in-plane shear behavior in a shell/membrane element for finite-strain analysis is more challenging than the tensile behavior of the 1-D elements. Two reference frames as depicted in Fig. 24 have to be considered as the fabric shears from its initial orthogonal configuration to a nonorthogonal configuration. The $\boldsymbol{e}_{\boldsymbol{i}}$ unit vectors define the local orthogonal reference frame that rotates with the material, and the $\boldsymbol{g}_{\boldsymbol{i}}$ basis vectors form a nonorthogonal frame that follows the fiber directions: 


$$
\vec{g}_{i}=\underline{\underline{F}} \cdot \vec{g}_{i}^{0}=\underline{\underline{F}} \cdot \vec{e}_{i}^{0}
$$

where $\underline{\underline{F}}$ is the deformation gradient tensor known at each increment in the deformation process.

The in-plane shear-stress/shear-strain behavior of the woven fabric is captured by:

$$
\begin{aligned}
& \Delta \tilde{\sigma}_{12}^{t+1}=\tilde{C}_{33}\left(\gamma_{12}\right) \cdot \Delta \gamma_{12}^{t+1 / 2} \\
& \text { with } \Delta \gamma_{12}^{t+1 / 2}=2 \cdot \Delta \varepsilon_{12}^{t+1 / 2}
\end{aligned}
$$

where $\Delta \tilde{\sigma}_{12}^{t+1}$ is the shear-stress increment expressed in $g_{i}$ and $\widetilde{C}_{33}\left(\gamma_{12}\right)$ is the tangent shear modulus that can be determined from experimental data or by using the analytical method described earlier in this paper. This constitutive equation uses the tensorial shear-strain increment $\Delta \varepsilon_{12}^{t+1 / 2}$ expressed in the $\boldsymbol{e}_{\boldsymbol{i}}$ reference frame as it is given by the finite element solver to the user-defined material subroutine. However, the obtained stress increment is expressed in terms of the $\boldsymbol{g}_{\boldsymbol{i}}$ basis and needs to be returned to the finite element solver in the $\boldsymbol{e}_{\boldsymbol{i}}$ basis at the end of the user-defined subroutine. The rotation matrix relating the two reference frames can be obtained from a geometric analysis [25]. The 1-D elements carry the stresses in the $g_{1}$ and $g_{2}$ directions, and consequently $\Delta \tilde{\sigma}_{11}=\Delta \tilde{\sigma}_{22}=0$ in the $g_{i}$ reference frame within the 2-D element. Therefore, the stress increments are returned to the finite element solver in the $\boldsymbol{e}_{\boldsymbol{i}}$ basis using:

$$
\begin{aligned}
& \Delta \sigma_{11}=2 \cdot \cos \alpha \cos (\alpha+\theta) \cdot \Delta \tilde{\sigma}_{12} \\
& \Delta \sigma_{22}=2 \cdot \sin \alpha \sin (\alpha+\theta) \cdot \Delta \tilde{\sigma}_{12} \\
& \Delta \sigma_{12}=\sin (2 \alpha+\theta) \cdot \Delta \tilde{\sigma}_{12}
\end{aligned}
$$

where the $\Delta \sigma_{i j}$ are the stress increments in the $\boldsymbol{e}_{i}$ reference frame and the $\Delta \tilde{\sigma}_{i j}$ are the stresses in the $g_{i}$ reference frame. The angle between $\boldsymbol{e}_{1}$ and $\boldsymbol{g}_{\boldsymbol{1}}$ is $\alpha$, and the angle between $\boldsymbol{g}_{\boldsymbol{1}}$ and $\boldsymbol{g}_{2}$ is $\theta$.

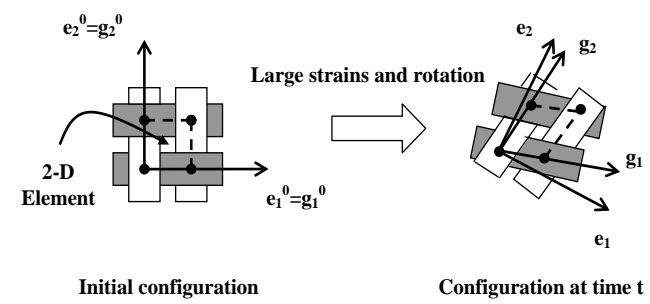

Fig. 24 Schematic representation of the reference frames used.

To be consistent with finite element codes that use the logarithmic strains for the summation of the strains increments, the logarithmic definition of strains should be used to determine $\widetilde{C}_{33}\left(\gamma_{12}\right)$ from the experimental data or analytical shear-frame model of the fabric. The true or "logarithmic" shear angle $\gamma^{L}$ can be obtained from the logarithmic strains in the principal stretch directions $\varepsilon_{I}^{L}$ and $\varepsilon_{I I}^{L}$ by a $45^{\circ} \mathrm{Mohr}$ 's circle transformation. In the case of a shear frame test, a geometrical analysis leads to the expression of $\gamma^{L}$ as a function of the geometric shear angle $\gamma:$

$$
\gamma^{L}=\varepsilon_{I}^{L}-\varepsilon_{I I}^{L}=-\ln (\tan (\pi / 4-\gamma / 2))
$$


The shear stress $\tilde{\sigma}_{12}$ is equal to the normalized shear force $F_{s h}[18]$ divided by the thickness of the fabric. Finally, $\tilde{C}_{33}\left(\gamma^{L}\right)$ is obtained from a derivation of $\tilde{\sigma}_{12}$ versus $\gamma^{L}$ of the experimental shear frame test data or using the analytical shear-frame model.

\subsection{Implementation via the User-Defined Material Subroutines}

The method has been implemented via user-defined material subroutines in the explicit finite element codes ABAQUS and LS-DYNA. For ABAQUS, B31 beam elements and S4R shell elements were used, and for LSDYNA seatbelt elements and Hughs-Liu reduced integration shells (Shell 6) were used. Truss elements can also be used in either ABAQUS or LS-DYNA for modeling of the yarns. However, for large and complex deformations, the convergence of the analysis was found to be difficult to achieve when using truss elements. The LS-DYNA seatbelt element is very efficient in terms of computational time and has the added benefit of having zero compressive stiffness. The modeling of the shear contribution to the material stiffness could potentially be captured using either shell or membrane elements with careful attention being given to the accuracy of the element response at high shear strains.

To demonstrate the capabilities of the methodology to simulate the manufacturing process for Twintex® woven fabrics, plain-weave experimental data at room and elevated temperatures were used to conclude the material parameters for the user-defined material subroutines used in the finite element simulations. The shear stress versus $\gamma^{L}$ curve was first developed from normalized experimental data:

- $\quad$ shear stress was assessed from normalized shear force $F_{s h}$ assuming a fabric thickness of $0.5 \mathrm{~mm}$, and

- $\quad \gamma^{L}$ was obtained from the geometrical shear angle $\gamma$ using Eq.(11).

The tangent shear modulus $\tilde{C}_{33}\left(\gamma^{L}\right)$ was then obtained by fitting a $4^{\text {th }}$-order polynomial to the shear stress vs. $\gamma^{L}$ experimental data.

Similarly the tangent tensile modulus was obtained from the tensile load vs. true strain experimental data. The "effective" section of the yarn $A_{\text {yarn }}$, with the assumption of no voids among the fibers was used. It was determined from the known data using the following equation [23]:

$$
A_{\text {yarn }}=\frac{\rho_{\text {yarn }}}{V F_{\text {glass }} \cdot \rho_{\text {glass }}+\left(1-V F_{\text {glass }}\right) \cdot \rho_{P P}}
$$

where $V F_{\text {glass }}$ is the glass volume fraction, $\rho_{\text {yarn }}$ is the yarn linear density, and $\rho_{\text {glass }}$ and $\rho_{P P}$ are the glass and polypropylene densities, respectively. The resulting $A_{\text {yarn }}$ is for the fabric being considered in the study. A square beam section was chosen for ABAQUS beams. However, other cross-section geometries (e.g., circle or oval) can be implemented should they be deemed more representative of the fabric. Because of the specific formulation of LSDYNA seatbelt elements, only the section area could be defined and no cross-section type could be described.

\subsection{Validation}

To validate the modeling approach, a shear frame test and a bias extension test were simulated at room temperature, and the results were compared to experimental data. A finite element model of the shear-frame test used at UMass Lowell was built, including the "arms" of the specimens [18]. The procedure used for completing the shear-frame testing required the removal of the free yarns in the arms of the specimens, so only 1-D elements were used in the model in the area of the arms (Fig. 25(a)). A bias-extension test was also simulated. The characteristic shear-strain field of this test [26] was obtained as shown in Fig. 25. 


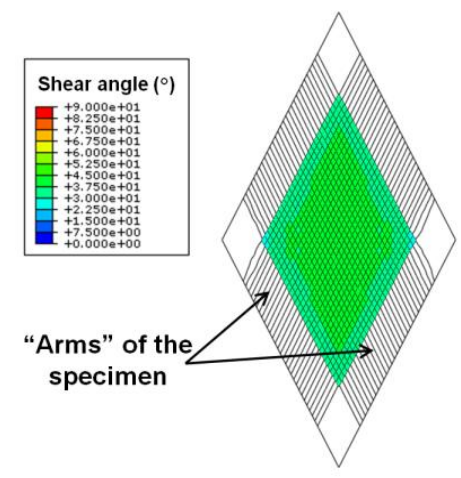

(a)

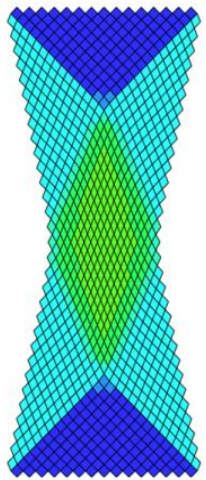

(b)

Fig. 25 Shear angle contour of (a) the shear frame and (b) the bias extension models (ABAQUS).

Figs. 26 and 27 show a comparison among finite element results from ABAQUS and LS-DYNA and experimental data to previous experimental work at UMass-Lowell (Shear frame data [18]) and to experimental data from Peng and Cao (Bias extension data [25]) for the Twintex ${ }^{\circledR}$ woven-fabric thermoplastic composite. Very good correlation was obtained between the ABAQUS and LS-DYNA results for both test conditions. The error bars in Fig. 26 denote one standard deviation. The simulations correlate well with the experimental data for the shear frame test, which validates the approach used, in particular the way the in-plane shear behavior is implemented. Concerning the biasextension test, experimental and simulation curves tend to diverge after $35 \mathrm{~mm}$ of displacement. This divergence could be a consequence of yarn sliding during the experiment [27] that is not considered in the simulation. This fact has been recently confirmed by experimental data obtained with a Digital Correlation Image technique used to track the shear deformation during the bias-extension test [21]. However, this sliding occurs at very large shear angles that may not be practical in the manufacture of quality parts using this composite forming process.

\subsection{Thermostamping Modeling}

The stamping of a hemisphere was simulated to demonstrate the capability of the approach for compound curvatures. A binder was used to induce in-plane tensile forces within the fabric to eliminate wrinkling. The two finite element codes give very similar results in terms of shear angle contours (Fig. 28). Although an actual thermostamped part is not shown here, these finite element results agree with what is observed in an actual part. For these simulations, the coefficient of friction was 0.3 .

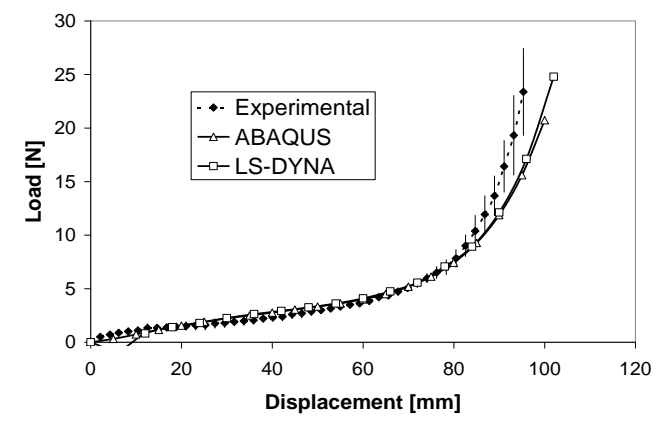

Fig. 26 Comparison of the load-displacement curves for the experimental and finiteelement simulations of the shear-frame test. 


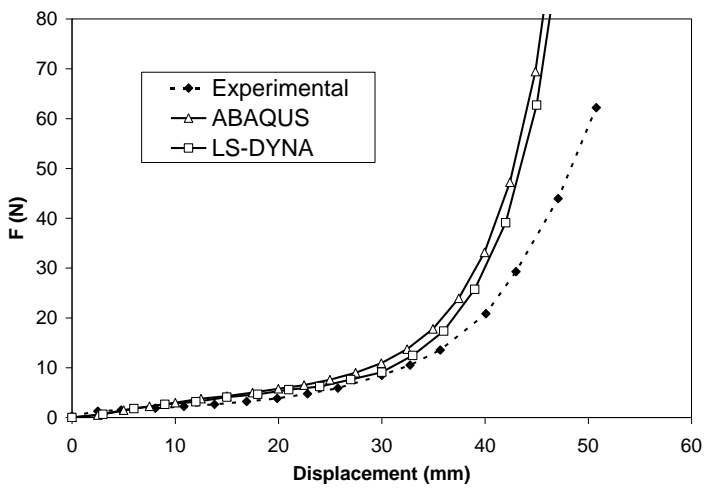

Fig. 27 Comparison of the load-displacement curves for the experimental and finiteelement simulations of the bias-extension test.

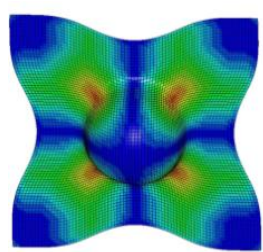

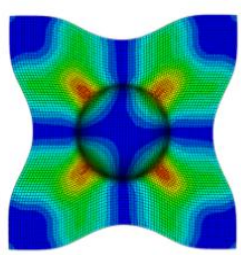

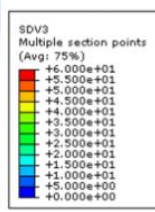

Fig. 28 Shear angle contour comparison between (a) LS-DYNA and (b) ABAQUS simulations.

\subsection{Industrial Application of the Design Tool}

The proposed simulation methodology was also applied to the forming of a composite tub for the automotive industry [28]. The fabric used for these simulations was a plain-weave fabric compounded with a thermoset resin which is pliable at room temperature. This fabric was characterized experimentally using tensile, shear-frame and friction tests. The friction was determined by the $3^{\text {rd }}$-generation friction testing apparatus to be essentially zero due to the lubrication effect resulting from the compounding material. The woven-fabric material properties were incorporated in the finite element model to simulate the forming process. The resulting parts are shown in Fig. 29.

For the tub shown in Fig. 29, the top contour plot shows the resulting shear-strain distribution across the part for a $0^{\circ} / 90^{\circ}$ ply orientation, and the bottom is for a $\pm 45^{\circ}$ ply orientation. The shear contours range from $0^{\circ}$ to $60^{\circ}$ as the color contours span from blue to red, respectively. Any shear angles above $60^{\circ}$ are shown in white. Note how the $0^{\circ} / 90^{\circ}$ ply is blue over a majority of the part. As a result, there is very high shearing in the corners to allow the fabric to assume the shape of the tub. This high shearing of greater than $60^{\circ}$ is associated with localized wrinkling. By rotating the fabric $45^{\circ}$ as shown in the bottom contour plot, the shearing can be distributed across the part and thereby avoid locally high shearing which can result in wrinkling. 

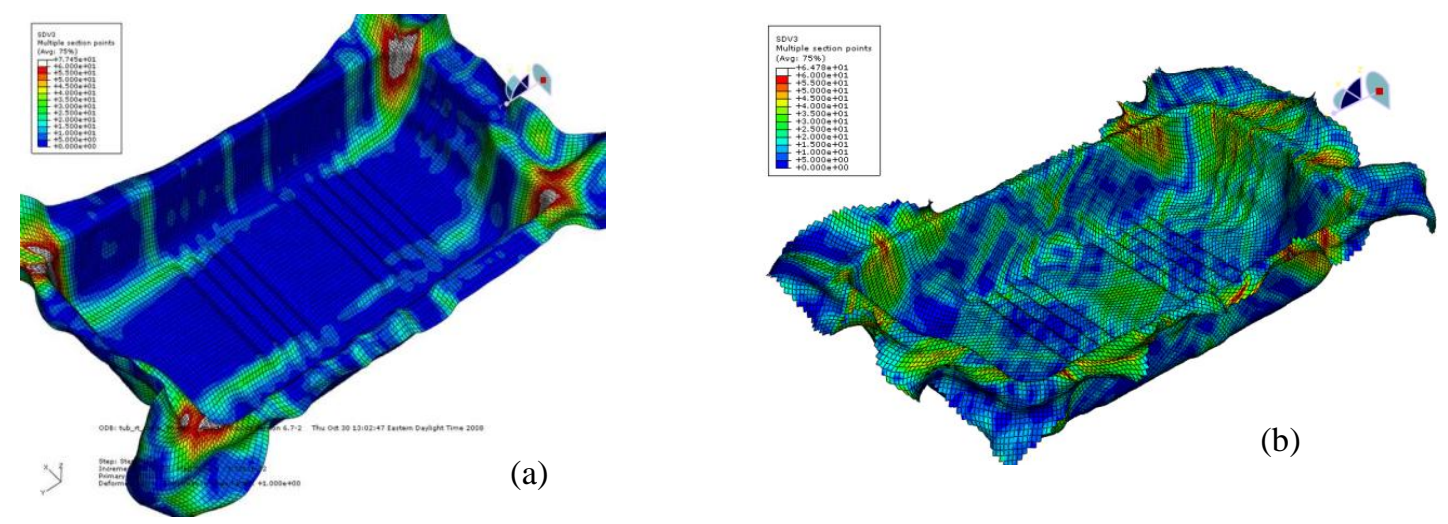

Fig. 29 Forming simulation of the Tub for a $0^{\circ} / 90^{\circ}$ ply orientation (a) and a $\pm 45^{\circ}$ (b). Shear angle contour (blue to red $=0^{\circ}$ to $60^{\circ}$, white above $60^{\circ}$ ).

This simulation of the tub forming process using the design tool developed in this NSF-funded research was completed as part of an ACC (Automotive Composites Consortium) research project for USCAR. The objectives of the simulations were to give insight as to problematic areas that may occur during the manufacturing process, i.e. wrinkling that could comprise the quality of the composite tub, and what processing steps may be taken to alleviate these problematic areas. In addition, by using the design tool to look at the forming of the tub using multiple layers of fabrics with varying orientations, the resulting structural stiffness of the composite tub can be accessed. Thus, the technological advances coming from this NSF research are being implemented by the US automotive industry to advance the use of composites in automotive designs leading to lighter and more fuel efficient cars and in reducing the cost of manufacturing composite parts.

Because of the success in applying the results of this composite forming simulation tool to the USCAR project, the UMass-Lowell team has been invited to collaborate with MAG-Industrial Automated Systems (formerly Cincinnati Machine) to explore the potential to form composite parts for the automotive industry using prepreg unidirectional tapes and woven fabrics. A joint proposal to NCMS (National Center for Manufacturing Science) is currently being written for submission for a January 2010 project start date.

\subsection{International Benchmarking Program}

In an effort to understand the strengths and limitations of the different simulation methods that are being developed by various research teams around the world, an international benchmark survey was conducted. Several groups submitted simulation results for the thermostamping of plain-weave Twintex fabric using a double-dome geometry as shown in Fig 30. Comparisons were made by observing the resulting draw-in of the fabric and shear angles developed in the fabric after stamping. As a result, a standard set of testing parameters was developed for a future round of simulations for comparison among the research groups.



Fig. 30 Finite element mesh of the Double-Dome Geometry 


\subsection{Materials and Geometry}

The following bullets list the items to be used in the next round of simulations amongst the various international research teams:

- Double-dome tool (Fig. 30)

- Twintex fabrics (Plain and Fat-Twill weaves)

- Alternate fabrics tested and formed in participant labs

All simulation results will be compared to those of formed parts that were completed at Ford Research Labs in 2004 using the fat-twill fabric.

\subsection{Simulation Parameters}

After analyzing and comparing simulation results from several groups after a first round of simulations by the respective research groups, a uniform set of simulation parameters for study has been determined for use in a second round of simulations. These parameters include the orientation of the material blank, the amount of binder force applied and the type of material being simulated. The orientation of the fiber blank will be varied between the $0 / 90^{\circ}$ and $\pm 45^{\circ}$ orientations. The amount of binder force applied will be varied between a very low binder force (close to 0 ) and a large amount of force $(1000 \mathrm{~N})$. The materials being simulated will be characterized by two different sets of tensile and shear stress curves which will be provided to all groups based on the UMass-Lowell test data.

Other parameters defined in the simulations will be set at specific values for all groups. These are parameters that had less effect on the simulation results from Round 1 than the parameters previously discussed and include the coefficient of friction and the size of the material blank. The coefficient of friction will be set at 0.3 because no other team other than the UMass-Lowell is known to have the ability to account for variable friction. The material blank size will be set at $300 \mathrm{~mm}$ x $450 \mathrm{~mm}$. The parameters for each simulation are presented in Table 2 and the standard parameter values are summarized in Table 3. In Table 2, PW denotes plain weave and FT denotes fat twill weave.

Table 2: Simulation parameter matrix.

$\begin{array}{cccc}\text { Simulation } & \text { Mat'1 } & \begin{array}{c}\text { Blank } \\ \text { Orientation } \\ 0 / 90^{\circ}\end{array} & \begin{array}{c}\text { Binder } \\ \text { Force }(\mathrm{N})\end{array} \\ 1 & \text { PW } & 0 \\ 2 & \text { PW } & 0 / 90^{\circ} & 1000 \\ 3 & \text { PW } & \pm 45^{\circ} & 0 \\ 4 & \text { PW } & \pm 45^{\circ} & 1000 \\ 5 & \text { FT } & 0 / 90^{\circ} & 0 \\ 6 & \text { FT } & 0 / 90^{\circ} & 1000 \\ 7 & \text { FT } & \pm 45^{\circ} & 0 \\ 8 & \text { FT } & \pm 45^{\circ} & 1000\end{array}$

Table 3: Constant simulation parameters.

$\begin{array}{ccc}\text { Parameter } & \begin{array}{c}\text { Coefficient } \\ \text { of Friction }\end{array} & \begin{array}{c}\text { Blank Size } \\ (\mathrm{mm})\end{array} \\ \text { Value } & 0.3 & 300 \times 450\end{array}$

\subsection{Comparison Metrics}

Comparison of simulation results will rely on the same metrics used in the first round of comparisons. These include the amount of fabric draw-in at three specified points as well as the shear angles reported along the apex. The locations of the draw-in points are illustrated in Fig. 31. All points of draw-in are located on the edge of the material blank. D1 and D3 are located at the center point of their respective sides. D2 is located at the corner of the material blank. 


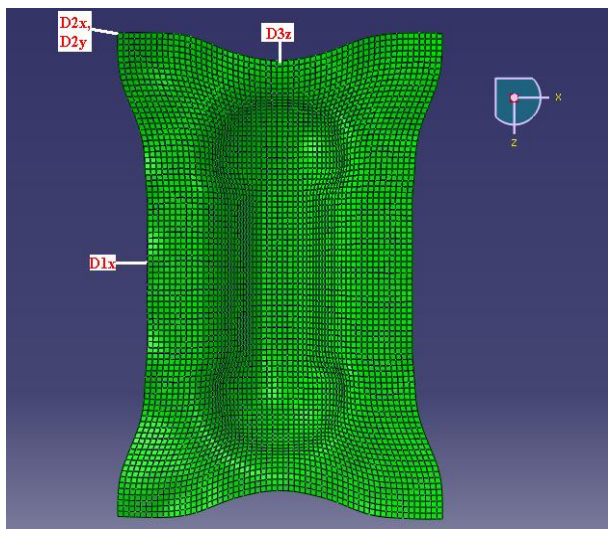

Fig. 31 Location of reported draw-in.

Shear angles are to be reported along the apex. The apex is defined as the line which passes through the points of greatest shear. Fig. 32 illustrates the location of the apex with a red line. The line begins at the vertical axis of symmetry and extends through the location of greatest shear angle all the way to the edge of the material blank.

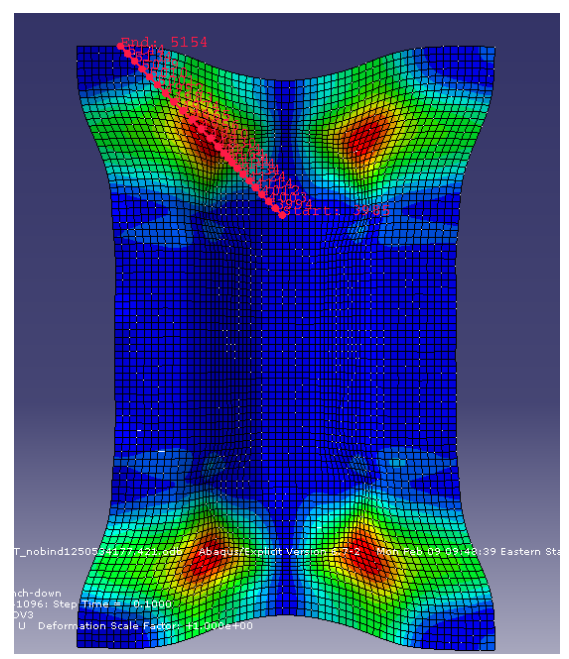

Fig. 32 Shear angle are to be measured along the apex line shown in red.

\subsection{Participants}

Table 4 is a list of participants for the next round of simulations.

Table 4. Potential Participants

\begin{tabular}{|c|c|c|}
\hline Name & Institution & Email \\
\hline Jian Cao & Northwestern Univ, USA & jcao@northwestern.edu \\
\hline James Sherwood & Univ of MA - Lowell, USA & James_Sherwood@uml.edu \\
\hline Philippe Boisse & INSA-Lyon, FRANCE & Philippe.Boisse@insa-lyon.fr \\
\hline Remko Akkerman & Univ of Twente, NETHERLANDS & r.akkerman@ctw.utwente.nl \\
\hline Stepan Lomov & KU-Leuven, BELGIUM & Stepan.Lomov@mtm.kuleuven.be \\
\hline Andy Long & Univ of Nottingham, UK & Andrew.Long@nottingham.ac.uk \\
\hline Tong-Xi Yu & HKUST, HONG KONG & metxyu@ust.hk \\
\hline
\end{tabular}




\subsection{Friction Modeling}

Parametric studies of the thermostamping process using the Hersey number friction model and different binder forces have been completed to explore the effect of the in-plane tension on the wrinkling and tearing of the fabric. These studies demonstrated how the model could be used to determine the most appropriate binder force to avoid wrinkling (high shear strain within the fabric) and tearing (high tensile stress in the yarns).

The phenomenological friction models relating the dynamic coefficient of friction to the Hersey number were incorporated into ABAQUS/Explicit and LS-DYNA as user-defined friction subroutines in conjunction with userdefined material models. The user-defined friction subroutines were first validated using a finite element model of the experimental friction test. The dynamic coefficients of friction obtained from the finite element simulations correlated well with the experimentally obtained dynamic coefficients of friction. Static coefficients of friction were not included in the friction subroutines after a parametric study showed that the importance of the static friction coefficient contribution to the overall result is of minimal significance in comparison to the contribution of the dynamic coefficient of friction. The user-defined friction subroutines accounted for dynamically changing coefficients as a function of variations in velocities and normal forces experienced by the fabric.

It was found that capturing varying friction at the tool/fabric and fabric/fabric interfaces was important because the varying friction significantly affected the force required by the punch to form the part, as well as the resulting tensile stresses in the fabric yarns. A parametric study showed that increasing the stamping rate led to an increase in the friction force at the tool/fabric interface, thus increasing the punch force and the tensile stresses in the yarns. Using a constant coefficient of friction showed that the punch force had no dependence on the stamping rate. Incorrectly predicting the fabric stresses could lead to manufacturing defects such as fabric tearing and not optimizing the processing speed, i.e. manufacturing rate.

After validating the user-supplied subroutines with a finite element model of the friction test, both VFRIC (ABAQUS) and USRFRC (LS-DYNA) were applied to a single-layer $\left(\left[0^{\circ} / 90^{\circ}\right]\right)$ hemisphere model with equal binder pressures and stamping rates, and the resulting punch forces were compared between ABAQUS/Explicit and LS-DYNA. Figure 33 shows that the results from the ABAQUS and LS-DYNA agree well with each other.

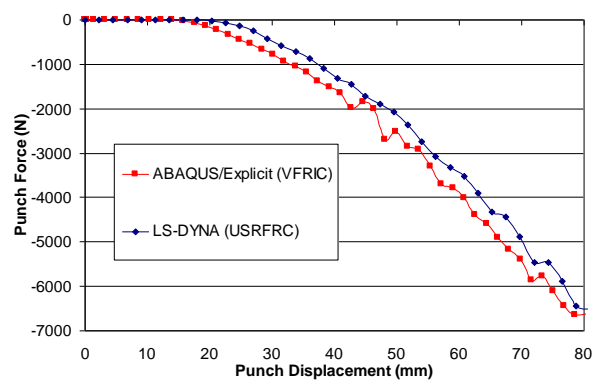

Fig. 33 Comparison of hemisphere model between ABAQUS and LS-DYNA for user-defined friction subroutines with a binder pressure of $200 \mathrm{kPa}$ and a stamping rate of $90 \mathrm{~mm} / \mathrm{s}$

It was found that as the friction increases the punch force required to deform the fabric will increase. From the experimental results, it was shown that as the velocities increase the frictional forces between the metal tooling and the fabric and between adjacent layers of fabric will increase. However, as the normal force between the fabric and the contacting surface increases, the effective friction coefficient decreases. Thus, it is expected that as the stamping rate is increased, then the punch forces required to deform the fabric may increase or decrease depending on the combination of speed and normal force. To explore the effect of stamping rate on the punch force, three different stamping rates were applied to the hemisphere model in ABAQUS/Explicit. Table 5 summarizes the stamping rates used, and Fig. 34 compares the punch forces at each stamping rate as a function of punch displacement. The differences in punch forces shown in Fig. 34 indicate that the punch force does increase with increasing punch velocity. 
Table 5. Effect of punch velocity on punch force

$\begin{array}{ccc}\begin{array}{c}\text { Simulation } \\ \text { No. }\end{array} & \begin{array}{c}\text { Binder } \\ \text { Pressure } \\ (\mathrm{kPa})\end{array} & \begin{array}{c}\text { Punch } \\ \text { Velocity } \\ (\mathrm{mm} / \mathrm{s})\end{array} \\ 1 & 200 & 9.0 \\ 2 & 200 & 22.5 \\ 3 & 200 & 45.0\end{array}$

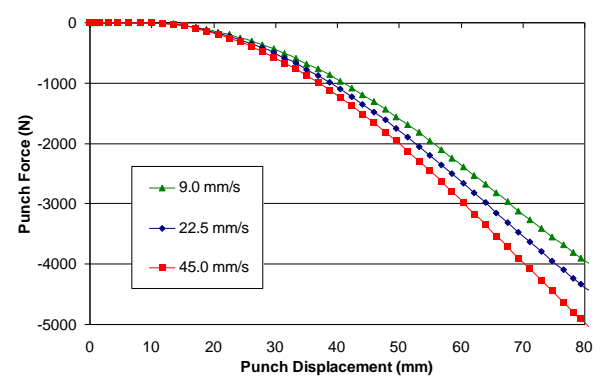

Fig. 34 Effect of punch velocity on punch force as a function of displacement using fabric-friction model

Using the same stamping rates and binder pressures as given in Table 5 with a fixed coefficient of friction of 0.3 , the punch forces show no dependence on stamping rate. Thus, if the modeling is done using a constant coefficient of friction, the punch force can be either over-predicted or under-predicted relative to the results shown using a variable coefficient of friction, i.e. is a function of the velocity (Fig. 35). Fig. 35 shows the importance of incorporating a varying friction coefficient that is dependent upon the critical thermostamping process parameters such as stamping rate. As stated previously, higher frictional forces also increase the tensile stresses in the yarns in addition to the punch force. Fig. 36 shows that assuming a constant coefficient of friction $(\mu=0.3)$ greatly overpredicts the tensile stresses in the fabric yarns. Incorrectly predicting the fabric stresses could lead to manufacturing defects such as fabric tearing and not optimizing the processing speed, i.e. manufacturing rate.

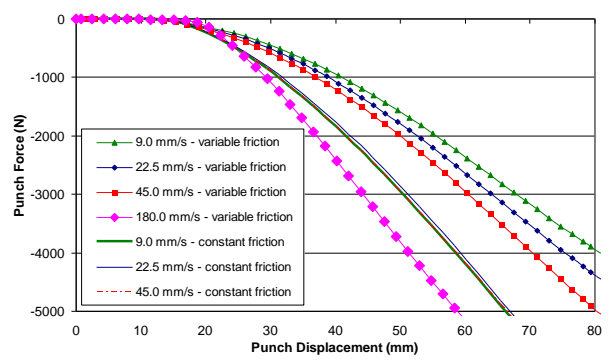

Fig. 35 Punch forces with the variable fabricfriction model and with constant friction coefficient

$(\mu=0.3)$ 

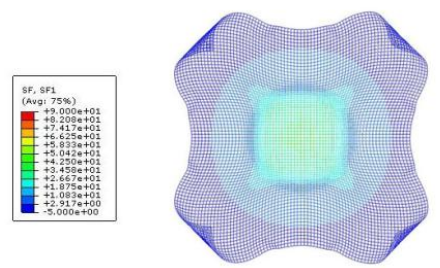

(a)

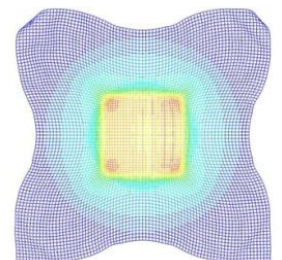

(b)

Fig. 36 Tensile stresses in fabric yarns with (a) fabric-friction model and with (b) constant friction coefficient $(\mu=0.3)$

\subsection{Modal Analysis}

Modal analysis is currently being pursued as a means to quantify the correlation between the thermostamping simulation and actual parts made using the process. The modal analysis is using the 3D Laser Vibrometer which was acquired with the funding made possible by the NSF Major Research Initiation Grant CMMI-0722469. The study is being done for flat plates where the fabric has the yarns in their as-fabricated state, i.e. where warp and weft are mutually perpendicular, and in a sheared state, e.g. sheared $25^{\circ}$ from the as-fabricated weave orientation of $90^{\circ}$. For a three-dimensional shape, the double-dome geometry as shown is Fig. 30 is being used.

Some initial comparisons between the composite formed using the simulation and actual formed parts have been completed and are showing some promising results. The mode shapes and frequencies for the first and second modes are shown in Figs. 37 and 38, respectively. The frequencies calculated from the model are very close to the experimentally measured values. However, as can be seen in Fig. 39, there is a wide discrepancy between the model and the experimental frequencies for the third mode, which is the fourth mode in the model. The difference is most likely due to the bending stiffness being used for the beam elements in the hybrid mesh. The bending stiffness is being explored using a parametric study.

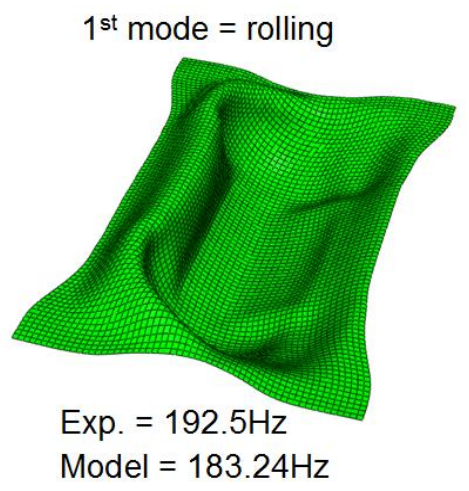

Fig. 37 Double Dome $1^{\text {st }}$ mode 


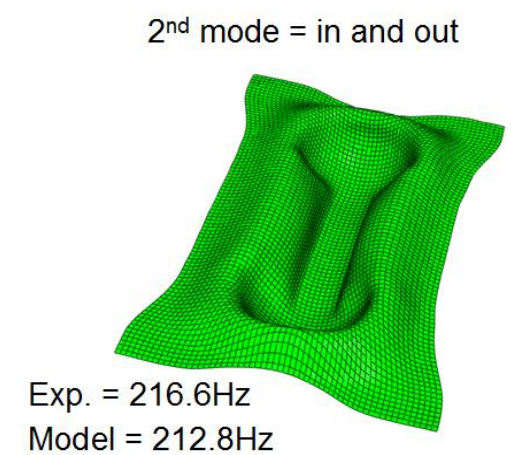

Fig. 38 Double Dome $2^{\text {nd }}$ mode

In and out + back and forth

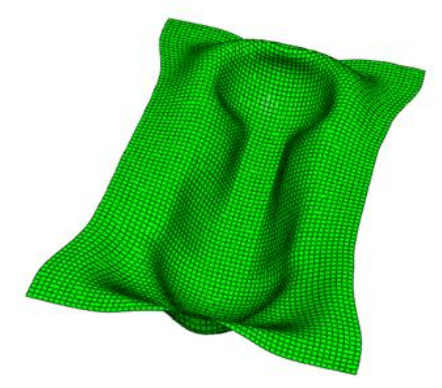

Exp. $($ mode 3$)=257.5 \mathrm{~Hz}$

Model $($ mode 4$)=347.8 \mathrm{~Hz}$

Fig. 39 Double Dome $3^{\text {rd }}$ mode 


\subsection{Publications}

1. Gamache L., Sherwood J. A., Chen J., Cao J.: Characterization of the Fabric/Tool and Fabric/Fabric Friction during the Thermostamping Process. Proceedings of the 10th International ESAFORM Conference on Material Forming. Zaragoza, Spain, 2007.

2. Gamache L.: The Design and Implementation of a Friction Test Apparatus Based on the Thermostamping Process of Woven-Fabric Composites, MS Thesis, Department of Mechanical Engineering. Lowell, MA: University of Massachusetts Lowell, 2007.

3. Cao J., Akkerman R., Boisse P., Chen J., Cheng H. S., DeGraaf E. F., Gorczyca J., Harrison P., Hivet G., Launay J., Lee W., Liu L., Lomov S., Long A., Deluycker E., Morestin F., Padvoiskis J., Peng X. Q., Sherwood J., Stoilova T., Tao X. M., Verpoest I., Willems A., Wiggers J., Yu T. X., Zhu B.: Characterization of mechanical behavior of woven fabrics: experimental methods and benchmark results. Composites: Part A, 39:1037-1053, 2008.

4. Fetfatsidis K. A., Sherwood J. A., Chen J., Jauffres D.: Characterization of the fabric/tool and fabric/fabric friction during the thermostamping process. Proceedings of the 12th International Conference. Twente, Nederlands, 2009.

5. Jauffres D., Morris C. D., Sherwood J., Chen J.: Simulation of the thermostamping of woven composites: determination of the tensile and in-plane shearing behaviors. Proceedings of the 12th International ESAFORM Conference. Twente, Nederlands, 2009.

6. Jauffres D., Morris C. D., Kremer J., Sherwood J., Chen J. Simulation of the thermostamping of woven composites: mesoscopic modeling using FEA explicit codes. Proceedings of the 12th International ESAFORM Conference. Twente, Nederlands, 2009.

7. Jauffres D., Morris C. D., Sherwood J., Chen J.: Discrete mesoscopic modeling for woven fabric reinforcement forming simulation. International Journal of Material Forming: (accepted for publication), 2009.

8. Jauffres D., Sherwood J. A., Chen J., Morris C. D., and Kremer J.: Mesosocopic finite element modeling of woven reinforcements applied to sheet molding compound forming simulation. Proceedings of the 17th International Conference on Composite Materials. Edinburgh, UK, 2009.

9. Fetfatsidis, K.A.: Characterization of the Tool/Fabric and Fabric/Fabric Friction for Woven Fabrics: Static and Dynamic, MS Thesis, Department of Mechanical Engineering. Lowell, MA: University of Massachusetts Lowell, 2009.

10. Morris, C.D.: Simulation of the Thermostamping Process for Woven Fabric Composites using LS-DYNA, MS Thesis, Department of Mechanical Engineering. Lowell, MA: University of Massachusetts Lowell, 2010 (estimated).

\subsection{Future Work}

More modal testing of a stamped part will be performed using a 3D laser vibrometer. Changes in the bending stiffness of the yarns and in the resulting ply thicknesses will be considered to improve the correlation between the model and the resulting part stiffness. This final exercise will show the ability of the modeling approach to provide useful data for subsequent structural analysis in addition to the information on the drapability.

\subsection{Conclusions}

Yarn and fiber parameters were investigated and their ability to model the compaction behavior of commingled fibers has been demonstrated by their influence on the resulting shear load-deformation curve. A $3^{\text {rd }}$-generation friction-test apparatus using load-control to apply the normal force to the surface of the fabric has been designed, built and validated allowing for the static and dynamic coefficients of friction to be quantified for tool-fabric and fabric-fabric friction. A set of experiments has been developed to quantify the mechanical behavior of woven fabrics for the forming process. A set of constitutive models has been implemented in the ABAQUS and LS-DYNA explicit finite element codes. The ability of the numerical design tool to simulate the forming of composite parts for woven fabric composites has been demonstrated. The UMass-Lowell research group is participating in an international benchmark program with other research groups doing similar work in Europe and Asia. The design tool is now being used to validate its ability to link the manufacturing process to the structural response of the formed part. This 
validation will be done by comparing experimental and finite element results for structural stiffness and vibration response of a relatively complex part.

\subsection{Acknowledgements}

The authors would like to thank the NSF Division of Design, Manufacture, and Industrial Innovation (DMI\#0522923), U.S. Department of Energy, and Ford Motor Company (Dr. Patrick Blanchard) for their support of this research. The letter of support from General Motors for the grant proposal is also appreciated. The contributions of Dr. Lu Liu, UMass-Lowell graduate and post doc, MSME students: Lisa Gamache, Corey Morris, Konstantine Fetfatsidis, and James Kremer, and undergraduate students David Winchester, Joanna Langworthy and Joseph Sargent and Ezekial Radik are appreciated. The expertise of post doc Dr. David Jauffrès is especially appreciated in the completion of the overall project.

\subsection{References}

1. C.E. Wilks, "Characterization of the Tool/Ply Interface during Forming," PhD Thesis, School of Mechanical, Materials, Manufacturing and Management. Nottingham, UK: University of Nottingham, 1999.

2. A.E. Long, C.D. Rudd, M. Blagdon M., and P. Smith, "Characterizing the Processing and Performance of Aligned Reinforcements during Preform Manufacture,” Composites: Part A, pp. 27247-253, 1996.

3. L. Liu, J. Chen, J. Gorczyca, J. Sherwood, "Modeling of Friction and Shear in Thermostamping of Composites Part II,” Journal of Composite Materials, vol. 38, pp. 1931-1947, 2004.

4. L. Liu, J. Chen, X. Li, and J. Sherwood, "Two-Dimensional Macro-Mechanics Shear Models of Woven Fabrics," Composites: Part A, vol. 36, pp.105-114, 2005.

5. L. Liu, J. Chen, J. Sherwood, "Analytical Model of Shear of 4-Harness Satin Weave Fabrics," American Institute of Physics Proceedings 712, 8th NUMIFORM Technical Conference, June 13-17, Columbus, OH, 2004.

6. A. Bulusu, "Modeling of Architecture and Deformation of Dry Woven Fabrics during Shear," MS Thesis, Department of Mechanical Engineering, Lowell, MA, University of Massachusetts Lowell, 2001.

7. I.M. Hutchings, Tribology: Friction and Wear of Engineering Materials, Ann Arbor, MI: CRC Press, 2002.

8. J. Gorczyca, "A Study of the Frictional Behavior of a Plain-Weave Fabric during the Thermostamping Process," DEng Dissertation, Department of Mechanical Engineering, Lowell, MA, University of Massachusetts Lowell, 2004.

9. J. Gorczyca-Cole, J.A. Sherwood and J. Chen, "A Friction Model for Thermostamping Commingled GlassPolypropylene Woven Fabrics,” Composites: Part A, vol. 38, pp. 393-406, 2007.

10. J. Gorczyca, J. Sherwood, L. Liu, and J. Chen, "Modeling of Friction and Shear in Thermostamping Process Part I,” Journal of Composite Materials, vol. 38, pp. 1911-1929, 2004.

11. S. Chow S., "Frictional Interaction between Blank Holder and Fabric in Stamping of Woven Thermoplastic Composites.," MS Thesis, Department of Mechanical Engineering, Lowell, MA: University of Massachusetts Lowell, 2002.

12. L. Gamache, "The Design and Implementation of a Friction Test Apparatus Based on the Thermostamping Process of Woven-Fabric Composites," MS Thesis, Department of Mechanical Engineering, Lowell, MA, University of Massachusetts Lowell, 2007.

13. L. Gamache, J.A. Sherwood, J. Chen, J. Cao, "Characterization of the Fabric/Tool and Fabric/Fabric Friction during the Thermostamping Process," Proceedings of the 10th International ESAFORM Conference on Material Forming, Zaragoza, Spain, 2007.

14. Fetfatsidis K. A., Sherwood J. A., Chen J., Jauffres D.: Characterization of the fabric/tool and fabric/fabric friction during the thermostamping process. Proceedings of the 12th International Conference on Material Forming. Twente, Nederlands, 2009. 
15. Fetfatsidis, K.A.: Characterization of the Tool/Fabric and Fabric/Fabric Friction for Woven Fabrics: Static and Dynamic, MS Thesis, Department of Mechanical Engineering. Lowell, MA: University of Massachusetts Lowell, 2009.

16. Morris, C.D.: Simulation of the Thermostamping Process for Woven Fabric Composites using LS-DYNA, MS Thesis, Department of Mechanical Engineering. Lowell, MA: University of Massachusetts Lowell, 2009.

17. Willems A., Lomov S., Vandepitte D., Verpoest I.: Double dome forming of woven textile composites. Proceedings of the 9th International ESAFORM Conference on Material Forming. Glasgow, UK, 2006.

18. Jauffres D., Morris C. D., Sherwood J., Chen J.: Simulation of the thermostamping of woven composites: determination of the tensile and in-plane shearing behaviors. Proceedings of the 12th International ESAFORM Conference on Material Forming. Twente, Nederlands, 2009.

19. Cao J., Akkerman R., Boisse P., Chen J., Cheng H. S., DeGraaf E. F., Gorczyca J., Harrison P., Hivet G., Launay J., Lee W., Liu L., Lomov S., Long A., Deluycker E., Morestin F., Padvoiskis J., Peng X. Q., Sherwood J., Stoilova T., Tao X. M., Verpoest I., Willems A., Wiggers J., Yu T. X., Zhu B.: Characterization of mechanical behavior of woven fabrics: experimental methods and benchmark results. Composites: Part A, 39:1037-1053, 2008.

20. Jauffres D., Morris C. D., Kremer J., Sherwood J., Chen J. Simulation of the thermostamping of woven composites: mesoscopic modeling using FEA explicit codes. Proceedings of the 12th International ESAFORM Conference on Material Forming. Twente, Nederlands, 2009.

21. Jauffres D., Morris C. D., Sherwood J., Chen J.: Discrete mesoscopic modeling for woven fabric reinforcement forming simulation. International Journal of Material Forming: (in publication), 2009.

22. Boisse P., Hamila N., Helenon F., Hagege B., Cao J.: Different approaches for woven composite reinforcement forming simulation. International Journal of Material Forming, 1:21-29, 2008.

23. Li X.: Material characterization of woven-fabric composites and finite element analysis of the thermostamping process. DEng Dissertation, Department of Mechanical Engineering. Lowell, MA: University of Massachusetts Lowell, 2005.

24. Bathe K. J.: Finite Element Procedures. Englewood Cliffs: New Jersey: Prentice Hall, 1996.

25. Peng X. Q., Cao J.: A continuum mechanics-based non-orthogonal constitutive model for woven composite fabrics. Composites: Part A, 36:859-874, 2005.

26. Lomov S., Boisse P., Deluycker E., Morestin F., Vanclooster K., Vandepitte D., Verpoest I., Willems A.: Fullfield strain measurements in textile deformability studies. Composites: Part A, 39:1232-1244, 2008.

27. Harrison P., Clifford M. J., Long A.: Shear characterization of viscous woven textile composites: a comparison between picture frame and bias extension experiments. Composites Science and Technology, 64:1453-1465, 2004.

28. Jauffres D., Sherwood J. A., Chen J., Morris C. D., and Kremer J.: Mesosocopic finite element modeling of woven reinforcements applied to sheet molding compound forming simulation. Proceedings of the 17th International Conference on Composite Materials. Edinburgh, UK, 2009. 
NSF GRANT \# DMII-0522923

NSF PROGRAM NAME: MPM

FINAL Report:

Linking Process-Induced Properties to Thermoplastic-Matrix Woven-Fabric Composites Performance

\author{
James Sherwood \\ Julie Chen \\ Larissa Gorbatikh \\ University of Massachusetts Lowell
}

\begin{abstract}
To model the in-service mechanical behavior of thermoplastic-matrix woven-fabric reinforced structural composites, the mechanical properties resulting from the manufacturing process need to be known. This report will discuss the research tasks that have been completed through the fourth year of a research effort to develop a design tool which links the process-induced mechanical properties of thermoplastic-matrix woven-fabric composites to their in-service performance. To study the friction relationship between the forming tool and the deformed fabric geometry, the design and application of a novel test method is presented. Modal analysis is used to examine the correlation between the structural stiffnesses of the forming simulation to that of a complex three-dimensional formed geometry.
\end{abstract}

\title{
1.0 Introduction
}

Modeling of the in-service performance of thermoplastic-matrix woven-fabric-reinforced structural composites e.g., damage tolerance, crashworthiness, vibration - is currently inadequate for parts of any geometric complexity because of the inability of existing performance models to capture the true deformed material properties. As a result, lightweight composite materials are utilized inefficiently or not at all; alternatively, extensive experimental trial and error or design of experiments must be conducted to develop a satisfactory product. The key to addressing this barrier is to provide a direct link among part geometry, material selection, process conditions, process-induced local properties, and part performance, allowing informed feedback to the design process. Fiber-reinforced thermoplastic composites have a variety of applications including structural components in the automotive, aerospace, marine, infrastructure and recreation industries. The main advantage of these composites over metals is their high specific strength. They also have other beneficial properties including low thermal expansion and good corrosion resistance when compared to metals. Woven fabrics offer many other advantages when compared to metals in terms of deformation capabilities, including dimensional stability, good conformability, and deep-draw shapability. Compared to nonwoven-fabric composites, the woven-fabric composites provide more balanced properties, higher impact resistance, easier handling and lower fabrication cost, particularly for parts with complex shapes.

The objective of this research is to utilize an integrated analytical, experimental, and numerical effort to attain a fundamental understanding of two governing physical phenomena - interlayer (fabric-fabric) friction and interconnected through-thickness and in-plane compaction. The former is important in multiple-layer and multiplestep forming, both needed for industrial processes. The latter is a critical factor affecting the final part thickness, void content, fiber orientation, and fiber distribution - all are important for structural stiffness and damage tolerance. To validate and demonstrate the capabilities of the integrated structure-process-performance design tool, the model will be used to identify critical process and material parameters for one particular performance condition - vibration.

A combined inter-tow friction model, developed in a previous NSF-funded research program (DMII-0331267), based on the equilibrium equations of the unit cell of a balanced plain-weave glass/polypropylene woven fabric is extended to account for fiber compaction. To accomplish this extension of the model, fiber-compaction experiments were completed and the results are reported.

The design and use of a $3^{\text {rd }}$-generation friction-testing apparatus is presented. Previous research efforts used a constant-displacement control device to load the undeformed fabrics during the friction testing. While the information learned in these tests was valuable in developing a fundamental understanding of the tool-fabric behavior, the $3^{\text {rd }}$-generation friction-testing apparatus is a load-control device and yields a very complete fundamental understanding of the friction mechanisms by being able to study inter-layer friction between fabrics and tool/fabric friction on undeformed and deformed fabrics and to capture the static and dynamic friction behaviors of a woven-fabric system. Results are reported for two material systems - one has a thermoplastic matrix and the other has a thermoset matrix. 
The methodology has been implemented in the commercially available finite element programs ABAQUS and LSDYNA. The elements for the fabric models are inherent to these finite element programs, so no special coding of elements is required. The material and friction behaviors are incorporated in the finite element modeling by userdefined subroutines with simple input cards for specifying material and friction properties. Thus, the design tool is packaged in a form that makes the transition to industry relatively seamless and easily used by composite design engineers in industry.

The method has already been shown to be attractive to industry. During 2008-09, the UMass-Lowell research team collaborated with industrial colleagues at Ford Research Lab and General Motors to do forming simulations of a rear tub (trunk) and floor pan for the USCAR program with DOE funding. MAG-Industrial Automated Systems has expressed interest in collaborating with UMass-Lowell to use the simulation method to explore the forming of automotive parts using prepreg unidirectional tapes and woven-fabric composites with embedded optical fibers.

\subsection{The Thermostamping Manufacturing Process}

Thermostamping is a manufacturing process that can produce quality composite parts with processing costs and time margins that are comparable to the fabrication of the currently stamped metal parts. The thermostamping process deforms a flat sheet of machine-woven continuous fibers (glass and/or carbon) commingled with thermoplastic fibers into a particular geometry using a pair of dies.

The thermostamping process (Fig. 1) starts with the alignment of a sheet of fabric in a rigid steel frame. Multiple layers of fabric are stamped into one part to achieve the desired part thickness and mechanical properties. For a thermoplastic-matrix woven fabric, the frame and fabric are placed in an oven and are soaked until the fabric reaches a desired temperature for the thermoplastic polypropylene fibers, which are commingled with fiberglass fibers, to melt. The melting of the thermoplastic fibers infuses the fiberglass fibers with the resin. The frame is then aligned with a punch and die, and pressure is applied around the circumference of the punch area by binder plates. The application of force by the binder plate provides the in-plane forces that are necessary to reduce wrinkling of the fabric as the woven material is drawn into the die by the punch. The finished piece assumes the geometry of the die and punch and hardens into a firm part after the thermoplastic has cooled. For the thermoset material, the woven fabric is compounded with a pliable thermoset resin. The frame holding the woven fabric is stamped into shape using the punch and die and then held at elevated temperature to cure (harden) the thermoset resin matrix. A hemisphere formed from a commingled glass-polypropylene fabric using this thermostamping process is shown in Fig. 2. The primary mode of deformation for the fabric is rotation (shearing) of the yarns, also known as 'trellis deformation'.



Fig. 1 Schematic example of the thermostamping process. 


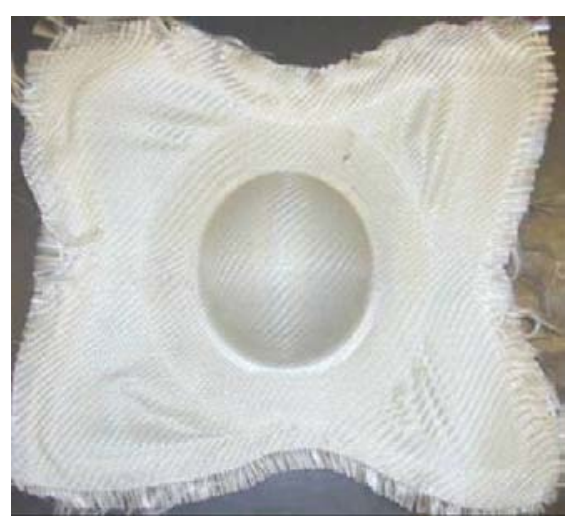

Fig. 2 Formed hemisphere using the thermostamping process.

The processing parameters associated with the thermostamping operation can have a significant effect on part quality. Fig. 3 shows the resulting part quality in a section under the binder plate from the stamping process of a commingled glass-polypropylene woven-fabric as studied by Wilks [1]. The resulting part quality is shown for cases of different pressure-and-temperature combinations. For this test, the pressure applied by the binder plate was controlled by springs and the binder plate was either unheated or heated during the process. With the binder plate unheated, fiber separation and tow breakage occurred sooner than when the binder plate was heated as pressure was increased (Fig. 3: d, f). The presence of fiber wrinkling was reduced as pressure increased (Fig. 3: e). Thus, it can be observed that part quality is a function of temperature and applied force, and in turn friction between the fabric and the tool.

The mechanical behavior of the woven fabric during the forming process is dominated by the evolution of the inplane shear stiffness as the yarns rotate and the uncrimping of the yarns due to in-plane tensile forces. By using a series of simple tests to characterize the mechanical behavior of a woven fabric, material parameters can be found. As an alternative to experimental characterization, there has been some limited success to develop purely analytical models of woven fabrics to estimate the mechanical behavior of a woven fabric. These analytically and/or experimentally determined material properties can be used in a finite element program to simulate the manufacturing process of a composite structure.

The inextensible characteristic of the yarns (fiberglass and/or carbon) requires draw-in and shearing of the fabric to occur for the material to conform to the tool geometry in forming the part. During fabric draw-in, the fabric slides under the metal binder plate and over the surfaces of the punch and die. For multilayer parts, there is possibly going to be some sliding between adjacent layers of fabric. Considering the interactions that take place between the fabric and tool and between adjacent layers of fabric during the manufacturing process and the effect of the process parameters, e.g. pressure, temperature and rate, on part quality, it is clear that characterizing the friction between the tool and the fabric and the friction between adjacent layers of fabric is critical to modeling the manufacturing process accurately.

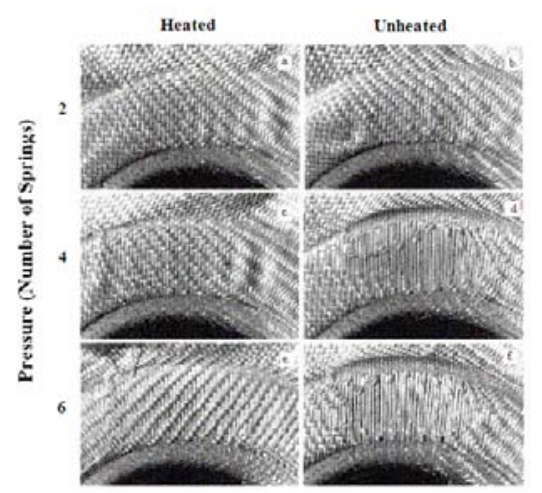

Fig. 3 Effect of temperature and pressure on part quality during the thermostamping process [1]. 


\subsection{Analytical Modeling of the Shear Behavior of Woven-Fabric Composites}

Trellis shear, as shown in Fig. 4, is the main deformation mode during the stamping process of woven-fabric composites [2]. Thus, the formability of woven-fabric composites is primarily a function of the shear properties of the woven fabric. Therefore, analytical models to obtain the shear properties of woven-fabric composites would be very useful to characterize the mechanical behavior of an existing fabric without doing time-consuming experiments or for characterizing a yet to made fabric for which experimentation is not an option.
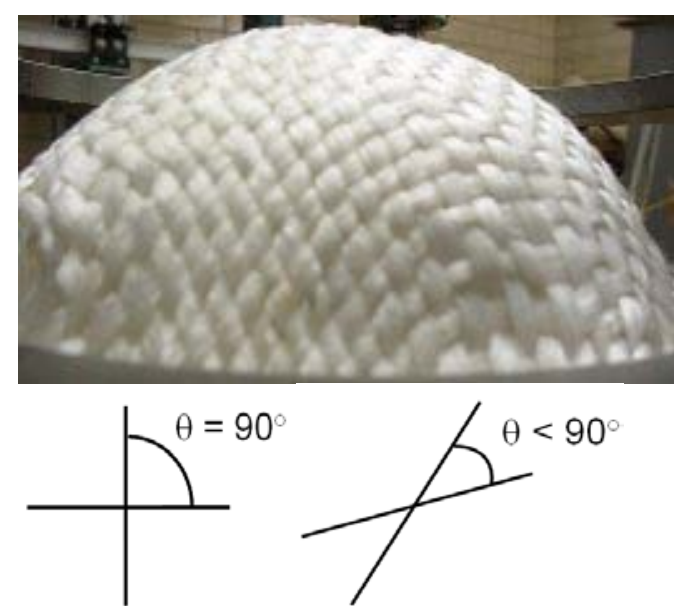

Fig. 4 Trellis deformation of a stamped hemisphere.

An analytical unit-cell model, which includes (1) the key shear-deformation-resistant mechanisms, (2) the friction between the warp and weft yarns at every crossover, and (3) the lateral compaction between adjacent yarns, was developed by Liu et al. $[3,4]$ to predict the shear properties of woven fabrics for the thermostamping process. In the model, some yarn parameters such as the yarn-to-yarn coefficient of friction $\mu$, the fiber contact frequency ratio $\beta$, and the ideal maximum fiber volume fraction $V_{a}$, were chosen to develop an empirical model to fit the experimental data. Validation of those parameters is necessary to complete the unit-cell model.

The maximum yarn-fiber volume fraction $V_{a}$ is an ideal limit to which fibers in a yarn can be compacted. As the yarn-fiber volume fraction approaches this maximum value, the stiffness in the transverse direction increases dramatically to approach the stiffness of the solid fiber material. According to the packing theory of uniform sizes of fibers, square packing corresponds to $V_{a}$ of $\pi / 4$ (or 0.785), and hexagonal packing results in a value of $\sqrt{3} \pi / 6$ (or 0.907). Because the diameters of the polypropylene and glass fibers are different in the commingled polypropylene/glass yarn, the square packing theory is modified for the commingled fibers, and the obtained $V_{a}$ equals 0.813 [5].

The compaction was studied using plane-strain compression tests, where 50-mm long fiber bundles were placed in a channel fixture as shown in Fig. 5. A compressive force was applied perpendicular to the axial direction of the fibers ( $Z$ direction) using an Instron machine at a rate of $0.042 \mathrm{~m} / \mathrm{sec}$. Load and extension data were logged so that stress and strain in the $Z$ direction could be calculated. Because the transverse compliance can also be calculated in the fiber bundle model as a function of $\beta$, regression of the experimental data will give the mean value of $\beta$. In Fig. 6, the predicted and experimental values of the yarn transverse compliance $S_{22}$ are compared for various values of $\beta$. The experimental results were obtained from the plane-strain compression tests for five layers of yarns as described in [6]. From Fig. 6, it can be seen that the values of $\beta$ are in the range from 150 to 175 for the commingled polypropylene and glass yarns explored in this research.

To investigate the sensitivity of the model to the yarn input parameters such as the maximum fiber volume fraction $V_{a}$, the fiber contact ratio $\beta$ and the yarn-to-yarn coefficient of friction $\mu$, a parametric study was conducted. To separate the individual contributions from friction and lateral compaction, intermediate models were studied separately, i.e., the lateral compaction resistant moment was set to be zero to study the coefficient of friction, while in the study of $\beta$ and $V_{a}$, the friction resistant moment was set to be zero in the equilibrium equation. 
Fig. 7 shows the results of the simulation of $\beta$ varying from 150 to 175 . In the figure, it can be seen that as the value of $\beta$ decreases, the shear load increases. The loads increase because at relatively low values of $\beta$, the yarn stiffness in the transverse direction increases due to increased fiber bending. As shown in Fig. 8, decreasing the value of $V_{a}$ has a similar effect because, with relatively low values of $V_{a}$, yarn stiffness in the transverse direction increases due to decreased space for compaction. Thus, with the main parameters all validated, the developed analytical model can be used to predict the shear properties of woven-fabric composites and reduce or eliminate the need for curve fitting of fabric-level experimental data.
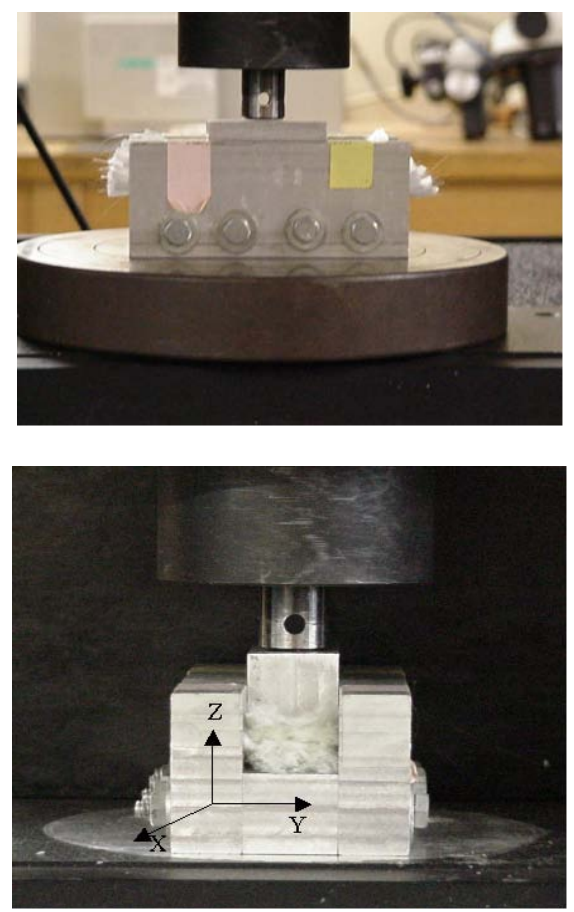

Fig. 5 Plane-strain compression test fixture [5].

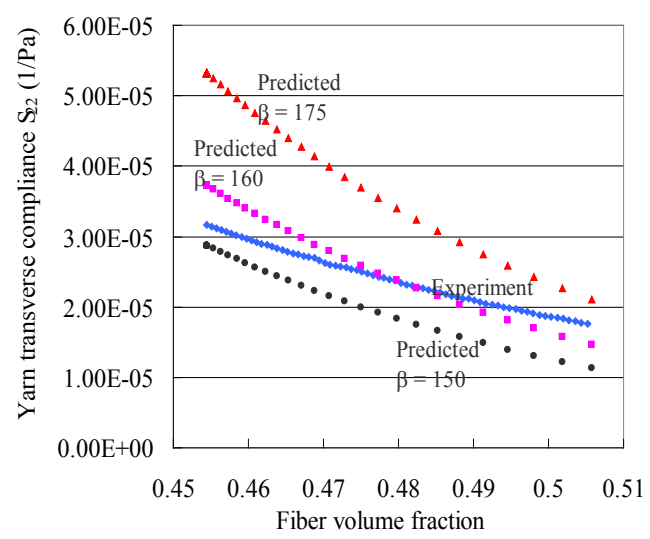

Fig. 6 Comparison of the values of $S_{22}$ obtained from the plane-strain compression tests and the fiber bundle modeling [6]. 


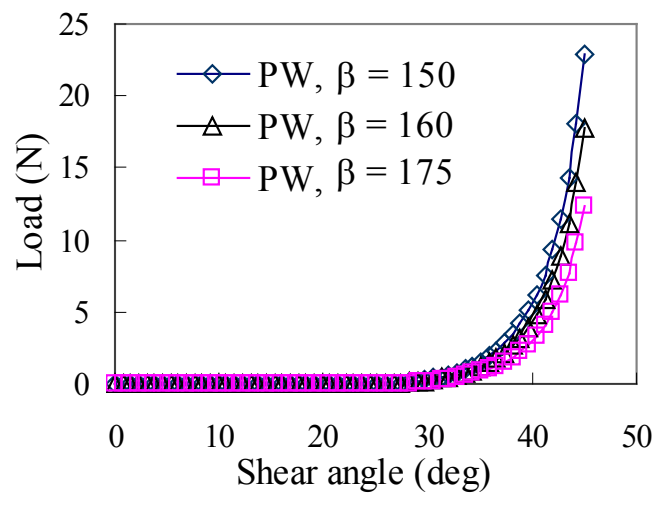

Fig. 7 Simulation of the fiber contact frequency ratio.

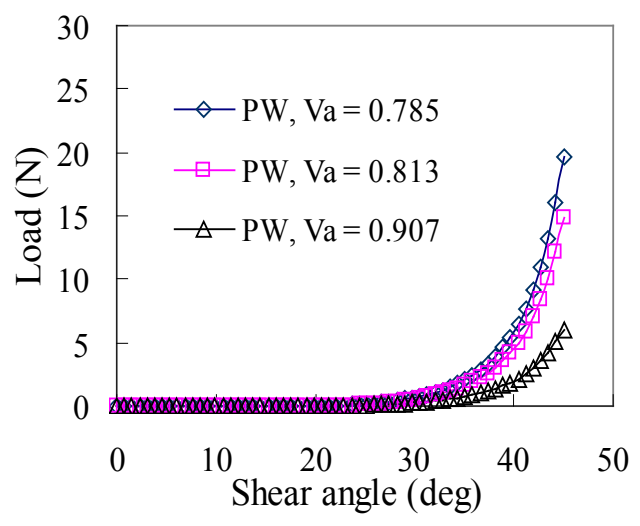

Fig. 8 Simulation of the maximum fiber volume fraction.

\subsection{Friction Behavior for Woven Fabrics}

Past NSF-sponsored research led to advances in a user-defined friction subroutine for use in finite-element applications. The Stribeck curve (Fig. 9 [7]) and the Hersey number, H, (Eq. 1) were used to find the friction coefficient for the ranges of velocities and normal forces studied.

$$
H=\frac{\eta \cdot U}{N}
$$

where $\eta$ is the viscosity, $U$ is the fabric velocity and $N$ is the normal force.

The results of parametric studies conducted by Gorczyca [8,9] showed that the velocity of the fabric, tool temperature, and applied normal force had the greatest effects on the friction coefficient. From these results, she developed a model that related the friction coefficient $\mu$ to the Hersey number,

$$
\mu=(6.1191 \cdot H+0.2718)-\mu_{v}
$$

where $\mu_{\nu}$ is a scaling term included for the effects of the tool temperature. Because the method of normal force application used in her research was displacement controlled, her results were limited to measuring only the static coefficient of friction $[8,10,11]$.

It was found that during a typical friction test the normal force applied to the fabric surface decreased, as shown in Fig. 10 where the initial applied load was approximately $1500 \mathrm{~N}$ and dropped by $\sim 400 \mathrm{~N}$ over the duration of the 
test. The mechanisms behind this decrease in normal force are fabric nesting and tow compaction. The fabric tows adjust their position as the adjacent layers of fabric nest, tow undulation decreases and fibers compact due to the combination of heat and pressure. Any one or more of these mechanisms causes a slight change in the displacement necessary to maintain the targeted normal force.

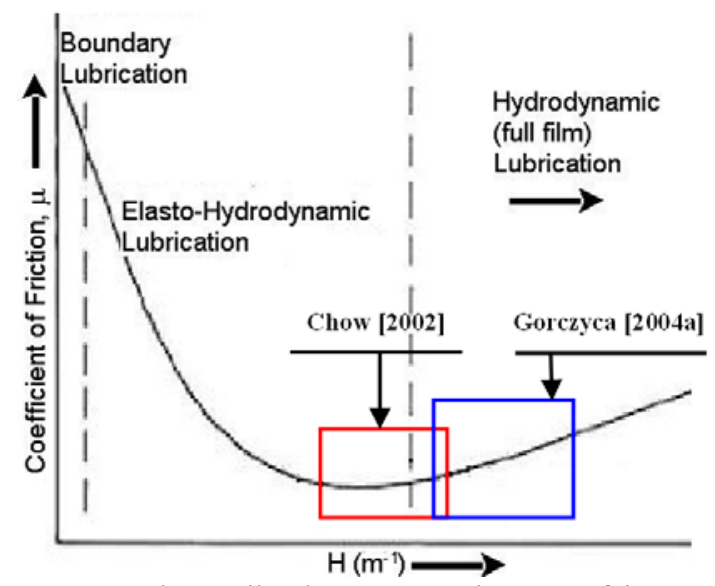

Fig. 9 The Stribeck Curve and areas of interest pertaining to current research. Adapted from [7].

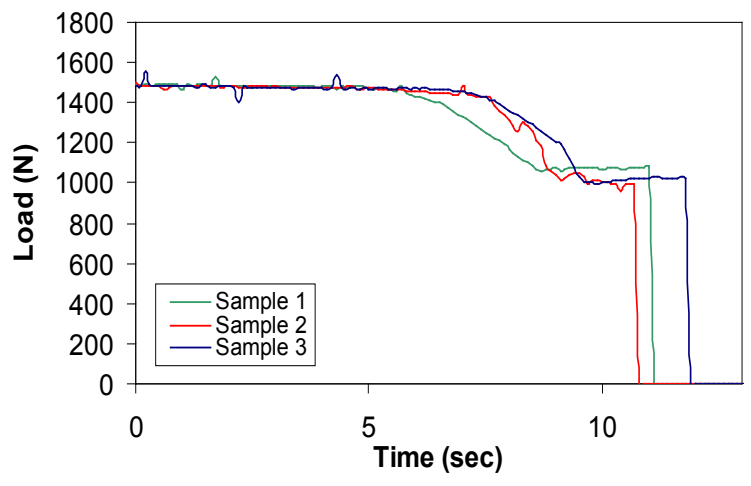

Fig. 10 Normal force decrease over time for displacement controlled test of commingled glass-polypropylene plain-weave fabric.

Due to the drop in normal force, only the static coefficient of friction, which is the value obtained at the first instant of fabric movement, could be reliably quantified by Gorczyca using the $2^{\text {nd }}$-generation displacement-control friction test apparatus. Being able to capture the dynamic coefficient of friction would give a friction model that accurately represents the frictional behavior over the entire duration of the thermostamping process where the friction is both static and dynamic.

To gain the ability to determine the dynamic coefficient of friction, a load-controlled application mechanism was designed and built as one component of the current research [12]. The force-controlled mechanism consists of a pneumatic air-spring actuator and a closed-loop control algorithm to continuously monitor and regulate the magnitude of force applied to the fabric throughout the friction test. The temperature of the tool and the pullout speed can be prescribed over a range of values that are observed to be present in the thermostamping process.

For the stamping of a hemisphere with a plain-weave fabric (as shown in Figs. 1 and 2), the tows are initially oriented $\sim 90^{\circ}$ with respect to one another, and this angle decreases as the fabric conforms to the dual-curvature surface of the hemisphere as shown in Fig. 4. 


\subsection{Friction-Testing Apparatus}

The $3^{\text {rd }}$-generation friction-testing apparatus is a stand-alone test machine. The limiting factor for the previous $1^{\text {st }}$ and $2^{\text {nd }}$-generation test apparatuses was their dependence on the Instron testing machine. By integrating all fabric movement into one test apparatus, the travel time from the oven to the press can be reduced from $\sim 20$ seconds to be less than 3 seconds, thereby reducing the influence of any cooling effects as the fabric is transferred from the oven to the press. Fig. 11 shows a schematic of the overall $3^{\text {rd }}$-generation friction-testing system, and Fig. 12 is a photograph of the press portion of the system.

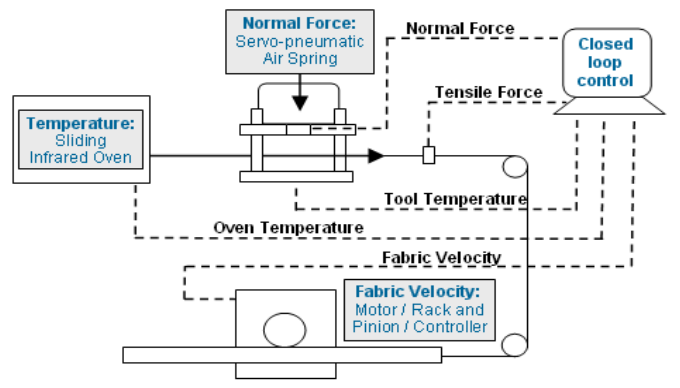

Fig. 11 Schematic representation of the $3^{\text {rd }}$-generation friction test apparatus.

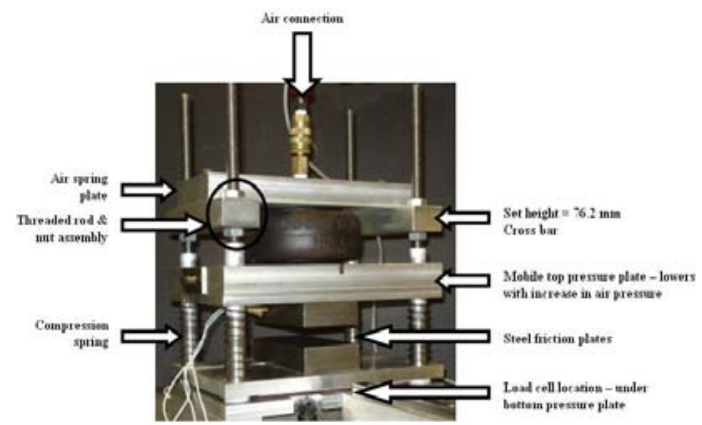

Fig. 12 The friction press.

\subsection{Friction Experiments}

The $3^{\text {rd }}$-generation load-control friction test apparatus [13] was used to capture the static and dynamic coefficients of friction. The objective of the friction-test apparatus was to accurately simulate the combinations of pressure, temperature and speed observed in the actual thermostamping process. In a friction test, the fabric is clamped in a holder (Figure 13(a)), heated in an infrared oven and subsequently shuttled to the press (similar to what is shown in Fig. 1 for the thermostamping process), which features two heated platens. A DC motor drives a rack and pinion to pull the sample through the press. The transfer from the oven to the press is done manually, and the fabric sample typically sits pressurized between the platens for about one second before the motor begins to pull. Experiments have shown that this one-second time is sufficient to keep the thermoplastic melted. 


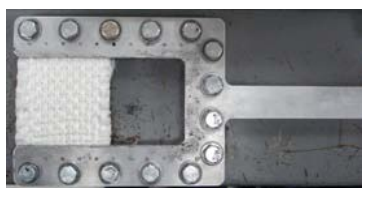

(a)

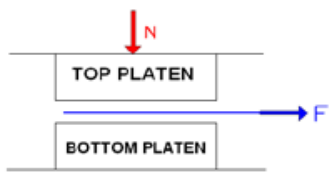

(b)

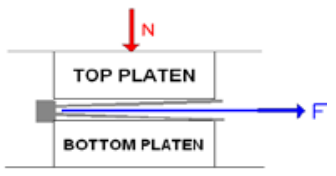

(c)

Fig. 13 (a) Fabric holder and schematics of the friction test setups for

(b) tool/fabric and

(c) fabric/fabric interfaces

For the fabric/fabric friction characterization, one fabric layer is placed between two "outside" layers of fabric. The two outer layers are clamped together on one end, and the inner layer is pulled between these two outer layers. The fully compressed platens prohibit the clamp which holds the two outer layers from moving. The fabric holder which is used to pull the fabric through the press is shown in Fig. 13(a) and schematics of the friction test setup for the fabric/tool and fabric/fabric conditions are shown in Figs. 13(b) and (c), respectively.

The effective coefficient of friction is calculated by

$$
\mu_{e f f}=\frac{F}{2 N}
$$

where the normal force, $N$, is multiplied by a factor of two to account for the two contacting surfaces on either side of the fabric sample.

The test conditions were also based on simulating a range of thermostamping process conditions. Experiments were performed on a Twintex ${ }^{\circledR}$ balanced plain-weave fabric. All samples were cut to be $51 \times 76 \mathrm{~mm}^{2}$, and tests were performed in triplicate to account for any variation among fabric samples.

The coefficient of friction was plotted against the pullout displacement. These curves can result in an initial peak, followed by a drop and then a relatively flat response for the Twintex ${ }^{\circledR}$ woven fabric used in this research. The peak and "flat" portions of the curves correspond to the static and dynamic coefficients of friction, respectively. The magnitude of the peak is a function of the pulling speed and pressure. Fig. 14 shows typical coefficient of friction vs. displacement curves for three tests at a pressure of $110 \mathrm{kPa}$, pullout velocity of $16.7 \mathrm{~mm} / \mathrm{s}$, and tool temperature of $85^{\circ} \mathrm{C}$.

A constant fabric area remains between the platens for approximately $50 \mathrm{~mm}$ of displacement. A drop in the fabric temperature after the fabric is in contact with the platens explains why small variations in the pullout force in the displacement range associated with the dynamic coefficient of friction are observed. 


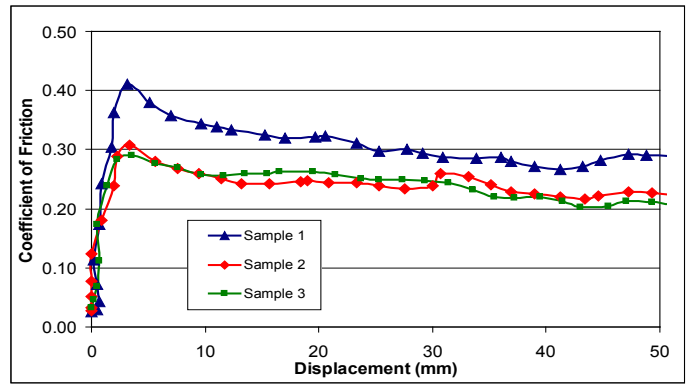

Fig. 14 Coefficient of friction as a function of pullout length for three tests at a pressure of $110 \mathrm{kPa}$, pullout velocity of $16.7 \mathrm{~mm} / \mathrm{s}$, and tool temperature of $85^{\circ} \mathrm{C}$.

\subsection{Friction Test Results}

Fig. 15 shows the static and dynamic coefficients of friction at the fabric/tool interface for the Twintex ${ }^{\circledR}$ balanced plain-weave fabric as a function of pullout velocity [14]. All tests were run with the baseline pressure of $387 \mathrm{kPa}$ and a tool temperature of $85^{\circ} \mathrm{C}$. The baseline values were determined from the typical processing conditions for the Twintex ${ }^{\circledR}$ fabric being considered in this research. In Stribeck theory, an increase in velocity causes an increase in the Hersey number. Similarly, an increasing velocity causes viscosity to increase, which also increases the Hersey number. In the hydrodynamic range of the Stribeck curve, an increasing Hersey number corresponds to an increasing coefficient of friction. Fig. 15 implies that the friction response is in the hydrodynamic behavior region of the Stribeck curve (Fig. 9) because the coefficient of friction increases with increasing velocity and likewise increasing Hersey number. Quantifying the variation of the friction coefficient as a function of velocity is important because during the forming process, the fabric does not necessarily move at a constant velocity along the tooling. In addition, as the fabric conforms to the shape of the die, the tows of the fabric are sheared and reoriented, affecting the rate at which the fabric slides over the surfaces that it is contacting.

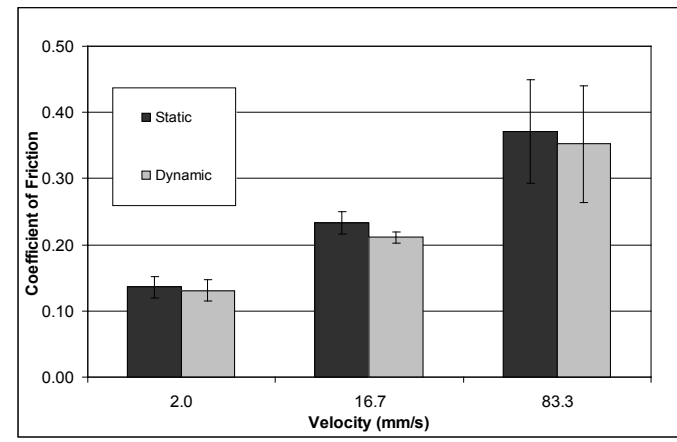

Fig. 15 Experimental results (fabric/tool) at different pullout velocities with the baseline pressure of $387 \mathrm{kPa}$ and the baseline tool temperature $85^{\circ} \mathrm{C}[14]$.

Fig. 16 shows the variation of the static and dynamic coefficients of friction with changes in normal force (or pressure). The pullout speed was the baseline value of $16.7 \mathrm{~mm} / \mathrm{s}$. In Eqn. (1), it can be seen that normal force is inversely related to the Hersey number. Therefore, the decrease in the friction coefficient with an increase in normal load shown in Fig. 16 also implies the friction behavior to be in the hydrodynamic-friction region of the Stribeck curve (Fig. 9). 


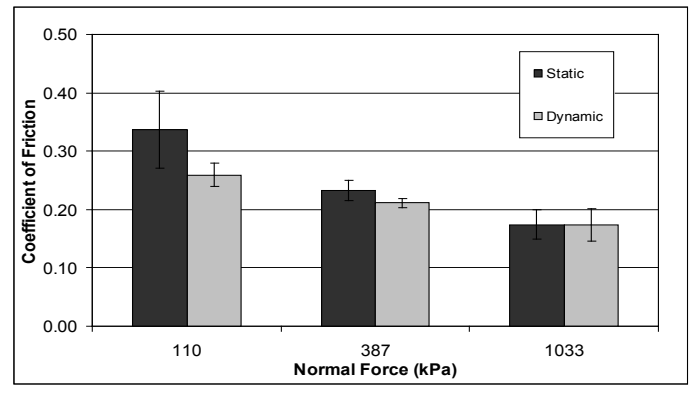

Fig. 16 Experimental results (fabric/tool) at different normal loads with the baseline pullout velocity of $16.7 \mathrm{~mm} / \mathrm{s}$ and the baseline tool temperature of $85^{\circ} \mathrm{C}[14]$.

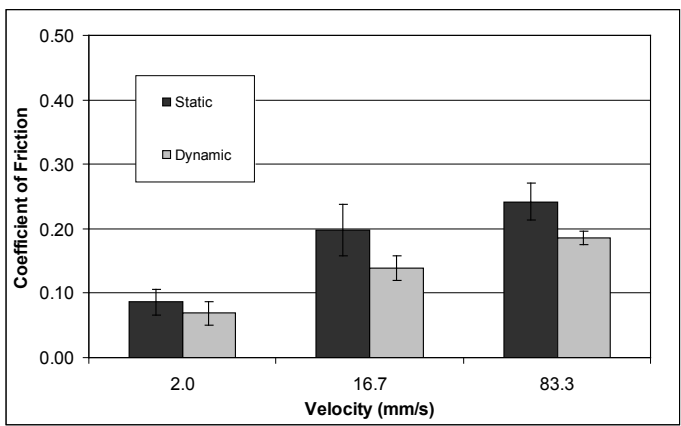

Fig. 17 Experimental results (fabric/fabric) at different pullout velocities with the baseline pressure of $387 \mathrm{kPa}$ and the baseline tool temperature of $85^{\circ} \mathrm{C}$ [14].

Fig. 17 shows the static and dynamic coefficients of friction for the balanced plain-weave fabric at the fabric/fabric interface as a function of pullout velocity. Similar to the fabric/tool interface, the coefficient of friction was observed to increase with increasing velocity at the fabric/fabric interface.

Fig. 18 displays the effect of normal force on the friction coefficient at the fabric/fabric interface. The decrease in the coefficient of friction with increasing normal force follows the same trend as the results from the fabric/tool interface. Similar trends for the coefficients of friction as a function of normal force and velocity were observed at both the fabric/tool and fabric/fabric interfaces, where only the magnitude of the friction coefficients differed. These trends support that the Stribeck curve can explain the observed friction behaviors.

Due to significant differences in friction coefficients under different processing conditions, any finite element simulation of the manufacturing process must be able to capture the coefficient of friction as a function of pressure, velocity and temperature. Such dependencies have been implemented in ABAQUS/Standard [8] ABAQUS/Explicit, and LS-DYNA (explicit version) $[15,16]$ through user-defined friction subroutines for finite element modeling of the thermostamping process.

Friction testing was completed to study the effect of fabric orienation and the state of shear. For tool/fabric and fabric/fabric frictions, the the experimental data implied that these paramters were essentially negligle on the resulting friction coefficients. 


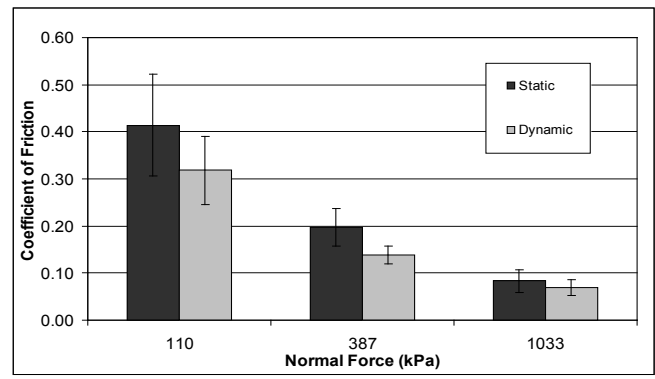

Fig. 18 Experimental results (fabric/fabric) at different normal loads with the baseline pullout velocity of $16.7 \mathrm{~mm} / \mathrm{s}$ [14].

\subsection{Characterization of the Mechanical Behavior of the Woven Fabrics using Experimental Methods}

Experimental data were used to derive the material parameters for the constitutive models used in the simulation of the mechanical behavior of the fabrics studied in the forming simulations of the current research. For the forming of composite parts using woven fabrics, these material parameters were needed to describe the nonlinear tensile behavior of the yarns and the nonlinear shear behavior of the fabric.

A yarn behaves differently when incorporated within a crimped fabric than alone because of the undulations of the yarns and the influence of the perpendicular yarns in the fabric. A biaxial tensile test allows the stretching of the fabric in the two yarn directions at the same time, and hence, the determination of the tensile properties of the fabric due to a variety of in-plane-force combinations. In a first approximation, models generally do not account for the effect of the tension in the lateral direction, and that is why the simpler uniaxial testing of single yarns was chosen for the material characterization in this research.

The nonlinear tensile behavior of the fabric due to the decrimping is essential to perform reliable simulations [17]. If the crimp ratio (difference between the length of the yarn and the length of the fabric) is known, then the decrimping part of the stress-strain curve can be extrapolated from the experimental data.

The in-plane shear properties were obtained by a shear-frame test. In the speed range attainable with a conventional tensile machine, the fabric load-deformation response in the shear-frame test was not found to be rate dependent [3].

\subsection{Experimental Results of the Mechanical Behavior}

Three Twintex ${ }^{\circledR}$ woven fabrics composed of commingled polypropylene and glass fibers designed for thermostamping were tested [18]. The fabric characteristics can be found in [19]. PW, TWI and TWII refer to the plain-weave; the thin-twill-weave and the thick-twill-weave fabrics, respectively.

Uniaxial tensile testing was performed on an Instron 4464 machine with a 2-kN load cell. Pneumatic cord and yarn grips were used, and the gauge length was set to approximately $1 \mathrm{~m}$ to minimize the effect of the deformation in the grips. The displacement rate was $5 \mathrm{~mm} / \mathrm{s}$.

The stiffness of the yarns was determined from the slope of the load/true-strain curves. In addition, measurements of the crimp ratio were performed. The results, presented in Table 1, were used to assess the tensile behavior of the yarn within the fabric via an extrapolation of the decrimping (Fig. 19).

Shear-frame tests were performed on the Instron 4464 machine. The geometry chosen for the specimen is shown in Fig. 20. Note that to avoid the shearing contribution of the arms, the cross yarns were removed from this part of the sample. The length of the frame $L_{F}$ was $216 \mathrm{~mm}$, and the length of the fabric $L_{f}$ was $120 \mathrm{~mm}$. 
Table 1: Yarn tensile stiffness [18]

\begin{tabular}{lll}
\hline Pattern & $\begin{array}{l}\text { Tensile stiffness } \\
(\mathrm{kN})\end{array}$ & $\begin{array}{l}\text { Crimp } \\
\text { ratio (\%) }\end{array}$ \\
\hline PW & $24.6 \pm 0.7$ & 2.0 \\
TWI (warp/weft) & $25.1 \pm 0.6 /$ & $4.4 / 0.8$ \\
TWII (warp/weft) & $50.3 \pm 0.4$ & $3.0 / 0.0$ \\
\hline
\end{tabular}

The specimen was sheared at the rate of $2 \mathrm{~mm} / \mathrm{s}$. A mechanical conditioning of five pre-test shearing runs was applied. The mechanical conditioning ensured a uniform distribution of the yarns across the sample. The normalized shear force $F_{s h}$ and the shear angle $\gamma$ were determined from the crosshead displacement $\delta$ and the total load on the frame $F$ by the following equations [19]:

$$
\begin{gathered}
\gamma=\frac{\pi}{2}-2 \cdot \arccos \left(\frac{\delta}{2 \cdot L_{F}}+\frac{\sqrt{2}}{2}\right) \\
F_{s h}=\frac{L_{F}}{L_{f}^{2}} \frac{F}{2 \cos (\pi / 4-\gamma / 2)}
\end{gathered}
$$

Tests were performed at room temperature and at $175^{\circ} \mathrm{C}$, which is the recommended processing temperature for forming of these fabrics. The results are presented in Figs. 21 and 22.

The TWI fabric has a very tight weave and exhibits logically higher shear stiffness than the two other Twintex ${ }^{\circledR}$ fabrics. It is worth noting that the repeatability (represented by the error bars) is good up to the locking angle $\left(\sim 30^{\circ}\right.$ for TWI, $\sim 40^{\circ}$ for PW and TWII). At least five tests were used to obtain a reliable average.

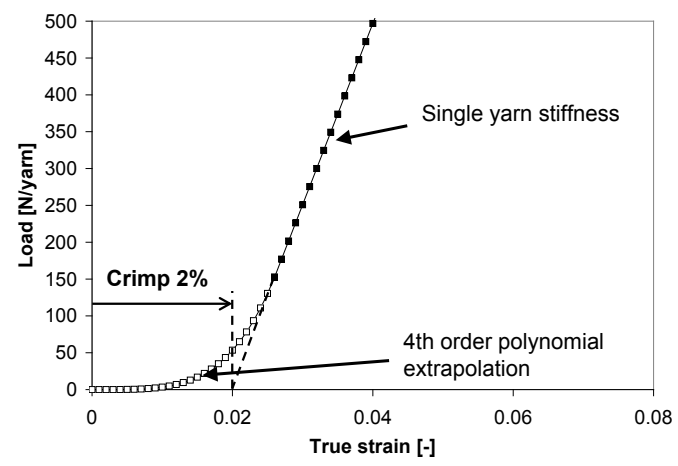

Fig. 19 Extrapolation of the fabric tensile behavior from the single yarn stiffness and the crimp ratio $(\mathrm{PW})$. 


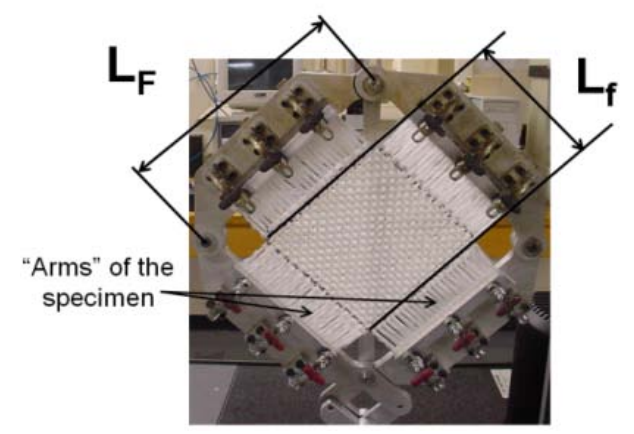

Fig. 20 Geometry of the shear frame. Free yarns have been removed in the "arms" of the specimen.

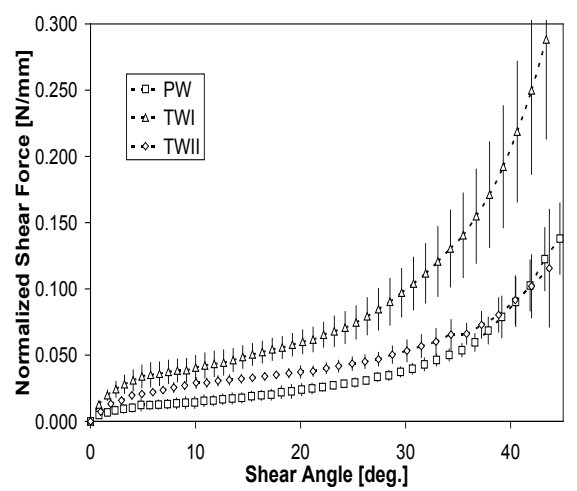

Fig. 21 In-plane shear behavior at room temperature [18].



Fig. 22 In-plane shear behaviour at $175^{\circ} \mathrm{C}[18]$.

\subsection{Finite Element Modeling [20,21]}

Several modeling approaches have been developed to account for the evolution of the orthotropic directions during high shearing, and these approaches include the continuous approach, the semi-discrete finite element approach and the mesoscopic approach [22]. The first two approaches, while giving good results and being efficient in terms of computing time are generally somewhat complex and sometimes very challenging to implement into commercially available FEA packages. In the current research, a discrete mesoscopic approach, based on built-in elements and user-defined material and friction subroutines in commercially-available finite element codes was used. The explicit codes used were ABAQUS and LS-DYNA, and the material parameters were derived from conventional tensile and shear-frame experimental data as previously discussed in this report. 
The method was also implemented into ABAQUS/Standard (implicit solver) [23]. However, problems with convergence in ABAQUS/Standard due to the contact of the fabric with binders other than binder plates that covered all of the fabric could not be overcome. The elements needed for the methodology are not currently available in LS-DYNA implicit solver, so using the LS-DYNA implicit solver for the process simulation was not an option for this research.

\subsection{Finite Element Model Description}

The unit cell of the mesoscopic model used in this research for a plain-weave fabric consists of four 1-D elements and one 2-D element as shown in Fig. 23 for a plain-weave fabric. The 1-D elements carry the tensile loads through the fabric and are able to rotate freely as the fabric is sheared. This rotation of the 1-D elements is a simple and automatic way of accounting for the local changes in orientation of the principal load paths through the fabric. The 2-D element is included in the model to capture the in-plane shear stiffness of the fabric and has no tensile stiffness. The respective material models for the 1-D and 2-D elements were implemented in the finite element codes by userdefined subroutines. These material models capture the nonlinear load-displacement behavior of the fabric for inplane tensile and shearing deformations. Unit cells can likewise be defined by a combination of 1-D and 2-D elements for other weave patterns, such as satin and twill weaves [21,23].

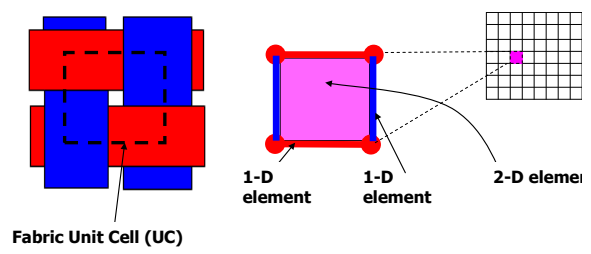

Fig. 23 Representative unit cell of the hybrid mesoscopic modeling of a plainweave fabric [23].

\subsection{Constitutive Equation for 1-D Elements}

The explicit finite element codes ABAQUS and LS-DYNA allow for customized stress-strain relationships at each time step via a user-defined material subroutine [24]:

$$
\Delta \sigma_{i j}^{t+1}=C_{i j k l} \Delta \varepsilon_{k l}^{t+1 / 2}
$$

where $\Delta \sigma_{i j}^{t+1}$ is the stress increment at time step $t+1, C_{i j k l}$ is the constitutive matrix defined by the user-supplied material parameters at time step $t$, and $\Delta \varepsilon_{k l}^{t+1 / 2}$ is the midpoint strain increment from the solver. For 1-D elements, Eq. (6) becomes:

$$
\Delta \sigma_{11}^{t+1}=C_{11}\left(\varepsilon_{11}\right) \cdot \Delta \varepsilon_{11}^{t+1 / 2}
$$

where $C_{11}$ is the tangent tensile modulus and is a function of $\varepsilon_{1 I}$. This tangent modulus is easily deduced from experimental tensile stress-true strain data of the fabric.

\subsection{Constitutive Equation for 2-D Elements}

The incorporation of the in-plane shear behavior in a shell/membrane element for finite-strain analysis is more challenging than the tensile behavior of the 1-D elements. Two reference frames as depicted in Fig. 24 have to be considered as the fabric shears from its initial orthogonal configuration to a nonorthogonal configuration. The $\boldsymbol{e}_{\boldsymbol{i}}$ unit vectors define the local orthogonal reference frame that rotates with the material, and the $\boldsymbol{g}_{\boldsymbol{i}}$ basis vectors form a nonorthogonal frame that follows the fiber directions: 


$$
\vec{g}_{i}=\underline{\underline{F}} \cdot \vec{g}_{i}^{0}=\underline{\underline{F}} \cdot \vec{e}_{i}^{0}
$$

where $\underline{\underline{F}}$ is the deformation gradient tensor known at each increment in the deformation process.

The in-plane shear-stress/shear-strain behavior of the woven fabric is captured by:

$$
\begin{aligned}
& \Delta \widetilde{\sigma}_{12}^{t+1}=\widetilde{C}_{33}\left(\gamma_{12}\right) \cdot \Delta \gamma_{12}^{t+1 / 2} \\
& \text { with } \Delta \gamma_{12}^{t+1 / 2}=2 \cdot \Delta \varepsilon_{12}^{t+1 / 2}
\end{aligned}
$$

where $\Delta \widetilde{\sigma}_{12}^{t+1}$ is the shear-stress increment expressed in $\boldsymbol{g}_{i}$ and $\widetilde{C}_{33}\left(\gamma_{12}\right)$ is the tangent shear modulus that can be determined from experimental data or by using the analytical method described earlier in this paper. This constitutive equation uses the tensorial shear-strain increment $\Delta \varepsilon_{12}^{t+1 / 2}$ expressed in the $\boldsymbol{e}_{\boldsymbol{i}}$ reference frame as it is given by the finite element solver to the user-defined material subroutine. However, the obtained stress increment is expressed in terms of the $\boldsymbol{g}_{\boldsymbol{i}}$ basis and needs to be returned to the finite element solver in the $\boldsymbol{e}_{\boldsymbol{i}}$ basis at the end of the user-defined subroutine. The rotation matrix relating the two reference frames can be obtained from a geometric analysis [25]. The 1-D elements carry the stresses in the $\boldsymbol{g}_{1}$ and $\boldsymbol{g}_{2}$ directions, and consequently $\Delta \widetilde{\sigma}_{11}=\Delta \widetilde{\sigma}_{22}=0$ in the $\boldsymbol{g}_{\boldsymbol{i}}$ reference frame within the 2-D element. Therefore, the stress increments are returned to the finite element solver in the $\boldsymbol{e}_{\boldsymbol{i}}$ basis using:

$$
\begin{aligned}
& \Delta \sigma_{11}=2 \cdot \cos \alpha \cos (\alpha+\theta) \cdot \Delta \widetilde{\sigma}_{12} \\
& \Delta \sigma_{22}=2 \cdot \sin \alpha \sin (\alpha+\theta) \cdot \Delta \widetilde{\sigma}_{12} \\
& \Delta \sigma_{12}=\sin (2 \alpha+\theta) \cdot \Delta \widetilde{\sigma}_{12}
\end{aligned}
$$

where the $\Delta \sigma_{i j}$ are the stress increments in the $\boldsymbol{e}_{i}$ reference frame and the $\Delta \widetilde{\sigma}_{i j}$ are the stresses in the $\boldsymbol{g}_{i}$ reference frame. The angle between $\boldsymbol{e}_{1}$ and $\boldsymbol{g}_{1}$ is $\alpha$, and the angle between $\boldsymbol{g}_{1}$ and $\boldsymbol{g}_{2}$ is $\theta$.

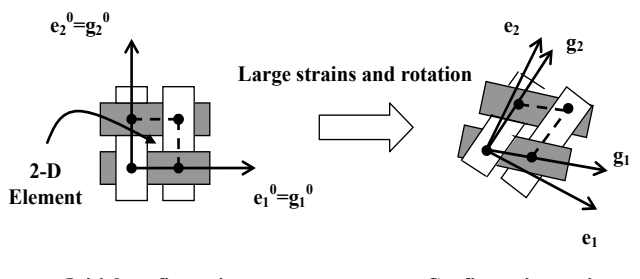

Fig. 24 Schematic representation of the reference frames used.

To be consistent with finite element codes that use the logarithmic strains for the summation of the strains increments, the logarithmic definition of strains should be used to determine $\widetilde{C}_{33}\left(\gamma_{12}\right)$ from the experimental data or analytical shear-frame model of the fabric. The true or "logarithmic" shear angle $\gamma^{L}$ can be obtained from the logarithmic strains in the principal stretch directions $\varepsilon_{I}^{L}$ and $\varepsilon_{I I}^{L}$ by a $45^{\circ}$ Mohr's circle transformation. In the case of a shear frame test, a geometrical analysis leads to the expression of $\gamma^{L}$ as a function of the geometric shear angle $\gamma:$

$$
\gamma^{L}=\varepsilon_{I}^{L}-\varepsilon_{I I}^{L}=-\ln (\tan (\pi / 4-\gamma / 2))
$$


The shear stress $\widetilde{\sigma}_{12}$ is equal to the normalized shear force $F_{s h}[18]$ divided by the thickness of the fabric. Finally, $\widetilde{C}_{33}\left(\gamma^{L}\right)$ is obtained from a derivation of $\widetilde{\sigma}_{12}$ versus $\gamma^{L}$ of the experimental shear frame test data or using the analytical shear-frame model.

\subsection{Implementation via the User-Defined Material Subroutines}

The method has been implemented via user-defined material subroutines in the explicit finite element codes ABAQUS and LS-DYNA. For ABAQUS, B31 beam elements and S4R shell elements were used, and for LSDYNA seatbelt elements and Hughs-Liu reduced integration shells (Shell 6) were used. Truss elements can also be used in either ABAQUS or LS-DYNA for modeling of the yarns. However, for large and complex deformations, the convergence of the analysis was found to be difficult to achieve when using truss elements. The LS-DYNA seatbelt element is very efficient in terms of computational time and has the added benefit of having zero compressive stiffness. The modeling of the shear contribution to the material stiffness could potentially be captured using either shell or membrane elements with careful attention being given to the accuracy of the element response at high shear strains.

To demonstrate the capabilities of the methodology to simulate the manufacturing process for Twintex ${ }^{\circledR}$ woven fabrics, plain-weave experimental data at room and elevated temperatures were used to conclude the material parameters for the user-defined material subroutines used in the finite element simulations. The shear stress versus $\gamma^{L}$ curve was first developed from normalized experimental data:

- $\quad$ shear stress was assessed from normalized shear force $F_{s h}$ assuming a fabric thickness of $0.5 \mathrm{~mm}$, and

- $\quad \gamma^{L}$ was obtained from the geometrical shear angle $\gamma$ using Eq.(11).

The tangent shear modulus $\widetilde{C}_{33}\left(\gamma^{L}\right)$ was then obtained by fitting a $4^{\text {th }}$-order polynomial to the shear stress vs. $\gamma^{L}$ experimental data.

Similarly the tangent tensile modulus was obtained from the tensile load vs. true strain experimental data. The "effective" section of the yarn $A_{\text {yarn }}$, with the assumption of no voids among the fibers was used. It was determined from the known data using the following equation [23]:

$$
A_{\text {yarn }}=\frac{\rho_{\text {yarn }}}{V F_{\text {glass }} \cdot \rho_{\text {glass }}+\left(1-V F_{\text {glass }}\right) \cdot \rho_{P P}}
$$

where $V F_{\text {glass }}$ is the glass volume fraction, $\rho_{\text {yarn }}$ is the yarn linear density, and $\rho_{\text {glass }}$ and $\rho_{P P}$ are the glass and polypropylene densities, respectively. The resulting $A_{\text {yarn }}$ is for the fabric being considered in the study. A square beam section was chosen for ABAQUS beams. However, other cross-section geometries (e.g., circle or oval) can be implemented should they be deemed more representative of the fabric. Because of the specific formulation of LSDYNA seatbelt elements, only the section area could be defined and no cross-section type could be described.

\subsection{Validation}

To validate the modeling approach, a shear frame test and a bias extension test were simulated at room temperature, and the results were compared to experimental data. A finite element model of the shear-frame test used at UMass Lowell was built, including the "arms" of the specimens [18]. The procedure used for completing the shear-frame testing required the removal of the free yarns in the arms of the specimens, so only 1-D elements were used in the model in the area of the arms (Fig. 25(a)). A bias-extension test was also simulated. The characteristic shear-strain field of this test [26] was obtained as shown in Fig. 25. 


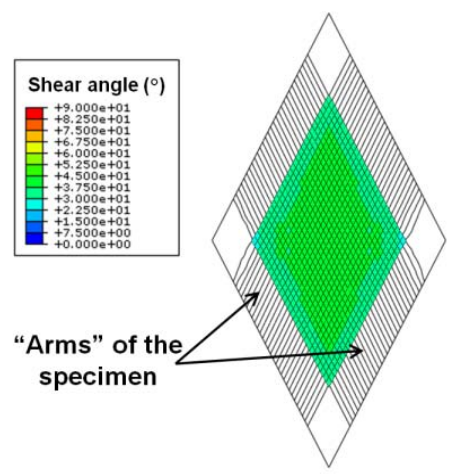

(a)

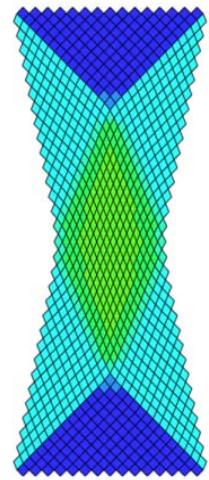

(b)

Fig. 25 Shear angle contour of (a) the shear frame and (b) the bias extension models (ABAQUS).

Figs. 26 and 27 show a comparison among finite element results from ABAQUS and LS-DYNA and experimental data to previous experimental work at UMass-Lowell (Shear frame data [18]) and to experimental data from Peng and Cao (Bias extension data [25]) for the Twintex ${ }^{\circledR}$ woven-fabric thermoplastic composite. Very good correlation was obtained between the ABAQUS and LS-DYNA results for both test conditions. The error bars in Fig. 26 denote one standard deviation. The simulations correlate well with the experimental data for the shear frame test, which validates the approach used, in particular the way the in-plane shear behavior is implemented. Concerning the biasextension test, experimental and simulation curves tend to diverge after $35 \mathrm{~mm}$ of displacement. This divergence could be a consequence of yarn sliding during the experiment [27] that is not considered in the simulation. This fact has been recently confirmed by experimental data obtained with a Digital Correlation Image technique used to track the shear deformation during the bias-extension test [21]. However, this sliding occurs at very large shear angles that may not be practical in the manufacture of quality parts using this composite forming process.

\subsection{Thermostamping Modeling}

The stamping of a hemisphere was simulated to demonstrate the capability of the approach for compound curvatures. A binder was used to induce in-plane tensile forces within the fabric to eliminate wrinkling. The two finite element codes give very similar results in terms of shear angle contours (Fig. 28). Although an actual thermostamped part is not shown here, these finite element results agree with what is observed in an actual part. For these simulations, the coefficient of friction was 0.3 .



Fig. 26 Comparison of the load-displacement curves for the experimental and finiteelement simulations of the shear-frame test. 


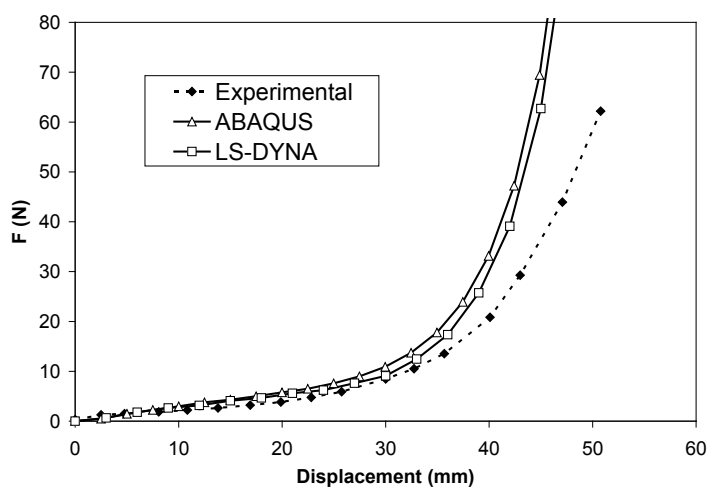

Fig. 27 Comparison of the load-displacement curves for the experimental and finiteelement simulations of the bias-extension test.



(a)
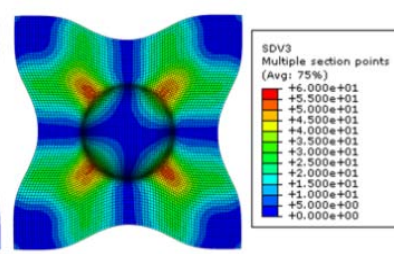

Fig. 28 Shear angle contour comparison between (a) LS-DYNA and (b) ABAQUS simulations.

\subsection{Industrial Application of the Design Tool}

The proposed simulation methodology was also applied to the forming of a composite tub for the automotive industry [28]. The fabric used for these simulations was a plain-weave fabric compounded with a thermoset resin which is pliable at room temperature. This fabric was characterized experimentally using tensile, shear-frame and friction tests. The friction was determined by the $3^{\text {rd }}$-generation friction testing apparatus to be essentially zero due to the lubrication effect resulting from the compounding material. The woven-fabric material properties were incorporated in the finite element model to simulate the forming process. The resulting parts are shown in Fig. 29.

For the tub shown in Fig. 29, the top contour plot shows the resulting shear-strain distribution across the part for a $0^{\circ} / 90^{\circ}$ ply orientation, and the bottom is for a $\pm 45^{\circ}$ ply orientation. The shear contours range from $0^{\circ}$ to $60^{\circ}$ as the color contours span from blue to red, respectively. Any shear angles above $60^{\circ}$ are shown in white. Note how the $0^{\circ} / 90^{\circ}$ ply is blue over a majority of the part. As a result, there is very high shearing in the corners to allow the fabric to assume the shape of the tub. This high shearing of greater than $60^{\circ}$ is associated with localized wrinkling. By rotating the fabric $45^{\circ}$ as shown in the bottom contour plot, the shearing can be distributed across the part and thereby avoid locally high shearing which can result in wrinkling. 

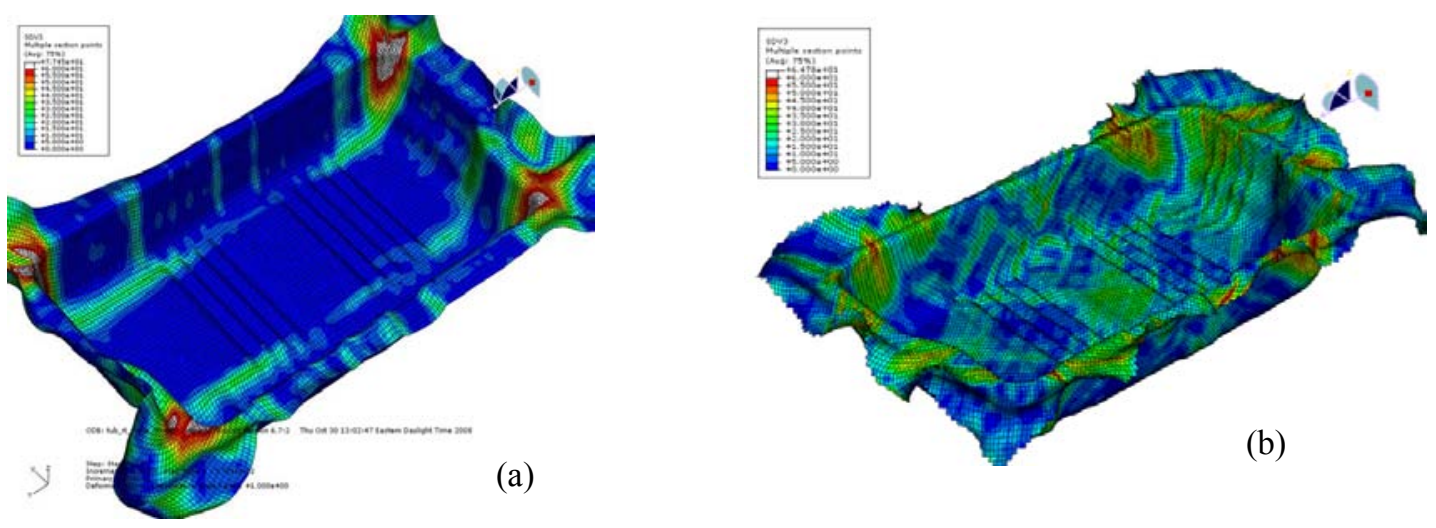

Fig. 29 Forming simulation of the Tub for a $0^{\circ} / 90^{\circ}$ ply orientation (a) and a $\pm 45^{\circ}$ (b). Shear angle contour (blue to red $=0^{\circ}$ to $60^{\circ}$, white above $60^{\circ}$ ).

This simulation of the tub forming process using the design tool developed in this NSF-funded research was completed as part of an ACC (Automotive Composites Consortium) research project for USCAR. The objectives of the simulations were to give insight as to problematic areas that may occur during the manufacturing process, i.e. wrinkling that could comprise the quality of the composite tub, and what processing steps may be taken to alleviate these problematic areas. In addition, by using the design tool to look at the forming of the tub using multiple layers of fabrics with varying orientations, the resulting structural stiffness of the composite tub can be accessed. Thus, the technological advances coming from this NSF research are being implemented by the US automotive industry to advance the use of composites in automotive designs leading to lighter and more fuel efficient cars and in reducing the cost of manufacturing composite parts.

Because of the success in applying the results of this composite forming simulation tool to the USCAR project, the UMass-Lowell team has been invited to collaborate with MAG-Industrial Automated Systems (formerly Cincinnati Machine) to explore the potential to form composite parts for the automotive industry using prepreg unidirectional tapes and woven fabrics. A joint proposal to NCMS (National Center for Manufacturing Science) is currently being written for submission for a January 2010 project start date. At the time of this report (May 2010), DOE had not yet confirmed or regretted the funding of the proposal.

\subsection{International Benchmarking Program}

In an effort to understand the strengths and limitations of the different simulation methods that are being developed by various research teams around the world, an international benchmark survey was conducted. Several groups submitted simulation results for the thermostamping of plain-weave Twintex fabric using a double-dome geometry as shown in Fig 30. Comparisons were made by observing the resulting draw-in of the fabric and shear angles developed in the fabric after stamping. As a result, a standard set of testing parameters was developed for a future round of simulations for comparison among the research groups.

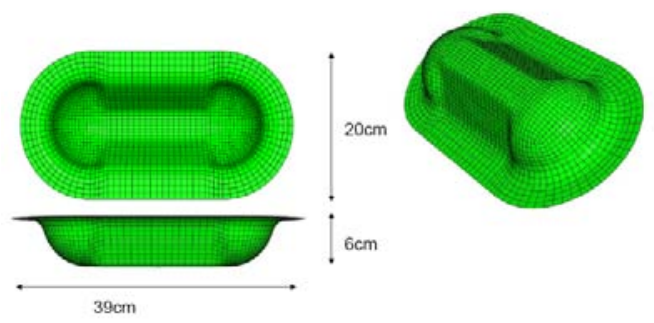

Fig. 30 Finite element mesh of the Double-Dome Geometry 


\subsection{Materials and Geometry}

The following bullets list the items to be used in the next round of simulations amongst the various international research teams:

- Double-dome tool (Fig. 30)

- Twintex fabrics (Plain and Fat-Twill weaves)

- Alternate fabrics tested and formed in participant labs

All simulation results will be compared to those of formed parts that were completed at Ford Research Labs in 2004 using the fat-twill fabric.

\subsection{Simulation Parameters}

After analyzing and comparing simulation results from several groups after a first round of simulations by the respective research groups, a uniform set of simulation parameters for study has been determined for use in a second round of simulations. These parameters include the orientation of the material blank, the amount of binder force applied and the type of material being simulated. The orientation of the fiber blank will be varied between the $0 / 90^{\circ}$ and $\pm 45^{\circ}$ orientations. The amount of binder force applied will be varied between a very low binder force (close to 0 ) and a large amount of force $(1000 \mathrm{~N})$. The materials being simulated will be characterized by two different sets of tensile and shear stress curves which will be provided to all groups based on the UMass-Lowell test data.

Other parameters defined in the simulations will be set at specific values for all groups. These are parameters that had less effect on the simulation results from Round 1 than the parameters previously discussed and include the coefficient of friction and the size of the material blank. The coefficient of friction will be set at 0.3 because no other team other than the UMass-Lowell is known to have the ability to account for variable friction. The material blank size will be set at $300 \mathrm{~mm}$ x $450 \mathrm{~mm}$. The parameters for each simulation are presented in Table 2 and the standard parameter values are summarized in Table 3. In Table 2, PW denotes plain weave and FT denotes fat twill weave.

Table 2: Simulation parameter matrix.

$\begin{array}{cccc}\text { Simulation } & \text { Mat'l } & \begin{array}{c}\text { Blank } \\ \text { Orientation }\end{array} & \begin{array}{c}\text { Binder } \\ \text { Force (N) }\end{array} \\ 1 & \text { PW } & 0 / 90^{\circ} & 0 \\ 2 & \text { PW } & 0 / 90^{\circ} & 1000 \\ 3 & \text { PW } & \pm 45^{\circ} & 0 \\ 4 & \text { PW } & \pm 45^{\circ} & 1000 \\ 5 & \text { FT } & 0 / 90^{\circ} & 0 \\ 6 & \text { FT } & 0 / 90^{\circ} & 1000 \\ 7 & \text { FT } & \pm 45^{\circ} & 0 \\ 8 & \text { FT } & \pm 45^{\circ} & 1000\end{array}$

Table 3: Constant simulation parameters.

$\begin{array}{ccc}\text { Parameter } & \begin{array}{c}\text { Coefficient } \\ \text { of Friction }\end{array} & \begin{array}{c}\text { Blank Size } \\ (\mathrm{mm})\end{array} \\ \text { Value } & 0.3 & 300 \times 450\end{array}$

\subsection{Comparison Metrics}

Comparison of simulation results will rely on the same metrics used in the first round of comparisons. These include the amount of fabric draw-in at three specified points as well as the shear angles reported along the apex. The locations of the draw-in points are illustrated in Fig. 31. All points of draw-in are located on the edge of the 
material blank. D1 and D3 are located at the center point of their respective sides. D2 is located at the corner of the material blank.

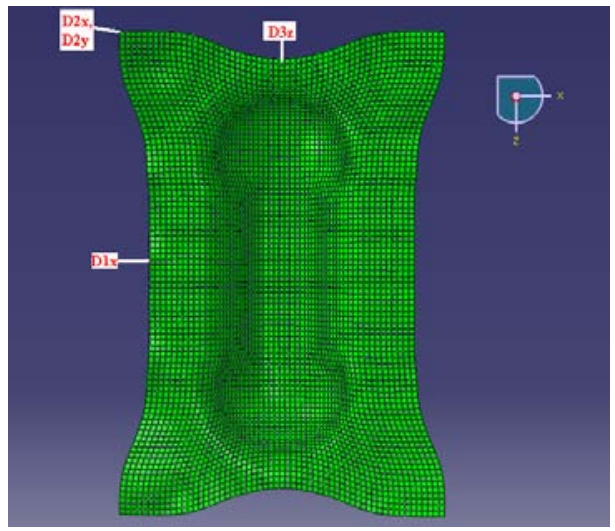

Fig. 31 Location of reported draw-in.

Shear angles are to be reported along the apex. The apex is defined as the line which passes through the points of greatest shear. Fig. 32 illustrates the location of the apex with a red line. The line begins at the vertical axis of symmetry and extends through the location of greatest shear angle all the way to the edge of the material blank.

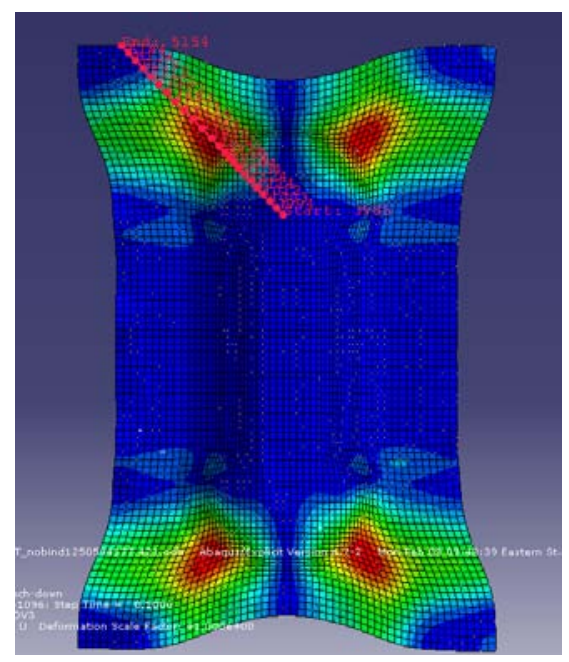

Fig. 32 Shear angle are to be measured along the apex line shown in red. 


\subsection{Participants}

Table 4 is a list of participants for the next round of simulations.

Table 4. Potential Participants

$\begin{array}{lll}\text { Name } & \text { Institution } & \text { Email } \\ \text { Jian Cao } & \text { Northwestern Univ, USA } & \text { jcao@northwestern.edu } \\ \text { James Sherwood } & \text { Univ of MA - Lowell, USA } & \underline{\text { James_Sherwood@uml.edu }} \\ \text { Philippe Boisse } & \text { INSA-Lyon, FRANCE } & \underline{\text { Philippe.Boisse@insa-lyon.fr }} \\ \text { Remko Akkerman } & \text { Univ of Twente, NETHERLANDS } & \underline{\text { r.akkerman@,ctw.utwente.nl }} \\ \text { Stepan Lomov } & \text { KU-Leuven, BELGIUM } & \underline{\underline{\text { Stepan.Lomov@mtm.kuleuven.be }}} \\ \text { Andy Long } & \text { Univ of Nottingham, UK } & \underline{\text { Andrew.Long@nottingham.ac.uk }} \\ \text { Tong-Xi Yu } & \text { HKUST, HONG KONG } & \underline{\text { metxyu@ ust.hk }} \\ \text { Daniel Leutz } & \text { Technical University of Munich } & \underline{\underline{\text { leutz@lcc.mw.tum.de }}} \\ \text { Phil Harrison } & \text { University of Glasgow } & \text { p.harrison@eng.gla.ac.uk }\end{array}$

\subsection{Benchmark Status}

The results of the Round 2 simulations were summarized in a paper [29] presented at the 2010 ESAForm conference held in April 2010 in Brescia, Italy. Based on the Round 2 results from the various research teams around the world, a set of parameters was developed for completing a Round 3. During the ESAForm 2010 conference, a meeting was held amongst the Benchmark participants attending the conference. Ideas for future work on modeling and friction were outlined. Sherwood and Chen volunteered to organize that information and send out a first draft for all to review.

The participants agreed to write a journal paper on the results of the Round 2 simulations. Sherwood and Chen will take the lead on collating the information for that paper using their ESAForm paper [29] on the benchmark results as a starting point. "Mini" descriptions will be submitted from each of the teams about their respective modeling approach for inclusion in the paper. The goal is to have the paper written and submitted by July 2010 .

\subsection{Friction Modeling}

Parametric studies of the thermostamping process using the Hersey number friction model and different binder forces have been completed to explore the effect of the in-plane tension on the wrinkling and tearing of the fabric. These studies demonstrated how the model could be used to determine the most appropriate binder force to avoid wrinkling (high shear strain within the fabric) and tearing (high tensile stress in the yarns).

The phenomenological friction models relating the dynamic coefficient of friction to the Hersey number were incorporated into ABAQUS/Explicit and LS-DYNA as user-defined friction subroutines in conjunction with userdefined material models. The user-defined friction subroutines were first validated using a finite element model of the experimental friction test. The dynamic coefficients of friction obtained from the finite element simulations correlated well with the experimentally obtained dynamic coefficients of friction. Static coefficients of friction were not included in the friction subroutines after a parametric study showed that the importance of the static friction coefficient contribution to the overall result is of minimal significance in comparison to the contribution of the dynamic coefficient of friction. The user-defined friction subroutines accounted for dynamically changing coefficients as a function of variations in velocities and normal forces experienced by the fabric.

It was found that capturing varying friction at the tool/fabric and fabric/fabric interfaces was important because the varying friction significantly affected the force required by the punch to form the part, as well as the resulting tensile stresses in the fabric yarns. A parametric study showed that increasing the stamping rate led to an increase in the friction force at the tool/fabric interface, thus increasing the punch force and the tensile stresses in the yarns. Using a constant coefficient of friction showed that the punch force had no dependence on the stamping rate. Incorrectly predicting the fabric stresses could lead to manufacturing defects such as fabric tearing and not optimizing the processing speed, i.e. manufacturing rate. 
After validating the user-supplied subroutines with a finite element model of the friction test, both VFRIC (ABAQUS) and USRFRC (LS-DYNA) were applied to a single-layer $\left(\left[0^{\circ} / 90^{\circ}\right]\right)$ hemisphere model with equal binder pressures and stamping rates, and the resulting punch forces were compared between ABAQUS/Explicit and LS-DYNA. Figure 33 shows that the results from the ABAQUS and LS-DYNA agree well with each other.

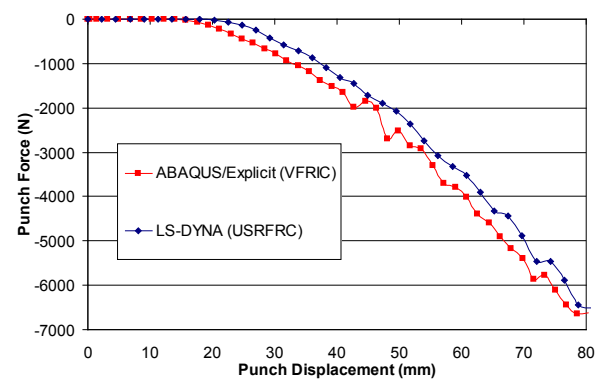

Fig. 33 Comparison of hemisphere model between ABAQUS and LS-DYNA for user-defined friction subroutines with a binder pressure of $200 \mathrm{kPa}$ and a stamping rate of $90 \mathrm{~mm} / \mathrm{s}$

It was found that as the friction increases the punch force required to deform the fabric will increase. From the experimental results, it was shown that as the velocities increase the frictional forces between the metal tooling and the fabric and between adjacent layers of fabric will increase. However, as the normal force between the fabric and the contacting surface increases, the effective friction coefficient decreases. Thus, it is expected that as the stamping rate is increased, then the punch forces required to deform the fabric may increase or decrease depending on the combination of speed and normal force. To explore the effect of stamping rate on the punch force, three different stamping rates were applied to the hemisphere model in ABAQUS/Explicit. Table 5 summarizes the stamping rates used, and Fig. 34 compares the punch forces at each stamping rate as a function of punch displacement. The differences in punch forces shown in Fig. 34 indicate that the punch force does increase with increasing punch velocity.

Table 5. Effect of punch velocity on punch force

$\begin{array}{ccc}\begin{array}{c}\text { Simulation } \\ \text { No. }\end{array} & \begin{array}{c}\text { Binder } \\ \text { Pressure } \\ (\mathrm{kPa})\end{array} & \begin{array}{c}\text { Punch } \\ \text { Velocity } \\ (\mathrm{mm} / \mathrm{s})\end{array} \\ 1 & 200 & 9.0 \\ 2 & 200 & 22.5 \\ 3 & 200 & 45.0\end{array}$



Fig. 34 Effect of punch velocity on punch force as a function of displacement using fabric-friction model 
Using the same stamping rates and binder pressures as given in Table 5 with a fixed coefficient of friction of 0.3 , the punch forces show no dependence on stamping rate. Thus, if the modeling is done using a constant coefficient of friction, the punch force can be either over-predicted or under-predicted relative to the results shown using a variable coefficient of friction, i.e. is a function of the velocity (Fig. 35). Fig. 35 shows the importance of incorporating a varying friction coefficient that is dependent upon the critical thermostamping process parameters such as stamping rate. As stated previously, higher frictional forces also increase the tensile stresses in the yarns in addition to the punch force. Fig. 36 shows that assuming a constant coefficient of friction $(\mu=0.3)$ greatly overpredicts the tensile stresses in the fabric yarns. Incorrectly predicting the fabric stresses could lead to manufacturing defects such as fabric tearing and not optimizing the processing speed, i.e. manufacturing rate.

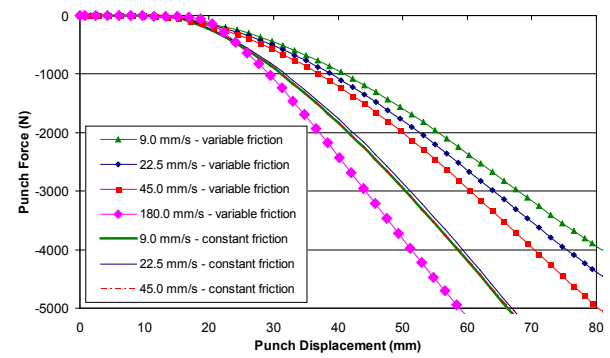

Fig. 35 Punch forces with the variable fabricfriction model and with constant friction coefficient $(\mu=0.3)$



(a)

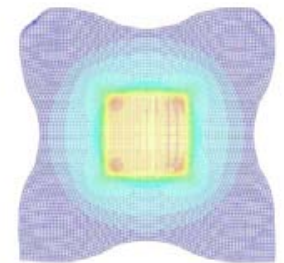

(b)

Fig. 36 Tensile stresses in fabric yarns with (a) fabric-friction model and with (b) constant friction coefficient $(\mu=0.3)$

\subsection{Modal Analysis}

Modal analysis was pursued as a means to quantify the correlation between the thermostamping simulation and actual parts made using the process. The modal analysis used the 3D Laser Vibrometer which was acquired with the funding made possible by the NSF Major Research Initiation Grant CMMI-0722469. The study was done for flat plates where the fabric had the yarns in their as-fabricated state, i.e. where warp and weft are mutually perpendicular, and in a sheared state, e.g. sheared $25^{\circ}$ from the as-fabricated weave orientation of $90^{\circ}$. For a threedimensional shape, the double-dome geometry as shown is Fig. 30 was used.

Some initial comparisons between the composite formed using the simulation and actual formed parts showed some promising results. The mode shapes and frequencies for the first and second modes are shown in Figs. 37 and 38, respectively. The frequencies calculated from the model are very close to the experimentally measured values. However, as can be seen in Fig. 39, there is a wide discrepancy between the model and the experimental frequencies for the third mode, which is the fourth mode in the model. The difference is most likely due to the bending stiffness 
being used for the beam elements in the hybrid mesh. Future work will be to explore this three-dimensional situation in more detail.

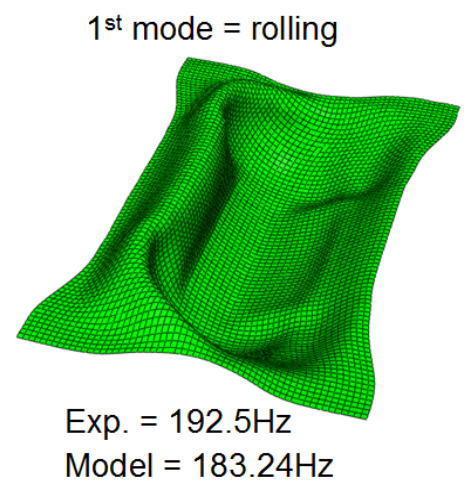

Fig. 37 Double Dome $1^{\text {st }}$ mode



Fig. 38 Double Dome $2^{\text {nd }}$ mode

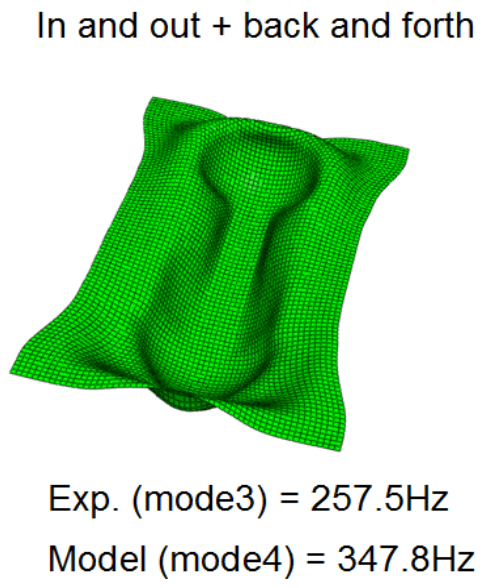

Fig. 39 Double Dome $3^{\text {rd }}$ mode

The use of modal analysis looked very promising based on the correlation between the model and the experimental data for the first and second modes, Due to the good agreement between the model and experimental data for the 
double-dome geometry (three-dimensional geometry) for some modes and not as good for another, it was concluded that the methodology should be used to investigate a less complex shape, i.e. a flat plate of the woven fabric (twodimensional geometry). The investigation of a flat plate would give the ability to develop a fundamental understanding of the parameters needed to achieve a complete description of the effective stiffness of a formed composite.

\subsection{Modal Analysis of a Flat Plate}

A flat plate was made with two layers of plain-weave fabric and analyzed experimentally using modal analysis and using the eigen-solution in ABAQUS [30]. The first three mode shapes for a flat plate correlated very well between model and experiment (Fig. 40). Note that due to the geometry of the square plate, the finite element model predicted repeated frequencies for modes 2 and 3, but the experiments showed an $8.1 \%$ difference between frequencies 2 and 3 (Table 6) for the actual plate.

The finite element model assumed a uniform thickness and uniform bending stiffness across the plate. Measurements of the formed plate showed that the plate was thicker on the perimeter in comparison to the center of the plate. This difference between center and edge thicknesses is reasonable because the force on the forming platen was applied in the center and thereby the edges of the top platen could "bend up". This slight bowing of the forming platen, while small, does explain how there could be a thickness gradient across the formed plate. Thus, the differences between the model and experimental frequencies are partially due to variations in thickness of the part as a result of fabrication and the model assuming a uniform thickness for the plate.

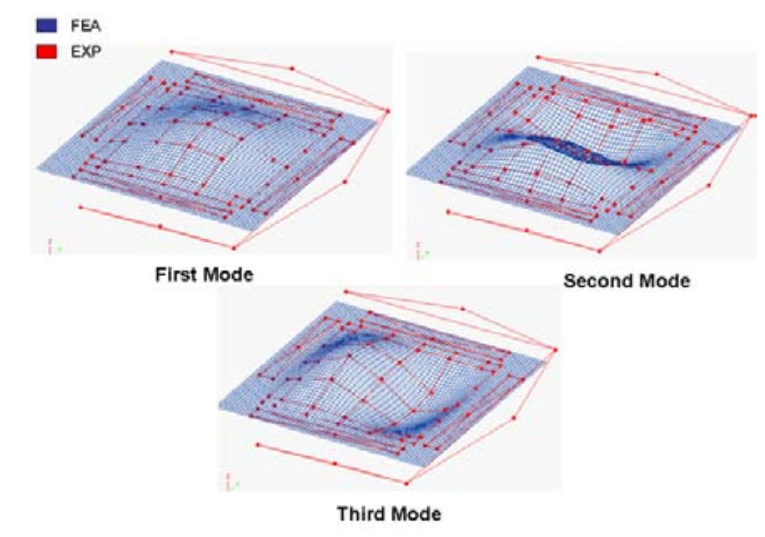

Fig. 40 Modal analysis of flat composite plate: Comparison of first three experimental and analytical mode shapes

Table 6. Comparison of first three frequencies between model and experiment

$\begin{array}{cccc}\text { Frequency } & \text { EXP }(\mathrm{Hz}) & \text { FEA }(\mathrm{Hz}) & \% \text { Diff. } \\ 1 & 117.44 & 110.00 & 6.34 \% \\ 2 & 213.99 & 226.26 & 5.73 \% \\ 3 & 232.88 & 226.26 & 2.84 \%\end{array}$

\subsection{Three-Point Bend Tests}

Warp and weft-orientation beam samples were cut from the middle and edge locations of the flat sheet and subjected to three-point bend tests. The measured flexural rigidities are summarized in Table 7. 
Table 7. Bending rigidity of composite plate cut samples

$\begin{array}{cccc}\text { Direction } & \begin{array}{c}\text { Edges - EI } \\ \left(\mathrm{N}-\mathrm{mm}^{2}\right)\end{array} & \begin{array}{c}\text { Middle - EI } \\ \left(\mathrm{N}-\mathrm{mm}^{2}\right)\end{array} & \text { \% Diff. } \\ \text { Warp } & 25,428 & 26,496 & 4.03 \% \\ \text { Weft } & 25,750 & 22,224 & 13.69 \% \\ \text { \% Diff. } & 1.25 \% & 16.12 \% & \end{array}$

At the edge of the plate, the bending rigidity varied by only $1.25 \%$ between warp and weft directions. However, a $16.12 \%$ difference was measured in the flexural rigidity between warp and weft directions in the middle of the plate. Also, the weft direction at the edge of the plate was $13.69 \%$ stiffer than the weft direction in the middle of the plate. The variation in the flexural stiffness at different locations in the plate and in different directions is probably the more significant source of the frequency differences between the finite element model and the experiment.

Future work will explore methods for including a more complete description of the warp and weft yarn behavior in the forming simulation and the potential pressure variation experienced by the part from the tool resulting in a thickness variation across the final part. Nevertheless, the model and experiment frequencies correlated within 10\%, so the proposed methodology of using modal analysis to compare the stiffness of the formed part to that found using the simulation does show promise and can aid in refining the geometry and material parameters used in the forming simulations.

\subsection{Fabric Bending}

As already discussed, the forming simulations show good correlation of the fabric deformation with the experimental data of the same geometry, e.g. hemisphere and automotive floor pan. However, this correlation may be improved by incorporating the bending stiffness of the fabric into the thermostamping process simulation. Thus, as a final task in this NSF-funded program, some initial research has been pursued to develop a fundamental understanding of fabric bending stiffness.

The bending research builds on the existing fabric bending methods by [31,32]. However, rather than concentrating on a numerical description of the bending stiffness, the current work focuses on extracting an empirical nonlinear moment-curvature relationship from digital images of fabric "beams" for implementation into the existing ABAQUS and LS-DYNA material models that have been developed by the UMass-Lowell research group [18, 20, 21, 28].

For the current research, 5-yarn-wide fabric beam samples were cut from the warp and weft directions of a woven fabric. The samples were marked to denote the $80,90,100,110$ and $120 \mathrm{~mm}$ locations as measured from the free end of the "beam". Next, the sample was positioned in a clamp, as shown in Figure 41, at the 80-mm mark, and an image of the bending fabric was captured with a digital camera. Similarly, the data have been collected for the other lengths to see how the effective length of the beam influences the resulting moment-curvature relation. The data are being analyzed for concluding the parameters for use in ABAQUS to include this mechanical behavior in the model.

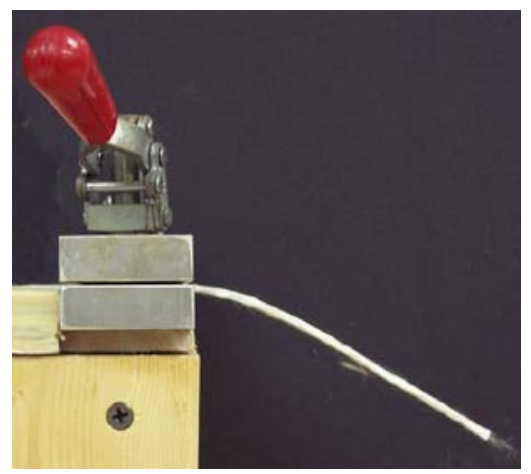

Figure 41: Fabric sample in the testing set-up. 


\subsection{Publications}

1. Gamache L., Sherwood J. A., Chen J., Cao J.: Characterization of the Fabric/Tool and Fabric/Fabric Friction during the Thermostamping Process. Proceedings of the 10th International ESAFORM Conference on Material Forming. Zaragoza, Spain, 2007.

2. Gamache L.: The Design and Implementation of a Friction Test Apparatus Based on the Thermostamping Process of Woven-Fabric Composites, MS Thesis, Department of Mechanical Engineering. Lowell, MA: University of Massachusetts Lowell, 2007.

3. Cao J., Akkerman R., Boisse P., Chen J., Cheng H. S., DeGraaf E. F., Gorczyca J., Harrison P., Hivet G., Launay J., Lee W., Liu L., Lomov S., Long A., Deluycker E., Morestin F., Padvoiskis J., Peng X. Q., Sherwood J., Stoilova T., Tao X. M., Verpoest I., Willems A., Wiggers J., Yu T. X., Zhu B.: Characterization of mechanical behavior of woven fabrics: experimental methods and benchmark results. Composites: Part A, 39:1037-1053, 2008.

4. Fetfatsidis K. A., Sherwood J. A., Chen J., Jauffres D.: Characterization of the fabric/tool and fabric/fabric friction during the thermostamping process. Proceedings of the 12th International Conference. Twente, Nederlands, 2009.

5. Jauffres D., Morris C. D., Sherwood J., Chen J.: Simulation of the thermostamping of woven composites: determination of the tensile and in-plane shearing behaviors. Proceedings of the 12th International ESAFORM Conference. Twente, Nederlands, 2009.

6. Jauffres D., Morris C. D., Kremer J., Sherwood J., Chen J. Simulation of the thermostamping of woven composites: mesoscopic modeling using FEA explicit codes. Proceedings of the 12th International ESAFORM Conference. Twente, Nederlands, 2009.

7. Jauffres D., Morris C. D., Sherwood J., Chen J.: Discrete mesoscopic modeling for woven fabric reinforcement forming simulation. International Journal of Material Forming: Vol. 2 Suppl 1:173-176, 2009.

8. Jauffres D., Sherwood J. A., Chen J., Morris C. D., and Kremer J.: Mesosocopic finite element modeling of woven reinforcements applied to sheet molding compound forming simulation. Proceedings of the 17th International Conference on Composite Materials. Edinburgh, UK, 2009.

9. Fetfatsidis, K.A.: Characterization of the Tool/Fabric and Fabric/Fabric Friction for Woven Fabrics: Static and Dynamic, MS Thesis, Department of Mechanical Engineering. Lowell, MA: University of Massachusetts Lowell, 2009.

10. Morris, C.D.: Simulation of the Thermostamping Process for Woven Fabric Composites using LS-DYNA, MS Thesis, Department of Mechanical Engineering. Lowell, MA: University of Massachusetts Lowell, 2010 (estimated).

11. Sargent J., Chen J., Sherwood J., Cao J., Boisse P., Willem A., Vanclooster K., Lomov S.V., Khan M., Mabrouki T., Fetfatsidis K., Jauffrès D., Benchmark Study of Finite Element Models for Simulating the Thermostamping of Woven-Fabric Reinforced Composites. Proceedings of the 13th International ESAFORM Conference. Brescia, Italy, 2010.

12. Fetfatsidis K.A., Gamache L., Sherwood J.A., Jauffrès D. and Chen J., Design of an Apparatus for Measuring Tool/Fabric and Fabric/Fabric Friction of Woven-Fabric Composites During the Thermostamping Process, International Journal of Material Forming, (submitted), 2010.

\subsection{Conclusions}

Yarn and fiber parameters were investigated and their ability to model the compaction behavior of commingled fibers has been demonstrated by their influence on the resulting shear load-deformation curve. A $3^{\text {rd }}$-generation friction-test apparatus using load-control to apply the normal force to the surface of the fabric has been designed, built and validated allowing for the static and dynamic coefficients of friction to be quantified for tool-fabric and fabric-fabric friction. A set of experiments has been developed to quantify the mechanical behavior of woven fabrics for the forming process. A set of constitutive models has been implemented in the ABAQUS and LS-DYNA explicit finite element codes. The ability of the numerical design tool to simulate the forming of composite parts for woven 
fabric composites has been demonstrated. The UMass-Lowell research group is participating in an international benchmark program with other research groups doing similar work in Europe and Asia. The design tool has been shown to have the ability to link the manufacturing process to the structural response of the formed part. This validation was done by comparing experimental and finite element results for structural stiffness and vibration response of simple and relatively complex parts. Studies have been initiated to explore the importance of the fabric bending stiffness in the forming of composite parts.

\subsection{Acknowledgements}

The authors would like to thank the NSF Division of Design, Manufacture, and Industrial Innovation (DMI\#0522923), U.S. Department of Energy, and Ford Motor Company (Dr. Patrick Blanchard) for their support of this research. The letter of support from General Motors for the grant proposal is also appreciated. The contributions of Dr. Lu Liu, UMass-Lowell graduate and post doc, MSME students: Lisa Gamache, Corey Morris, Konstantine Fetfatsidis, and James Kremer, and undergraduate students David Winchester, Joanna Langworthy and Joseph Sargent and Ezekial Radik are appreciated. The expertise of post doc Dr. David Jauffrès is especially appreciated in the completion of the overall project.

\subsection{References}

1. C.E. Wilks, "Characterization of the Tool/Ply Interface during Forming," PhD Thesis, School of Mechanical, Materials, Manufacturing and Management. Nottingham, UK: University of Nottingham, 1999.

2. A.E. Long, C.D. Rudd, M. Blagdon M., and P. Smith, "Characterizing the Processing and Performance of Aligned Reinforcements during Preform Manufacture,” Composites: Part A, pp. 27247-253, 1996.

3. L. Liu, J. Chen, J. Gorczyca, J. Sherwood, "Modeling of Friction and Shear in Thermostamping of Composites Part II," Journal of Composite Materials, vol. 38, pp. 1931-1947, 2004.

4. L. Liu, J. Chen, X. Li, and J. Sherwood, "Two-Dimensional Macro-Mechanics Shear Models of Woven Fabrics," Composites: Part A, vol. 36, pp.105-114, 2005.

5. L. Liu, J. Chen, J. Sherwood, "Analytical Model of Shear of 4-Harness Satin Weave Fabrics," American Institute of Physics Proceedings 712, 8th NUMIFORM Technical Conference, June 13-17, Columbus, OH, 2004.

6. A. Bulusu, "Modeling of Architecture and Deformation of Dry Woven Fabrics during Shear," MS Thesis, Department of Mechanical Engineering, Lowell, MA, University of Massachusetts Lowell, 2001.

7. I.M. Hutchings, Tribology: Friction and Wear of Engineering Materials, Ann Arbor, MI: CRC Press, 2002.

8. J. Gorczyca, "A Study of the Frictional Behavior of a Plain-Weave Fabric during the Thermostamping Process," DEng Dissertation, Department of Mechanical Engineering, Lowell, MA, University of Massachusetts Lowell, 2004.

9. J. Gorczyca-Cole, J.A. Sherwood and J. Chen, "A Friction Model for Thermostamping Commingled GlassPolypropylene Woven Fabrics,” Composites: Part A, vol. 38, pp. 393-406, 2007.

10. J. Gorczyca, J. Sherwood, L. Liu, and J. Chen, "Modeling of Friction and Shear in Thermostamping Process Part I," Journal of Composite Materials, vol. 38, pp. 1911-1929, 2004.

11. S. Chow S., "Frictional Interaction between Blank Holder and Fabric in Stamping of Woven Thermoplastic Composites.," MS Thesis, Department of Mechanical Engineering, Lowell, MA: University of Massachusetts Lowell, 2002.

12. L. Gamache, "The Design and Implementation of a Friction Test Apparatus Based on the Thermostamping Process of Woven-Fabric Composites," MS Thesis, Department of Mechanical Engineering, Lowell, MA, University of Massachusetts Lowell, 2007.

13. L. Gamache, J.A. Sherwood, J. Chen, J. Cao, "Characterization of the Fabric/Tool and Fabric/Fabric Friction during the Thermostamping Process," Proceedings of the 10th International ESAFORM Conference on Material Forming, Zaragoza, Spain, 2007. 
14. Fetfatsidis K. A., Sherwood J. A., Chen J., Jauffres D.: Characterization of the fabric/tool and fabric/fabric friction during the thermostamping process. Proceedings of the 12th International Conference on Material Forming. Twente, Nederlands, 2009.

15. Fetfatsidis, K.A.: Characterization of the Tool/Fabric and Fabric/Fabric Friction for Woven Fabrics: Static and Dynamic, MS Thesis, Department of Mechanical Engineering. Lowell, MA: University of Massachusetts Lowell, 2009.

16. Morris, C.D.: Simulation of the Thermostamping Process for Woven Fabric Composites using LS-DYNA, MS Thesis, Department of Mechanical Engineering. Lowell, MA: University of Massachusetts Lowell, 2009.

17. Willems A., Lomov S., Vandepitte D., Verpoest I.: Double dome forming of woven textile composites. Proceedings of the 9th International ESAFORM Conference on Material Forming. Glasgow, UK, 2006.

18. Jauffres D., Morris C. D., Sherwood J., Chen J.: Simulation of the thermostamping of woven composites: determination of the tensile and in-plane shearing behaviors. Proceedings of the 12th International ESAFORM Conference on Material Forming. Twente, Nederlands, 2009.

19. Cao J., Akkerman R., Boisse P., Chen J., Cheng H. S., DeGraaf E. F., Gorczyca J., Harrison P., Hivet G., Launay J., Lee W., Liu L., Lomov S., Long A., Deluycker E., Morestin F., Padvoiskis J., Peng X. Q., Sherwood J., Stoilova T., Tao X. M., Verpoest I., Willems A., Wiggers J., Yu T. X., Zhu B.: Characterization of mechanical behavior of woven fabrics: experimental methods and benchmark results. Composites: Part A, 39:1037-1053, 2008.

20. Jauffres D., Morris C. D., Kremer J., Sherwood J., Chen J. Simulation of the thermostamping of woven composites: mesoscopic modeling using FEA explicit codes. Proceedings of the 12th International ESAFORM Conference on Material Forming. Twente, Nederlands, 2009.

21. Jauffres D., Morris C. D., Sherwood J., Chen J.: Discrete mesoscopic modeling for woven fabric reinforcement forming simulation. International Journal of Material Forming: (in publication), 2009.

22. Boisse P., Hamila N., Helenon F., Hagege B., Cao J.: Different approaches for woven composite reinforcement forming simulation. International Journal of Material Forming, 1:21-29, 2008.

23. Li X.: Material characterization of woven-fabric composites and finite element analysis of the thermostamping process. DEng Dissertation, Department of Mechanical Engineering. Lowell, MA: University of Massachusetts Lowell, 2005.

24. Bathe K. J.: Finite Element Procedures. Englewood Cliffs: New Jersey: Prentice Hall, 1996.

25. Peng X. Q., Cao J.: A continuum mechanics-based non-orthogonal constitutive model for woven composite fabrics. Composites: Part A, 36:859-874, 2005.

26. Lomov S., Boisse P., Deluycker E., Morestin F., Vanclooster K., Vandepitte D., Verpoest I., Willems A.: Fullfield strain measurements in textile deformability studies. Composites: Part A, 39:1232-1244, 2008.

27. Harrison P., Clifford M. J., Long A.: Shear characterization of viscous woven textile composites: a comparison between picture frame and bias extension experiments. Composites Science and Technology, 64:1453-1465, 2004.

28. Jauffres D., Sherwood J. A., Chen J., Morris C. D., and Kremer J.: Mesosocopic finite element modeling of woven reinforcements applied to sheet molding compound forming simulation. Proceedings of the 17th International Conference on Composite Materials. Edinburgh, UK, 2009.

29. Sargent J., Chen J., Sherwood J., Cao J., Boisse P., Willem A., Vanclooster K., Lomov S.V., Khan M., Mabrouki T., Fetfatsidis K., Jauffrès D., Benchmark Study of Finite Element Models for Simulating the Thermostamping of Woven-Fabric Reinforced Composites. Proceedings of the 13th International ESAFORM Conference. Brescia, Italy, 2010.

30. Sherwood J., Fetfatsidis K., Winchester D., Jauffrès D., Avitabile P., Chen J., Using Modal Analysis to Investigate the Validity of Finite Element Models for Simulating the Thermostamping of Woven-Fabric Reinforced Composites. Proceedings of the 13th International ESAFORM Conference. Brescia, Italy, 2010. 
31. T.G. Clapp, H. Peng, T.K. Ghosh and J.W.Eischen. Indirect Measurement of the Moment-Curvature Relationship for Fabrics. Textile Research Institute, 525-533, September 1990.

32. E.de Bilbao, D. Soulat, G. Hivet and A. Gasser. Experimental Study of Bending Behaviour of Reinforcements. Experimental Mechanics, March 2009. 
Annual Report for Period:09/2007 - 08/2008

Principal Investigator: Papathanasiou, Thanasis D.

Organization: USC Research Foundation

Title:

A Hierarchical, Structure-Oriented and Stochastic Approch to Model Liquid Molding Processes
Submitted on: 06/07/2008

Award ID: 0522221

\section{Senior Personnel}

\section{Project Participants}

Name: Papathanasiou, Thanasis

Worked for more than 160 Hours: Yes

Contribution to Project:

Name: Paleologos, Evan

Worked for more than 160 Hours: $\quad$ No

\section{Contribution to Project:}

Dr. Paleologos has notified me that he has accepted a faculty position at an overseas University and that has to terminate his participation in this grant. Following approval of the NSF program director, an ammended statement of work and rebudget has been proposed. Specifically, $80 \%$ of the funds budgeted for Dr. Paleologos in 2007-2008 will be used for post doc salary and $20 \%$ for materials and supplies.

\section{Post-doc}

\section{Name: Chen, Xiaoming}

Worked for more than 160 Hours: Yes

Contribution to Project:

Dr. Chen is funded by NSF grant DMI-0522221. He work developing code and running simulations pertaining to this award.

\section{Graduate Student}

\section{Undergraduate Student}

Name: McKenzie, Daniel

Worked for more than 160 Hours: $\quad$ Yes

Contribution to Project:

Dan was involved in the IREE supplement to this grant and has contributed in the design and fabrication of the flow cells which were used in the context of that award. Along with the PI, he visited Lulea University of Technology (Sweden) to carry out flow visualization experiments.

\section{Technician, Programmer}

\section{Other Participant}

Research Experience for Undergraduates

Organizational Partners

\section{Other Collaborators or Contacts}




\section{Activities and Findings}

\section{Research and Education Activities: (See PDF version submitted by PI at the end of the report)}

Our research activities in the previous year were focused on the following:

(1)Developed, tested and validated computer codes for the generation of model geometries having user-specified microstructural characteristics. These are used as inputs to our fluids solver and consist of assemblies of fiber cross-sections. In the same context, we have continued our work to propose and test metrics which quantify microstructural features such as fiber clustering.

(2)Continued to investigate the effects of microstructure on the permeability of unidirectional fibrous media, using the previously developed 2D parallel boundary element codes. Specifically, the effects of non-uniform inter-tow and intra-tow fiber packing and random fiber clustering were studied.

(3)Started to implement the Fast Multipole Boundary Element Method. The FMBEM for solving two-dimensional potential flow problems has been applied to study transport in composite membranes which contain variously oriented flakes.

In the same period we presented results of our work at two conferences, namely the SPE's ANTEC meeting in Cincinnati, 5/2007 and the SAMPE-2007 meeting in Baltimore, 6/2007.

\section{Findings: (See PDF version submitted by PI at the end of the report)}

Our results in this report period were in the following topics:

(1)Computation of the effects of microstructure on the permeability of unidirectional fibrous media. Specifically, we studied the influence of non-uniform inter-tow and intra-tow fiber packing, as well as the influence of (unstructured) fiber clustering on permeability.

(2)Quantification of fiber clustering using a metric derived from Ripley's K-function.

(3)Use a Fast Multipole Boundary Element Method code to investigate the effect of internal structure

on the mass flux across random arrays of impermeable flakes.

During the report period the following papers were published:

X. Chen and T.D. Papathanasiou, 'The transverse permeability of disordered fiber arrays: A statistical correlation in terms of the mean nearest interfiber spacing', Transport in Porous Media, appeared in 71, 233-251,2008.

X. Chen and T.D. Papathanasiou, 'Barrier properties of flake-filled membranes: Review and numerical evaluation', J. of Plastic Film and Sheeting, 23(4), 319-346, 2007

Chen X. and T.D. Papathanasiou, 'Micro-Scale Modeling of Axial Flow through Unidirectional Disordered Fiber Arrays', Composites Science and Technology, 67 (2007) 1286-1293

Chen X, Mckenzie D. W., Papathanasiou T. D., 'Fiber clustering: its quantification and influence on unidirectional fibrous media', Proceedings to SAMPE-2007, Baltimore, MD, June 2007

Chen X, Papathanasiou T.D. 'Permeability of flake-filled membranes: a numerical evaluation', Proceedings to ANTEC-2007, Cincinnati, OH, May 2007, pp. 2349-2354

\section{Training and Development:}

Both the PI and the Post Doc have gained significant additional experience on the parallel implementation of the BEM method as a result of this award.

\section{Outreach Activities:}

\section{Journal Publications}

Xiaoming Chen and T.D. Papathanasiou, "The transverse permeability of disordered fiber arrays: A statistical correlation in terms of the mean interfiber spacing", Transport in Porous Media, accepted 3/2007, p. , vol. , ( ). Accepted, 
X. Chen and T.D. Papathanasiou, "Micro-Scale Modelling of Axial Flow through Unidirectional Disordered Fiber Arrays", Composites Science and Technology, p. 1286-1293, vol. 67, (2007). Published,

X.Chen and T.D.Papathanasiou, "Permeability of Flake-filled Barrier Membranes: A Numerical Evaluation", SPE ANTEC-2007 Proceedings, paper \# 0621, p. 621, vol. , (2007). Published,

\section{Books or Other One-time Publications}

\section{Web/Internet Site}

\section{Other Specific Products}

\section{Contributions}

\section{Contributions within Discipline:}

\section{Contributions to Other Disciplines:}

\section{Contributions to Human Resource Development:}

Continued training of Dr. Chen in parallel computing using the Boundary Element Method

Training of two undegraduate students (Dan MacKenzie and Fun Wai) in the numerical techniques and physical issues involved in this project. Both students enrolled in this research in teh context of a ECHE499 'individual study' project under the PI's supervision.

\section{Contributions to Resources for Research and Education:}

Contributions Beyond Science and Engineering:

\section{$\underline{\text { Special Requirements }}$}

Special reporting requirements: None

Change in Objectives or Scope: None

Animal, Human Subjects, Biohazards: None

\section{Categories for which nothing is reported:}

Organizational Partners

Activities and Findings: Any Outreach Activities

Any Book

Any Web/Internet Site

Any Product

Contributions: To Any within Discipline

Contributions: To Any Other Disciplines

Contributions: To Any Resources for Research and Education

Contributions: To Any Beyond Science and Engineering 
Results Report submitted

for project:

"A hierarchical, structure-oriented and stochastic approach to model liquid molding processes"

Award Number:

DMI-0522221

PI:

Thanasis D. Papathanasiou

Department of Chemical Engineering

University of South Carolina

Columbia, SC 29208

\section{Summary}

In the following we outline our results in the following areas:

(1) Computation of the effects of microstructure on the permeability of unidirectional fibrous media. Specifically, the effects of non-uniform inter-tow and intra-tow fiber packing and random fiber clustering were studied.

(2) Quantification of fiber clustering using a metric derived from Ripley's K-function.

(3) Benchmarking of the performance of a parallel boundary element code for solving three-dimensional Stokes flow problems using two different matrix assembly parallelization strategies.

(4) Use a Fast Multipole Boundary Element Method code to investigate the effect of internal structure on the mass flux across random arrays of impermeable flakes.

In the same period the following papers were published or accepted:

Chen X. and T.D. Papathanasiou, "The transverse permeability of disordered fiber arrays: A statistical correlation in terms of the mean interfiber spacing", Transport in Porous Media, accepted, 3/2007

Chen X. and T.D. Papathanasiou, "Micro-Scale Modelling of Axial Flow through Unidirectional Disordered Fiber Arrays", Composites Science and Technology, 67 (2007) 1286-1293 
In the same period the following paper appeared in the 2007 ANTEC Conference proceedings

Chen X, Papathanasiou T.D., Permeability of Flake-filled Barrier Membranes: A Numerical Evaluation, ANTEC Paper \#0621, Cincinnati, OH, USA, May 7-11 2007.

\section{Transverse Permeability of Perturbed Dual-scale Structures}

\subsection{Non-uniform Intra-tow Fiber Packing}

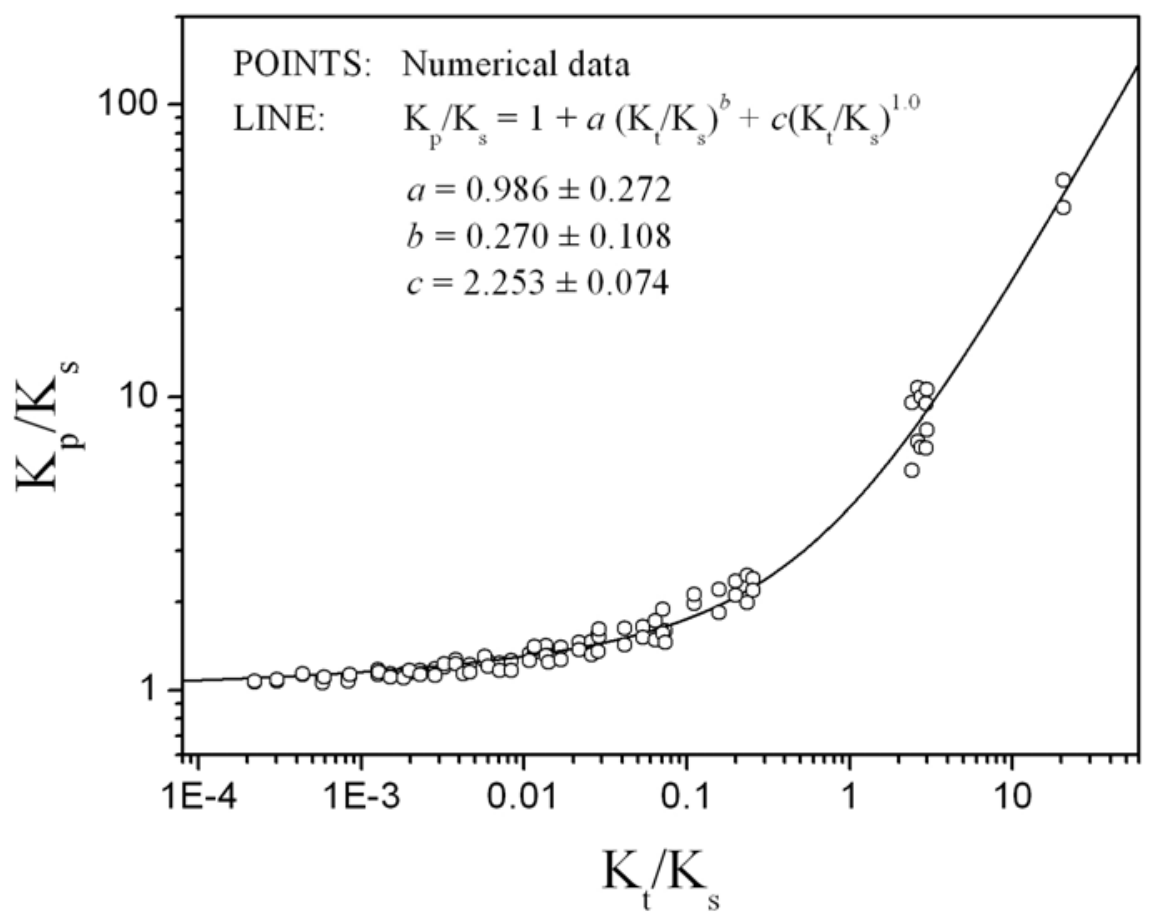

Fig.1. Master curve for the transverse permeability of a square array of circular fiber tows with nonuniform intra-tow fiber packing.

Numerical computations were carried out for the transverse permeability of a square array of circular fiber tows with non-uniform intra-tow fiber packing. The system of a square array of fiber tows is characterized by the global porosity $\phi$, the tow porosity $\phi_{\mathrm{t}}$ and the inter-tow porosity $\phi_{\mathrm{s}}$. Corresponding are the global permeability $K_{\mathrm{p}}$, tow permeability $K_{\mathrm{t}}$ and the permeability $K_{\mathrm{s}}$ of the same system in which the tows are strictly 
impermeable. Two limiting cases were studied at each fixed $\phi_{\mathrm{t}}$, the least uniform intratow fiber packing corresponding to $\delta_{\min }=0.05 D_{\mathrm{f}}$ and the most uniform packing corresponding to the maximum $\delta_{\min }$ achieved at each porosity level. A total of 94 geometries were generated; the corresponding $K_{\mathrm{p}}$ data were normalized with $K_{\mathrm{s}}$ and then plotted against $K_{\mathrm{t}} / K_{\mathrm{s}}$, as shown in Fig.1. Since $K_{\mathrm{t}}$ is generally unknown, in Fig. 1 it was replaced by $K_{\text {hex }}$, the permeability of the corresponding hexagonal array. Both $K_{\mathrm{t}}$ and $K_{\mathrm{s}}$ are therefore functions of porosity only. Apparently, the relation between $K_{\mathrm{p}} / K_{\mathrm{s}}$ and $K_{\mathrm{t}} / K_{\mathrm{s}}$ is nonlinear. In general, $K_{\mathrm{p}} / K_{\mathrm{s}}$ may be expressed as a series expansion of $K_{\mathrm{t}} / K_{\mathrm{s}}$ in the form

$$
\frac{K_{p}}{K_{s}}=a_{0}+\sum_{i=1}^{n} a_{i}\left(\frac{K_{t}}{K_{s}}\right)^{b_{i}}
$$

where the coefficients $a_{i}$ and the power indices $b_{i}$ can be determined by asymptotic considerations and data fitting. It suffices to use the simplest possible form of Eq.(1) to regress the data points in Fig.1, which is

$$
\frac{K_{p}}{K_{s}}=1+a\left(\frac{K_{t}}{K_{s}}\right)^{b}+c\left(\frac{K_{t}}{K_{s}}\right)
$$

where the correctness of asymptotic limits are guaranteed by the first term and the third term, and the coefficient $c$ expresses the effect of microstructure. In Fig.1, the fit of data to Eq. (2) is reasonably good. It can also be observed that

It can also be inferred from Fig. 1 that the permeability values corresponding to different intra-tow packing geometries at the same combinations of $\phi_{\mathrm{t}}$ and $\phi_{\mathrm{s}}$ are different. In Fig.2, the ratio $\xi$ between $K_{\mathrm{p} \text {,uniform }}$ and $K_{\mathrm{p} \text {,nonuniform }}$ is plotted as a function of $K_{\mathrm{t}} / K_{\mathrm{s}}$, where $K_{\mathrm{p} \text {,uniform }}$ is the permeability resulted from a uniform intra-tow fiber packing and $K_{\mathrm{p}, \text { nonuniform }}$ is the permeability resulted from a non-uniform intra-tow fiber packing. It is found that in most cases $\xi<1$, except at $\phi_{\mathrm{t}}=0.30$ (corresponding to a very densely packed geometry). 


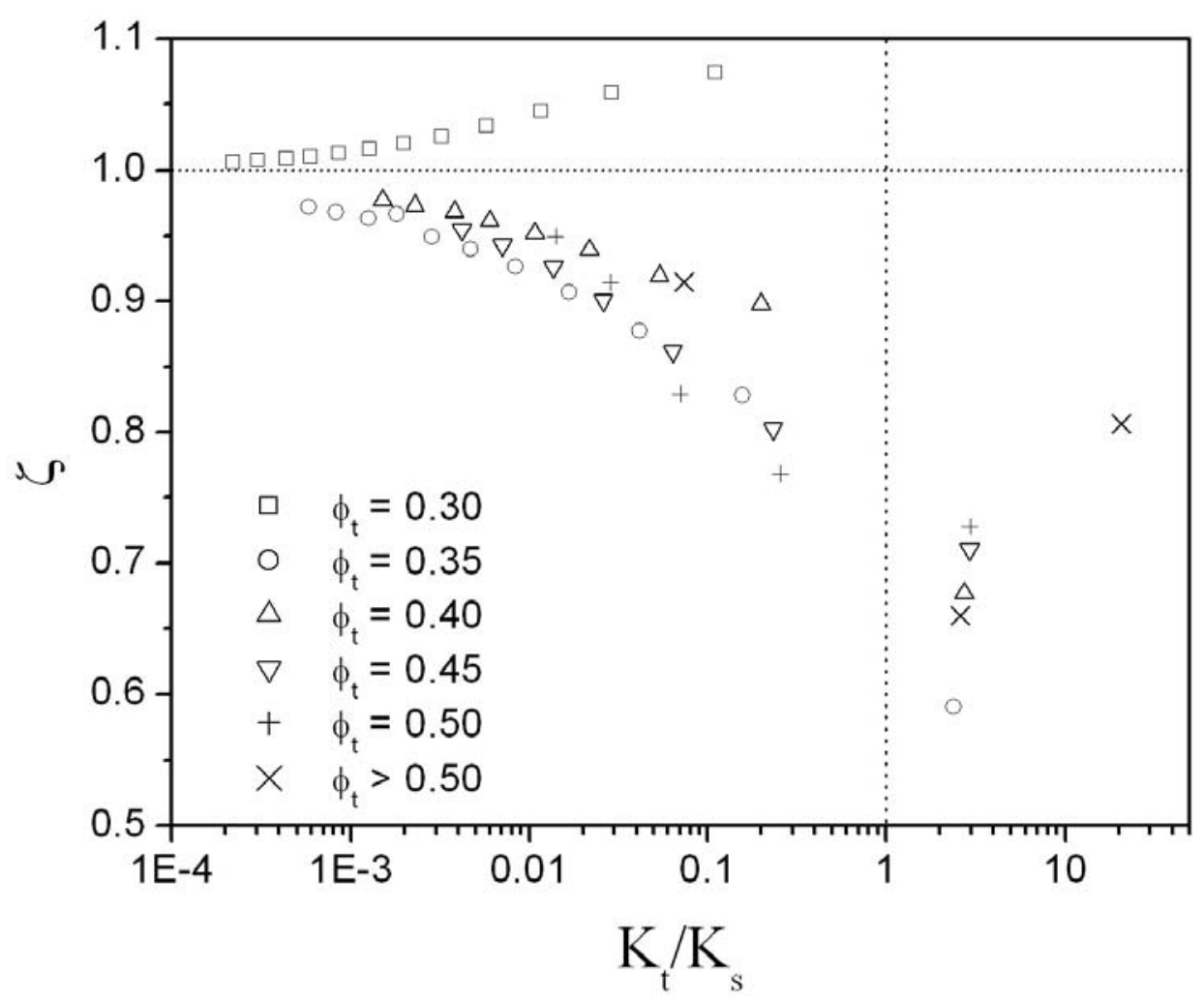

Fig.2 Effect of non-uniform intra-tow packing on the scatter of permeability data

\subsection{Non-uniform Fiber Bundle Spacing}

Fig. 3(a) and Fig. 4(a) show the calculated average permeabilities, as well as the associated standard deviations, as a function of $\Delta_{\min }$ and $\Lambda_{\max }$, respectively. These correspond to the microstructures described in section 2.1 in the activity report. Simulations on more than 200 geometries indicate that permeability increases with the extent of perturbation. This is better understood by comparing Fig. 3(b) to Fig. 3(c), and Fig. 4(b) to Fig. 4(c), where the speed contours in unperturbed systems as well as in perturbed systems are shown. It is clear by these speed contours that perturbations result in enlarged flow paths. Fig. 3(a) and Fig. 4(a) also show that the permeability assumes a broader distribution with increased perturbation extent. This effect is more pronounced 
when the vertical gap between rows of fiber tows is perturbed; the average permeability may change as large as $100 \%$ as compared to that of an unperturbed system.

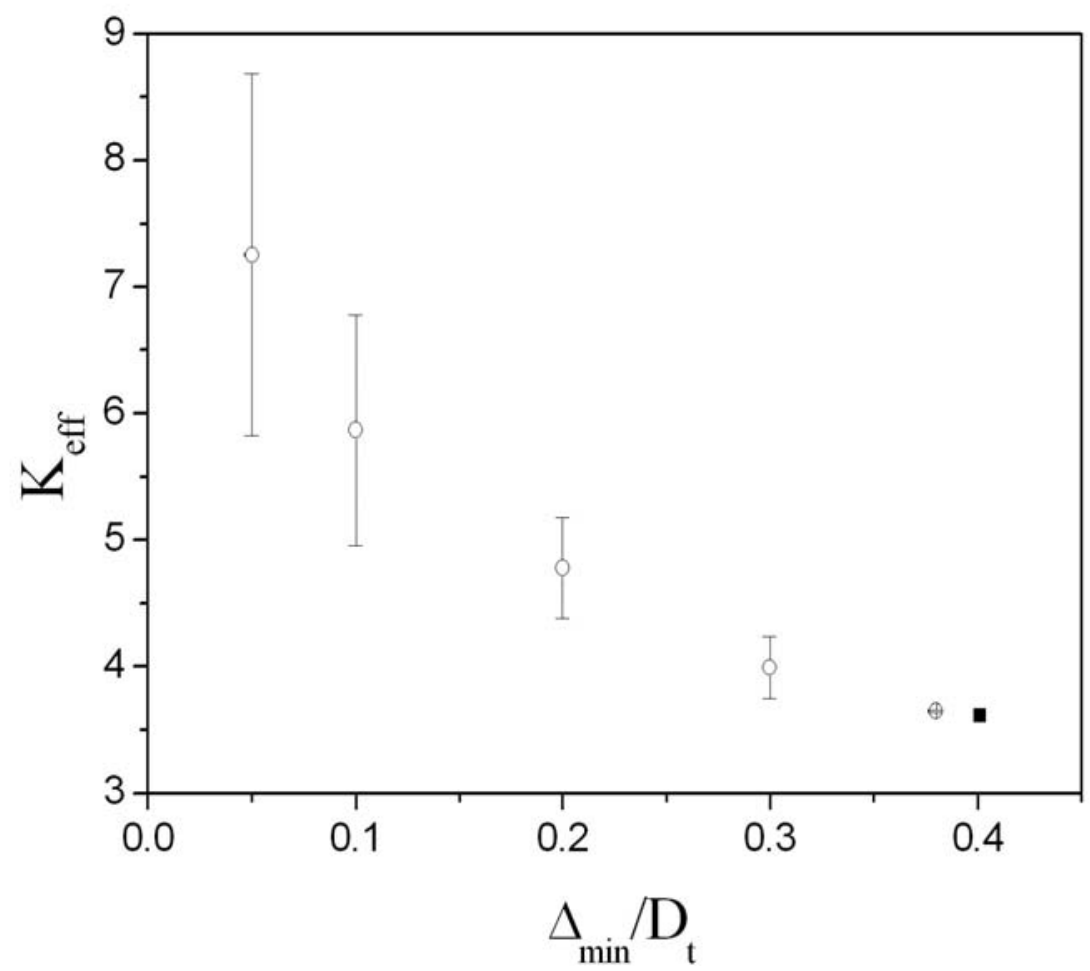

(a)

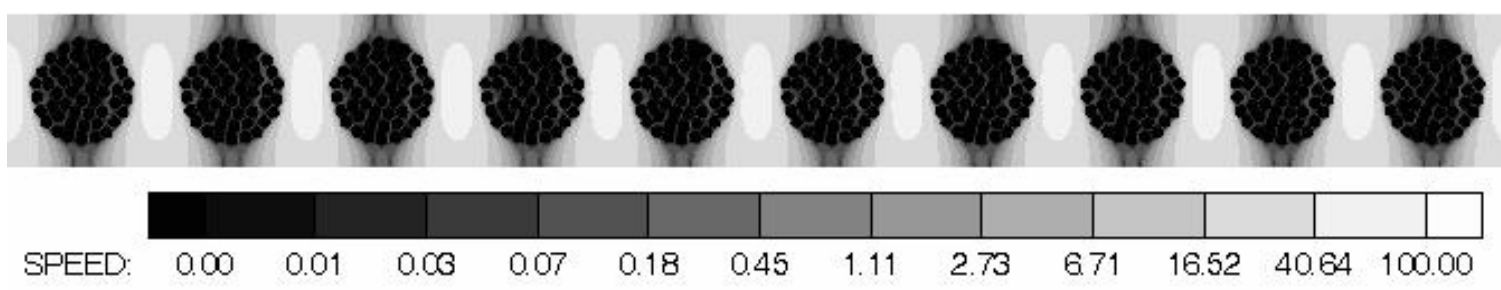

(b)
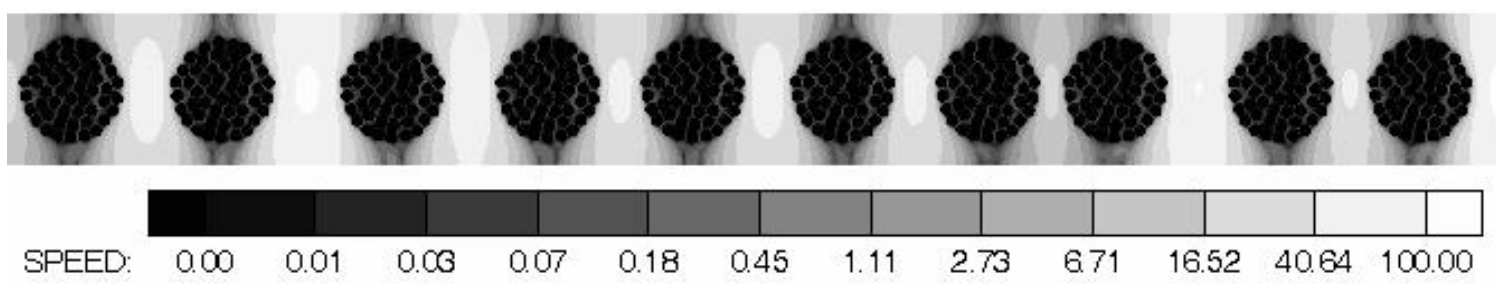

(c)

Fig.3. (a) Permeability of perturbed fiber bundles as a function of the horizontal perturbation extent, $\Delta_{\min } / \mathrm{D}_{t}$; (b) Speed contour of vertical flow through uniformly spaced fiber tows; (c) Speed contour of vertical flow through horizontally perturbed fiber tows. 


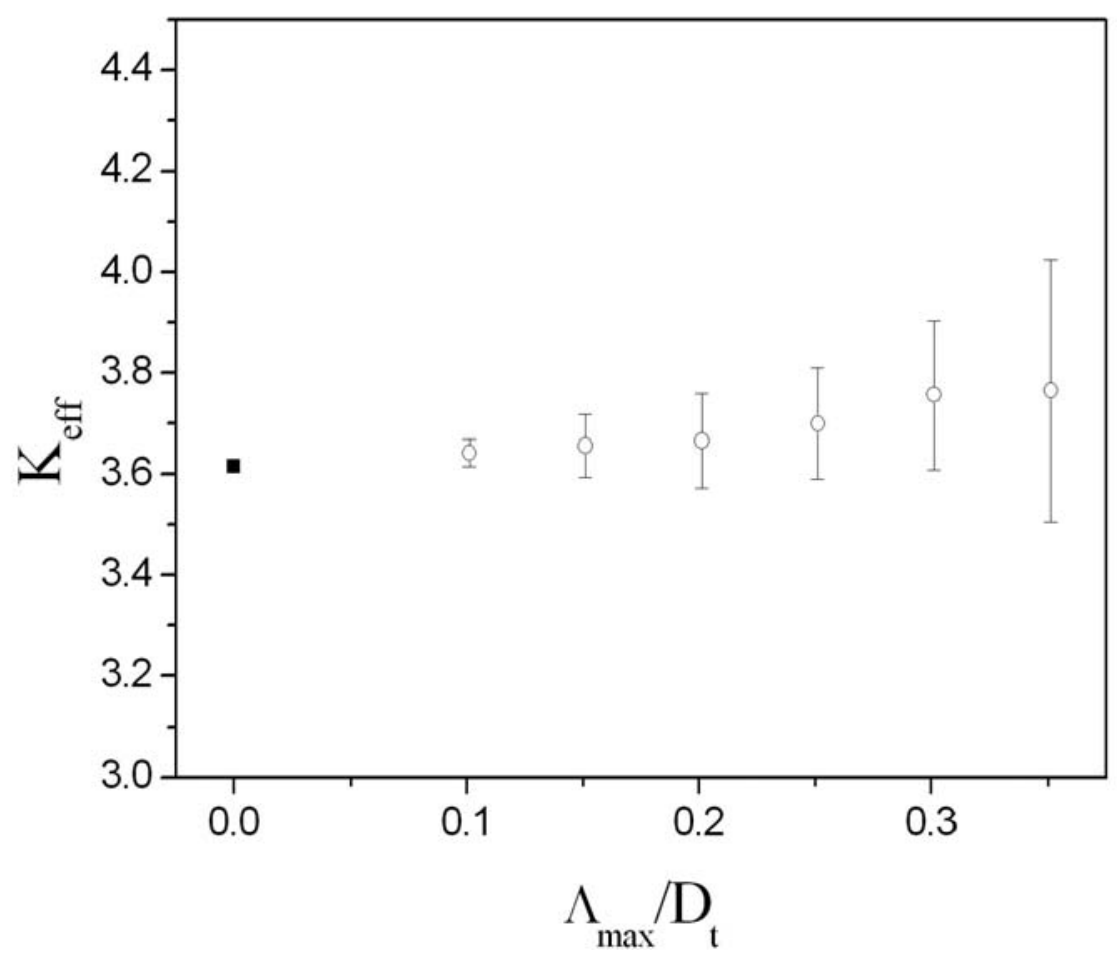

(a)

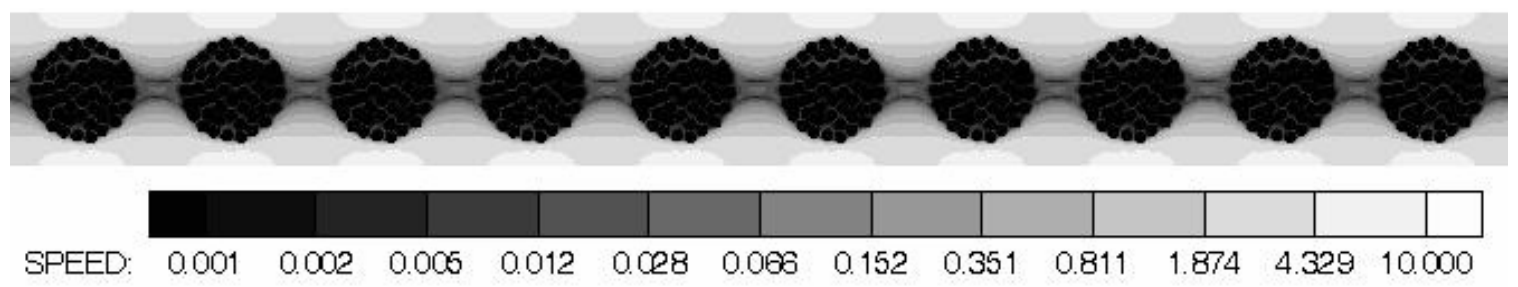

(b)



(c)

Fig.4. (a) Permeability of perturbed fiber bundles as a function of the vertical perturbation extent, $\Lambda_{\max } / D_{t}$; (b) Speed contour of horizontal flow through uniformly spaced fiber tows; (c) Speed contour of horizontal flow through vertically perturbed fiber tows. 


\section{Microstructure Characterization}

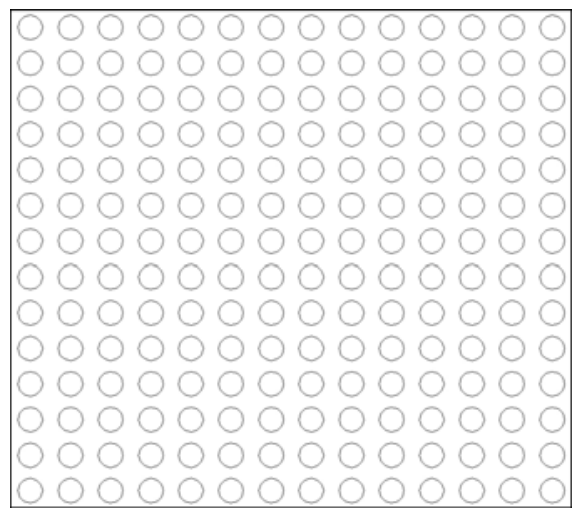

(a)



(c)

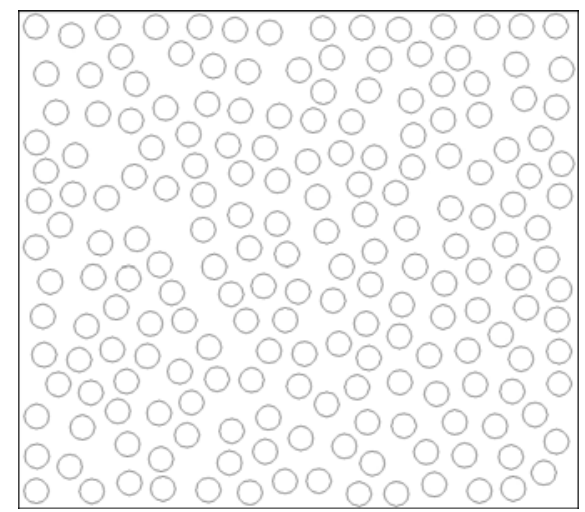

(b)

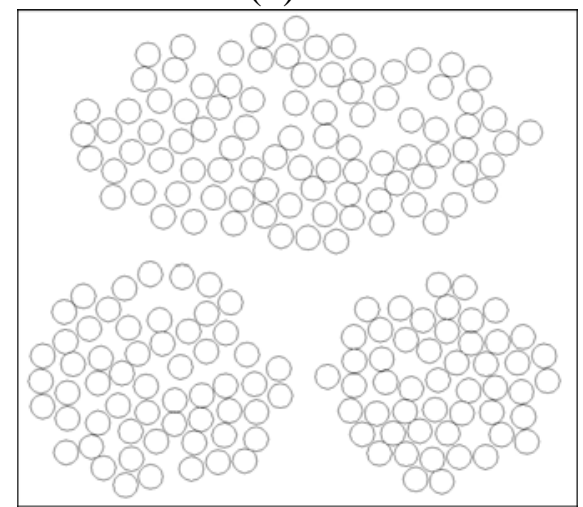

$(d)$

Fig.5. Four example fiber distributions. (a) regular fiber array, (b) hard-core fiber array, $\delta_{\min }=0.3 D_{\mathrm{f}}$; (c) hard-core fiber array, $\delta_{\min }=0.05 D_{\mathrm{f}}$; (d) three distinct fiber clusters.

Table 1: The measure of deviation $(\chi)$ calculated for the four distributions.

\begin{tabular}{lllll}
\hline Parameter/Model & Square array & Hard-core (I) & Hard-core (II) & Clustered \\
\hline Measure of deviation & 0.654 & 0.711 & 0.841 & 1.113 \\
\hline
\end{tabular}

The measure of deviation $\chi$ was first applied to quantify four example fiber distributions as shown in Fig.5. The results are given in Table 1. Based on visual observation, it can be established that the extent of fiber clustering increases from Fig. 5(a) to Fig. 5(d). This order is in agreement with what can be concluded based on the $\chi$ values of the four distributions. 


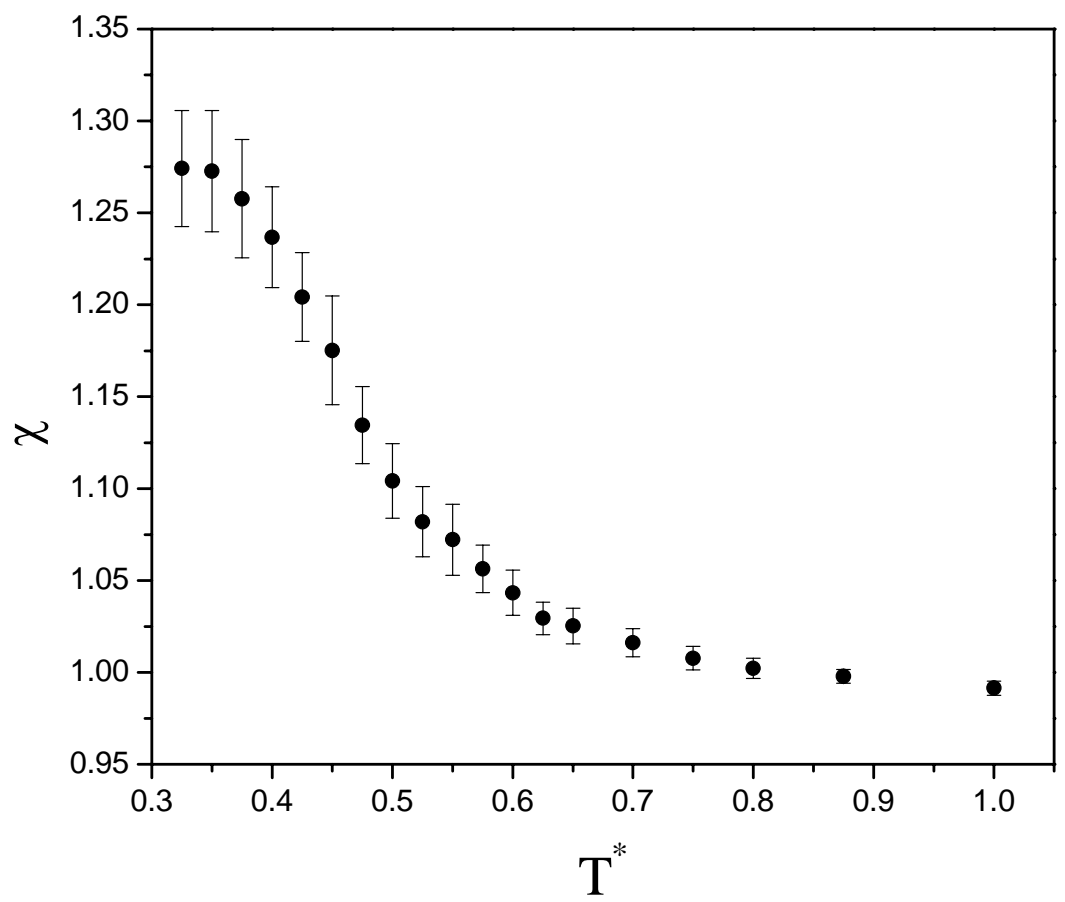

Fig.6. Correlation between $\chi$ and $\mathrm{T}^{*}$ for the microstructure generated by the NVT Monte Carlo Method

The measure of deviation $\chi$ was also applied to quantify the microstructure generated by the NVT Monte Carlo method. The correlation between $\chi$ and $T^{*}$ is shown in Fig. 6, where the data points shown are values averaged over 50 realizations of microstructures produced at the same $T^{*}$. In the range $0.3<T^{*} \leq 1.0$, it is shown that $\chi$ decreases monotonically as $T^{*}$ increases. Outside this range, $\chi$ does not seem to vary much with $T^{*}$ as the curve becomes flattened. The physical behavior of the Monte Carlo generated system is anticipated to show fiber aggregations at low $T^{*}$ and randomness at high $T^{*}$. The $\chi$ correctly indicates this physical behavior.

\section{Parallel Implementation of a 3D BEM Code}

Two codes, one based on the 'Owner-Compute' algorithm and the other based on the 'Distribute and Transfer' algorithm have been implemented. These codes have been tested on a Beowulf cluster (daniel.cse.sc.edu) that is equipped with 32 single $933 \mathrm{MHz}$ Intel Pentium III CPUs and a standard Ethernet interconnect. 
Table 2 summarizes the timing results for the execution of the two algorithms for a test problem of 2651 unknowns. It is noted that the matrix generation times are very different between the two. In terms of matrix assembly time, the 'Owner-Compute' algorithm is far more inferior to the other algorithm. Obviously, the solution times do not differ much as the same solver was used. It is also noted that the time spent on the matrix assembly exceeds the time spent on the solver in both cases, indicating the matrix assembly phase is the most expensive part in the 3D BEM code.

Table 2. Time results for the execution of the two parallel algorithms for a test problem of 2651 d.o.f.'s. The processor grid used is square.

\begin{tabular}{llll}
\multicolumn{4}{c}{ (a) Algorithm I, 'Owner-Computes' } \\
\hline CPU & matrix assembly & solver & total \\
\hline 1 & 15529.73 & 121.32 & 15651.24 \\
4 & 3957.62 & 38.13 & 3995.85 \\
9 & 1855.30 & 17.30 & 1872.73 \\
16 & 997.65 & 11.86 & 1009.63 \\
25 & 641.12 & 7.42 & 648.69 \\
\hline \multicolumn{4}{c}{ (b) Algorithm II, 'Distribute and Transfer’ } \\
\hline CPU & matrix assembly & solver & total \\
\hline 1 & 854.81 & 121.35 & 976.58 \\
4 & 422.27 & 38.24 & 460.85 \\
9 & 247.50 & 17.26 & 265.14 \\
16 & 157.59 & 11.91 & 169.89 \\
25 & 119.13 & 8.60 & 128.10 \\
\hline
\end{tabular}

\section{Application of the 2D Fast Multipole Boundary Element Method}

Direct numerical simulations are conducted in two dimensions to compute the barrier improvement factor of membranes filled with aligned flakes. The effects of flake aspect ratio, volume fraction, spatial distribution and size dispersion are examined. Lots of simulations have been carried out using a fast multipole-accelerated boundary element method, and the results are compared to some of the existing models. In this work, all of the results were obtained by using 15 terms for the multipole expansion and $10^{-8}$ for the convergence tolerance. A problem with $5 \times 10^{5}$ unknowns typically requires $8 \sim 10$ hours to run on a Linux node equipped with Pentium IV CPU. 
Three cases are studied: (I) monodisperse flakes, (II) flakes with a bimodal size dispersion and (III) flakes with a normal size dispersion. In case (I), two flake aspect ratios ( $\alpha=5$ and $\alpha=50)$ are studied. In case (II), a mixture of many small flakes ( $\alpha=5)$ and a few large flakes $(\alpha=50)$ is assumed, and the mass fraction of small flakes and that of large flakes are made equal. In case (III), two normal distributions are studied. They are: $\mu(\alpha)=5, \sigma(\alpha)=1$ and $\mu(\alpha)=50, \sigma(\alpha)=10$. For each case, the effect of flake volume fraction $\phi$ is studied at three levels: $0.05,0.10$ and 0.15 . To take account for microstructural variations, ten RVE samples $\left(n_{r}=10\right)$ were generated to form one simulation group subject to the same constraints $\left(\phi, \alpha, N_{f}\right)$, and each REV contains $N_{p} \geq 500$ flakes. The parameter settings are summarized in Table 1 . In all, there are 15 simulation groups and a total of 150 simulations. The mean $\mu$ and the standard deviation $\sigma$ of $\bar{P}$ are calculated.

Table 3. Parameters for cases of study.

\begin{tabular}{|c|c|c|c|c|}
\hline Case & $\alpha$ characteristics & $\phi$ & $N_{f}$ & $n_{r}$ \\
\hline $\begin{array}{c}\text { (I) } \\
\text { monodisperse }\end{array}$ & $\begin{array}{c}5 \\
50\end{array}$ & $\begin{array}{l}0.05 \\
0.10 \\
0.15\end{array}$ & 500 & 10 \\
\hline $\begin{array}{c}\text { (II) } \\
\text { bimodal }\end{array}$ & $\begin{array}{l}50 \text { vol\% }(\alpha=5)+ \\
50 \text { vol\% }(\alpha=50)\end{array}$ & $\begin{array}{l}0.05 \\
0.10 \\
0.15\end{array}$ & 550 & 10 \\
\hline $\begin{array}{c}\text { (III) } \\
\text { Gaussian }\end{array}$ & $\begin{array}{c}\mu(\alpha)=5, \sigma(\alpha)=1 \\
\mu(\alpha)=50, \sigma(\alpha)= \\
10\end{array}$ & $\begin{array}{l}0.05 \\
0.10 \\
0.15\end{array}$ & 500 & 10 \\
\hline
\end{tabular}

The improvement of barrier performance by adding impermeable flakes is usually measured by the relative permeability $P_{0} / P_{f}$, which is defined as the ratio of the permeability of the original membrane to the permeability of the flake-filled membrane. The following discussion of the numerical results are therefore in terms of $P_{0} / P_{f}$.

For case (I), the numerical results are compared to a model proposed by Cussler et al. [1988, 2004]:

$$
\frac{P_{0}}{P_{f}}=\frac{\left(1+\frac{1}{3} \alpha \phi\right)^{2}}{1-\phi}
$$


as well as to Nielsen’s tortuosity model [1967]:

$$
\frac{P_{0}}{P_{f}}=\frac{1+\frac{\alpha}{2} \phi}{1-\phi}
$$

The comparison is made by plotting pairs with predictions from Eq. (3) as $x$ coordinates. Hence in Fig. 7, the solid line through the origin with a unit slope represents Eq. (3) and the dashed line represents Eq. (4). Our numerical results are in general higher than those predicted by both Eq. (3) and Eq. (4), and they are closer to the former than to the latter. In the non-dilute regime ( $\alpha \phi \geq 1)$, Nielsen's model is known to underestimate $P_{0} / P_{f}$ as it does not take into account the horizontal variations in concentration of the solute when it goes around an obstacle. Based on the comparison with our numerical results, Eq. (3) is better, particularly at large $(\alpha \phi)$ levels.

Polydisperse flakes are always encountered in real applications; hence case (II) and case (III) are of more practical interest. Lape et al. [2004] showed that the relative permeability of membranes filled with polydisperse flakes can be predicted by replacing the term $(1 / 3 \alpha \phi)$ in Eq. (3) with a weight-average (or equivalently a volume-average), which results in:

$$
\frac{P_{0}}{P_{f}}=\frac{1}{1-\phi_{\text {tot }}}\left[1+\left(\frac{1}{3} \frac{\phi_{\text {tot }}}{t \sum_{i} n_{i} l_{i}}\right) \sum_{i} n_{i} l_{i}^{2}\right]^{2}
$$

for flakes assuming a discrete size dispersion and

$$
\frac{P_{0}}{P_{f}}=\frac{1}{1-\phi_{t o t}}\left[1+\left(\frac{1}{3} \frac{\phi_{t o t}}{t \bar{l}}\right) \int_{0}^{\infty} l^{2} g(l) d l\right]^{2}
$$

for flakes assuming a continuous size dispersion. In Eq. (5), the subscript $i$ is used to indicate different size classes; $n_{i}$ and $l_{\mathrm{i}}$ are the number and the size of flakes in the $i^{\text {th }}$ size class, respectively; $\phi_{\text {tot }}$ is the total flake volume fraction. In Eq. (6), $g(l)$ is the probability distribution function of flake sizes.

It is natural for us to ask if the weight-average can be replaced by number-average. The latter results in 


$$
\frac{P_{0}}{P_{f}}=\frac{1}{1-\phi_{\text {tot }}}\left[1+\left(\frac{1}{3} \frac{\phi_{\text {tot }}}{t \sum_{i} n_{i}}\right) \sum_{i} n_{i} l_{i}\right]^{2}
$$

for flakes assuming a discrete size dispersion and

$$
\frac{P_{0}}{P_{f}}=\frac{1}{1-\phi_{\text {tot }}}\left[1+\left(\frac{1}{3} \frac{\phi_{\text {tot }}}{t}\right) \int_{0}^{\infty} g(l) l d l\right]^{2}
$$

for flakes assuming a continuous size dispersion.

In Fig. 8, the numerical results for case (II) are compared to the predictions from Eq. (5) and Eq. (7). At all three $\phi$ levels, our numerical results show barrier improvement factors higher than those can be predicted from Eq. (5) and Eq. (7). Apparently, Eq. (7), which is base on the number-average, largely underestimates the barrier performance under the studied conditions, while Eq. (5) is in close agreement with the numerical results.

In Figure 9, the numerical results for case (III) are compared to the predictions from Eq. (6) and Eq. (8). Again, the numerical results are consistently higher than those predicted by models, and the number-average model predicts barrier improvement factor lower than the weight-average model. It is observed that the difference between the numberaverage model and the weight-average model diminishes when the dispersion $(\sigma(\alpha))$ in flake size is small. However, when the dispersion is large and $\alpha$ is large, it is showed again that the weight-average model is a better candidate than the number-average model.

\section{References}

L.E. Nielsen, Models for the permeability of filled polymer systems. J. Macromol. Sci. Chem. A1, 929 (1967).

E.L. Cussler, S.E. Hughes, W.J. Ward, R. Aris, Barrier Membranes. J. Membrane Sci., 38, 161 (1988).

N.K. Lape, E.E. Nuxoll, E.L. Cussler, Polydisperse flakes in barrier films. J. Membrane Sci., 236, 29 (2004). 


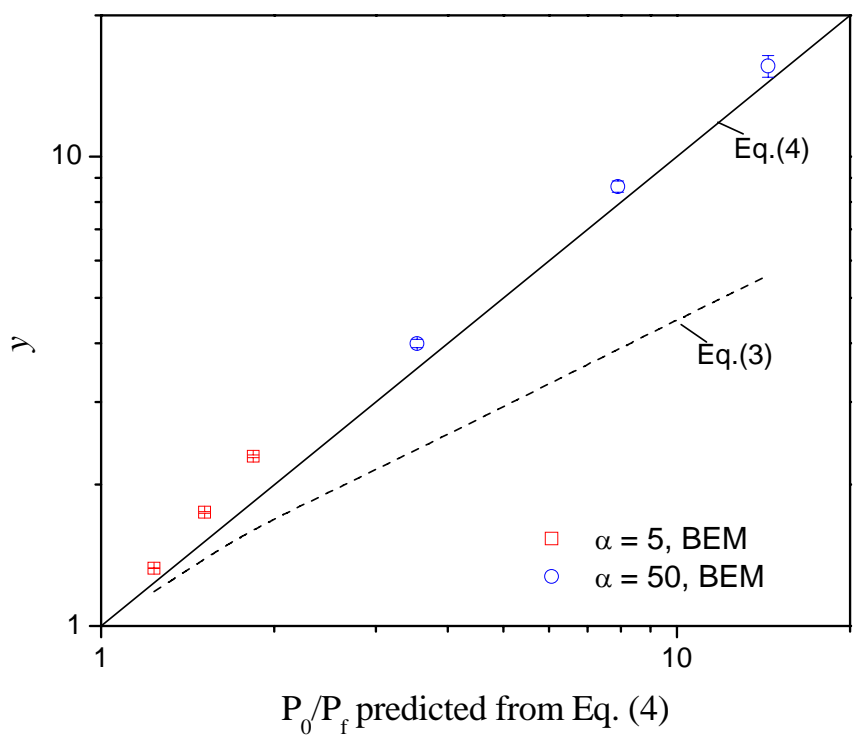

Fig. 7. Comparison of numerical results for case (I) with those predicted by Eq. (3) and Eq. (4).



Fig. 8. Comparison of numerical results for case (II) with those predicted by Eq.(5) and Eq. (7). The error bars indicate the standard deviations in numerical results. 

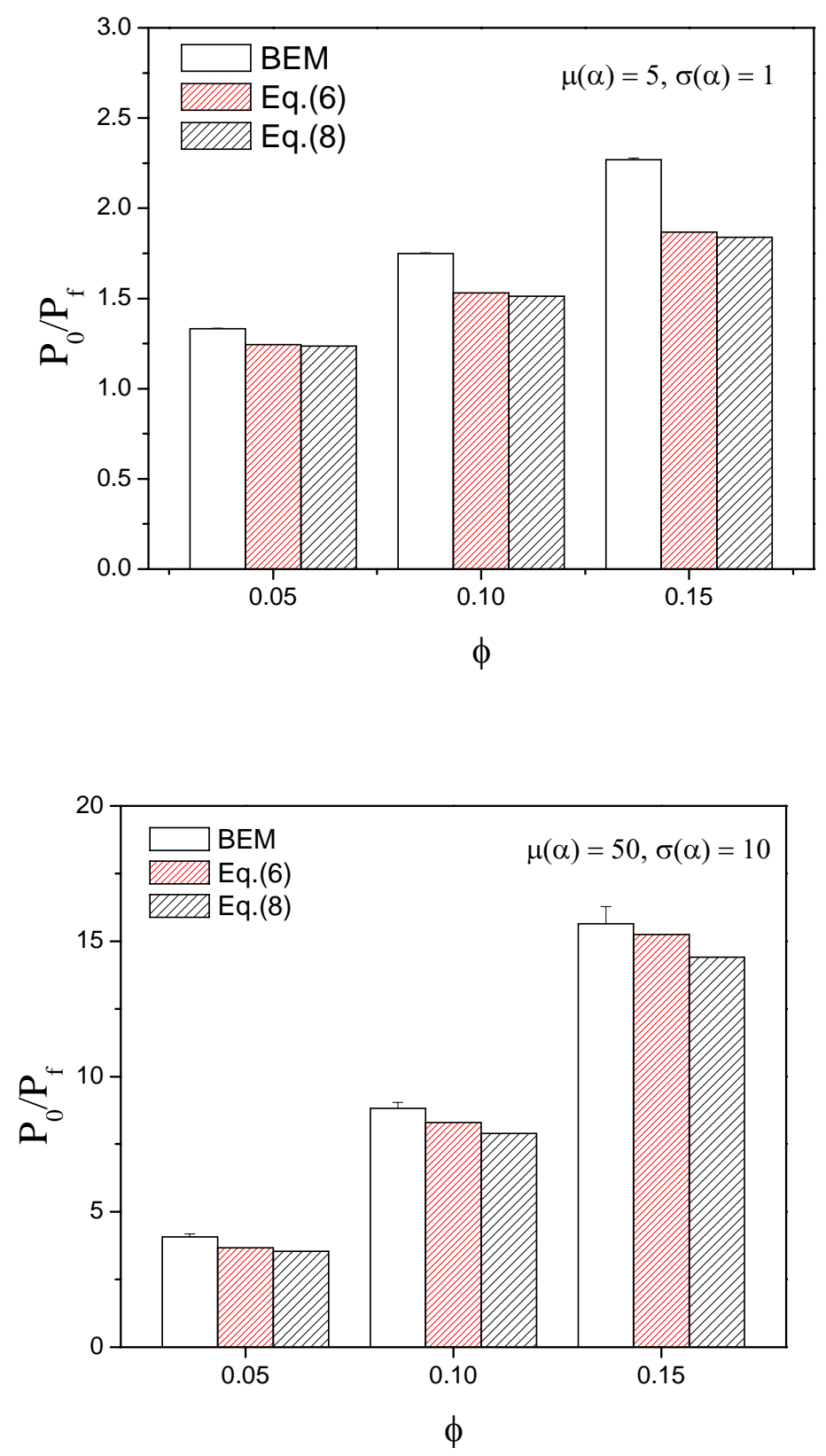

Fig. 9. Comparison of numerical for case (III) with those predicted by Eq. (6) and Eq. (8). The error bars indicate the standard deviations in numerical results. 
Activities Report submitted

for project:

"A hierarchical, structure-oriented and stochastic approach to model liquid molding processes"

Award Number:

DMI-0522221

PI:

Thanasis D. Papathanasiou

Department of Chemical Engineering

University of South Carolina

Columbia, SC 29208

\section{Summary}

Our research activities in the previous year were focused on the following:

(1) Continued to develop codes that simulate microstructure, and to test/propose metrics that quantify microstructural features such as fiber clustering.

(2) Continued to investigate the effects of microstructure on the permeability of unidirectional fibrous media, using the previously developed 2D parallel boundary element codes. Specifically, the effects of non-uniform inter-tow and intra-tow fiber packing and random fiber clustering were studied.

(3) Started to implement a parallel boundary element code for solving threedimensional Stokes flow problems.

(4) Started to implement the Fast Multipole Boundary Element Method. The FMBEM for solving two-dimensional potential flow problems has been applied to study the barrier improvement factor in flake-filled membranes.

Attended

(1) Workshop on 'A short course on the Fast Multipole Boundary Element Method', Los Angeles, California, USA. July 22, 2006.

(2) ANTEC 2007, Cincinnati, Ohio, USA. May 7-11, 2007. 


\section{Microstructure Models}

\subsection{Perturbed Dual-scale Structures}

In resin transfer molding, fibers are often bundled in order to ease handling as well as to facilitate the mold filling process. In previous studies, ordered dual-scale structures in which both intra-tow and inter-tow packing show similar orderly features have been used to model assemblies of fiber bundles. Such ordered dual-scale models are characterized by a number of parameters, namely the inter-tow porosity $\phi_{\mathrm{s}}$, the intra-tow porosity $\phi_{\mathrm{t}}$ and fiber tow dimensions [Sadiq et al., 1995; Papathanasiou 2001]. It is noted that $\phi_{\mathrm{s}}$ and $\phi_{\mathrm{t}}$ do not depend on the local fiber packing.

In this work, stochastic features were introduced to a dual-scale model for arrays of fiber clusters, namely, non-uniform intra-tow packing and non-uniform inter-tow packing. The non-uniform intra-tow packing can be generated by a hard-core model [Allen and Tildesly, 1987] with varied minimum inter-fiber spacing $\delta_{\min }$. It has been shown [Chen and Papathanasiou, 2006] that the degree of heterogeneity in a hard-core fiber packing can be manipulated by proper choice of $\delta_{\min }$. The larger the degree of heterogeneity is, the smaller $\delta_{\min }$ should be (Fig.1).

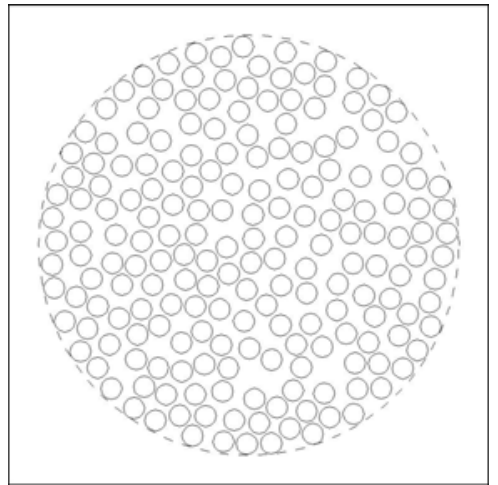

(a)

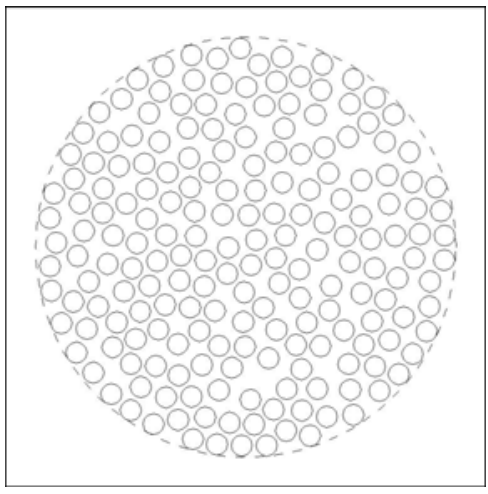

(b)

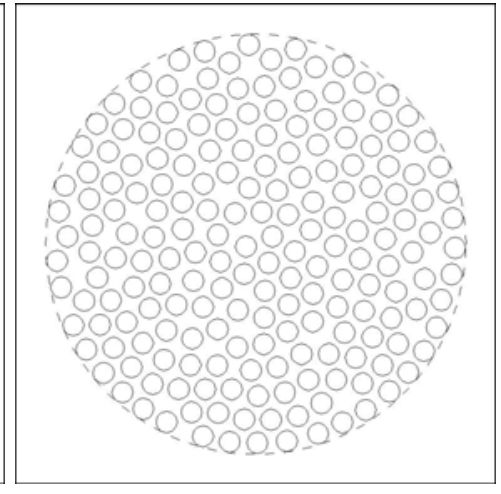

(c)

Fig.1. Effect of $\delta_{\min }$ on the intra-tow fiber packing. (a) $\delta_{\min }=0.05 D_{\mathrm{f}},(b) \delta_{\min }=0.10 D_{\mathrm{f}}$, (c) $\delta_{\min }=0.25 D_{\mathrm{f}}$.

In the case of non-uniform inter-tow packing, two perturbation modes were introduced to a square packing of fiber tows. First, the horizontal spacing between fiber tow columns was perturbed, with the vertical spacing between rows of fiber tows remaining fixed 
(Fig.2(a)). Second, the vertical spacing between rows of fiber tows was perturbed, with the horizontal spacing between fiber tow columns remaining fixed (Fig.2(b)). The extent of horizontal perturbation can be characterized by a minimum inter-tow spacing $\Delta_{\min }$. The extent of vertical perturbation can be characterized by maximum vertical shift $\Lambda_{\max }$. The larger the extent of perturbation, the smaller $\Delta_{\min }$ or the larger $\Lambda_{\max }$ should be.
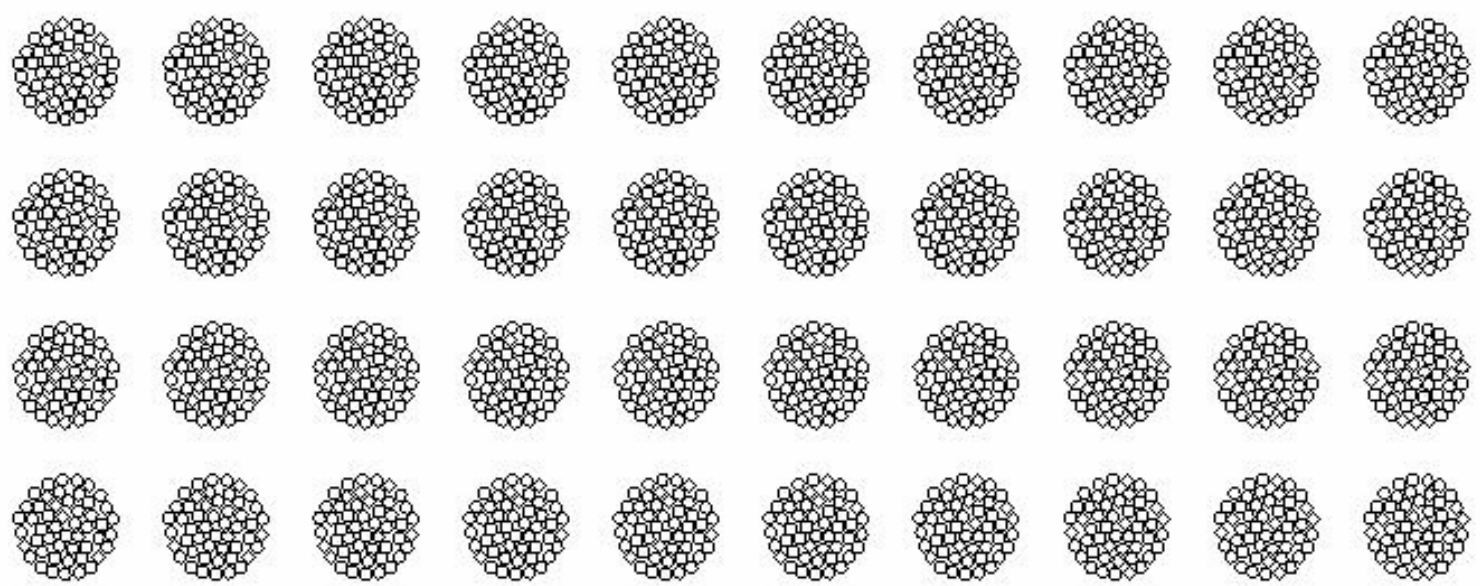

(a)
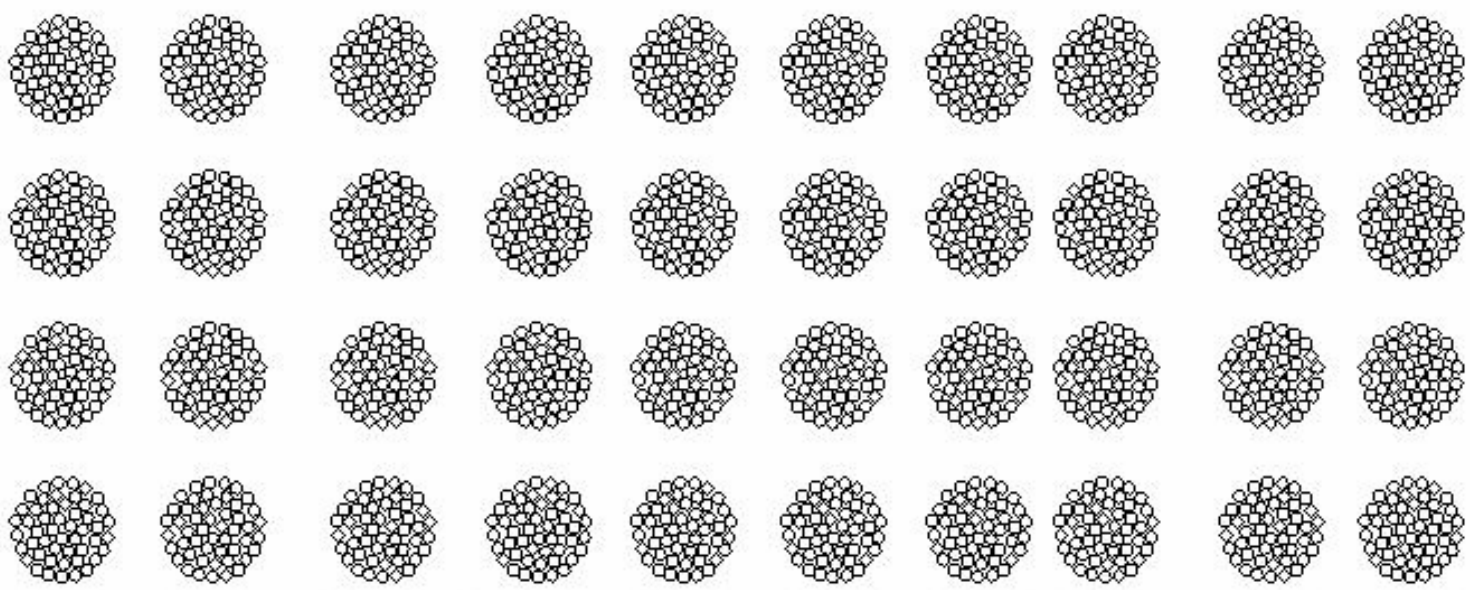

(b) 


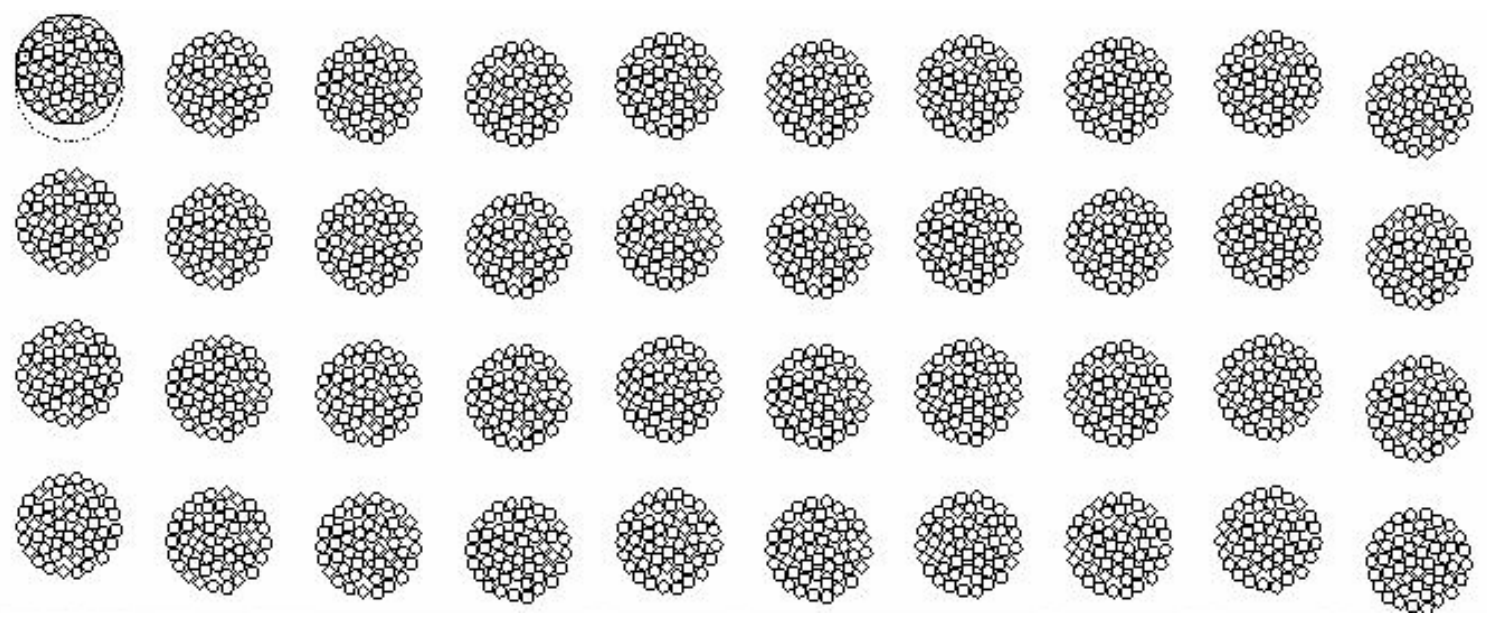

Fig.2. Schematic illustration of perturbation modes introduced to a square packing of fiber tows. (a) original, (b) horizontally perturbed, (c) vertically perturbed.

\subsection{Random Fiber Clustering}

Fiber clustering manifests itself in various forms. A man-made fiber tow is only one form of fiber clustering. In actual processing conditions, fiber tows may loosen their shape when they are under external forces. Fiber clustering is understood as a result of a balance between the attractive force that tends to hold fibers together and the repulsive force that tends to separate fibers. Clustered fiber distributions can be generated with a NVT Monte Carlo method [Allen and Tidesley, 1987], a process during which the minimization of the potential energy of the system is sought. In this Monte Carlo process, fibers are moved from their initial location; the change in the system potential energy is calculated for each attempted move; any downhill move in energy will be accepted and any uphill move in energy will be accepted with a probability of $\exp \left(-\Delta E / k_{B} T\right), T$ being temperature, $\Delta E$ being the energy change and $k_{\mathrm{B}}$ being the Boltzmann constant. Here the physical interpretation of 'temperature' depends on what type of real physical process is under discussion. The definition of the dimensionless temperature $\left(T^{*}\right)$ is: $T^{*}=k_{B} T / \varepsilon$, indicating the relative importance of kinetic energy to potential energy. To carry out the Monte Carlo process, an explicit form of pair potential that describes the repulsive and attractive forces between a pair of fibers must be provided in order to compute the system potential energy. The simplest potential model that includes both forces is the LennardJones potential. Accounting for the fact that fibers are not penetrable, a modified 
Lennard-Jones potential was used, in which the hard-disk potential at distances $r \leq r_{\sigma}$ replaces its usual behavior.

$$
v(r)=\left\{\begin{array}{cc}
4 \varepsilon\left[\left(\frac{r_{\sigma}}{r}\right)^{12}-\left(\frac{r_{\sigma}}{r}\right)^{6}\right] & \leq r_{\sigma} \\
0 & <r<r_{c} \\
r_{\sigma} & >r_{c}
\end{array}\right.
$$

In Eq. (1), $v$ is the pair potential, $\varepsilon$ is the depth of the potential well, $r_{\sigma}$ is the finite distance at which the potential becomes positive and $r_{\mathrm{c}}$ is the cutoff distance after which the tail part of the potential is neglected.

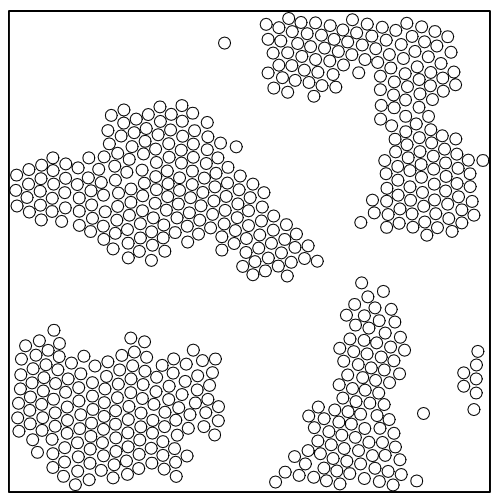

(a)

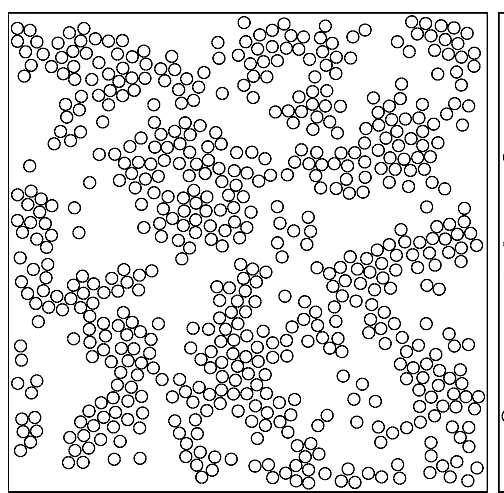

(b)

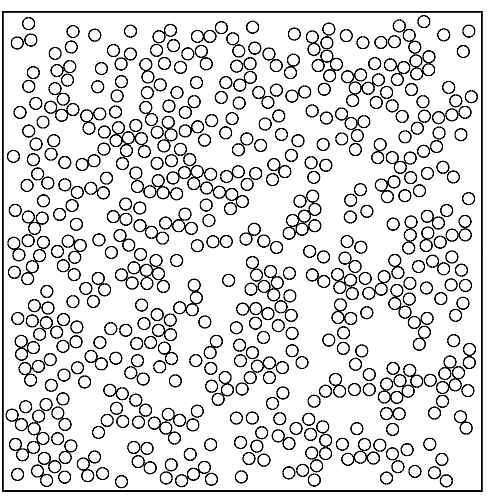

(c)

Fig. 3. Sample distributions generated by the NVT Monte Carlo method with $N_{\mathrm{f}}=576, \phi=0.7, \delta_{\min } / D_{\mathrm{f}}=0.05$. (a) $T^{*}=0.3$, (b) $T^{*}=0.5$ (c) $T^{*}=1.0$. The extent of fiber aggregation is decreased as the dimensionless temperature $\left(T^{*}=k_{\mathrm{B}} T / \varepsilon\right)$ is increased.

In Fig.3, $\varepsilon$ and $r_{\sigma}$ are fixed, hence the generated fiber distributions are affected by $T^{*}$ only. The extent of fiber clustering is inversely related to $T^{*}$.

\section{Microstructure Characterization}

To develop microstructure-permeability correlations, microstructural characterization is as important as flow modeling. This naturally poses the question of the choice of a "suitable" metric that will correlate a microstructure to the corresponding permeability. This issue is far from resolved today, even though several spatial metrics have been used to characterize fiber distributions. Pyrz [1994] applied the nearest neighbor distance function, the second-order intensity function and the radial distribution function to 
investigate the relation between the morphology and the stress distribution in unidirectional fiber composites. Pearce et al. [2000] determined the fractal dimension in fabric-reinforced composites and reported a correlation between the fractal dimension and permeability. Bechtold and Ye [2003] used the Morishita index to characterize the distribution of filaments inside fiber bundles and found that the local permeability decreased with an increase in the value of the Morishita index. The so-called mean free path was used by Bizet et al. [2004] to analyze the microstructure of yarns in a non-crimp stitched carbon/polyester composite.

In this section, the Ripley's K function was explored to distinguish between different fiber distributions. Based on the evaluations of Ripley's K function, a metric called the measure of deviation was proposed to quantify the degree of fiber clustering. This is based on the averaging of the ratios between the $K(r)$ of a given microstructure and $\pi r^{2}$, the $K(\mathrm{r})$ of a Poisson distribution. The definition of the measure of deviation is:

$$
\chi=\frac{1}{b-a} \int_{a}^{b} \frac{K(r)}{\pi r^{2}} d r \approx \frac{1}{m} \sum_{i=1}^{m} \frac{K\left(r_{i}\right)}{\pi r_{i}^{2}}
$$

where $[a, b]$ is the length scale range of interest. Usually, $a$ is the fiber diameter, and $b$ has to be larger than the largest fiber cluster size. The measure $\chi$ has been applied to quantify the clustered fiber distributions which are generated by the NVT Monte Carlo method.

\section{Parallel Implementation of a 3D BEM Code}

Having a three-dimensional BEM code will extend our ability to modeling threedimensional fiber preforms. This part of work is to expand the library in our twodimensional BEM code to include the treatment of surface integrals. A sequential 3D BEM code was implemented first and benchmarked. However, the bottlenecks in carrying out sequential 3D BEM computations still lie in the memory and CPU requirements. To overcome these limitations, a parallel implementation of the 3D BEM code was sought. Parallelization of the BEM is not a new topic. Our practice below follows Natarajan and Krishnaswamy [1995]. 
The target parallel computer is a commodity cluster, which has a distributed-memory architecture. Such a commodity cluster, typically consisting of several dozens of nodes (each equipped with dual CPUs, RAM and disk) and a fast Ethernet network, has become common in scientific computing groups. The programming environment typically consists of a Linux operating system, a Fortran compiler, a Message Passing Interface [Gropp and Lusk, 1994] library to handle inter-processor communications, and other parallel libraries for specific purposes. A parallel program can be written in the SingleProgram Multiple-Data programming model.

From the implementation point of view, the two major compute-intensive steps in the BEM are the assembly phase for generating the entries of the coefficient matrix $\mathbf{A}$ and the right hand side vector $\mathbf{b}$, and the solver phase for solving the resulting linear system of equations. The overall approach that we use is to replace the solver with the dense-matrix solver routines from the ScaLAPACK [Blackford et al., 1997] library and to do significant code modifications in other phases of the BEM for parallel performance and efficiency. Because the best performance using the ScaLAPACK routines is obtained when the matrices are distributed among nodes in a specific format, namely, 2D cyclic block distribution, which is not the natural decomposition for the matrix generation of the BEM, significant modifications are required. While the parallel performance in the solver phase is good, some new parallel overheads may be introduced in the other parts of the code.

The matrix generation part of the BEM can be parallelized with the so-called 'OwnerComputes' algorithm, in which each processor only performs the integration for the matrix entries that are to be stored in it. This algorithm does not require inter-processor communications, therefore the matrix generation is performed without parallel overheads. However, most of the work in the matrix entry evaluation consists of computing the isoparametric transformation, and evaluating the integrand at the quadrature points, and there is little difference in the amount of work to compute a single entry or all entries of the element matrix. It is evident that the 'Owner-Computes' algorithm will introduce 
redundant computations because entries of the element matrix are distributed over different processors. The other parallelization strategy is to distribute the element matrices among processors, perform the entry evaluations locally, and then transfer the entries to their destined processors according to the 2D cyclic block format. A parallel overhead is introduced in this algorithm as it involves inter-processor communications. However, as such inter-processor communications can be done in a non-blocking way. The parallel overhead involved is quite negligible.

The performance results of the two algorithms have been compared in this work.

\section{Fast Multipole Boundary Element Method}

If there are $N$ unknowns in a discretized system, the conventional BEM will produce an $N x N$ full matrix, whose construction will require operations of complexity proportional to $N^{2}$. By contrast, a domain method such as the Finite Element Method (FEM) only requires $\mathrm{O}(N)$ operations thanks to its banded matrix structure. Even though one may argue that there will be fewer unknowns in the BEM than in the FEM for the same problem, the conventional BEM is considered expensive for large problems. However, recent developments of the fast BEMs have revealed that the discrietized equations in the BEM can be solved with $\mathrm{O}(N)$ operations with the help of the Fast Multipole Method (FMM) [Mammoli and Ingber, 1999; Nishimura, 2002; Liu et al., 2005]. As a result, very fine discretization can now be used in large problems, thus the high accuracy of the BEM can be really enjoyed.

In the previous year, we attended a workshop on the Fast Multipole Boundary Element Method, held in Los Angeles, CA, on July 22, 2006. Based on a tutorial code obtained from the workshop, we have started implementing codes (pre-processor, solver and postprocessor) for the purpose of predicting macroscopic properties of two-phase composite materials, e.g., conductivity, permeability and elastic modulus. The problems we aim to solve are potential flow, Stokes flow and elastostatic problems. We have applied the FMBEM code to predict the barrier improvement factor of flake-filled membranes [Chen and Papathanasiou, 2007]. 
Fig.4. shows a Representative Element Volume (REV) for modeling the one-dimensional diffusion in the thickness direction of a membrane filled with random, aligned flakes. Such REVs were generated in computer using the random sequential addition algorithm. The assumptions about the modeling geometry are:

- The largest dimension of a flake is infinite. The model is two-dimensional, representing a section of the membrane through its thickness.

- In such a two-dimensional plane, the flake cross-sections have rectangular shape characterized by their aspect ratio $(\alpha)$ defined as the ratio of the flake's larger dimension (length $l$ ) to its smaller dimension (thickness $t$ ).

- The flakes are aligned perfectly in the direction normal to the direction of solute diffusion.

- $\quad$ The flakes are randomly aligned.

Regarding this REV, we consider the diffusion of a solute in the thickness direction. The flakes are randomly distributed. It is assumed:

- The membrane phase is homogeneous and isotropic.

- The diffusion coefficient of the solute in the membrane phase is constant, i.e., independent of solute concentration and not affected by the presence of flakes.

- The flake phase is impermeable.

- There are no reaction sites (sources or sinks) in the membrane. 


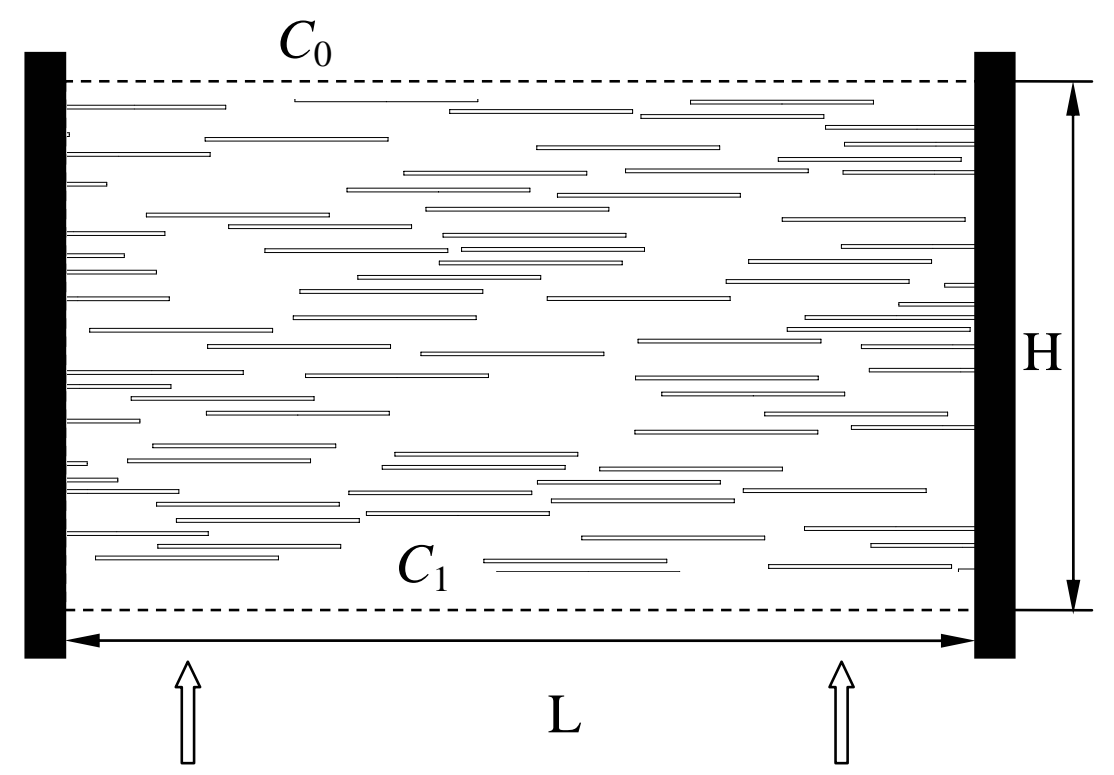

Fig.4. REV of a section of membrane filled with random, aligned flakes.

To compute the effective permeability $\bar{P}$ of the system described in the above, we let the solute concentration at the bottom surface of the membrane be fixed at $C_{1}$, the solute concentration at the top surface of the membrane be fixed at $C_{0}$, and assume there is no lateral diffusion. The effective permeability in the thickness direction can be calculated by

$$
\bar{P}=\frac{\bar{J}}{\left(C_{1}-C_{0}\right) / H}
$$

In Eq. (3), $\bar{J}$ is the overall permeate flux in the thickness direction ( $y$-axis), which is to be found by averaging the local fluxes along the boundary as obtained from the numerical solution.

\section{References:}

Allen M.P. and Tidesley D.J., Computer Simulation of Liquids, Oxford University Press, New York (1987). 
Bechtold, G. and Ye, L. Influence of fiber distribution on the transverse flow permeability in fiber bundles. Comp. Sci. Technol. 63:2069-2079 (2003).

Bizet, L., Breard, J. Bouquet, G., Jernot, J-P. and Gomina, M. Serial sections through a continuous fiber-reinforced polymer composite. Image Anal. Stereol. 23:167-176 (2004).

Blackford, L.S., Choi, J., Cleary, A., D'Azevedo, E., Demmel, J., Dhilllon, I., Dongarra, J., Hammarling, S., Henry, G., Stanley, K., Walker, D., Whaley, R.C.: ScaLAPACK user's guide. SIAM, Philadelphia (1997)

Chen X, Papathanasiou T.D., On the variability of the Kozeny constant for saturated flow across unidirectional disordered fiber arrays. Composite Part A 37: 836-846 (2006).

Chen X, Papathanasiou T.D., Permeability of Flake-filled Barrier Membranes: A Numerical Evaluation, ANTEC Paper \#0621, Cincinnati, OH, USA, 2007.

Gropp, W., Lusk, E: Using MPI: portable parallel programming with the message passing interface. MIT press (1994)

Liu Y.J. and N. Nishimura, The fast multipole boundary element method for potential problems: a tutorial, Eng. Anal. Bound. Elem. 30: 371-381 (2006).

Liu Y.J., Nishimura N, Otani Y, Takahashi T, Chen X.L., Munakata H. A fast boundary element method for the analysis of fiber-reinforced composites based on a rigid-inclusion model. J Appl Mech 72(1): 115-28 (2005).

Mammoli A.A. and Ingber M.S., Stokes flow around cylinders in a bounded twodimensional domain using multipole-accelerated boundary element methods, Int. J. Numer. Methods Eng., 44, 897-917 (1999).

Natarajan R. and Krishnaswamy D. A case study in parallel scientific computing: the boundary element method on a distributed-memory multicomputer. SC'95, San Diego, CA.

Nishimura N. Fast multipole accelerated boundary integral equation methods. Appl Mech Rev 55(4 ):299-324 (2002).

Papathanasiou, T.D. Flow across structured fiber bundles: a dimensionless correlation. Int. J. Multiphase Flow 27:1451-1461 (2001).

Pearce, N.R.L., Summerscales, J. and Guild, F.J. Improving the resin transfer molding for fiber reinforced composites by modification of the fabric architecture. Compos. Part A. 31:1433-1441 (2000).

Pyrz, R. Quantitative description of the microstructure of composites: I. Morphology of unidirectional composite systems. Comp. Sci. Technol. 50:197-208 (1994). 
Sadiq, T.A.K., Advani, S.G. and Parnas, R.S. Experimental investigation of transverse flow through aligned cylinders. Int. J. Multiphase Flow 21(5):755-774 (1995). 
Annual Report for Period:09/2008 - 08/2009

Principal Investigator: Burghardt, Wesley R.

Organization: Northwestern University

\section{Submitted By:}

Burghardt, Wesley - Principal Investigator

Title:

Collaborative Research: Microstructural Modeling and Synchrotron Studies of Orientation Development in Injection Molding of Liquid Crystalline Polymers

\section{Senior Personnel}

Name: Burghardt, Wesley

Worked for more than 160 Hours: Yes

Contribution to Project:
Submitted on: 12/07/2009

Award ID: 0521823

\section{Project Participants \\ Project Participants}

\section{Post-doc}

\section{Graduate Student}

Name: Fang, Jun

Worked for more than 160 Hours: Yes

\section{Contribution to Project:}

Mr. Fang is the graduate student currently supported on this grant. His efforts include preparing \& performing experiments, analyzing data, and performing simulations.

\section{Undergraduate Student}

Name: Dougherty, Leah

Worked for more than 160 Hours: No

Contribution to Project:

Leah performed rheological experiments on model polymer nanocomposite materials. She did this work as an undergraduate resarch/independent study project, for academic credit.

\section{Technician, Programmer}

\section{Other Participant}

\section{Research Experience for Undergraduates}

\section{Michigan Molecular Institute}

\section{Organizational Partners}

This is a collaborative project with Dr. Robert Bubeck from Michigan Molecular Institute. Dr.

Bubeck takes the lead on surface orientation measurements using x-ray spectroscopy; $\mathrm{Mr}$.

Fang from Northwestern has participated in experiments of this sort led by Dr. Bubeck.

Conversely, Dr. Bubeck has also participated in several synchrotron x-ray scattering

experiments led by our group at Northwestern.

\section{Saginaw Valley State University}


We continue collaborative efforts with Professor Bruce Hart who, along with SVSU undergraduate students, assists with mechanical property charactization of TLCP moldings.

Other Collaborators or Contacts

We have had contact with engineers from MoldFlow corporation, who have helped address questions related to the modeling portion of this project.

\section{$\underline{\text { Activities and Findings }}$}

\section{Research and Education Activities:}

During 9/1/08 ? 8/31/09 we (i) completed a coordinated experimental/computational study of surface orientation in LCP moldings, (ii) pursued time-dependent simulations of TCLP orientation during injection molding in order to draw comparisons to existing in situ time-resolved data, and (iii) initiated a final series of experiments to study time-evolution of orientation state during molding via ?short shot? moldings.

Our collaborative effort with Dr. Bubeck in the area of synchrotron-based NEXAFS and lab-based IR spectroscopy studies of surface orientation led to submission of a manuscript (currently in press). In this study, spectroscopic measurements of surface orientation are compared to x-ray scattering measurements of bulk orientation in TLCP moldings. Data were compared to process simulations of molecular orientation using the Larson-Doi polydomain model, following the fiber analogy that underlies the computational program in this project. The model is capably of qualitatively describing many fine points of both bulk and surface orientation distributions.

The major effort during 08-09 was completion of extensive time-resolved simulations of molecular orientation during mold filling, and comparison of these simulations against in situ time-dependent orientation data obtained using synchrotron x-ray scattering in earlier years. Preparation of a manuscript on this effort, which is the centerpiece result of this project, is underway.

Finally, motivated in part by some discrepancies between simulations and experiments in our ex situ study of bulk and surface orientation in TLCP moldings, we performed x-ray scattering experiments to measure bulk orientation distribution in ?short shot? moldings that are incompletely filled. These data will be used to track evolution of 2-D orientation distributions during molding, and also be used to test simulations of orientation designed to mimic the short-shot molding conditions.

Beyond the primary purpose of the project as the focal point in Mr. Fang?s graduate education, results were also disseminated to the broader community via two conference presentations during 2008-09 at the American Physical Society meeting, focusing on (i) simulation and experiments on bulk vs surface orientation, and (ii) simulation of time- dependent injection molding. Professor Burghardt also spoke on this project in a Keynote Lecture delivered at SPE ANTEC 2009, in June in Chicago.

\section{Findings:}

NEXAFS and IR spectroscopies yield qualitatively and quantitatively similar information regarding surface orientation distributions, and confirm that both the degree and direction of molecular orientation in the surface region can differ substantially from that in the bulk of injection molded plaques. Larson-Doi polydomain qualitatively capture trends in the differences between surface and bulk orientation. 
Time-resolved experiments on three different commercial TLCPs in the same square- and T-shaped plaque molds yield broadly similar distributions of molecular orientation, suggesting that TLCPs exhibit similar orientation dynamics during processing, independent of chemical identity. Comparisons between time-resolved simulations and in situ transient WAXS orientation data collected during molding reveals a discrepancy in that experiments show orientation to continue to evolve following completion of mold filling, which simulations do not capture. However, simulations perform reasonably well in predicting the final bulk orientation distribution in liquid crystalline polymer moldings.

\section{Training and Development:}

Mr. Fang is poised to defend his PhD thesis during 2009-10. On the strength of his research accomplishments working on this project, Mr. Fang was awarded a competitive ?terminal year? fellowship at Northwestern.

\section{Outreach Activities:}

Burghardt delivered a Keynote Lecture describing this project at SPE ANTEC 2009, held in conjunction with the NPE plastics trade show in Chicago, in June 2009.

\section{Journal Publications}

Rendon, S.; Burghardt, W. R.; Bubeck, R.A., "Orientation Dynamics in Commercial Thermotropic Liquid Crystalline Polymers in Transient Shear Flows", Rheologica Acta, p. 945, vol. 46, (2007). Published,

Rendon, S.; Bubeck, R. A.; Thomas, L. S.; Burghardt, W.R.; Hexemer, A.; Fischer, D. A., "Interrogation of 'Surface', 'Skin' and 'Core' Orientation in Thermotropic Liquid Crystalline Copolyester Moldings by Near-Edge X-ray Absorption Fine Structure and Wide-Angle X-ray Scattering", Journal of Applied Polymer Science, p. 2502, vol. 106, (2007). Published,

Rendon, S.; Burghardt, W. R.; Auad, M. L.; Kornfield, J. A., "Shear-Induced Alignment of Smectic Side Group Liquid Crystalline Polymers", Macromolecules, p. 6624, vol. 40, (2007). Published,

J. Fang, W. R. Burghardt and R. A. Bubeck, "Polydomain Model Predictions of Molecular Orienttation in Isothermal Channel Flows of Thermotropic Liquid Crystalline Polymers", Polymer Engineering and Science, p. 22, vol. 48, (2008). Published,

S. Rendon; J. Fang; W. R. Burghardt; R. A. Bubeck, "An Apparatus for In Situ X-ray Scattering Measurements During Polymer Injection Molding", Rev. Sci. Instrum., p. 043902, vol. 80, (2009). Published,

J. Fang; W. R. Burghardt, "In situ X-ray scattering measurements and polydomain simulations of molecular orientation development during injection molding of liquid crystalline polymers", AIP Conference Proceedings, p. 39, vol. 1027, (2008). Published,

\section{Books or Other One-time Publications}

\section{Web/Internet Site}

\section{Other Specific Products}

\section{Contributions within Discipline:}

\section{Contributions}

We have performed the first realistic simulations of molecular orientation of liquid crystalline 
polymers during injection molding, using the polydomain model of Larson and Doi. Simulated orientation distributions have been compared to: (i) ex situ measurements of bulk orientation distributions obtained via x-ray scattering; (ii) ex situ measurements of surface orientation distributions obtained via NEXAFS and FTIR spectroscopies; and (iii) in situ, timeresolved measurements of molecular orientation development obtained via synchrotronbased scattering methods.

\section{Contributions to Other Disciplines:}

\section{Contributions to Human Resource Development:}

Education of graduate students, undergraduate students and summer visitors; outreach activities led from MMI to support research in a primarily undergraduate institution.

\section{Contributions to Resources for Research and Education:}

Our continuing development of the experimental infrastracture for studying polymer structure via synchrotron $\mathrm{x}$-ray scattering during injection molding is a significant step forward. As with previous instrumentation we have developed, we plan to make this instumentation available to other investigators upon request.

Contributions Beyond Science and Engineering:

\section{Conference Proceedings}

\section{Special Requirements}

Special reporting requirements: None

Change in Objectives or Scope: None

Animal, Human Subjects, Biohazards: None

Categories for which nothing is reported:

Any Book

Any Web/Internet Site

Any Product

Contributions: To Any Other Disciplines

Contributions: To Any Beyond Science and Engineering

Any Conference 
Final Report for Period: 09/2008 - 08/2009

Principal Investigator: Bubeck, Robert A.

Organization: Michigan Molecular Inst

Submitted By:

Bubeck, Robert - Principal Investigator

Title:

Collaborative Research: Microstructural Modeling and Synchrotron Studies of Orientation Development in Injection Molding of Liquid Crystalline Polymers

\section{Senior Personnel}

Name: Bubeck, Robert

Worked for more than 160 Hours: Yes

Contribution to Project:

Co-PI of the project.

Name: Thomas, Lowell

Worked for more than 160 Hours: No

Contribution to Project:

Lowell Thomas provides in depth expertise in the processing of engineering polymers. He also performs the injection molding of sample plaques and the preconditioning (extruder/devolitalization) of thermotropic liquid crystalline copolyesters studied in the project. Mr. Thomas is a Fellow of the SPE.

\section{Post-doc}

\section{Graduate Student}

Name: Fang, Jun

Worked for more than 160 Hours: No

Contribution to Project:

Jun 'Tony' Fang is a graduate student reporting to Prof. Wesley Burghardt (Northwestern University) and is supported by Prof. Burghardt's Co-PI grant related to our collaborative research. Mr. Fang accompanied me (Bubeck) this past February (2008) to the National Synchrotron Light Source at Brookhaven National Labs. He has written a new macro to automatically translate and rotate azimuthal and incident angles while maintaining fixed incident beam positions with the new 6-axis installed in the experimental station at U7A.

\section{Undergraduate Student}

Name: Fondriest, Heather

Worked for more than 160 Hours: No

Contribution to Project:

Working with Mr. Bruce Hart and myself, Ms. Fondriest provided sample machining and tensile data to the project. she received her B.S. degree in Mechanical Engineering in June.

Name: Burgard, Susan

Worked for more than 160 Hours: No

\section{Contribution to Project:}

3.Susan M. Burgard is a technical cooperative education student employed with Impact Analytical, the characterization arm of MMI. She is an undergraduate biology major with a minor in chemistry at SVSU and is doing a cooperative project directly related to this grant under the direction of myself and Ms. Katherine Robertson (Impact analytical) performing Fourier Transform Infrared Attenuated Total Reflectance (FTIR-ATR) spectroscopy measurements on TLCP samples. The cooperative project is defined to characterize a specific sample set with samples in common with the mechanical and synchrotron-based measurements being made under the grant. Ms. Burgard is now in her senior year at SVSU. 


\section{Technician, Programmer}

Name: Robertson, Katherine

Worked for more than 160 Hours: $\quad$ No

Contribution to Project:

Ms. Katherine Robertson is a Research Chemist for Impact Analytical, the charcaterization arm of MMI, and is responsible for the FTIR instrumentation used in this research and co-supervising Ms. Susan Burgard.

\section{Other Participant}

Name: Fischer, Daniel

Worked for more than 160 Hours: $\quad$ No

Contribution to Project:

Daniel Fischer is the beam line scientist for the NIST/Dow Materials Characterization End Station, Beamline U7A at the National Synchrotron Light Source, Brookhaven National Labs, Upton, NY. Dr. Fischer is a senior staff scientist employed by NIST.

Beamline U7A is the one used for the NEXAFS measurements.

\section{Research Experience for Undergraduates}

\section{Northwestern University}

Organizational Partners

Professor Wesley Burghardt of the Chemical and Biological Engineering Department of Northwestern University is the Co-PI with myself on the project.

\section{Saginaw Valley State University}

Mr. Bruce Hart is the contact and collaborator for specimen machining and physical testing at SVSU. The three undergraduate students who collaborate in the project report to Mr. Hart.

\section{NIST/Brookhaven National Labs}

Beamline U7A is the NIST/Dow Materials Characterization End Station at the National Synchrotron Light Source (NSLS) at Brookhaven National Labs (BNL). Beamline U7A is a soft X-ray spectroscopy facility and is utilized to obtain the near-edge X-ray absorption fine structure (NEXAFS) data for the project. Access for beam time is gained by writing peer-reviewed general user proposals authored by the PI of this project.

\section{Department of Energy Argonne National Laboratory}

The DuPont/Northwestern/Dow Collaborative Access Team (DND-CAT) at the Advanced Photon Source, Argonne National Labs. DND-CAT is a synchrotron-based hard X-ray end station that provides both bending magnet and insertion device X-rays that are used to perform 2-D WAXS on injection-molded plaques and in-situ characterization of TLCP melt flow. Beam time is obtained through a part share of Northwestern University's CAT allotment and by the writing of peer-reviewed general user proposals.

\section{Other Collaborators or Contacts}

Although the bulk of our beam time was granted under our own general user proposal, we also have continued to have an informal collaborative arrangement with Profs. Edward Kramer (U.C.S.B.) and Chris Ober (Cornell University) under which participants from the three universities and M.M.I. share effort and some general user beam time at the National Synchrotron Light Source. Although the technique (NEXAFS) is the same, the materials studied differ considerably, thereby exposing the graduate students and post docs to research concerns regarding engineering polymers to which they might not otherwise normally be exposed.

During the course of evaluating ATR-FTIR-ATR as a tool for the measurement of surface orientation of injection moldings, Dr. Richard Nyquist of Impact Analytical provided both technical input and useful discussions. 


\section{Activities and Findings}

\section{Research and Education Activities: (See PDF version submitted by PI at the end of the report)}

Thermotropic liquid crystalline polymers (TLCPs) are high strength, lightweight engineering materials. Their excellent attributes are intimately linked to the spontaneous ordering of rod-like molecules in the nematic liquid crystalline phase and the subsequent impact of processing on the molecular orientation state. Understanding, manipulating and, ultimately, control over the evolution of molecular orientation during processing is a necessary prerequisite towards optimized fabrication of TLCP net-shaped parts to meet specific material property specifications in the final product. Current studies elucidated links that connect fundamental dynamic properties of nematic materials, through structure evolution in controlled flows, to anisotropic orientation distributions in final injection-molded parts.

At the core of the experimental effort is the development and application of in situ capabilities to perform X-ray scattering measurements of molecular orientation in real time within the injection molding cycle. These experiments have been complemented by further development of a novel ex situ synchrotron spectroscopy technique that offers new capabilities for unprecedented 3-D characterization of the molecular orientation distribution within the surface layer of moldings.

The process of injection molding net-shape parts from thermotropic liquid crystalline polymers (TLCPs) results in a 'skin-core' macrostructure. The underlying orientation in the core and the skin often differs in both magnitude and direction. The influences of molding parameters and mold thickness on the anisotropy and 'skin-core' morphology of injection-molded dihydroxy-alpha-methylstilbene (DHaMS) thermotropic liquid crystalline copolyester were studied in some detail. Duplicate experiments were completed for Ticona Vectra « A950 6-hydroxy-2-naphthoic acid/6-hydroxybenzoic acid (HBA/HNA) type-copolyester.

Comparisons of the surface orientation of the nematic polymers were observed for 2-dimensional injection molded net-shaped parts by using $\mathrm{C}$ K edge NEXAFS and Attenuated Total Reflectance Fourier Transform Infrared (FTIR-ATR) dichroism using a Herrick Seagull variable angle reflectance stage. The contributions of shear and extensional flows on the bimodal orientation character of the moldings were characterized by a combination of 2-D WAXS in transmission, NEXAF, and FTIR-ATR dichroism. The spectroscopies were utilized as a non-invasive in situ means of determining surface layer orientation that probes to a depth of as little as $2 \mathrm{~nm}$ and as great as 5 microns. Orientation parameters for the 'core' regimes were determined using the 2-D WAXS data.

Based upon the knowledge gained, we implemented new modeling strategies to predict orientation development, coordinated with cutting edge synchrotron techniques to characterize the resulting orientation state. The modeling effort employed a so-called 'polydomain' description of orientation which facilitates direct connection to experimental measurements of orientation. This effort is greatly facilitated by a close analogy with fiber orientation models used in composite process modeling. The modifications that were made to the Moldflow software are an intellectual product that will be of benefit to the field when predictions to optimize injection molding processing is desired.

The project activity contributed to the progress of one graduate student (Mr. Jun Fang) at Northwestern University towards gaining his doctorate and to the progress of two undergraduate students at Saginaw Valley State University towards gaining their B.S. degrees.

For a more detailed summary, please see the PDF file attached to this final report.

\section{Findings:}

Key Findings:

The influences of molding parameters and mold thickness on the anisotropy and 'skin-core' morphology of injection-molded plaques fabricated from two distinct thermotropic liquid crystalline copolyesters (TLCPs). These are: (1) a 4'-dihydroxy-alpha-methylstilbene (DHaMS) containing copolyester , and (2) commercially available Ticona VectraÖ A950 6-hydroxy-2-naphthoic acid/6-hydroxybenzoic acid (HBA/HNA) copolyester. The contributions of shear and extensional flows on the bimodal orientation character of the moldings were characterized by a combination of 2-D WAXS in transmission, near-edge X-ray absorption fine structure (NEXAFS), and Attenuated Total Reflection Fourier Transform Infrared (FTIR-ATR) dichroism. Key observations and milestones include:

The influences of molding parameters and mold thickness on the anisotropy, skin-core morphology, and mechanical properties of injection molded DHaMS copolyester and Vectra thermotropic liquid crystalline copolyesters were studied. Key observations and milestones for the project include:

1. An extensive NEXAFS surface survey was accomplished for an injection molded plaque. Up to now, a limited number of areas off-axis from the centerline of plaques has been interrogated by using small squares of material cut from the plaques of interest. The new 6-axis manipulator 
at the U7A end station enabled for the first time the broad mapping of surface orientation of 2-dimensional net-shaped parts (beyond simple tensile bars) in the form of $76 \mathrm{~mm} \times 51 \mathrm{~mm}$ narrow-gated plaques. The narrow-gated plaques are of greater interest than the coat hanger gated plaques (which were also studied) because of their flow complexity.

2. A Herrick Seagull« Variable Angle Reflectance Attenuated Total Reflection Fourier Transform Infrared (FTIR-ATR) dichroism accessory was used as a complementary technique in the determination of surface orientations. The cross correlation and agreement of the surface orientation (magnitude and direction) results with NEXAFS measurements was quite good.

3. The physical properties of commercially available Vectra « A950 are influenced qualitatively by processing parameters in a fashion similar to that for the DHaMS copolyester; however, there are differences in their relative magnitudes and dependencies on anisotropy. Complementary 2-D wide-angle X-ray scattering measurements and the determination of the anisotropy factors (AF) that correspond to the measured properties were completed.

4. Larson-Doi simulations within the Moldflow« simulation platform successfully predict many details in the surface/skin (as outlined in this final report) and bulk orientation distributions induced during injection molding of commercial liquid crystalline polymers. This success is remarkable given the range of assumptions and approximations implicit in these calculations, such as decoupling of fluid mechanics and microstructural evolution, setting defect density equal to the local instantaneous shear rate, and so on.

5. The combination of NEXAFS, FTIR-ATR, and 2-D WAXS data provided an effective template to verify the success of the modeling efforts. The simulation results also demonstrate the effectiveness of the fiber model analogy, which has permitted the performance of simulations using existing and commercially available software, Moldflow «.

\section{Training and Development:}

The project under grant DMI-0521771 currently had the following educational and outreach aspects:

1. Jun Fang, who is a graduate student working directly with Prof. Burghardt at Northwestern University, worked with me under my direction in synchrotron-based soft X-ray spectroscopy performed key at the National Synchrotron Light Source at Brookhaven National Labs. He has written a new macro to automatically translate and rotate azimuthal and incident angles while maintaining fixed incident beam positions with the new 6-axis installed in the experimental station at U7A. He is due to receive his Ph.D. in early 2010.

2. Heather Fondriest, who is undergraduate mechanical engineering major at Saginaw Valley State University reporting directly to Mr. Bruce Hart, is completed obtaining new mechanical data on injection-molded Vectra plaques in collaboration with me. As opposed to the proprietary work that passes through testing facility at SVSU, the work in this academic grant project provides a research activity that can be openly discussed, as well as be a source of some modest financial support. Ms. Fondriest's curriculum did include aspects of automotive engineering and the materials component of this USCAR-supported (United States Council for Automotive Research) work has relevance to their course work. Ms. Fonderist received her B.S. degree in June 2008 and is now employed by Chrysler.

3. Susan M. Burgard is a technical cooperative education student employed with Impact Analytical, the characterization arm of MMI. She is an undergraduate biology major with a minor in chemistry at SVSU and collaborated in a cooperative project directly related to this grant under the direction of myself and Ms. Katherine Robertson (Impact analytical) performing Attenuated Total Reflectance û Fourier Transform Infrared (ATR-FTIR) spectroscopy measurements. The cooperative project is defined to characterize a specific sample set with samples in common with the mechanical and synchrotron-based measurements being made under the grant. Ms. Burgard received her BS in Biology from SVSU in June, 2009 and is currently employed by Impact Analytical.

4. I am a member of Mr. Jun Fang's doctoral committee at Northwestern University. Mr. Fang was principally supported in his PhD research in large measure under NSF Grant DMI-0521823, which is Prof. Burghardt's portion of our Co-PI grant that funds work on processing thermotropic liquid crystalline polymers. My participation on the committee was supported by NSF Grant DMI-0521771.

\section{Outreach Activities:}

\section{Journal Publications}

R. A. Bubeck, S. Rendon, W. R. Burghart, and L. S. Thomas, "Mechanical anisotropy in thermotropic liquid crystalline copolymer injection-moldings", 13th International Conference on Deformation, Yield, and Fracture of Polymers, p. 327, vol. 13, (2006). Published, 
R. A. Bubeck, L. S. Thomas, S. Rendon, W. R. Burghardt, A. Hexemer, D. A. Fischer, "Characterization of the skin orientation of thermotropic liquid- crystalline copolyester moldings with near-edge X-ray absorption fine structure", Journal of Applied Polymer Science, p. 2473, vol. 98, (2005). Published,

S. Rendon, W. R. Burghardt, R. A. Bubeck, L. S. Thomas, B. Hart, "Mechanical and morphological anisotropy in injection molding of thermotropic liquid crystalline copolyesters", Polymer, p. 10202, vol. 46, (2005). Published,

S. Rendon, R. A. Bubeck, L. S. Thomas, W. R. Burghardt, A. Hexemer, and D. A. Fischer, "Interrogation of 'surface', 'skin', and 'core' orientation in thermotropic liquid-crystalline copolyester moldings by near-edge X-ray absorption fine structure and wide-angle X-ray scattering", Journal of Applied Polymer Science, p. , vol. 106, (2007). Published, 10.1002

S. Rendon, W. R. Burghardt, and R. A. Bubeck, "Orientation dynamics in commercial thermotropic liquid crystalline polymers in transient shear flows", Rheologica Acta, p. , vol. 46, (2007). Published, 10.1007/s00397-007-0177-8

J. Fang, W. R. Burghardt, and R. A. Bubeck, "Polydomain Model Predictions of Molecular Orientation in Isothermal Channel Flows of Thermotropic Liquid Crystalline Polymers", Polymer Engineering and Science, p. 2258, vol. 48, (2008). Published,

Rendon, S.; Fang, J.; Burghardt, W. R.; Bubeck, R. A., "An Apparatus for in situ X-ray Scattering Experiments during Polymer Injection Molding", Review of Scientific Instruments, p., vol. 80, (2009). Accepted,

Fang, J.; Burghardt, W. R.; Bubeck, R. A.; Burgard, S. M.; Fischer, D. A., "Bulk and Surface Molecular orinetation Distribution in Injection Molded Liquid Crystalline Polymers: Experiment and Simulation", Polymer Engineering and Science, p. , vol. , (2009). Submitted,

Fang, J.; Burghardt, W. R.; Bubeck, R. A., "'In situ X-ray Scattering Measurements and Polydomain Simulations of Molecular Orientation Development during Injection of Liquid Crystalline Polymers", Proceedings of the XVth International Congress on Rheology, p. 39, vol. 1027, (2008). Published,

\section{Books or Other One-time Publications}

R. A. Bubeck, L. S. Thomas, K. Robertson, and S. L. Berets, "Characterization of "Skin" Orientation of Thermotropic Liquid Crystalline Copolyester Moldings", (2006). Technical note, Published

Editor(s): Not applicable.

Collection: Not applicable.

Bibliography: Herrick Scientific Products Technical Note No. 61101

R. A. Bubeck, J. Fang, W. R. Burghardt, S. Burgard, K. Robertson, D. Fischer, "Surface Orientation in Injection-Molded Thermotropic Liquid Crystalline Copolyester (TLCP) Plaques", (2008). Abstract, Published

Editor(s): Not applicable

Collection: APS March Meeting, New Orleans, LA

Bibliography: Paper R1 17

R. A. Bubeck, J. Fang, W. R. Burghardt, "In situ X-ray Scattering Measurements and Polydomain Simulations of Molecular Orientation Development during Injection Molding of Liquid Crystalline Polymers", (2009). Abstract, Published

Editor(s): Not applicable

Collection: APS March Meeting, Pittsburgh, PA

Bibliography: Paper L20.07

R. A. Bubeck, J. Fang, W. R. Burghardt, "Orientation Distribution and process Modeling of Thermotropic Liquid Crystalline Copolyester (TLCP) Injection Moldings", (2009). Abstract, Published

Editor(s): Not applicable

Collection: APS March Meeting, Pittsburgh, PA

Bibliography: Paper L20.07 
J. Fang, W. R. Burghardt, R. A. Bubeck, "Polydomain Simulation of Liquid Crystalline Polymer Orientation in Isothermal Extrusion Channel Flows", (2008). Abstract, Published

Editor(s): Not Applicable

Collection: APS March Meeting, New Orleans, LA

Bibliography: Paper C1 17

\section{Web/Internet Site}

\section{Other Specific Products}

\section{Contributions}

\section{Contributions within Discipline:}

The new results on orientation as a function of processing conditions were incorporated into modifications of modeling programs (e.g. Moldflow «). This activity is enhancing the capabilities to optimize the processing of thermotropic liquid crystalline polymers (TLCPs) in the fabrication of strong light weight net-shape parts for transportation applications, among others. As an additional benefit, the close corrolation of orientation determinations from the two surface spectroscopy techniques, NEXAFS and FTIR-ATR, was demonstrated experimentally for the first time.

The results will have an impact upon the goals of the United States Council for Automotive Research (USCAR) and the transportation industry, in general, to improve efficiencies and lower energy consumption. We have also been in communication with L\&L Products, Romeo, MI. They are suppliers to the automotive and other industries and continue to have an interest in exploiting improved processing technologies such as the route being explored under our current grant to take advantage of the properties of TLCPs in new engineering applications.

\section{Contributions to Other Disciplines:}

\section{Contributions to Human Resource Development:}

The project under grant DMI-0521771 currently had the following educational and outreach aspects:

1. Jun Fang, who is a graduate student working directly with Prof. Burghardt at Northwestern University, worked under my direction in synchrotron-based soft X-ray spectroscopy and performed key at the National Synchrotron Light Source at Brookhaven National Labs. Mr. Fang will be receiving his Ph.D. in early 2010.

2. Heather Fondriest, who was undergraduate mechanical engineering major at Saginaw Valley State University reporting directly to Mr. Bruce Hart, helped obtain mechanical property data on injection-molded Vectra plaques in collaboration with me. As opposed to the proprietary work that passes through testing facility at SVSU, the work in this academic grant project provides a research activity that can be openly discussed, as well as be a source of some modest financial support. Ms. Fonderist received her B.S. degree in June, 2008 and is now employed by Chrysler.

3. Susan M. Burgard was a technical cooperative education student employed with Impact Analytical, the characterization arm of MMI. She did a cooperative project directly related to this grant under the direction of myself and Ms. Katherine Robertson (of Impact Analytical) performing Attenuated Total Reflectance - Fourier Transform Infrared (ATR-FTIR) spectroscopy measurements. Ms. Burgard graduated in June 2009 from SVSU with a B. S. in Biology. She is now employed by Impact Analytical.

\section{Contributions to Resources for Research and Education:}

\section{Contributions Beyond Science and Engineering:}

\section{Conference Proceedings}




\section{Categories for which nothing is reported:}

Activities and Findings: Any Outreach Activities

Any Web/Internet Site

Any Product

Contributions: To Any Other Disciplines

Contributions: To Any Resources for Research and Education

Contributions: To Any Beyond Science and Engineering

Any Conference 


\title{
Principal Investigator/Project Director: Dr. Robert A. Bubeck
}

\author{
Institution: Michigan Molecular Institute
}

Award Number: DMI-0521771

Program: MPM

Project Title: Microstructural Modeling and Synchrotron Studies of Orientation
Development in Injection Molding of Liquid Crystalline Polymers

\author{
Comparative Determinations of Surface Orientation in Injection-Molded Thermotropic \\ Liquid Crystalline Copolyester (TLCP) Plaques - Final Report August, 2009
}

\author{
Robert A. Bubeck, Senior Research Scientist and Professor \\ Michigan Molecular Institute, Midland, MI 48640 \\ Wesley R. Burghardt (co-PI), Professor and Chair \\ Department of Chemical and Biological Engineering \\ Northwestern University \\ Evanston, IL 60208
}

\begin{abstract}
Melt processing thermotropic liquid-crystalline polymers (TLCPs) usually results in the fabrication of molded articles that possess complex states of orientation that vary greatly as a function of thickness and part geometry. This condition gives rise to "skin-core" morphologies and sample anisotropy. In order to better understand how processing conditions influence anisotropy, samples were fabricated from two different commercial thermotropic liquid crystalline copolyesters. Complementary mechanical property data for more than one TLCP for the sake of comparison were obtained. Attenuated Total Reflectance Fourier Transform Infrared (FTIR-ATR) dichroism and Near-edge Xray Absorption Fine Structure (NEXAFS) were utilized in conjunction as two means of determining surface layer orientation. The NEXAFS data were analyzed to determine the state of molecular orientation of the nematic polymers on the very near surface (depth of $\sim 2 \mathrm{~nm}$ ). These results were used in combination with FTIR-ATR dichroism (depth of $\sim 5 \mu \mathrm{m}$ ) and 2-D wide-angle X-ray scattering (2-D WAXS, depth of $\sim 0.5$ micron) in transmission to characterize to various probe depths the orientation in the "skin" and "core" regions of the injection-molded plaques. The results are the first for obtaining a complete surface orientation survey of a true two-dimensional net-shaped injection-molded part (beyond a simple tensile bar) and a successful complementary verification of the two spectroscopic techniques. These results were compared with the modeling of the processing of TLCPs with 'polydomain' simulations based upon Larson-Doi and Folger-Tucker models within the framework of the Moldflow ${ }^{\circledR}$ software package. Agreement between the simulation and experiments was found to be very good.
\end{abstract}




\section{INTRODUCTION}

Thermotropic liquid crystalline polymers (TLCPs) combine the virtues of superior tensile properties with the ability to injection mold with very easy flow through the spontaneous ordering of molecules. The critical processing issue is the development of high anisotropy during TLCP processing. Although the concurrence of high tensile properties and high directional orientation is of great benefit in fiber spinning, severe anisotropy can be a plague in obtaining balanced properties in net-shape injection-molded parts. The rigid nature of the mesogenic segments in TLCP molecules usually leads directly to a high orientational bias favoring the direction of flow with injection molding and other directional processing of thermotropes. ${ }^{1,2}$ Upon recrystallization, the high molecular orientation often leads to very favorable properties in the direction of orientation and lower physical properties in the transverse direction.

The high specific strength of TLCPs makes them excellent potential candidates for the manu-facture of strong lightweight net-shape parts intended for use in transportation applications. The singular challenge in affecting their use in this manner is gaining control over the severe anisotropy that can often result during melt processing. Towards this end, our research was targeted at: (1) improved understanding of how processing conditions influence orientation and physical properties; and (2) using the results to develop new modeling strategies for injection-molding to predict orientation formation based upon a socalled 'polydomain' description of orientation which facilitates a direct connection to experimental measurements of orientation. An important part of this research effort is an extensive 3-D mapping of orientation in fabricated parts processed under defined conditions with model TLCPs. A final summary of the progress in this effort is presented in this final report.

Characterizations were previously performed for the direction of greatest strength in relatively narrow injection-molded tensile bars fabricated from Celanese Vectra ${ }^{\circledR}$ 6-hydroxy-2-naphthoic acid/6hydroxybenzoic acid (HBA/HNA) type copolyesters by Dreher, et al. ${ }^{3}$ These researchers showed that tensile modulus increases linearly with the Hermans orientation function. Molding a plaque or other part with a broad aspect ratio, however, will incur much greater flow complexity and, therefore, more complex states of orientation. Shear flow dominates near the surface while transverse stretching dominates near the mid-plane ${ }^{4}$ resulting in bimodal cross-ply orientation, as schematically shown in Figure 1. Depending upon thickness, a "skin/core" structure also results with a high molecular alignment in the 'skin' that may be maximized in a direction different from that in the "core". Shear asserts increasing dominance with decreasing sample thickness. Accordingly, the emphasis in this study concerns the molding of plaques under a systematic set of conditions and thicknesses, and then characterizing the resulting bimodal orientations.

Near-edge X-ray absorption fine structure (NEXAFS) was used to determine the molecular orientation in the near surface ( $2 \mathrm{~nm}$ deep) across injection molded plaques. A novel 6-axis sample manipulator was utilized to facilitate the physical translation, and the incident and azimuthal angle rotations of the sample in the experimental chamber of U7A. The NEXAFS results were compared with surface orientation determinations made by using Attenuated Total Reflectance Fourier Transform Infrared (FTIR-ATR) dichroism. The results from NEXAFS and FTIR-ATR were then comprehensively compared to define the state of surface orientation in the plaques. These results along with other orientation determinations made by 2-D wide-angle X-ray scattering (2D-WAXS) were used to guide the development of improved modeling of the processing of TLCPs within the framework of the Moldflow ${ }^{\circledR}$ software package. 


\section{EXPERIMENTAL}

\section{2. a. Materials}

Three thermotropic liquid crystalline polymers were included in the study. For the final year's work, Ticona Vectra ${ }^{\circledR}$ A950 was being emphasized, which is a random copolyester consisting of $73 \%$ hydroxybenzoic acid (HBA) and 27\% 2,6-hydroxynaphthoic acid (HNA). The Vectra ${ }^{\circledR}$ A950 with a molecular weight in the environs of about $35,000 \mathrm{~g} / \mathrm{mol}$ was supplied by Ticona.

\section{2. b. Plaque Fabrication}

Sample plaques were fabricated using a Boy 30T2 injection molding machine with which molding parameters were controlled. Two plaque configurations are being studied. Type I plaques intended for micro-tensile testing measure $76.2 \mathrm{~mm} \times 76.2 \mathrm{~mm}$ and are molded with a coat hanger gate. Type II plaques intended for flow analysis are $76.2 \mathrm{~mm} \times 50.7 \mathrm{~mm}$ with a narrow gate located at the midpoint of one of the $76.2 \mathrm{~mm}$ sides. The molds were made by Master Precision Mold Technology, Inc. (Greenville, MI) and were fitted with an insert with a polished face that permitted the fabrication of plaques of various thicknesses. These were selected to be $0.8 \mathrm{~mm}, 1.6 \mathrm{~mm}$, and $3.2 \mathrm{~mm}$. Typically, the total cycle time was $47 \mathrm{sec}$, the screw speed was $264 \mathrm{rpm}$, pack (holding) pressure was 280 bar $\left(2.8 \times 10^{7} \mathrm{~Pa}\right)$, and the cooling (hold) time was $16 \mathrm{sec}$. Injection fill times of $1 \mathrm{sec}$ and $5 \mathrm{sec}$ were studied and these two times were obtained by varying injection pressure alone to the two values of 1391 bar $\left(1.39 \times 10^{8} \mathrm{~Pa}\right)$ and 140 bar $\left(1.40 \times 10^{7} \mathrm{~Pa}\right)$, respectively. In addition to a range of melt temperatures, two mold temperatures $\left(90^{\circ} \mathrm{C}\right.$ and $45^{\circ} \mathrm{C}$ ) were studied.

It was ascertained that the samples required surface cleaning to remove contaminants before the NEXAFS and FTIR-ATR dichroism examination. Cleaning was performed with a Bransonic 220 ultrasonic cleaner for duration of $5 \mathrm{~min}$. using a $1 \%$ solution of Alconox Liqui-Nox ${ }^{\circledR}$ cleaning agent in de-ionized water. Both the initial presence of contaminants and their effective removal were verified for selected samples by atomic force microscopy using a Topometrix 2000 AFM.

\section{2. c. Tensile Bar Fabrication and Testing}

Micro-tensile bars were machined from the plaques in directions both parallel and perpendicular to the nominal flow direction using a computer programmed end mill. The milling machine was programmed to cut three identical tensile samples parallel to or four samples perpendicular to the principal flow direction, as outlined in previous NSF annual reports. Thus, the middle sample parallel to flow was perfectly aligned to the direction for principal flow and, therefore, maximum orientation. The micro-tensile bars were tested at a deformation rate of $1.27 \mathrm{~mm} / \mathrm{min}$ using an Instron Model 1125 test frame with MTS ReNew@ Upgrade Package equipped with an MTS Model 632.29E30 Option 012 extensometer. The standard ASTM D-638 "Standard Test Method for Tensile Properties of Plastics" 5 was used as a guide, but tensile testing procedures were adapted to the specimen dimensions adopted for this study. An average of a minimum of five replicates was reported as the final value.

\section{2. d. Near-Edge X-ray Absorption Fine Structure (NEXAFS) for Surface Orientation}

The contribution to the orientation of the skin layer is difficult to deconvolute from that of the core of a molding. Dreher and coworkers ${ }^{3}$ determined skin orientation in TLCP moldings using WAXS of microtomed layers. A less laborious and less intrusive means of determining surface orientation presents itself in the form of near edge X-ray absorption fine structure (NEXAFS). NEXAFS is a synchrotron 
source UV spectroscopy technique that is sensitive to the orientation of phenyl groups in the direction of melt flow via the intensity $(I)$ of the partial electron yield $(P E Y)$ of Auger electrons of $1 \mathrm{~s} \rightarrow p i *$ transition of the $\mathrm{C}=\mathrm{C}$ bonds in the $\mathrm{C}$ K edge. The technique using $P E Y$ is sensitive to the top $2 \mathrm{~nm}$ of a surface. The orientation is determined through a series of measurements of the $\mathrm{C} \mathrm{K}$ edge spectra, as shown in Figure 3 , over a range of incident angles, $\theta,\left(30^{\circ}, 40^{\circ}, 50^{\circ}, 60^{\circ}, 70^{\circ}, 80^{\circ}\right.$, and $\left.90^{\circ}\right)$ of a monochromated linearly polarized UV beam relative to the sample surface. This technique has been successfully used, as well, to determine the orientation of LC alignment on rubbed polyimide substrates by Stöhr and Samant ${ }^{6}$ and by Pattison et al. ${ }^{7}$

The NEXAFS was performed on the NIST/Dow Soft X-ray Materials Characterization Facility, U7A, at the National Synchrotron Light Source, Brookhaven National Labs. Using the technique of Stöhr and Samant, the $P E Y$ intensity $I(\theta)$ is predicted to take the form,

$$
I(\theta)=A+B \sin ^{2} \theta
$$

regardless of the degree of orientation. Based on the method by Kramer published by Pattison et al., ${ }^{8}$ the molecular orientation parameter, $S_{\text {Surface }}$, was calculated using:

$$
S_{\text {Surface }}=\left(1-\frac{2(A+B)}{A+\frac{B}{6 P}(3 P-1)}\right)
$$

where, $P$ is the beam polarization ( 0.85 in this case), and $A$ is the intercept and $B$ is the slope of the linear plot of intensity vs. $\sin ^{2}(\theta)$ in Figure 3. A discussion of the application of NEXAFS to the measurement of surface orientation in TLCPs and the presentation of an extensive range of results is covered by Rendon et al. ${ }^{8}$

In our experimental setup, a new and novel 6-axis sample manipulator was utilized to facilitate the translation and the incident and azimuthal angle rotations of sample. Plaque samples were cut in half at their centerlines to accommodate by size the space available at the maximum translational and rotational displacements in using the 6-axis manipulator installed in the experimental chamber of U7A. Symmetry of flow was assumed. Each of nine positions of a plaque sample, as indicated in Figure 6, was interrogated by performing NEXAFS for the displacements and the incident and azimuthal angles required for the determination of the maximum $S$ and its respective azimuthal angle at each position. This approach totally eliminates the laborious manual rotation of small sample squares cut from plaques at positions of interest, as previously done in order to elucidate the molecular orientation at off-centerline locations. ${ }^{3}$ A correction scheme in the form of a macro to control the manipulator driver to offset the displacement of locations caused by the azimuthal and vertical angular rotations of sample plaque was written and used. It enables the soft x-ray beam to closely hit the exact same spot each time the sample was rotated azimuthally and the incident angle was adjusted.

\section{2.e. Attenuated Total Reflectance Fourier Transform Infrared (FTIR-ATR) Dichroism}

Layer orientation in injection molded tensile bars of 6-hydroxy-2-naphthoic acid/6-hydroxybenzoic acid (HBA/HNA) random type copolyesters was determined by Pirnia and Sung ${ }^{9}$ using Attenuated Total Reflection Fourier Transform Infrared (FTIR-ATR) dichroism. This technique enabled these researchers to determine relative orientation encompassing a depth of material 5 microns below the sample surface. Using measured dichotic ratios to calculate the orientation parameter, the skin, intermediate layers and 
core were characterized for a series of samples cut from positions along injection-molded plaques by progressively removing material by milling. A study of the surface orientation of injection-molded Vectra ${ }^{\circledR}$ B900 samples by a similar means was reported by Besaad, Jasse, and Noël. ${ }^{10}$

For the work presented here, the sample surface orientations were analyzed by infrared spectroscopy using the Seagull® Variable Angle Reflectance Accessory in a Thermo-Nicolet Nexus 670 FTIR spectrometer. The Seagull was equipped with a Ming-Sung ATR Rotator, a ZnSe ATR crystal, and a wire grid polarizer. The polarizer was set for s-polarization and the Seagull accessory was set for an incident angle of $55^{\circ}$. This incident angle was selected to be slightly above the experimental critical angle for the samples examined. Each sample was oriented on the sampling stage of the ATR Rotator so its cut edges corresponded to the $0^{\circ}$ and $90^{\circ}$ degree settings. A background spectrum was recorded using the clean ATR crystal without a mounted sample and then sample spectra were collected with the ATR Rotator set at $-10^{\circ}$ to $90^{\circ}$ in $10^{\circ}$ increments. The spectra collected were signal averaged over 32 scans with $4 \mathrm{~cm}^{-1}$ resolution, over a spectral wavenumber range from $4000 \mathrm{~cm}^{-1}$ to $650 \mathrm{~cm}^{-1}$. Data were normalized using the OMNIC Software, v.6.1A provided by Nicolet. Considerable effort was expended to resolve any instrumental issues and assure the reproducibility of the data. Having an inventory of $0.8 \mathrm{~mm}$ thick samples previously characterized using NEXAFS and 2-D WAXS was of great benefit in accomplishing this task. It was found that: (1) the use of a calibrated torque wrench to tighten the sample back plate of the Seagull ZnSe ATR crystal stage to obtain optimum crystal-sample contact, and (2) using samples sufficiently compliant molded (or milled on the back side) to a thickness of less than $1.6 \mathrm{~mm}$ are critical in obtaining good crystal to surface contact and good results.

Nine samples $\sim 1 \mathrm{~cm}$ by $1 \mathrm{~cm}$ square were cut from positions in the plaques corresponding to those studied using NEXAFS. The FTIR-ATR spectra were converted to absorbance units and baseline corrected. Two examples of IR spectra collected on an off-center location taken perpendicular [A] and parallel [B] to the preferential orientation are shown in Figure 4 and the absorption band assignments are listed in a companion table with the assignment references ${ }^{9,17,18,19}$ cited. From these data, the dichroic ratios were determined using the $1504 \mathrm{~cm}^{-1}$ absorption band associated with in-plane aromatic ring bending. Orientation functions $\left(f_{\mathrm{xy}}\right)$ were calculated, as described by Pirnia and Sung ${ }^{4}$, from the acquired dichroic ratios $\left(D_{x y}=A_{x} / A_{y}\right)$ at predefined points in the plane of the surface, where $A_{x}$ and $A_{y}$ are spatial absorbances parallel and perpendicular to the principle molecular orientation, respectively. Orientation function $(f)$ is defined as,

$$
f=(D-1) /(D+2)
$$

The orientation function is comparable to the order parameter $\left(S_{\text {surface }}\right)$ derived from NEXAFS. Similarly, $f$ is equal to 1.0 for perfect flow direction orientation, whereas $f$ yields -0.5 for perfect transverse orientation. In case of an almost random configuration of the molecules, $f$ is close to zero. For each of nine interrogated position on the plaques, as indicated in Figure 6, the maximum for $f$ and its azimuthal angle was reported. As with NEXAFS, the azimuthal angle associated with maximal $f$ determines the local orientation angle associated with the near surface layer.

\section{2. f. 2-D WAXS Data and Analysis Procedures of Injection-Molded Plaques and the Determination of Anisotropy Factor (AF).}

An orientation map for each plaque type intended for tensile testing was determined by 2-D WAXS performed at $20 \mathrm{keV}(0.62 \AA)$ for multiple positions on the samples using the 5BM beam line of the DuPont/Northwestern University/Dow Collaborative Access Team at the Advanced Photon Source of Argonne National Lab. An incident X-ray beam with a $1 \mathrm{~mm}$ diameter size was used. A Mar CCD detector was used to collect $512 \times 512$ pixel raw X-ray scattering patterns with 15 -second exposures. The 
WAXS patterns were obtained in positions corresponding to a grid, points for which correspond to the critical positions for the tensile sections of the tensile samples machined from the plaques. There are significant bimodal contributions from both the 'core' and the 'skin' in the WAXS patterns obtained for the thicker plaques, an example of which is shown in Figure 2. Details of the data analysis techniques employed are given by Rendon, et al. ${ }^{11}$ The 2-D WAXS patterns usually indicate the presence of two discrete populations of orientation: one parallel to the flow direction and the other transverse to the flow direction. As evidenced in all of the patterns, nematic crystalline peaks always diffract perpendicular to the orientation direction. Scans made along this horizontal region of the plaque suggest the dominance of transverse orientation modes relative to shear-flow modes manifested by strong crystalline peaks, which are indicative of higher degrees of molecular orientation. Away from the centerline position, the bimodal character switches direction indicating that the transverse mode has rotated relative to molecular orientation at the centerline.

An assumption of uniaxial orientation is not appropriate when studying complex flow kinematics where both shear and extensional flows occur across the entire thickness of the sample. Instead, a measurement of anisotropy in the scattering pattern was obtained by computing the azimuthal intensity scan at $q^{*} .{ }^{12}$ This is done by taking a point on the azimuthal scan represented by the unit vector $\mathbf{u}$, such that $u_{1}=\cos \beta$ and $u_{2}=\sin \beta$, where $\beta$ is the characteristic azimuthal angle. Anisotropy can then be described by computing a weighted average of the second moment tensor of $\mathbf{u}$, via:

$$
\langle\mathbf{u u}\rangle=\left[\begin{array}{ll}
\left\langle u_{1} u_{1}\right\rangle & \left\langle u_{1} u_{2}\right\rangle \\
\left\langle u_{1} u_{2}\right\rangle & \left\langle u_{1} u_{2}\right\rangle
\end{array}\right]=\left[\begin{array}{ll}
\left\langle\cos ^{2} \beta\right\rangle & \langle\sin \beta \cos \beta\rangle \\
\langle\sin \beta \cos \beta\rangle & \left\langle\sin ^{2} \beta\right\rangle
\end{array}\right]
$$

In this case $\langle\cdots\rangle$ represents an average weighted by the azimuthal intensity distribution $I(\beta)$, where $\beta$ is measured from the flow direction, as shown in Figures 3 and 4. For instance, the average $\left\langle\cos ^{2} \beta\right\rangle$ is given by the expression:

$$
\left\langle\cos ^{2} \beta\right\rangle=\frac{\int_{0}^{2 \pi} \cos ^{2} \beta I(\beta) d \beta}{\int_{0}^{2 \pi} I(\beta) d \beta}
$$

An anisotropy factor $(A F)$ is then computed by taking the difference in the principal eigenvalues of the second moment tensor given as follows:

$$
A F=\lambda_{1}-\lambda_{2}=\sqrt{\left(\left\langle u_{1} u_{1}\right\rangle-\left\langle u_{2} u_{2}\right\rangle\right)^{2}+4\left\langle u_{1} u_{2}\right\rangle^{2}}
$$

In the limit of perfect orientation, independent of direction, this quantity will yield a value of 1 , while 0 reflects a randomized orientation state (i.e. isotropic). The average orientation direction is computed from the eigenvectors or principal directions of the second moment tensor.

Anisotropy measurements made along the centerline region of the plaque will simply amount to taking the difference in the diagonal components of equation $4,\left\langle u_{2} u_{2}\right\rangle-\left\langle u_{1} u_{1}\right\rangle=\left\langle\sin ^{2} \beta\right\rangle-\left\langle\cos ^{2} \beta\right\rangle$. Symmetry dictates that the off-diagonal components of the tensor be zero. For this investigation, a coordinate system was adopted in which the 1-axis is oriented along the prevailing shear-flow direction. Consequently, 
values of the Anisotropy Factor $(A F)$ that approach +1 indicate perfect molecular orientation along the prevailing flow direction, while values approaching -1 correspond to perfect orientation transverse to the flow direction. It is assumed that the uniaxial orientation to compute the corresponding order parameters can be based upon the Herman's convention, viz,

$$
S=\frac{1}{2}\left(3 \cos ^{2} \beta-1\right)
$$

where in the limit of perfect orientation $S$ has a value of 1 , and 0 indicates a randomized orientation state. The procedures are described further by Bubeck and co-workers. ${ }^{9,13}$

\section{2. g. Simulations - A Brief Summary.}

The simulations developed here employ the Larson-Doi model ${ }^{22}$, which describes the orientation of textured 'polydomain' LCPs under flow. As in recent work evaluating Larson-Doi model predictions of TLCP orientation distributions in isothermal extrusion-fed channel flows, ${ }^{11}$ we drew upon an analogy with fiber orientation models to facilitate simulations of orientation during injection molding using commercial software tools. Complete details on this approach may be found in that study ${ }^{11}$ and in a more recently submitted paper by Fang et al. ${ }^{23} \mathrm{~A}$ few descriptive comments addressing the modeling approach are to be found in the discussion that follows.

The simplest theory to predict TLCP dynamics under flow is the Ericksen model ${ }^{25}$, written as an evolution equation for the nematic director, $\mathbf{n}$, which indicates the direction about which molecules are spontaneously ordered in the liquid crystalline state:

$$
\frac{\partial \mathbf{n}}{\partial t}=\mathbf{n} \cdot \omega+\lambda(\mathbf{n} \cdot \mathbf{D}-\mathbf{n n n}: \mathbf{D}) .
$$

Here $\mathbf{D}$ and $\boldsymbol{\omega}$ are, respectively, the rate of deformation and vorticity tensors, and $\lambda$ is a material constant known as the tumbling parameter. Direct measurements of $\lambda$ have not been possible in commercial TLCPs. However, the molecular orientation behavior observed in simple shear and complex channel flows suggests that they are of the tumbling type ${ }^{11,25}$, corresponding to $|\lambda|<1$. In this case, Eqn. (8) is identical to the equation governing the orientation of an isolated, axisymmetric particle suspended in a viscous liquid under flow (in which case $\lambda$ is determined by the particle's aspect ratio). This analogy is central to our modeling strategy.

Because the fundamental hydrodynamics of the nematic director and elongated particles are identical (Eqn. (8)), there is a nearly exact analogy between the Larson-Doi polydomain model for liquid crystal polymers and the Folgar-Tucker fiber orientation model ${ }^{18}$ widely used in composites processing. Since the Folgar-Tucker model is widely implemented in commercial polymer process simulators, this analogy facilitates direct application of existing software tools to perform the Larson-Doi simulations of interest.

The simulations were performed using Moldflow MPI ${ }$, a commercial software package that predicts flow fields during injection molding processing. This software incorporates the Folgar-Tucker fiber orientation model, allowing for corresponding microstructural predictions. Simulations were performed using a ' $2.5 \mathrm{D}$ ' mid-plane simulation method based on a Hele-Shaw approximation. ${ }^{26}$ For the plaque geometry considered here, this approach reduces computational effort while retaining a realistic description of the spatially varying mixed shear-extensional kinematics that are believed to be responsible for the orientation patterns generated during molding. ${ }^{11}$ 
The desired plaque geometry and associated mid-plane mesh were generated by tools within the MPI software suite. Process variables were chosen to match the real injection molding process, including filling time $(1 \mathrm{~s})$, cooling time $(16 \mathrm{~s})$, melt temperature $\left(290^{\circ} \mathrm{C}\right)$, and mold temperature $\left(45^{\circ} \mathrm{C}\right)$. In presenting and discussing computed orientation states, a Cartesian coordinate system was adopted in which ' $x$ ' denotes the filling direction and ' $z$ ' denotes the thin dimension of the plaque. In both surface and bulk orientation measurements, the molecular orientation state is probed in the $x-y$ plane. As a result, the following two-dimensional projection of the orientation tensor contains the relevant information for comparison to experimental data:

$$
\mathbf{T}=\left\lfloor\begin{array}{ll}
T_{x x} & T_{x y} \\
T_{x y} & T_{y y}
\end{array}\right\rfloor
$$

For the case of 2-D WAXS, the data reflect bulk orientation states averaged through the sample thickness, we report simulation results in terms of the thickness-averaged orientation tensor, $\langle\mathbf{T}\rangle$. At any desired location on the plaque, the difference in the principal values of $\langle\mathbf{T}\rangle$ provides a quantitative measure of the average degree of anisotropy:

$$
\text { Anisotropy factor }=\sqrt{\left(\left\langle T_{x x}\right\rangle-\left\langle T_{y y}\right\rangle\right)^{2}+4\left\langle T_{x y}\right\rangle^{2}},
$$

while the average direction of molecular orientation is obtained from the principal directions of $\langle\mathbf{T}\rangle$ within the $x-y$ plane. Defined by Equation 10, which is a restatement of Equation 6, the anisotropy factor ranges from 0 for a random isotropic distribution of orientation, to 1 for the perfect domain alignment in the $x-y$ plane. Along the centerline axis of a symmetric flow field, the principal directions will coincide with the $x$ and $y$ coordinate axes. This allows a simpler measure of anisotropy factor, $\left\langle T_{x x}\right\rangle-\left\langle T_{y y}\right\rangle$, which can range from +1 for perfect alignment along the $x$-direction, to -1 for perfect alignment along the transverse, $y$-direction. The similar definition of anisotropy factor derived from 2D-WAXS experiments facilitates direct quantitative comparison between experimental data and simulations. It is important to recognize that the polydomain simulations (and the associated second moment description of the orientation) only treat the distribution of director orientation. Conversely, experimental data also reflect the local spread of molecular orientation with respect to the director (as characterized by the molecular order parameter). As a result, it is anticipated that that anisotropy predicted by simulations will be systematically higher than corresponding experimental measures of molecular orientation. Predicted orientation distributions were extracted from the simulations at the same locations studied experimentally (Figure 7).

To obtain depth-resolved information on the molecular orientation state, the plaque thickness was discretized into 20 discrete laminae in the $z$-direction. Orientation distributions calculated in channel flow demonstrate that Larson-Doi simulations are capable of realistically predicting differences in orientation between a shear-dominated 'skin' and the 'core' where superimposed extension can dominate. ${ }^{24}$ Given the high surface specificity of the spectroscopies used here $(\sim 2 \mathrm{~nm}$ and $\sim 5 \mu \mathrm{m}$ for NEXAFS and FTIRATR, respectively), however, even the finest depth discretization possible within the software cannot provide direct predictions of the experimentally measured surface orientation states. Further, although the standard 'midplane' simulation approach used provides a realistic description of kinematics away from the filling front, it fails to predict three-dimensional phenomena like fountain flow, which are expected to strongly impact surface orientation. Previous NEXAFS and 2D-WAXS studies on a pre-commercial TLCP revealed, however, that the orientation in the aligned surface layer closely follows that of the 
thicker shear-dominated skin region. ${ }^{8}$ As a first test of ability of Larson-Doi simulations to capture aspects of the surface orientation distribution, we extracted predictions of skin orientation from the outermost simulation laminae, using procedures described in detail in reference 23.

\section{RESULTS AND DISCUSSION}

\section{3. a. Mechanical Properties and Their Correlation to Orientation Determined by}

\section{2-D WAXS.}

Tensile properties versus molecular orientation for $76 \mathrm{~mm}$ by $76 \mathrm{~mm}$ injection molded plaques is represented by tensile modulus and fracture stress versus $A F$ for the range of processing conditions and thicknesses studied and is plotted in Figure 5. The newly obtained data for the Vectra ${ }^{\circledR}$ A950 samples is superimposed on data for the DHaMS TLCP, which has been reported previously by Rendon et al. elsewhere. ${ }^{14}$ The samples with net negative values for $A F$ are those dominated by transverse extension during their molding while the samples with net positive values for $A F$ are those dominated by shear flow. The latter are populated principally by samples cut from the thinner plaques. The observed sharp increase in modulus with increasingly positive $A F$ is similar to and is consistent with what was indicated by data for the melt-drawing of HBA/HNA random copolyester TLCP filament by Garg and Kenig ${ }^{15}$, as plotted after the fact by Donald and Windle. ${ }^{16}$ Fracture stress was found to generally increase in a linear fashion with increasing $A F$ as well. The completed data obtained for Vectra ${ }^{\circledR}$ A950 plotted against $A F$ for the specific samples at hand indicates similar trends for tensile modulus, but elevated values vis-à-vis the DHaMS TLCP for fracture stress indicate that Vectra ${ }^{\circledR}$ A950 is the tougher of the two polymers.

\section{3. b. Examples of NEXAFS and FTIR-ATR Results for Surface Orientation on a Net- Shaped Injection-molded Part.}

NEXAFS and FTIR-ATR are two independent spectroscopic means of determining surface orientation. The FTIR-ATR dichroism measurements were undertaken to supplement the synchrotron-based NEXAFS measurements, and to yield additional information resulting from a technique that probes to a much greater depth from the surface ( 5 microns vs. $2 \mathrm{~nm}$ ) than the capability of C K edge PEY NEXAFS. The measurements by both techniques were taken from the identical plaque sample. The NEXAFS measurements were first obtained on the whole plaque, which was subsequently cut into nine $1 \mathrm{~cm} \times 1 \mathrm{~cm}$ squares corresponding to the positions interrogated by NEXAFS, as indicated in position key at the top of Figure 6.

The results for these two techniques for a $3.2 \mathrm{~mm}$ thick (originally) x $76 \mathrm{~mm}$ x $50.8 \mathrm{~mm}$ narrow-gated plaque injection-molded with Vectra ${ }^{\circledR} \mathrm{A} 950$ are shown in Figure 6 . The plaque was processed under the conditions of $290^{\circ} \mathrm{C}$ melt temperature and $45^{\circ} \mathrm{C}$ mold temperature. A comparison of Figures $6 \mathrm{a}$ (NEXAFS) and $6 \mathrm{~b}$ (FTIR-ATR) indicate that the agreement for $S$ and $f$ are very good and angles of maximum orientation are quite good for the most part. The results are the first for obtaining a complete surface orientation survey of a true two-dimensional injection-molded part (beyond a simple tensile bar) and a complementary verification of the two spectroscopic techniques. These results, along with the mechanical property determinations, were used to guide the development of the modeling of the processing of TLCPs with 'polydomain' simulations based upon Larson-Doi ${ }^{17}$ and Folger-Tucker ${ }^{18}$ models within the framework of the Moldflow ${ }^{\circledR}$ software package, as reported in ref. 23.

\section{3. c. Simulation Results for Surface Orientation Distribution.}

FTIR-ATR and NEXAFS were utilized to map out the surface orientation distribution at nine representative centerline and off-center locations (Figure 6). It is the first time that these two techniques, 
both sensitive to the orientation near to the top surface, are compared and further validated by each other. Numerical values of surface angle and orientation parameters are also listed for each location to facilitate quantitative comparisons. Clearly, the surface orientation distributions from NEXAFS (Figure 6a) and FTIR-ATR (Figure 6b) demonstrate good agreement with each other despite the different depths that are naturally probed using these two techniques. This is consistent with earlier observations that surface orientation closely tracks that found in the thicker skin layer encompassing both the immediate surface layer (examined here by NEXAFS and FTIR-ATR) as well as the thicker shear-dominated region. ${ }^{8,11}$ The measured surface order parameters are quite large (typically $\sim 0.7$ or greater), comparable to the highest degree of orientation found in bulk orientation experiments. It is believed that highly aligned surface layers are produced by 'fountain flow' and fast solidification during the mold filling. ${ }^{8}$ In accord with this concept, the surface orientation states at the farthest downstream location do not degrade, in contrast to the bulk orientation distribution where transverse extension generated as melt impinges on the end of the mold cavity leads to actual flipping of molecular orientation.

At locations close to the plaque edge (the three points farthest away from the centerline), it was noted that the surface orientation state does not align towards the prevailing filling $(x)$ direction observed in bulk orientation states. At these locations, polymer molecules at the surface have large azimuthal orientation angles relative to the centerline, ranging from $30^{\circ}$ to $60^{\circ}$. This evidence strongly supports the argument that the surface orientation states were set by rapid solidification experienced at the mold front during filling. Thus, the azimuthal orientation angles measured via FTIR-ATR and NEXAFS are set at the moment when polymer molecules were first injected into the mold cavity. Most notably, the orientation angle measured by FTIR-ATR and NEXAFS equals $30^{\circ}$ at the point farthest away from both the injection gate and the centerline, which decidedly differs from that (near $0^{\circ}$ ) found in bulk 2D-WAXS measurements. ${ }^{23}$ The directions of maximum orientation obtained by FTIR-ATR in the region of the lower right side of the plaque are often rotated away from the centerline clockwise by roughly $10^{\circ}$ compared to those obtained using NEXAFS (Figure 6). This is possibly due to an increased contribution from extensional flow at the greater depth below the surface to which FTIR-ATR probes $(\sim 5 \mu \mathrm{m})$.

\section{Surface vs. Bulk Orientation Distribution}

To better explore how orientation states evolve in either bulk or skin regions, we turned to the depthresolved orientation state data from the simulations. As discussed, the limited resolution of mesh elements generated by the simulation tool, MOLDFLOW ${ }^{\circledR}$, would not allow us to directly tackle the orientation states in the near surface regions, interrogated by NEXAFS $(\sim 2 \mathrm{~nm})$ and FTIR-ATR $(\sim 5 \mu \mathrm{m})$. Consequently, we extracted the orientation states attributed by skin region, approximately $0.4 \mathrm{~mm}(\sim 15 \%$ of plaque thickness) using procedures described in detail elsewhere. ${ }^{23,24}$ According to the previous work on DHaMS plaques with the same geometry, the magnitude of molecular orientation due to sheardominated skin region is lower than that due to near surface region but their orientation angles agree well with each other. ${ }^{8}$ In other words, understanding how orientation states evolve in skin region is a direct means to explore the distribution of their counterparts in near surface region.

Closest to the plaque edge ( $\sim 28 \mathrm{~mm}$ away from the centerline), bulk and surface/skin orientation states were found to differ strongly in both degree and direction of orientation. While the surface/skin orientation state is set at the point of rapid solidification of the materials adjacent to the cold mold cavity wall as the melt front first arrives, the bulk orientation state continues to evolve with further mold filling, receive a strong influence of the dominant shearing kinematics near to the edge bringing the orientation direction closer to the vertical filling direction. More extensive details of the characterization of orientation in the bulk are reported in ref. 23. 


\section{SUMMARY}

The influences of molding parameters and mold thickness on the anisotropy, skin-core morphology, and mechanical properties of injection molded DHaMS copolyester and Vectra thermotropic liquid crystalline copolyesters were studied. Key observations and milestones for the project include:

1. An extensive NEXAFS surface survey was accomplished for an injection molded plaque. Up to now, a limited number of areas off-axis from the centerline of plaques has been interrogated by using small squares of material cut from the plaques of interest. The new 6-axis manipulator at the U7A end station enabled for the first time the broad mapping of surface orientation of 2dimensional net-shaped parts (beyond simple tensile bars) in the form of $76 \mathrm{~mm}$ x $51 \mathrm{~mm}$ narrowgated plaques. The narrow-gated plaques are of greater interest than the coat hanger gated plaques because of their flow complexity.

2. A Herrick Seagull ${ }^{\circledR}$ Variable Angle Reflectance Attenuated Total Reflection Fourier Transform Infrared (FTIR-ATR) dichroism accessory was used as a complementary technique in the determination of surface orientations. The cross correlation and agreement of the surface orientation (magnitude and direction) results with NEXAFS measurements was quite good.

3. The physical properties of commercially available Vectra ${ }^{\circledR}$ A950 are influenced qualitatively by processing parameters in a fashion similar to that for the DHaMS copolyester; however, there are differences in their relative magnitudes and dependencies on anisotropy. Complementary 2-D wide-angle X-ray scattering measurements and the determination of the anisotropy factors $(A F)$ that correspond to the measured properties were completed.

4. Larson-Doi simulations within the Moldflow ${ }^{\circledR}$ simulation platform successfully predict many details in the surface/skin (as outlined in this final report) and bulk (as detailed in ref. 23) orientation distributions induced during injection molding of commercial liquid crystalline polymers. This success is remarkable given the range of assumptions and approximations implicit in these calculations, such as decoupling of fluid mechanics and microstructural evolution, setting defect density equal to the local instantaneous shear rate, and so on.

5. The combination of NEXAFS, FTIR-ATR, and 2-D WAXS data provided an effective template to verify the success of the modeling efforts. The simulation results also demonstrate the effectiveness of the fiber model analogy, which has permitted the performance of simulations using existing and commercially available software, Moldflow ${ }^{\circledR}$.

\section{ACKNOWLEDGEMENTS}

First of all, thanks to my principal collaborators in this work, W. Burghardt and J. Fang (Northwestern University). The collaborative efforts with S. Burgard, L. Thomas and K. Robertson (M.M.I.) are invaluable. The thoughtful and careful determination of physical properties by B. Hart and H. Fondriest was most helpful. Thanks are extended to D. Fischer and the staff of Beamline U7A of the National Synchrotron Light Source, Brookhaven National Labs. Funding support from the National Science Foundation and the Department of Energy through NSF Grant DMI-0521771 is gratefully acknowledged. The gift of $55 \mathrm{lbs}$. of Vectra ${ }^{\circledR}$ A950 to our effort by Ticona is greatly appreciated.

Vectra ${ }^{\circledR}$ is the trademark of Ticona, a business of Celanese, Inc.

Seagull ${ }^{\circledR}$ is a trademark of Herrick Scientific products, Inc.

Moldflow ${ }^{\circledR}$ is a trademark of Moldflow, Inc. 


\section{FIGURES}

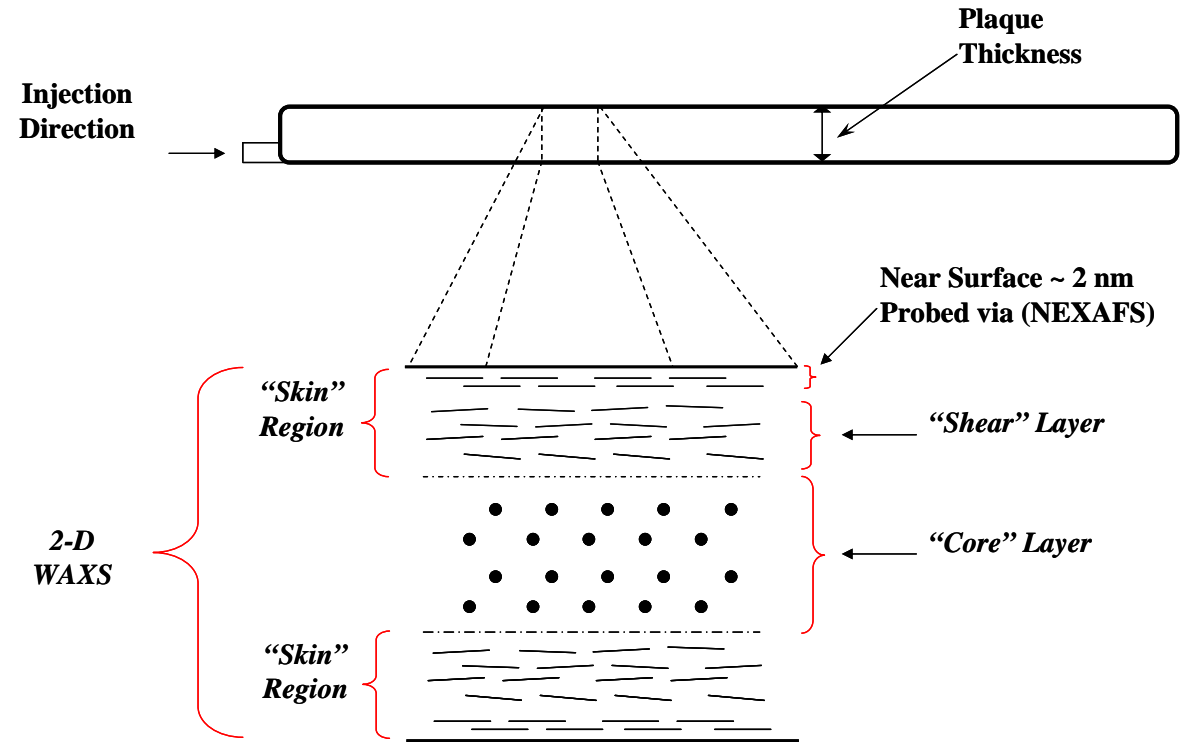

Figure 1. Idealized schematic of an injection molded plaque cross-section illustrating the levels of morphology present along the plaque thickness direction. 2-D WAXS in transmission averages structural information through the sample thickness while NEXAFS probes molecular orientation over depths of $2 \mathrm{~nm}$ in the near surface layer. FTIR-ATR probes to a depth of 5 microns. 


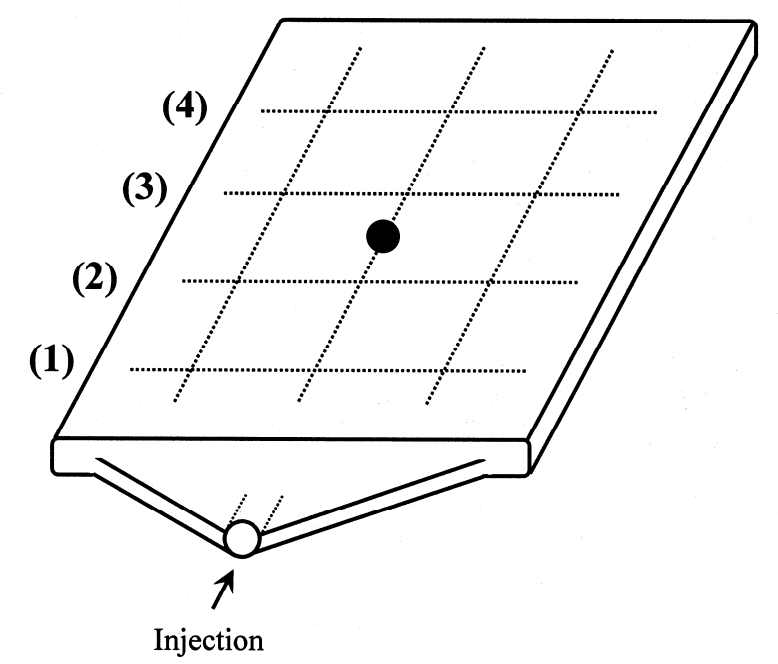

(a)

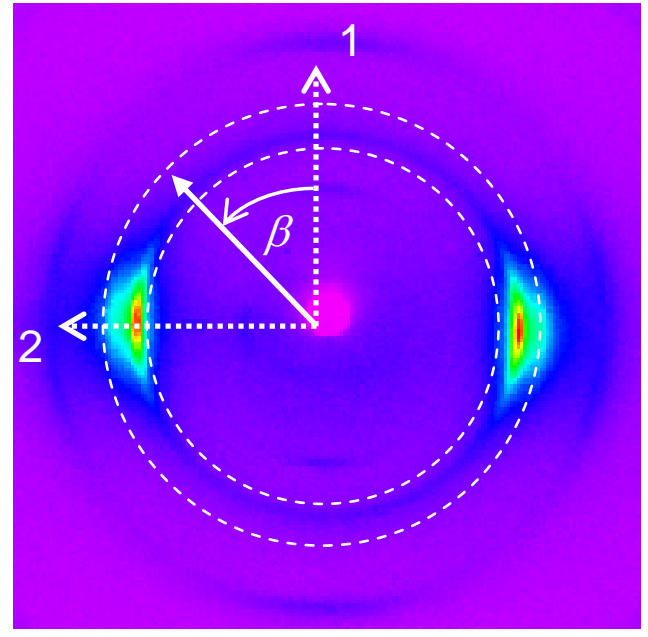

(b)

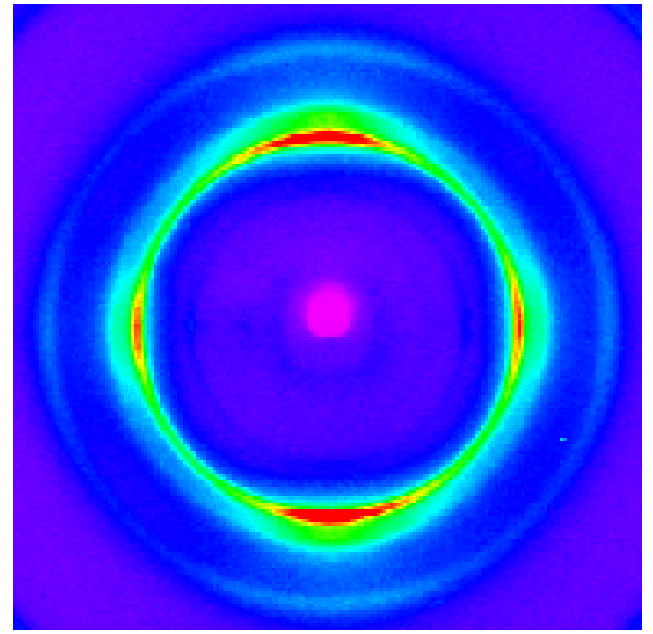

Figure 2. Examples of physical orientation of an injection-molded plaque along with two representative 2-D WAXS patterns taken for a centerline position of a: (a) $0.8 \mathrm{~mm}$ thick molded plaque showing uniaxial contribution to the orientation due to shear flow and a (b) $3.2 \mathrm{~mm}$ thick plaque showing bimodal orientation populations due to shear and extension. The arrow indicates the principal direction of flow. The plaques were processed at constant fast fill time of $1 \mathrm{~s}$ with melt and mold temperatures of $270^{\circ} \mathrm{C}$ and $45^{\circ} \mathrm{C}$, respectively. The overlays in (a) define the coordinates and range of the scattering wave vector, $q$, used for the extraction of azimuthal scans; $\beta$ is the azimuthal angle measured away from the vertical filling direction. Dotted lines indicate the path locations where 2-D WAXS measurements were performed $15 \mathrm{~mm}$ apart. 

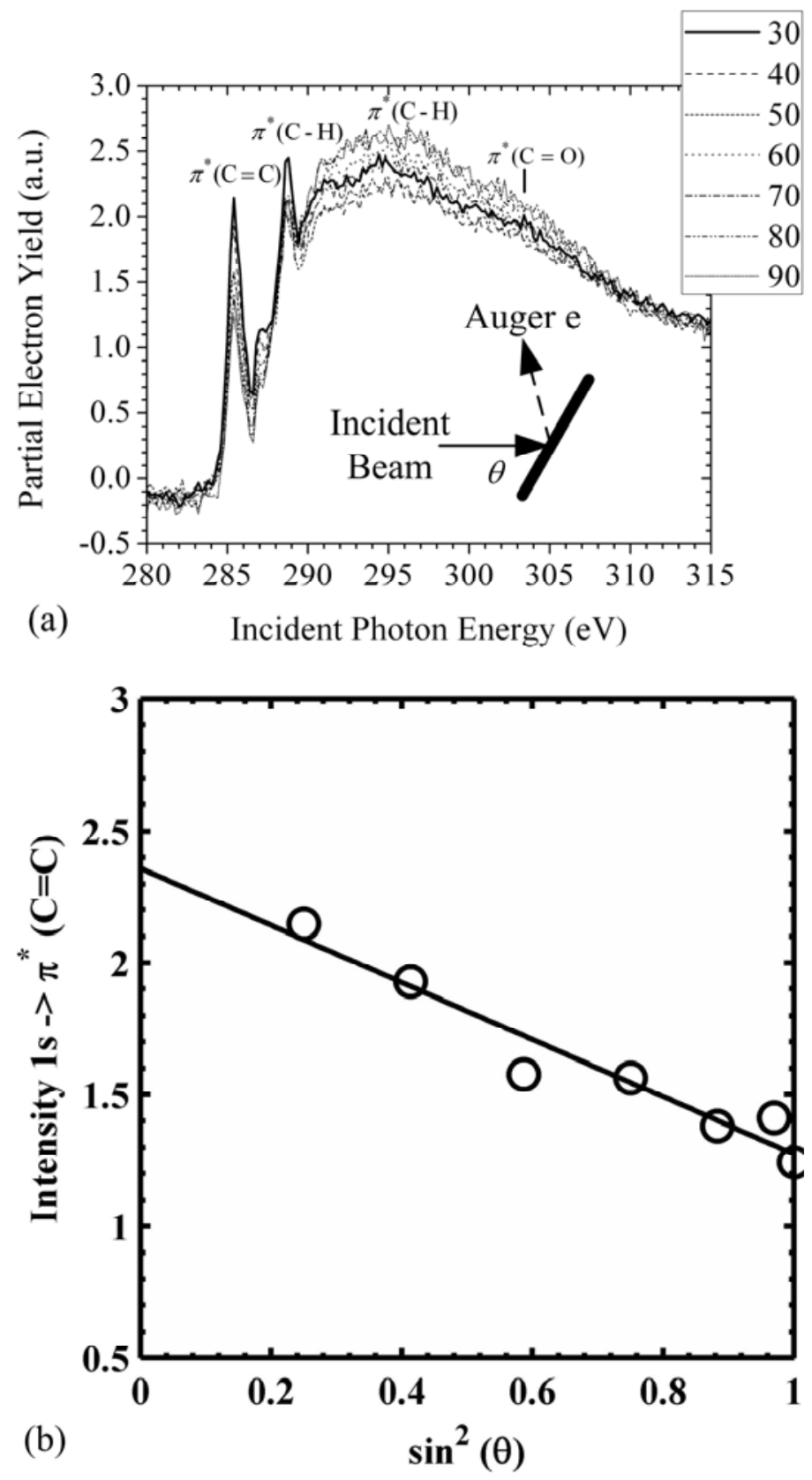

Figure 3. Example NEXAFS C K edge spectra of Vectra ${ }^{\circledR}$ A950 collected on a centerline location (denoted by $\bullet$ symbol in Fig. 2) and one essential data analysis procedure: a) plots of PEY (partial electron yield) versus incident photon energy at various incident angles $\left(30^{\circ}-90^{\circ}\right)$ and $0^{0}$ azimuthal rotation; the representative chemical groups associated with spectra peaks were also shown; b) the angular dependence of the phenyl ring $\mathrm{C}=\mathrm{C} \pi^{*}$ resonance parallel to the primary flow direction at the surface of this particular location as a function of the incident angle, $\theta$, where the intercept is A and the slope is B in Equation 1. 


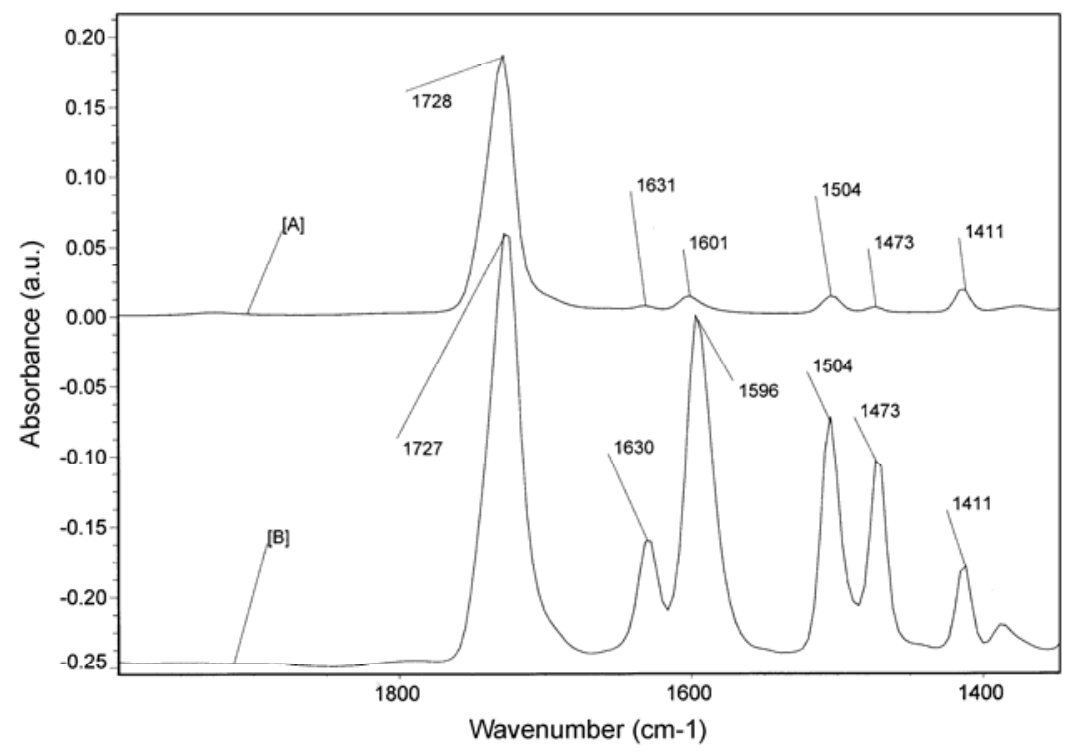

Absorption band wavenumbers in the range of interest for Vectra ${ }^{\circledR}$ A950.

\begin{tabular}{|c|l|}
\hline Wavenumber $\left(\mathbf{c m}^{-\mathbf{1}}\right)$ & \multicolumn{1}{|c|}{ Assignments (with Refs.) } \\
\hline 1411 & HBA skeletal vibration of phenyl ring $(9,19)$ \\
\hline 1473 & HNA skeletal vibration of naphthene $(9,19)$ \\
\hline 1504 & HBA phenyl in-plane ring bend $(9,20)$ \\
\hline 1600 & In-plane aromatic ring bend stretching $(2,19)$ \\
\hline 1630 & HNA skeletal vibration of naphthene $(9,20)$ \\
\hline 1727 & Carbonyl stretching (out of plane) $(9,21)$ \\
\hline
\end{tabular}

Chemical structure of Vectra ${ }^{\circledR}$ A950 (4-hydroxybenzoic acid (HBA) - 6-hydroxy-2-naphthoic acid (HNA))

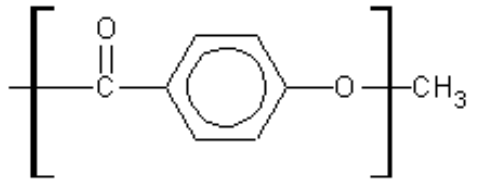

0.73

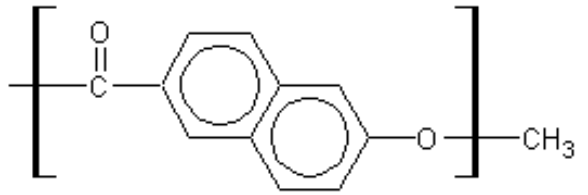

0.27

Figure 4. Two example normalized ATR-FTIR spectra collected at an off-center location. Spectra [A] and [B] were taken, respectively, for the directions perpendicular and parallel to the direction of maximum orientations. The absorption band assignments for Vectra ${ }^{\circledR}$ A950 are listed in the companion table. Dichroic ratios (D) for the determination of orientation functions $(f)$ were obtained using the 1504 $\mathrm{cm}^{-1}$ absorption band, as per equation 2 . 

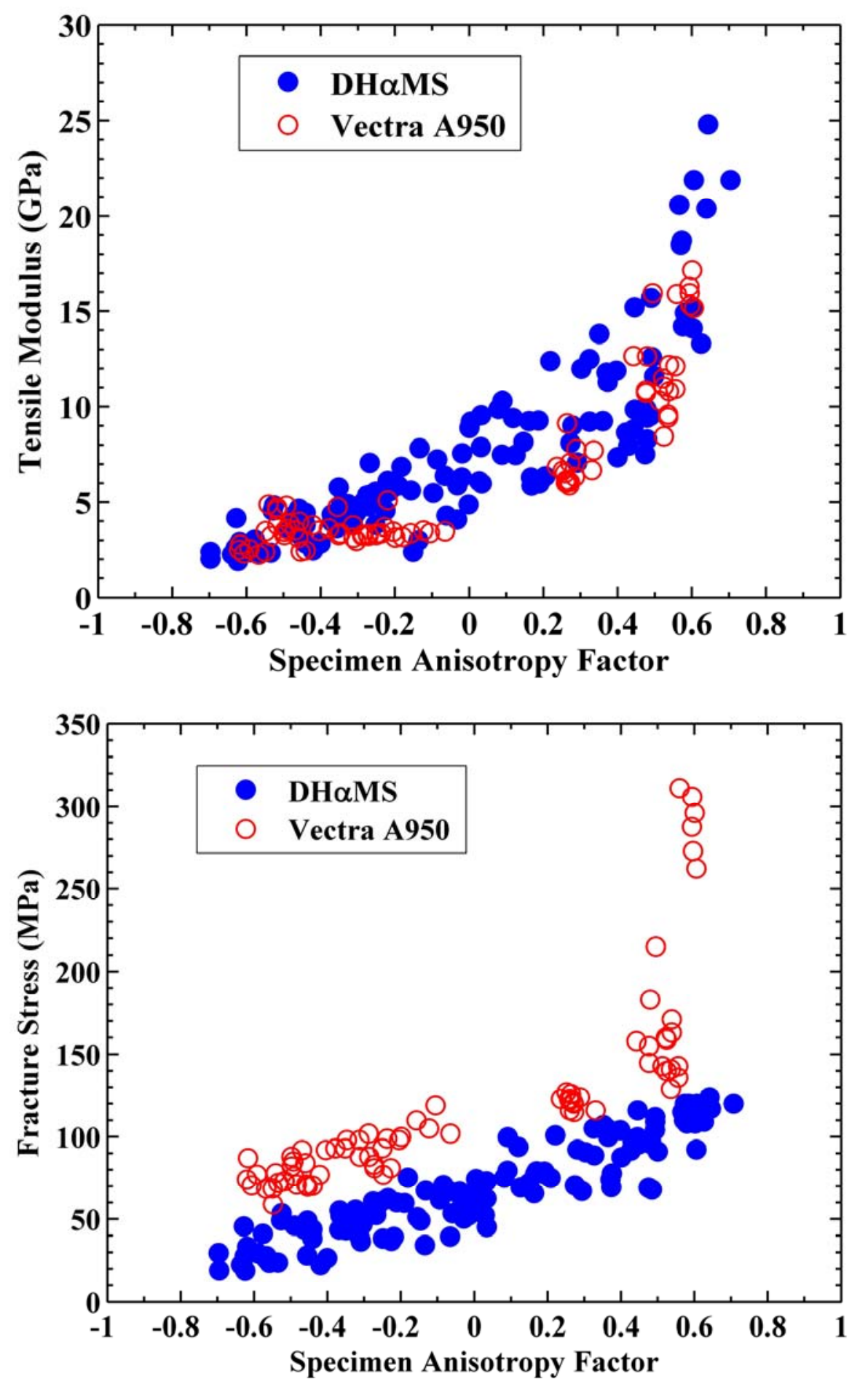

Figure 5. Completed master plots of tensile modulus and fracture stress as a function of anisotropy factor $(A F)$ for ASTM tensile specimens cut parallel and perpendicular to the principle flow direction of DHaMS and Vectra ${ }^{\circledR}$ A950 plaques fabricated under similar range of processing conditions. 

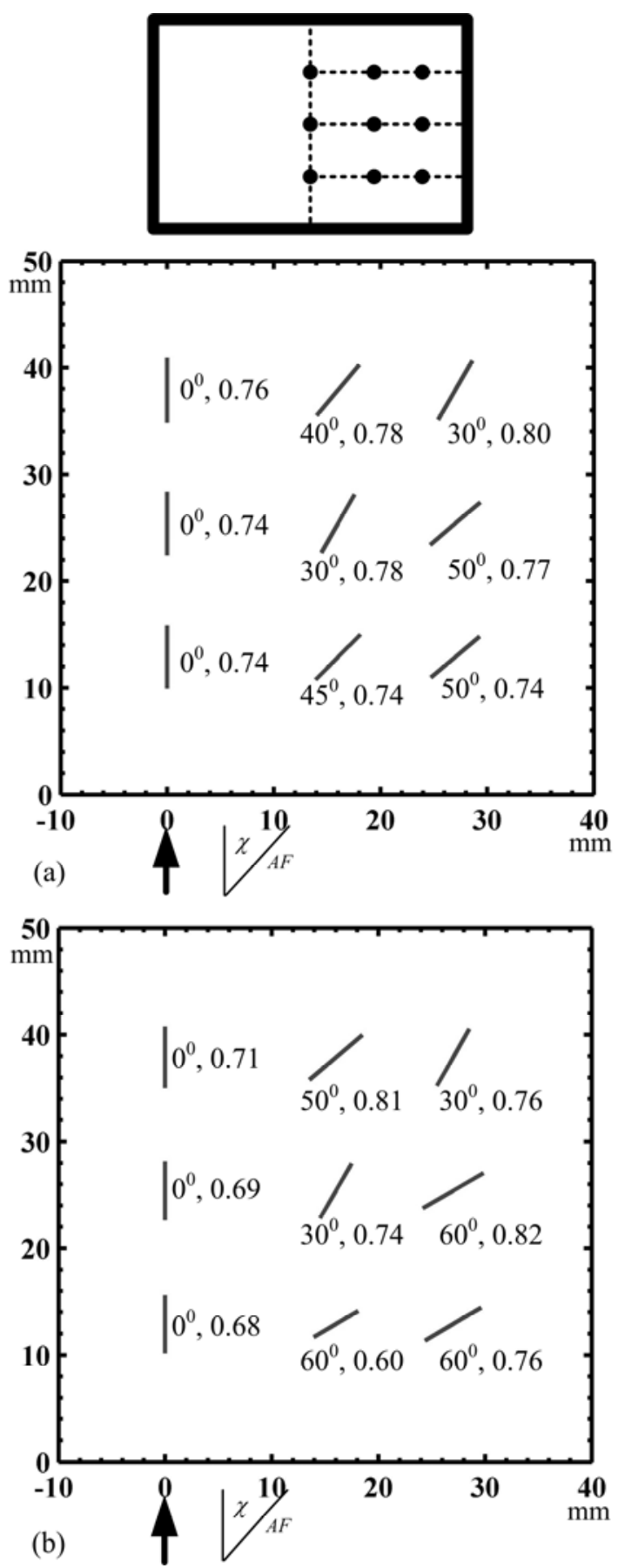

Figure 6. Vector plot and quantitative comparison on near surface orientation states measured via (a) NEXAFS and (b) ATR-FTIR. Measurements were taken on the right half of the plaque $(72 \mathrm{~mm} \mathrm{x}$ $50.8 \mathrm{~mm}$ ) where symmetry was assumed to either side of the centerline, as defined by the fill location (gate). The position of the narrow gate is noted by the arrow on the bottom edge of the Vectra ${ }^{\circledR}$ A950 plaque. The azimuthal angle for maximum orientation $(\chi)$, orientation parameter $(S)$, and orientation function $(f)$ are indicated for each of nine positions in (a) and (b), respectively. The positive direction of angles is measured clockwise from the primary flow direction. Estimates of uncertainty in orientation parameter values are \pm 0.02 and \pm 0.015 for NEXAFS and FTIR-ATR, respectively; uncertainty in orientation angle is estimated to be $\pm 5^{\circ}$. [8] 

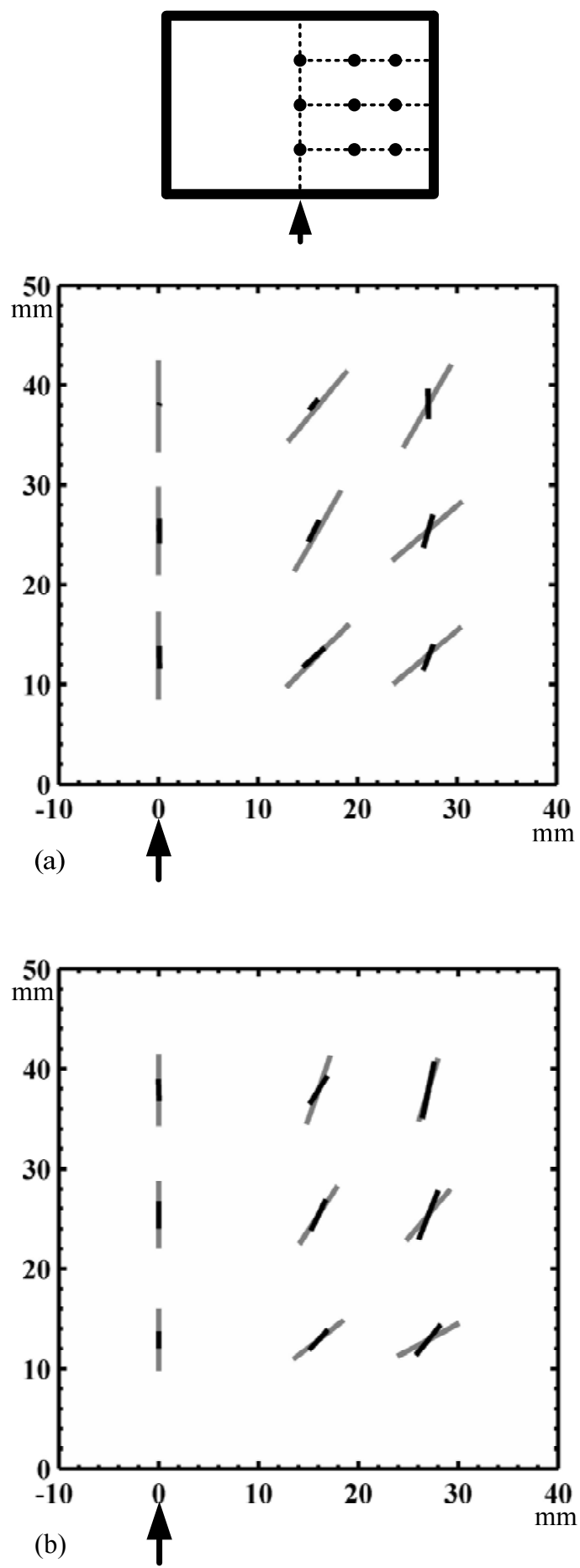

Figure 7. Vector plot and quantitative comparison on near surface orientation states measured via two complementary analytical techniques, (a) NEXAFS and (b) ATR-FTIR from Figure 6 shown in gray with the results of the modified Moldflow simulation superimposed. The position of the narrow gate is noted by the arrow on the bottom edge of the Vectra ${ }^{\circledR}$ A950 plaque. The azimuthal angle for maximum orientation $(\chi)$, orientation parameter $(S)$, and orientation function $(f)$ are indicated for each of nine positions in (a) and (b), respectively. 


\section{REFERENCES}

[1] A. M. Donald, A. H. Windle, and S. Hanna, Liquid Crystalline Polymers, Cambridge University Press, Cambridge (2006).

[2] Liquid Crystalline Polymers, E.T. Samulski, ed., National Materials Advisory Board, National Academy Press, USA, NMAB-453 (1990).

[3] S. Dreher, S. Seifert, H.G. Zachman, N. Moszner, P. Mercoli, and G. Zanghellini, J. Appl. Polym. Sci. 67531 (1998).

[4] D.K. Cinader and W.R. Burghardt, J. Polym. Sci.: Part B: Polym. Phys. 37, 3411(1999).

[5] 1989 Annual Book of ASTM Standards, 08.01, Plastics, pp 156 - 176, ASTM, Philadelphia, PA.

[6] J. Stöhr and M.G. Samant, J. Elec. Spectrosc. Relat. Phenom. 98-99 189 (1999).

[7] L. R. Pattison, A. Hexemer, E. J. Kramer, P. M. Petroff, and D. A. Fischer, Macromol. 39, 2225 (2006).

[8] S. Rendon, R. A. Bubeck, L. S. Thomas, W. R. Burghardt, A. Hexemer, and D. A. Fischer; J. Applied Polym. Sci., 21, 3445 (2007).

[9] A. Pirnia, and C. S. P. Sung, Macromol., 21: 2699 (1988).

[10] S. Besaad, B. Jasse, and C. Noël, Polym., 3481602 (1993).

[11] S. Rendon, W. R. Burghardt, A. New II, R. A. Bubeck, L. S. Thomas, Polym., 455341 (2004).

[12] W.R. Burghardt, V.M. Ugaz, D.K. Cinader, Scatt. Polym.739:374 (2000).

[13] R. A. Bubeck, L. S. Thomas, S. Rendon, W. R. Burghardt, A. Hexemer, and D. A. Fischer, J. Appl. Polym. Sci., 98, 2473 (2005).

[14] S. Rendon, W. R. Burghardt, R. A. Bubeck, L. S. Thomas, B. Hart, Polym., 4610202 (2005).

[15] S. K. Garg and S. Kenig, Ch. 3 in High Modulus Polymers, eds., A.E. Zachariades and R.S. Porter, Dekker, New York (1988).

[16] Ibid. [1].

[17] R. G. Larson, "The structure and rheology of complex fluids", Oxford University Press (1999).

[18] F. Folger, C. L. Tucker, Journal of Reinforced Plastic Composites, 3, 98 (1984).

[19] R. A. Nyquist, Private communication.

[20] I. Ouchi, M. Hosol, S. Shimotsuma, Journal of Applied Polymer Science, 21, 3445 (1977).

[21] R. A. Nyquist, "IR and NMR Spectral Data-Structure Correlations for the Carbonyl Group” Sadler Research Laboratories, Philadelphia, PA (1986).

[22] R. F. Larson and M. Doi, J. Rheol., 35, 539 (1991).

[23] J. Fang, W.R. Burghardt and R.A. Bubeck. "Bulk and Surface Molecular Orientation Distribution in Injection Molding of Thermotropic Liquid Crystalline Polymers: Simulation and Experiments." Submitted to Polymer Engineering \& Science, June 2009.

[24] J. Fang, W. R. Burghardt, and R. A. Bubeck, Polym. Eng. Sci., 48, 2258 (2008).

[25] J. L. Ericksen, Arch. Rat. Mech. Anal., 4, 231 (1960).

[26] C. A. Hieber, S. f. Shen, J. Non-Newton. Fluid Mech., 7, 1 (1980). 
Principal Investigator/Project Director: Dr. Robert A. Bubeck

Institution: Michigan Molecular Institute

Award Number: DMI-0521771

Program: MPM

Project Title: Microstructural Modeling and Synchrotron Studies of Orientation Development in Injection Molding of Liquid Crystalline Polymers

\title{
Comparative Determinations of Surface Orientation in Injection-Molded Thermotropic Liquid Crystalline Copolyester (TLCP) Plaques - Annual Report June, 2007
}

Robert A. Bubeck, Senior Research Scientist and Professor Michigan Molecular Institute, Midland, MI 48640

Wesley R. Burghardt (co-PI), Professor and Chair Department of Chemical and Biological Engineering Northwestern University

Evanston, IL 60208

\begin{abstract}
Injection molding thermotropic liquid-crystalline polymers (TLCPs) usually results in the fabrication of molded articles that possess complex states of orientation that vary greatly as a function of thickness and part geometry. This condition gives rise to "skin-core" morphologies and sample anisotropy that are often observed in TLCP moldings. Complementary mechanical property data for more than one TLCP for the sake of comparison are being obtained. In order to better understand how processing conditions influence anisotropy, samples were fabricated from three different commercial thermotropic liquid crystalline copolyesters. Attenuated Total Reflectance-Fourier Transform Infrared (ATR-FTIR) dichroism and Near-edge X-ray Absorption Fine Structure (NEXAFS) are being utilized in conjunction as means of determining surface layer orientation. The NEXAFS data was analyzed to determine the state of molecular orientation of the nematic polymers on the very near surface (depth of $\sim 3 \mathrm{~nm}$ ). These results are being used in combination with two-dimensional wide-angle X-ray scattering (2-D WAXS, depth of $\sim 0.5 \mathrm{~mm}$ ) in transmission and ATR-FTIR dichroism (depth of $\sim 5 \mu \mathrm{m}$ ) to characterize to various probe depths the orientation in the "skin" and "core" regions of the injection-molded plaques. These results, along with the mechanical property determinations, will be used to guide the development of improved modeling of the processing of TLCPs within the framework of the Moldflow ${ }^{\circledR}$ software package during the latter stage of the project.
\end{abstract}




\section{INTRODUCTION}

Thermotropic liquid crystalline polymers (TLCPs) combine the virtues of superior tensile properties with the ability to injection mold with very easy flow through the spontaneous ordering of molecules. These attributes are key to the use of these materials in high-performance electrical connectors for which the attainment of high physical properties in one dimension is a principal requirement. A critical processing issue is the development of high anisotropy during TLCP processing. Although the concurrence of high tensile properties and high directional orientation is of great benefit in fiber spinning, severe anisotropy can be a plague in obtaining balanced properties in net-shape injection-molded parts. The rigid nature of the mesogenic segments in TLCP molecules usually leads directly to a high orientational bias favoring the direction of flow with injection molding and other directional processing of thermotropes. ${ }^{1,2}$ Upon recrystallization, the high molecular orientation often leads to very favorable properties in the direction of orientation and lower physical properties in the transverse direction.

The high specific strength of TLCPs makes them excellent potential candidates for the manufacture of strong lightweight net-shape parts intended for use in transportation applications. The singular challenge in affecting their use in this manner is gaining control over the severe anisotropy that can often result during melt processing. Towards this end, our research is targeted at: (1) improved understanding of how processing conditions influence orientation and physical properties; and (2) using the results to develop new modeling strategies for injection-molding to predict orientation formation based upon a so-called 'polydomain' description of orientation which facilitates a direct connection to experimental measurements of orientation. An important part of this research effort is an extensive 3-D mapping of orientation in fabricated parts processed under defined conditions with model TLCPs. Progress in this effort is presented in this report.

Characterizations were previously performed for the direction of greatest strength in relatively narrow injection-molded tensile bars fabricated from Celanese Vectra ${ }^{\circledR}$ 6-hydroxy-2-naphthoic acid/6-hydroxybenzoic acid (HBA/HNA) type copolyesters by Dreher, et al. ${ }^{3}$ These researchers 
showed that tensile modulus increases linearly with the Hermans orientation function. Molding a plaque or other part with a broad aspect ratio, however, will incur much greater flow complexity and, therefore, more complex states of orientation. Shear flow dominates near the surface while transverse stretching dominates near the mid-plane ${ }^{4}$ resulting in bimodal cross-ply orientation, as schematically shown in Figure 1.

Depending upon thickness, a "skin/core" structure also results with a high molecular alignment in the 'skin' that may be maximized in a direction different from that in the "core". Shear asserts increasing dominance with decreasing sample thickness. Accordingly, the emphasis in this study concerns the molding of plaques under a systematic set of conditions and thicknesses, and then characterizing the resulting bimodal orientations.

Near-edge X-ray absorption fine structure (NEXAFS) was used to determine the molecular orientation in the near surface ( $2 \mathrm{~nm}$ deep). Two-dimensional wide-angle X-ray scattering (2-D WAXS) in transmission was used to determine average orientations of the "skin" and "core" layers and their directions of greatest magnitude by performing, when necessary, deconvolution of the scattering associated with the bimodal contributions from the "skin" and "core" layers. The NEXAFS results were augmented by surface orientation determinations made by Attenuated Total Reflectance - Fourier Transform Infrared (ATR-FTIR) dichroism. The results from NEXAFS and ATR-FTIR were then compared to define the state of surface orientation in the plaques. These results along with other orientation determinations made by 2-D wide-angle X-ray scattering (2DWAXS) will ultimately be used to guide the development of improved modeling of the processing of TLCPs within the framework of the Moldflow ${ }^{\circledR}$ software package during the latter stages of the project.

\section{EXPERIMENTAL}

\section{2. a. Materials}

Three thermotropic liquid crystalline polymers are being utilized in this study. The first is a copolyester containing 4,4'-dihydroxy- $\alpha$-methylstilbene (DHaMS) as the mesogen and a 
terephthalate/isophthalate/2,6-naphthalenedicarboxylate molar ratio of 65/10/25. The second polymer under study is Ticona Vectra ${ }^{\circledR}$ A950, which is a random copolyester consisting of $73 \%$ hydroxybenzoic acid (HBA) and 27\% 2,6-hydroxynaphthoic acid (HNA). On a limited basis, some experimentation has been performed using Ticona Vectra ${ }^{\circledR}$ B900 composed of $60 \%$ HNA, 20\% terephthalic acid (TA), and 20\% aminophenol (AP). Both Vectra resins were supplied by Ticona. Polymers of molecular weights in the environs of about 35,000 g/mol were evaluated.

\section{2. b. Reprocessing DH $\alpha$ MS TLCP}

In order to fulfill the critical need for more DH $\alpha$ MS TLCP for project activities, resin that had been previously supplied by Dow Chemical was taken through a multi-step process that resulted in pelletized material of high quality. The material was supplied to MMI in block and flake form. The reason for pelletizing these materials was to put them into a form that could be readily studied by in-situ synchrotron-based experiments and be injection molded into test plaques. It was found earlier that the flake form could not be successfully molded due to problems feeding the low bulk density flake into the plasticating barrel of the injection-molding machine.

Some of the lots of DHaMS were in large block form. These were roughly $2 \times 2 \times 2$ inch cubes that had been cut from extruded patties of polymer. These were size reduced into low bulk density flake at MMI used the Nelmor, model G810PT, unit. Extrusion into pellets was done at B\&P Process Equipment and Systems, 1000 Hess St., Saginaw, MI 48601. Their 18mm co-rotating twin-screw laboratory machine was used, as shown in Figure 2. The facility was equipped with vacuum venting for removal of volatiles including water, a volumetric feeder for the flake, a twohole strand die, a water bath for cooling extruded strands, and a Sheer-Bay, model BT-25, table top

pelletizer. The screw configuration was selected to optimize melt homogenization and devolatilization. The screw length/diameter ratio was 25/1. The average run conditions for the three lots of resin processed are contained in the following Table I. 
Table I. Parameters for Reprocessing DH $\alpha$ MS TLCP.

\begin{tabular}{|c|c|c|c|c|c|c|c|}
\hline Lot No. & $\begin{array}{c}\text { Melt Temp. } \\
\left({ }^{\circ} \mathrm{C}\right)\end{array}$ & $\begin{array}{c}\text { Rate } \\
(\mathrm{lb} / \mathrm{hr})\end{array}$ & $\begin{array}{c}\text { Screw } \\
\text { Speed } \\
(\mathrm{rpm})\end{array}$ & $\begin{array}{c}\text { Discharge } \\
\text { Pressure (psi) }\end{array}$ & $\begin{array}{c}\text { Torque } \\
(\%)\end{array}$ & $\begin{array}{c}\text { Vacuum } \\
(\mathrm{in} / \mathrm{Hg})\end{array}$ & $\begin{array}{c}\text { Amount } \\
\text { Extruded } \\
(\mathrm{lb})\end{array}$ \\
\hline 1 & 262 & 14.4 & 418 & 137 & 39 & 28 & 33.5 \\
\hline 2 & 260 & 7.0 & 540 & 60 & 20 & 29 & 13.5 \\
\hline 3 & 265 & 14.3 & 487 & 110 & 31 & 28 & 25.1 \\
\hline
\end{tabular}

A particular lot (1) was most difficult to feed into the extruder due to a larger size flake and a lower bulk density compared to the two other lots. A total amount of high quality pelletized polymer equal to $72 \mathrm{lb}$. was made available by the reprocessing. The general color appearance of the pelletized material was quite good with very acceptable the pellet geometry with few fines that fed well into a molding machine.

\section{2. c. Fabrication}

Sample plaques were fabricated using a Boy $30 \mathrm{~T} 2$ injection molding machine with which molding parameters were controlled. The plaques measured $76.2 \mathrm{~mm} \times 76.2 \mathrm{~mm}$ and were molded with a coat hanger gate (Figure 3). The mold was made by Master Precision Mold Technology, Inc. (Greenville, MI) and was fitted with an insert with a polished face that permitted the fabrication of plaques of various thicknesses. These were selected to be $0.8 \mathrm{~mm}, 1.6 \mathrm{~mm}$, and $3.2 \mathrm{~mm}$. Typically, the total cycle time was $47 \mathrm{sec}$, the screw speed was $264 \mathrm{rpm}$, pack (holding) pressure was $280 \mathrm{bar}$ $\left(2.8 \times 10^{7} \mathrm{~Pa}\right)$, and the cooling (hold) time was $16 \mathrm{sec}$. Injection fill times of $1 \mathrm{sec}$ and $5 \mathrm{sec}$ were studied and these two times were obtained by varying injection pressure alone to the two values of $1391 \operatorname{bar}\left(1.39 \times 10^{8} \mathrm{~Pa}\right)$ and $140 \mathrm{bar}\left(1.40 \times 10^{7} \mathrm{~Pa}\right)$, respectively. In addition to a range of melt temperatures, two mold temperatures $\left(90^{\circ} \mathrm{C}\right.$ and $\left.45^{\circ} \mathrm{C}\right)$ were studied.

It was ascertained that the samples required surface cleaning to remove contaminants before the NEXAFS and ATR-FTIR dichroism examination. Cleaning was performed with a Bransonic 220 ultrasonic cleaner for duration of 5 min. using a 1\% solution of Alconox Liqui-Nox ${ }^{\circledR}$ cleaning agent in de-ionized water. Both the initial presence of contaminants and their effective removal were verified for selected samples by atomic force microscopy using a Topometrix 2000 AFM. 


\section{2. d. Tensile Bar Fabrication and Testing}

Micro-tensile bars were machined from the plaques in directions both parallel and perpendicular to the nominal flow direction using a computer programmed end mill. The milling machine was programmed to cut three identical tensile samples parallel to or four samples perpendicular to the principal flow direction. Thus, the middle sample parallel to flow was perfectly aligned to the direction for principal flow and, therefore, maximum orientation. The micro-tensile bars were tested at a deformation rate of $1.27 \mathrm{~mm} / \mathrm{min}$ using an Instron Model 1125 test frame with MTS ReNew(C) Upgrade Package equipped with an MTS Model 632.29E30 Option 012 extensometer. The standard ASTM D-638 "Standard Test Method for Tensile Properties of Plastics" 5 was used as a guide, but tensile testing procedures were adapted to the specimen dimensions adopted for this study. An average of a minimum of five replicates was reported as the final value.

\section{2. e. Near-Edge X-ray Absorption Fine Structure (NEXAFS)}

The contribution to the orientation of the skin layer is difficult to deconvolute from that of the core of a molding. Dreher and coworkers ${ }^{3}$ determined skin orientation in TLCP moldings using WAXS of microtomed layers. A less laborious and less intrusive means of determining surface orientation presents itself in the form of near edge X-ray absorption fine structure (NEXAFS). NEXAFS is a synchrotron source UV spectroscopy technique that is sensitive to the orientation of phenyl groups in the direction of melt flow via the intensity ( $I$ ) of the partial electron yield (PEY) of Auger electrons of $1 \mathrm{~s} \rightarrow \pi^{*}$ transition of the $\mathrm{C}=\mathrm{C}$ bonds in the $\mathrm{C} \mathrm{K}$ edge. The technique using PEY is sensitive to the top $2 \mathrm{~nm}$ of a surface. The orientation is determined through a series of measurements of the $\mathrm{C} \mathrm{K}$ edge spectrum over a range of incident angles, $\theta,\left(20^{\circ}, 30^{\circ}, 40^{\circ}, 55^{\circ}\right.$, $60^{\circ}, 70^{\circ}, 80^{\circ}$, and $90^{\circ}$ ) of a monochromated linearly polarized UV beam relative to the sample surface. This technique has been successfully used to determine the orientation of LC alignment on rubbed polyimide substrates by Stöhr and Samant ${ }^{6}$ and by Pattison et al. ${ }^{7}$

The NEXAFS was performed on the NIST/Dow Soft X-ray Materials Characterization Facility, U7A, at the National Synchrotron Light Source, Brookhaven National Labs. Using the technique of Stöhr and Samant, the $P E Y$ intensity $I(\theta)$ is predicted to take the form, 


$$
I(\theta)=A+B \sin ^{2} \theta
$$

regardless of the degree of orientation. Based on the method by Kramer recently published by Pattison et al., ${ }^{8}$ the molecular orientation parameter, $S_{\text {Surface, }}$ was calculated using:

$$
S_{\text {Surface }}=\left(1-\frac{2(A+B)}{A+\frac{B}{6 P}(3 P-1)}\right)
$$

where, $P$ is the beam polarization ( 0.85 in this case). An extensive discussion of the application of NEXAFS to the measurement of surface orientation in TLCPs and the presentation of an extensive range of results is covered by Rendon et al. ${ }^{8}$

\section{2. f. Attenuated Total Reflectance - Fourier Transform Infrared (ATR-FTIR) Dichroism}

Layer orientation in injection molded plaques of 6-hydroxy-2-naphthoic acid/6-hydroxybenzoic acid (HBA/HNA) (58 mol \% HBA and $42 \mathrm{~mol} \% \mathrm{HNA}$ ) random type copolyesters was determined by Pirnia and Sung ${ }^{9}$ using Fourier Transform Infrared (FT-IR) Attenuated Total Reflection (ATR) dichroism. This technique enabled these researchers to determine relative orientation encompassing a depth of material $5 \mu \mathrm{m}$ below the sample surface. Using measured dichotic ratios to calculate the orientation parameter, the skin, intermediate layers and core were characterized for a series of samples cut from positions along injection-molded plaques by progressively removing material by milling. A study of the surface orientation of injection-molded VECTRA B900 samples by a similar means was reported by Besaad, Jasse, and Noël. ${ }^{10}$

For the work presented here, the samples were analyzed by infrared spectroscopy using the Seagull ${ }^{\circledR}$ Variable Angle Reflectance Accessory in a Nicolet Nexus 670 FTIR spectrometer. The Seagull was equipped with an ATR Rotator, a ZnSe ATR crystal and a Wire Grid Polarizer. The polarizer was set for s-polarization and the Seagull was set for an incident angle of $55^{\circ}$. This incident angle was selected to be slightly above the experimental critical angle for the samples 
examined. Each sample was oriented on the sampling stage of the ATR Rotator so its cut edges corresponded to the $0^{\circ}$ and $90^{\circ}$ degree settings. A background spectrum was recorded using the clean ATR crystal and then sample spectra were collected with the ATR Rotator set to $0^{\circ}, 45^{\circ}$, and $90^{\circ}$. The spectra collected were signal averaged over $32 \mathrm{scans}$ at $8 \mathrm{~cm}^{-1}$ resolution, over a spectral range from $4000 \mathrm{~cm}^{-1}$ to $650 \mathrm{~cm}^{-1}$. Considerable effort was expended to resolve instrumental issues and assure the reproducibility of the data. Having an inventory of $0.8 \mathrm{~mm}$ thick samples previously characterized using NEXAFS and 2-D WAXS was of great benefit in accomplishing this task. It was found that the use of a calibrated torque wrench to tighten the sample back plate of the Seagull ZnSe ATR crystal stage to obtain optimum crystal-sample contact is critical in obtaining good results. The spectra were converted to absorbance units and baseline corrected. From these data, the dichroic ratios were determined using the $1502 \mathrm{~cm}^{-1}$ absorption band associated with in-plane aromatic ring bending. Orientation parameters $(S)$ were calculated from the dichroic ratios, as described by Pirnia and Sung. ${ }^{9}$

\section{2. g. 2-D WAXS Data and Analysis Procedures of Injection-Molded Plaques}

An orientation map for each plaque type intended for tensile testing was determined by 2-D WAXS performed at $20 \mathrm{keV}(0.62 \AA$ Á) for multiple positions on the samples using the 5BM beam line of the DuPont/Northwestern University/Dow Collaborative Access Team at the Advanced Photon Source of Argonne National Lab. An incident X-ray beam with a $1 \mathrm{~mm}$ diameter size was used. A Mar CCD detector was used to collect $512 \times 512$ pixel raw X-ray scattering patterns with 15-second exposures. The WAXS patterns were obtained in positions corresponding to a grid, points for which correspond to the critical positions for the tensile sections of the tensile samples machined from the plaques. There are significant bimodal contributions from both the 'core' and the 'skin' in the WAXS patterns obtained for the thicker plaques, an example of which is shown in Figure 3.

Details of the data analysis techniques employed are given by Rendon, et al. ${ }^{11}$ The following section contains a simplified summary of the scheme outlined in Figure 4. The 2-D WAXS patterns usually indicate the presence of two discrete populations of orientation: one parallel to the flow direction and the other transverse to the flow direction. As evidenced in all of the patterns, 
nematic crystalline peaks always diffract perpendicular to the orientation direction. Scans made along this horizontal region of the plaque suggest the dominance of transverse orientation modes relative to shear-flow modes manifested by strong crystalline peaks, which are indicative of higher degrees of molecular orientation. Away from the centerline position, the bimodal character switches direction indicating that the transverse mode has rotated relative to molecular orientation at the centerline.

An assumption of uniaxial orientation is not appropriate when studying complex flow kinematics where both shear and extensional flows occur across the entire thickness of the sample. Instead, a measurement of anisotropy in the scattering pattern was obtained by computing the azimuthal intensity scan at $q^{*} .{ }^{12}$ This is done by taking a point on the azimuthal scan represented by the unit vector $\mathbf{u}$, such that $u_{1}=\cos \beta$ and $u_{2}=\sin \beta$, where $\beta$ is the characteristic azimuthal angle. Anisotropy can then be described by computing a weighted average of the second moment tensor of $\mathbf{u}$.

$$
\langle\mathbf{u u}\rangle=\left[\begin{array}{ll}
\left\langle u_{1} u_{1}\right\rangle & \left\langle u_{1} u_{2}\right\rangle \\
\left\langle u_{1} u_{2}\right\rangle & \left\langle u_{1} u_{2}\right\rangle
\end{array}\right]=\left[\begin{array}{ll}
\left\langle\cos ^{2} \beta\right\rangle & \langle\sin \beta \cos \beta\rangle \\
\langle\sin \beta \cos \beta\rangle & \left\langle\sin ^{2} \beta\right\rangle
\end{array}\right]
$$

In this case $\langle\cdots\rangle$ represents an average weighted by the azimuthal intensity distribution $I(\beta)$, where $\beta$ is measured from the flow direction, as shown in Figures 3 and 4. For instance, the average $\left\langle\cos ^{2} \beta\right\rangle$ is given by the expression:

$$
\left\langle\cos ^{2} \beta\right\rangle=\frac{\int_{0}^{2 \pi} \cos ^{2} \beta I(\beta) d \beta}{\int_{0}^{2 \pi} I(\beta) d \beta}
$$

An anisotropy factor $(A F)$ is then computed by taking the difference in the principal eigenvalues of the second moment tensor given as follows:

$$
A F=\lambda_{1}-\lambda_{2}=\sqrt{\left(\left\langle u_{1} u_{1}\right\rangle-\left\langle u_{2} u_{2}\right\rangle\right)^{2}+4\left\langle u_{1} u_{2}\right\rangle^{2}}
$$


In the limit of perfect orientation, independent of direction, this quantity will yield a value of 1, while 0 reflects a randomized orientation state (i.e. isotropic). The average orientation direction is computed from the eigenvectors or principal directions of the second moment tensor.

Anisotropy measurements made along the centerline region of the plaque will simply amount to taking the difference in the diagonal components of equation $1,\left\langle u_{2} u_{2}\right\rangle-\left\langle u_{1} u_{1}\right\rangle=\left\langle\sin ^{2} \beta\right\rangle-\left\langle\cos ^{2} \beta\right\rangle$. Symmetry dictates that the off-diagonal components of the tensor be zero. For this investigation, a coordinate system was adopted in which the 1-axis is oriented along the prevailing shear-flow direction. Consequently, values of the Anisotropy Factor $(A F)$ that approach +1 indicate perfect molecular orientation along the prevailing flow direction, while values approaching -1 correspond to perfect orientation transverse to the flow direction.

\section{2. h. Determination of Skin Orientation from the 2-D WAXS Data.}

Quantitative determination of skin, core, and bulk degrees of molecular orientation in molded plaques involved computing order parameters from azimuthal intensity scans extracted from 2D WAXS patterns collected in transmission. Because 1D azimuthal scans contain information averaged through the entire plaque sample thickness, "skin" and "core" contributions to the orientation must be computed separately, as shown in Figure 4. Herman's order parameters that reflect the localized 'skin' contribution to the bulk molecular orientation are computed from 1D azimuthal intensity scans that only contain peak information due to shear flow. Similarly, localized 'core' contributions to the bulk molecular orientation are computed from azimuthal scans which only contain peak information due to transverse extension. These azimuthal scans are generated by applying a Gaussian deconvolution method (using a software platform known as Peakfit ${ }^{\circledR}$ ) to the original "bulk" azimuthal intensity scan that effectively generates two different sets of intensity peaks (i.e., two new azimuthal scans) each containing information from either shear or transverse modes of orientation. Having done this, it is assumed that the uniaxial orientation to compute the corresponding order parameters can be based upon the Herman's convention, viz.,

$$
S=\frac{1}{2}\left(3 \cos ^{2} \beta-1\right)
$$


where in the limit of perfect orientation $S$ has a value of 1 , and 0 indicates a randomized orientation state. The procedures are described further by Bubeck and co-workers. ${ }^{9,13}$

\section{RESULTS AND DISCUSSION}

\section{3. a. Mechanical Properties and Their Correlation to Orientation Determined by 2-D WAXS.}

Tensile properties versus molecular orientation for $76 \mathrm{~mm}$ by $76 \mathrm{~mm}$ injection molded plaques is represented by tensile modulus and fracture stress versus $A F$ for the range of processing conditions and thicknesses studied and is plotted in Figure 5. The newly obtained data for the Vectra A950 samples is superimposed on data for the DH $\alpha$ MS TLCP, which has been reported previously by Rendon et al. elsewhere. ${ }^{14}$ The samples with net negative values for $A F$ are those dominated by transverse extension during their molding while the samples with net positive values for $A F$ are those dominated by shear flow. The latter are populated principally by samples cut from the thinner plaques. The observed sharp increase in modulus with increasingly positive $A F$ is similar to and is consistent with what was indicated by data for the melt-drawing of HBA/HNA random copolyester TLCP filament by Garg and Kenig ${ }^{15}$, as plotted after the fact by Donald and Windle. ${ }^{16}$ Fracture stress was found to generally increase in a linear fashion with increasing $A F$ as well.

The data obtained for Vectra A950 to date indicates similar trends for tensile modulus, but elevated values vis-à-vis the DH $\alpha$ MS TLCP for fracture stress (i.e., the Vectra A950 is the tougher of the two polymers). The tensile data for the lower magnitude $A F$ values for the central portions of the plots has been obtained and the requisite 2-D WAXS data to complete the comparisons is planned to be completed later this year (2007).

\section{3. b. Examples of NEXAFS and ATR-FTIR Results for Surface Orientation.}

NEXAFS and ATR-FTIR are two independent means of determining surface orientation. The ATR-FTIR dichroism measurements were undertaken to supplement or sometimes be substituted 
for the synchrotron-based NEXAFS measurements, and to yield additional information resulting from a technique that probes to a much greater depth from the surface ( $5 \mu \mathrm{m}$ vs. $2 \mathrm{~nm}$ ) than the capability of $\mathrm{C} \mathrm{K}$ edge PEY NEXAFS. Example results for these two techniques along with through thickness results for 2-D WAXS for $0.8 \mathrm{~mm}$ thick $76 \mathrm{~mm}$ x $76 \mathrm{~mm}$ coat hanger gated plaques injection-molded with DHaMS and Vectra A950 are shown, respectively, in Figures 6 and 7. It has been previously demonstrated that the through-thickness orientation is unidirectional in the direction of flow along the center line for the $0.8 \mathrm{~mm}$ thickness because the polymer flow in the plaques under discussion is dominated entirely by shear flow with almost no transverse extensional flow. ${ }^{8,13}$ Consequently, the change in orientation with depth from the surface is indicated by the orientation parameters $\left(S_{\text {Surface }}\right)$ obtained by the two surface techniques. The 2-D WAXS in transmission yields values of $(S)$ that are through thickness averages and are much more heavily influenced by the orientation in the interior "core" region.

The results for the two polymers are similar in kind, but with some notable differences. As might be expected, the orientation at the very surface for a $2 \mathrm{~nm}$ depth via NEXAFS is the greatest and somewhat less for the ATR-FTIR dichroism results from probing to a $5 \mu \mathrm{m}$ depth. The thickness average $(S)$ obtained by 2-D WAXS is markedly less than either surface technique. A significant decrease in the through thickness average $S$ at the vent end (position \#4) of the plaques was observed to occur for both polymers. The greatest difference in $S$ as determined by NEXAFS at a $2 \mathrm{~nm}$ depth and ATR-FTIR at a $5 \mu \mathrm{m}$ depth was observed at the gate end for both TLCPs.

An example comparison of ( $\left.S_{\text {Surface }}\right)$ obtained by NEXAFS for positions 1 through 4 along the center line of $76 \mathrm{~mm} \times 76 \mathrm{~mm} \times 1.6 \mathrm{~mm}$ thick plaques injection molded from Vectra A950 and Vectra B900 is shown in Figure 8. The B900 is the more readily oriented under identical process conditions and this is reflected, as well, in the comparison of mechanical properties shown in Figure 9 for tensile samples obtained at the center line. The B900 tensile samples have the much greater moduli and lower \% strain values for fracture in the regime defined by melt temperatures of $285^{\circ} \mathrm{C}$ to $300^{\circ} \mathrm{C}$ and a mold temperature of $45^{\circ} \mathrm{C}$. 


\section{SUMMARY}

The influences of molding parameters and mold thickness on the anisotropy, skin-core morphology, and mechanical properties of injection molded DHaMS copolyester and Vectra thermotropic liquid crystalline polymers are being studied. Key observations and milestones for this year include:

1. The physical properties of commercially available Vectra A950 are influenced qualitatively by processing parameters in a fashion similar to that for the DHaMS copolyester; however, there are differences in their relative magnitudes and dependencies on anisotropy. Complementary 2-D wide-angle X-ray scattering measurements are underway to complete the determination of the anisotropy factors $(A F)$ that correspond to the measured properties. Under similar processing conditions, the properties of Vectra B900 (with its more rigid molecular structure) are markedly different than for the other two TLCPs. These attributes and relative differences will need to be taken into account as the modifications to and evaluations of processing modeling (e.g., Moldflow ${ }^{\circledR}$ ) are undertaken.

2. A Herrick Seagull ${ }^{\circledR}$ Variable Angle Reflectance Attenuated Total Reflection Fourier Transform Infrared (ATR-FTIR) dichroism accessory was procured and protocols for its use in the determination of surface orientations were accomplished. With some noted differences, trends in "skin" orientation were found to parallel those obtained by near edge X-ray absorption fine structure (NEXAFS) and ATR-FTIR. Magnitudes for orientation parameter $(S)$ were found to order themselves in relation to the relative depths to which each technique samples with NEXAFS yielding the greatest values of $S$ and 2-D WAXS the least and with ATR-FTIR in between. The equivalency of NEXAFS with ATR-FTIR techniques as measures of surface orientation was found to be very good when analyses of the NEXAFS data are accomplished using the Kramer scheme. $S_{\text {Surface }}$ is typically about $5 \%$ to $10 \%$ greater, as measured by NEXAFS. Adoption of ATR-FTIR dichroism permits the surveying of a wide range of sample "skin" orientations in house beyond what is facilitated by relatively limited amounts of available synchrotron beam time at national facilities.

3. Completion of extensive NEXAFS data analyses was accomplished (and published) based upon the Kramer scheme of determining near surface orientation. Up to now, a limited 
number of areas off-axis from the centerline of plaques has been interrogated by using small squares of material cut from the plaques of interest. With a new 6-axis manipulator now being made operational at the U7A end station, we intend to broadly map the orientation of selected $76 \mathrm{~mm}$ x $51 \mathrm{~mm}$ narrow-gated plaques. The narrow-gated plaques are of greater interest than the coat hanger gated plaques because of their flow complexity. A proposal for new beam time has been submitted to the National Synchrotron Light Source to accomplish this task. Cross correlation of results with ATR-FTIR dichroism measurements is planned.

\section{ACKNOWLEDGEMENTS}

First of all, thanks to my principal collaborators in this work, W. Burghardt and J. Fang (Northwestern University). The collaborative efforts with L. Thomas and K. Robertson (M.M.I.) are invaluable. The thoughtful and careful determination of physical properties by B. Hart and H. Fondriest were most helpful. Thanks are extended to J. Quintana and the staff of the DND-CAT of the Advanced Photon Source, Argonne National Labs; and to D. Fischer and the staff of Beamline U7A of the National Synchrotron Light Source, Brookhaven National Labs. Funding support from the National Science Foundation and the Department of Energy through NSF Grant DMI-0521771 is gratefully acknowledged. The gift of 55 lbs. of Vectra ${ }^{\circledR}$ A950 to our effort by Ticona is greatly appreciated.

Vectra ${ }^{\circledR}$ is the trademark of Ticona, a business of Celanese, Inc.

Seagull ${ }^{\circledR}$ is a trademark of Herrick Scientific products, Inc.

Moldflow ${ }^{\circledR}$ is a trademark of Moldflow, Inc. 


\section{FIGURES}

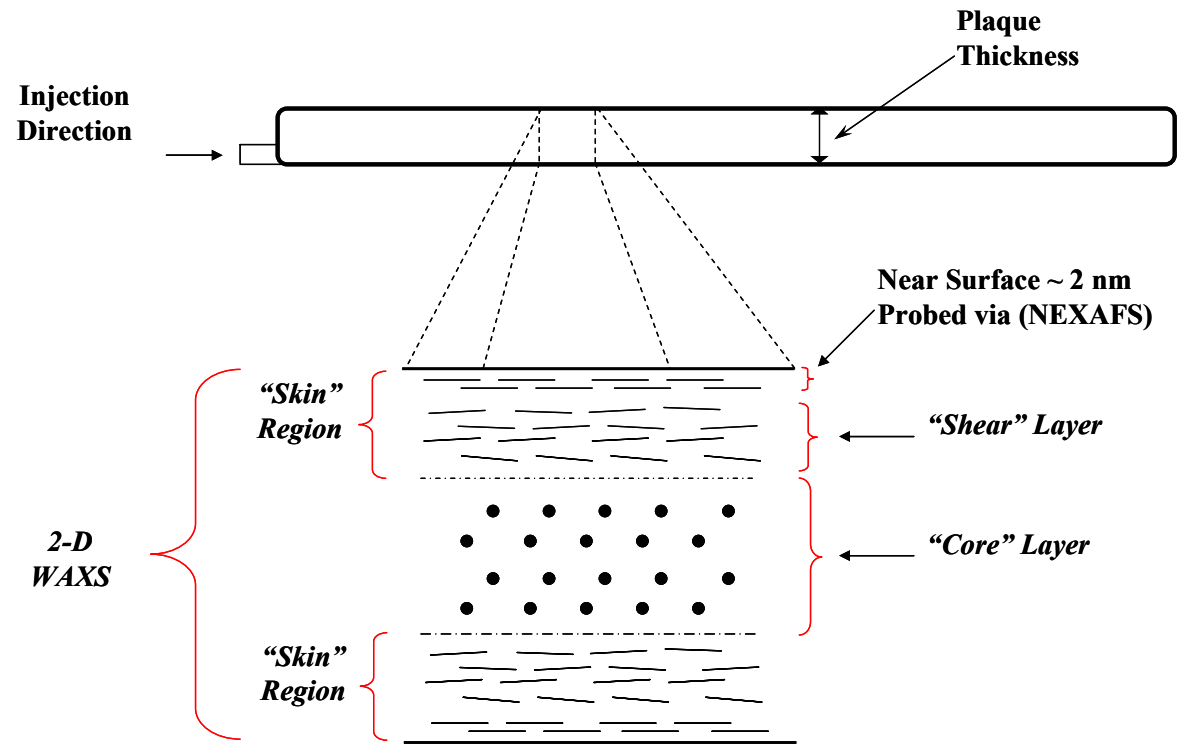

Figure 1. Idealized schematic of an injection molded plaque cross-section illustrating the levels of morphology present along the plaque thickness direction. 2-D WAXS in transmission averages structural information through the sample thickness while NEXAFS probes molecular orientation over depths of 2 to $3 \mathrm{~nm}$ in the near surface layer.

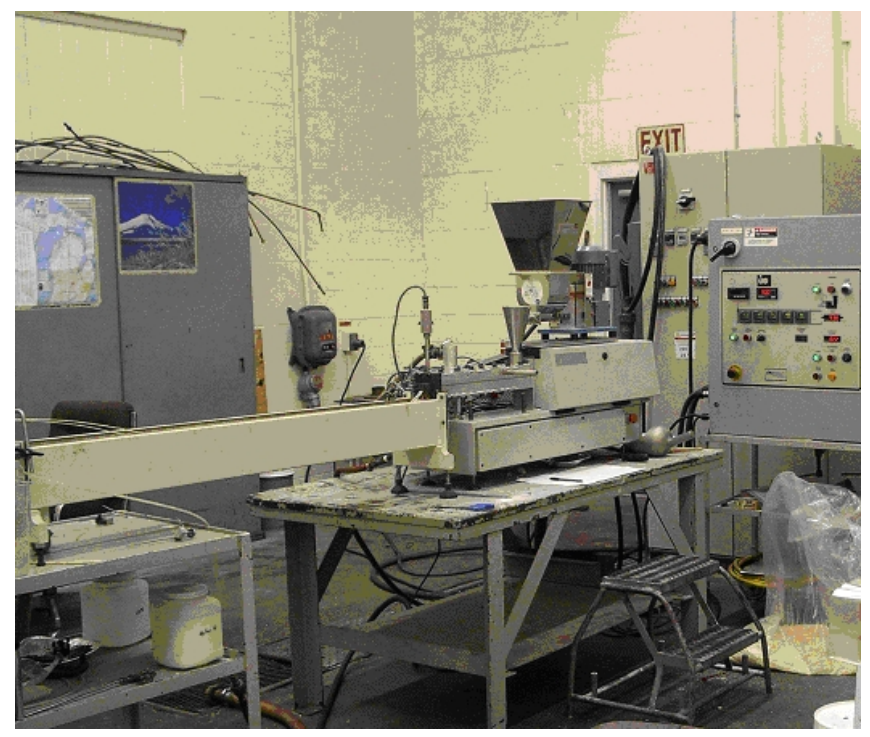

Figure 2. B\&P Process Equipment $18 \mathrm{~mm}$ co-rotating twin screw laboratory extruder and cooling water trough. 


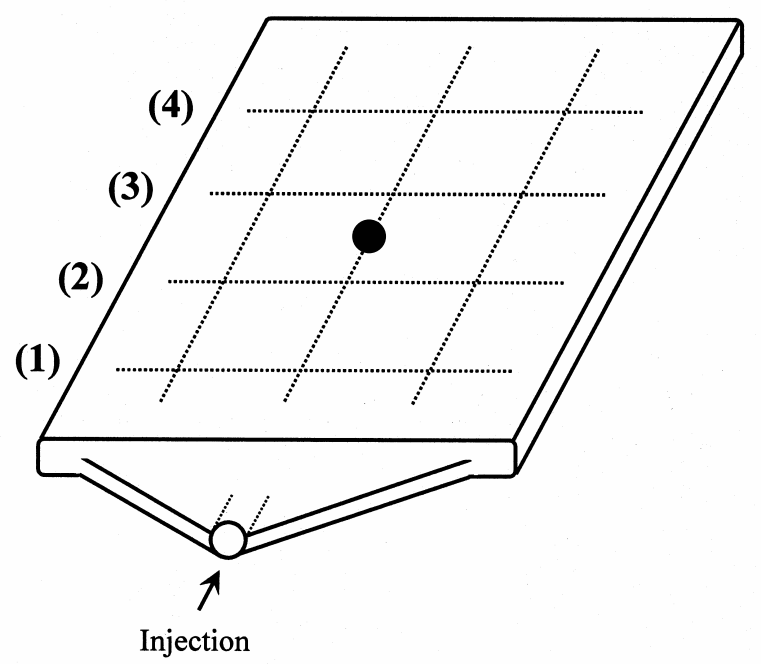

(a)

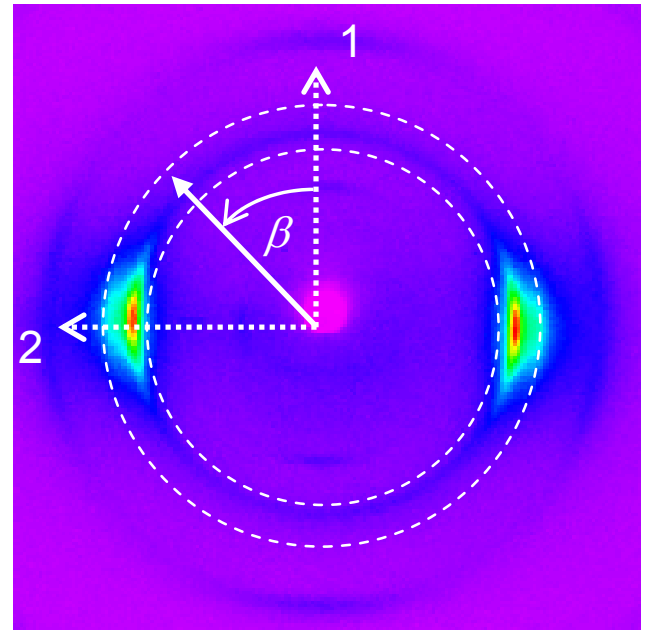

(b)

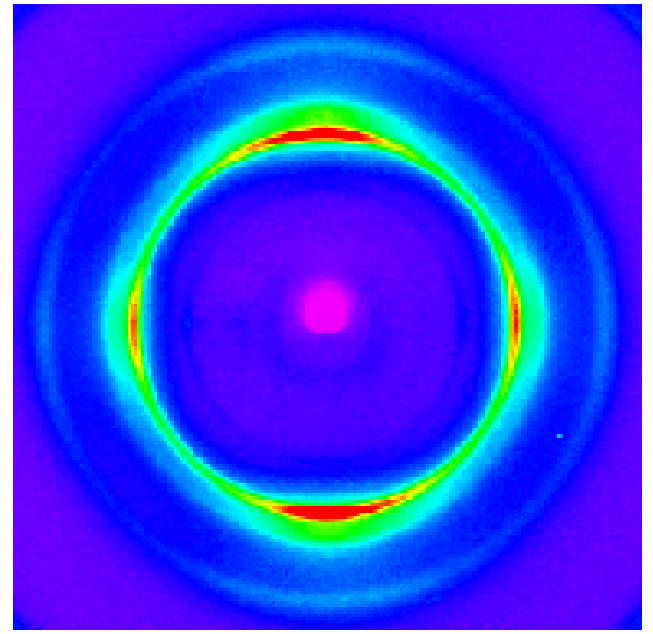

Figure 3. Examples of physical orientation of an injection-molded plaque along with two representative 2-D WAXS patterns taken for a centerline position of a: (a) $0.8 \mathrm{~mm}$ thick molded plaque showing uniaxial contribution to the orientation due to shear flow and a (b) $3.2 \mathrm{~mm}$ thick plaque showing bimodal orientation populations due to shear and extension. The arrow indicates the principal direction of flow. The plaques were processed at constant fast fill time of $1 \mathrm{~s}$ with melt and mold temperatures of $270^{\circ} \mathrm{C}$ and $45^{\circ} \mathrm{C}$, respectively. The overlays in (a) define the coordinates and range of the scattering wave vector, $q$, used for the extraction of azimuthal scans; $\beta$ is the azimuthal angle measured away from the vertical filling direction. Dotted lines indicate the path locations where 2-D WAXS measurements were performed $15 \mathrm{~mm}$ apart. 




Figure 4. Data analysis procedure used to extract azimuthal intensity scans from the scattering contributions due to shear ('skin') and extension ('core'). Two $I(\beta)$ scans are extracted from a single 1-D 'bulk' sample azimuthal scan via a Gaussian deconvolution of azimuthal intensity peaks. The scans are processed to compute $S_{\text {Shear }}$ and $S_{\text {Core. }}$ 

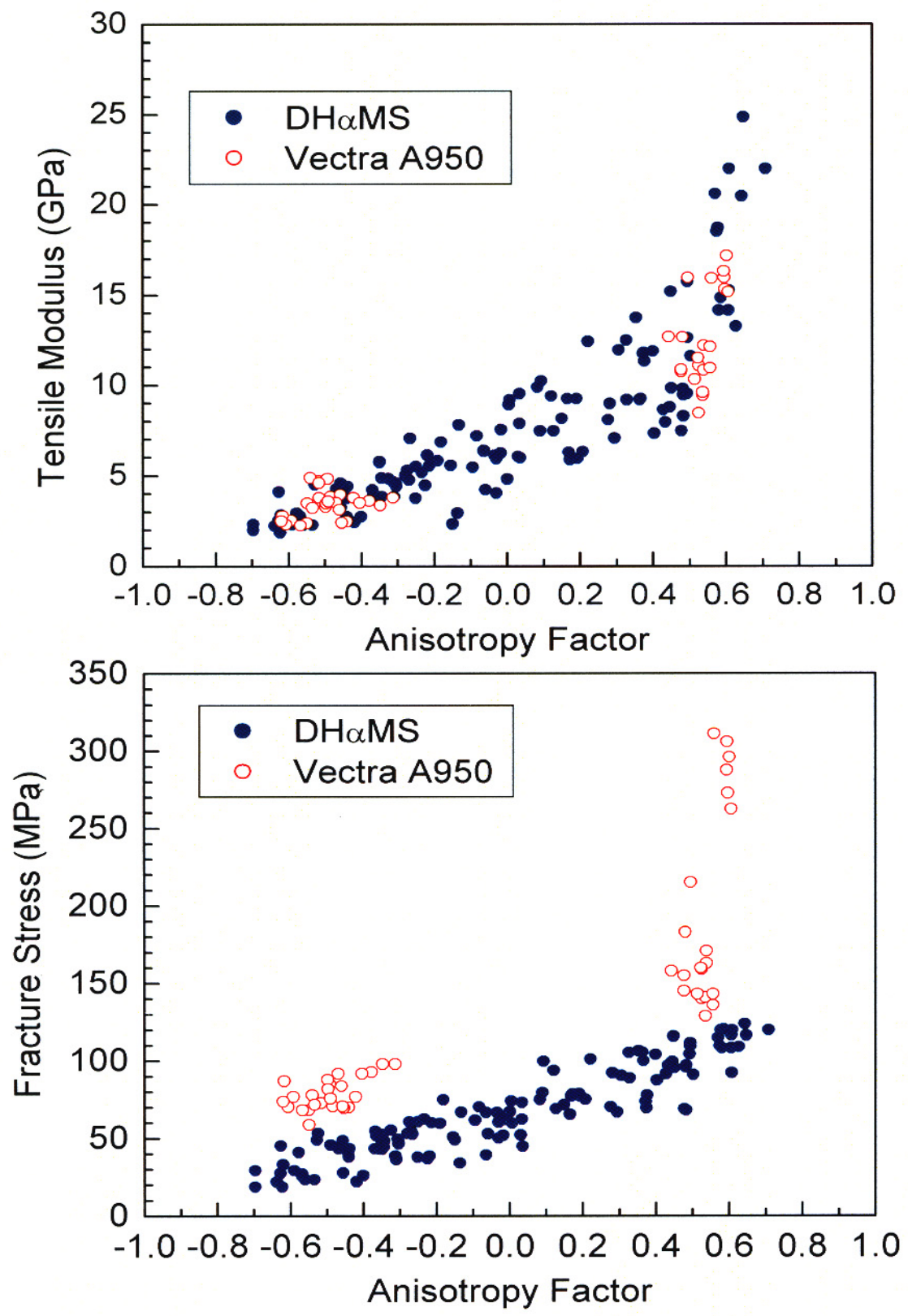

Figure 5. Master plots of tensile modulus and fracture stress as a function of anisotropy factor $(A F)$ for ASTM tensile specimens cut parallel and perpendicular to the principal flow direction of DHoMS and Vectra A950 plaques fabricated under similar range of processing conditions (Tensile data for Vectra A950 under conditions relevant to the central area of the plot (Anisotropy Factor, $A F=-0.4$ to 0.4 ) have been completed and the corresponding 2-D WAXS data is to be obtained during the third quarter of 2007). 


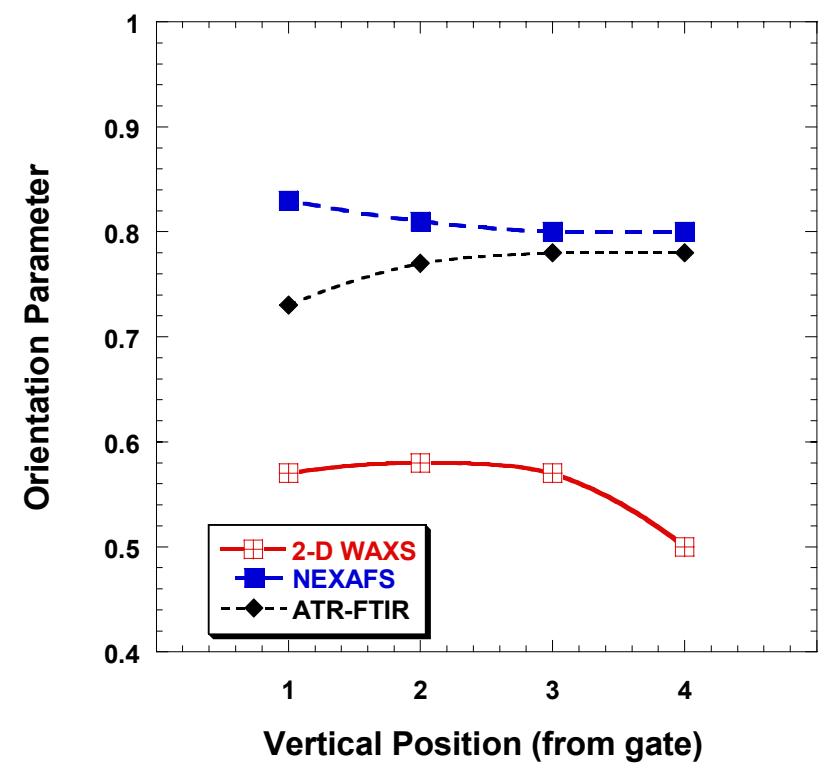

Figure 6. Example orientation parameters $(S)$ obtained via NEXAFS, ATR-FTIR, and 2-D WAXS, which probe to depths of about $2 \mathrm{~nm}, 5 \mu \mathrm{m}$, and $\sim 0.5 \mathrm{~mm}$, respectively. The data were obtained for four positions along the center line (corresponding to Figure 3) of an injection-molded DHaMS TLCP $76 \mathrm{~mm} \times 76 \mathrm{~mm} \times$ $0.8 \mathrm{~mm}$ thick coat hanger gate plaque processed with a $290^{\circ} \mathrm{C}$ melt temperature and $45^{\circ} \mathrm{C}$ mold temperature.

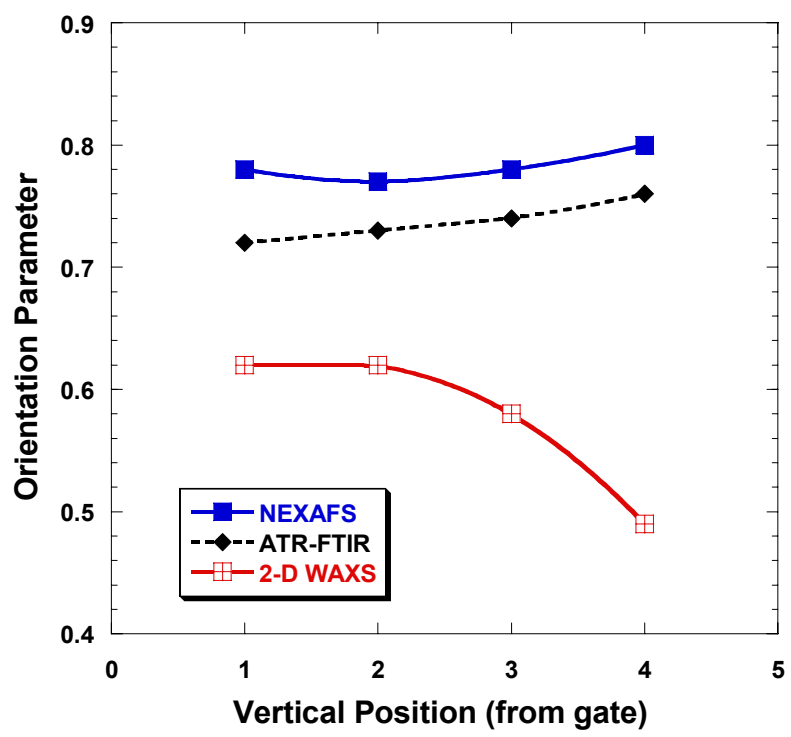

Figure 7. Example orientation parameter $(S)$ determinations obtained via NEXAFS, ATR-FTIR, and 2-D WAXS at four positions along the center line (corresponding to Figure 3) of an injection-molded Vectra A950 $76 \mathrm{~mm} \times 76 \mathrm{~mm} \times 0.8 \mathrm{~mm}$ thick coat hanger gate plaque processed with a $290^{\circ} \mathrm{C}$ melt temperature, $45^{\circ} \mathrm{C}$ mold temperature. 


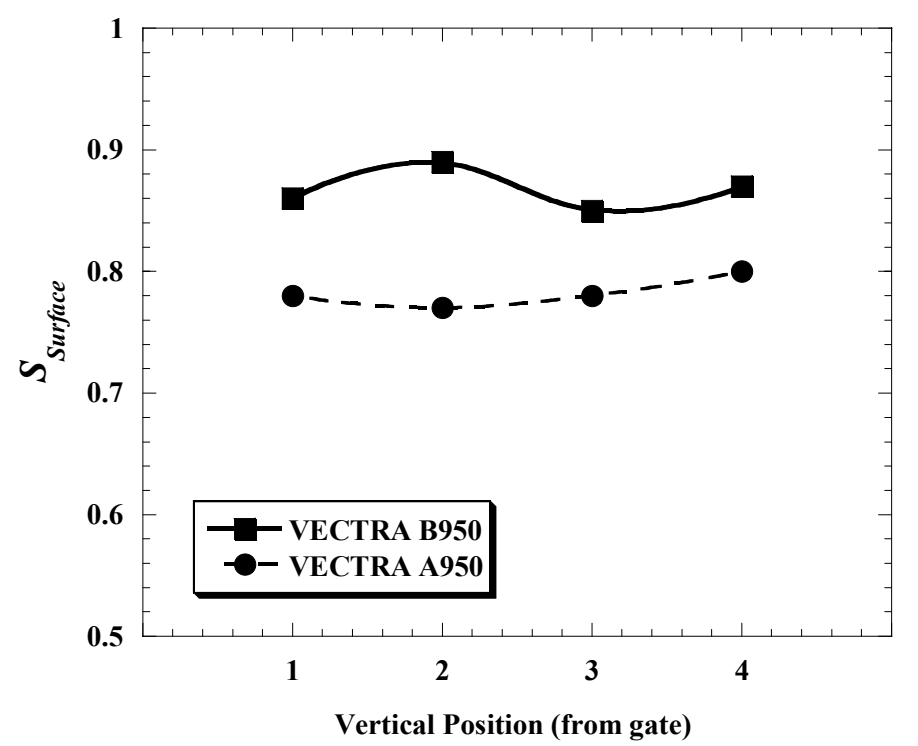

Figure 8. Comparative orientation parameters $\left(S_{\text {Surface }}\right)$ obtained using NEXAFS at four positions along the center line (corresponding to Figure 3) of injection-molded $76 \mathrm{~mm} \times 76 \mathrm{~mm} \times 1.6 \mathrm{~mm}$ thick coat hanger gate plaques of Vectra $\mathrm{A} 950$ and $\mathrm{B} 900$ processed with a $290^{\circ} \mathrm{C}$ melt temperature, $45^{\circ} \mathrm{C}$ mold temperature.

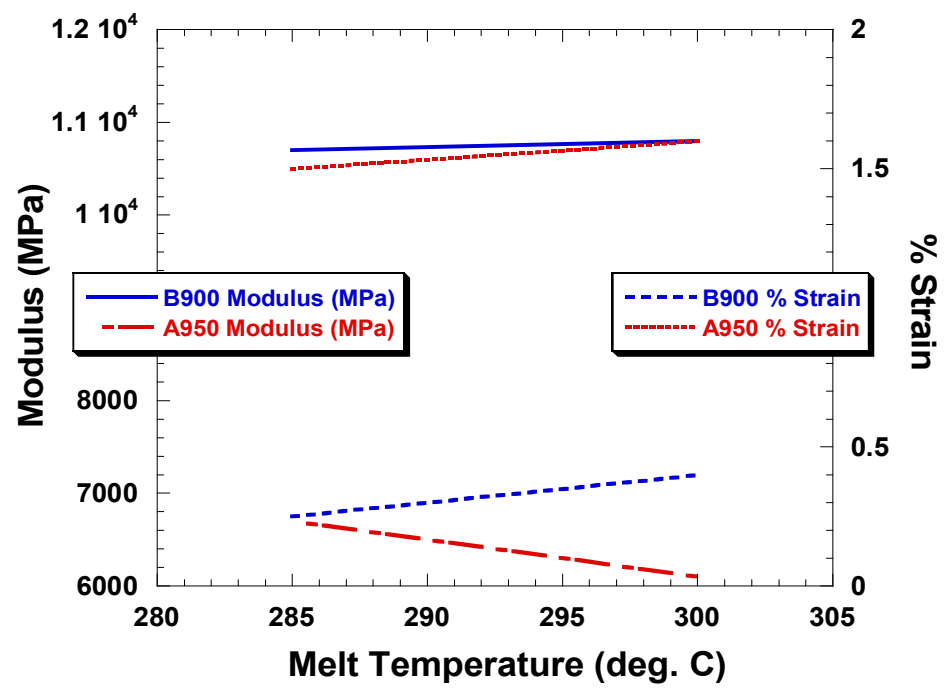

Figure 9. Comparative values for Tensile Modulus and \% Strain for Fracture corresponding to the middle region positions 2 and 3 of Figure 8 of injection-molded $76 \mathrm{~mm} \times 76 \mathrm{~mm} \times 1.6 \mathrm{~mm}$ thick coat hanger gate plaques of Vectra $A 950$ and $B 900$ processed with a $290^{\circ} \mathrm{C}$ melt temperature, $45^{\circ} \mathrm{C}$ mold temperature. The specimens were aligned parallel to the direction of flow at the center line of the plaque. 


\section{REFERENCES}

[1] A. M. Donald, A. H. Windle, and S. Hanna, Liquid Crystalline Polymers, Cambridge University Press, Cambridge (2006).

[2] Liquid Crystalline Polymers, E.T. Samulski, ed., National Materials Advisory Board, National Academy Press, USA, NMAB-453 (1990).

[3] S. Dreher, S. Seifert, H.G. Zachman, N. Moszner, P. Mercoli, and G. Zanghellini, J. Appl. Polym. Sci. $67531-545$ (1998).

[4] D.K. Cinader and W.R. Burghardt, J. Polym. Sci.: Part B: Polym. Phys. 37, 3411- 3428 (1999).

[5] 1989 Annual Book of ASTM Standards, 08.01, Plastics, pp 156 - 176, ASTM, Philadelphia, PA.

[6] J. Stöhr and M.G. Samant, J. Elec. Spectrosc. Relat. Phenom. 98-99 189-207 (1999).

[7] L. R. Pattison, A. Hexemer, E. J. Kramer, P. M. Petroff, and D. A. Fischer, Macromol. 392225 (2006).

[8] S. Rendon, R. A. Bubeck, L. S. Thomas, W. R. Burghardt, A. Hexemer, and D. A. Fischer; Journal of Applied Polymer Science, 2007, in press.

[9] A. Pirnia, and C. S. P. Sung, Macromolecules, 21: 2699 (1988).

[10] S. Besaad, B. Jasse, and C. Noël, Polymer 3481602 (1993).

[11] S. Rendon, W. R. Burghardt, A. New II, R. A. Bubeck, L. S. Thomas, Polymer, 455341 - 5352 (2004).

[12] W.R. Burghardt, V.M. Ugaz, D.K. Cinader, Scatt. Polym.739:374 (2000).

[13] R. A. Bubeck, L. S. Thomas, S. Rendon, W. R. Burghardt, A. Hexemer, and D. A. Fischer, Journal of Applied Polymer Science, 98, 2473-2480 (2005).

[14] S. Rendon, W. R. Burghardt, R. A. Bubeck, L. S. Thomas, B. Hart, Polymer, 46 $10202-10213$ (2005).

[15] S. K. Garg and S. Kenig, Ch. 3 in High Modulus Polymers, eds., A.E. Zachariades and R.S. Porter, Dekker, New York (1988).

[16] Ibid. [1]. 
Final Report for Period: 09/2008 - 08/2009

Principal Investigator: Baird, Donald G.

Organization: VA Polytechnic Inst \& St U

\section{Submitted By:}

Baird, Donald - Principal Investigator

Title:

Simulation of Injection Molding of Thermoplastics Reinforced with Fibers and Nano-Particles

\section{Senior Personnel}

Name: Baird, Donald

Worked for more than 160 Hours: Yes

Contribution to Project:

Name: Wapperom, Peter

Worked for more than 160 Hours: Yes

Contribution to Project:

\section{Project Participants}

Submitted on: 02/09/2009

Award ID: 0521918

\section{Post-doc}

\section{Graduate Student}

Name: Eberle, Aaron

Worked for more than 160 Hours: Yes

\section{Contribution to Project:}

This student was primarily concerned with assessing the appropriate choice of constitutive equation should be used, rheological measurements, and model parameter selection.

Name: Velez, Gregorio

Worked for more than 160 Hours: Yes

\section{Contribution to Project:}

This student worked with Professor Wapperom to develop finite element code incorporating the constitutive equations developed above and carried out fiber orientation studies at Oak Ridge National Laboratories.

Name: Ortman, Kevin

Worked for more than 160 Hours: Yes

\section{Contribution to Project:}

This student learned to carry out confocal laser microscopy measurements and understand and use the numerical code. He was supported by this grant.

\section{Undergraduate Student}

Name: Hughes, Gerald

Worked for more than 160 Hours: Yes

Contribution to Project:

Continued analyzing the fiber length distribution of various short and long glass fiber-filled composites. Aided in rheological measurements. Paid from grant on an hourly basis.

\section{Technician, Programmer}




\section{Other Participant}

\section{Research Experience for Undergraduates}

\section{Oak Ridge National Laboratories}

\section{Organizational Partners}

ORNL has facilities for measuring fiber orientation for comparison to the simulations.

\section{RTP}

RTP has provided a range of fiber reinforced polypropylenes including long and short fiber materials.

Professor Charles Tucker

\section{Other Collaborators or Contacts}

\section{Activities and Findings}

\section{Research and Education Activities:}

The goal of this work is to improve on current predictions of fiber orientation in polymer matrices using a constitutive relation in which fiber orientation is coupled with the flow and not post-calculated. The major research and education activities are:

1. To develop rheological techniques for handling fluids containing high aspect ratio fibers and to connect the rheological behavior to fiber orientation. Studies have been carried out to determine the impact of fiber concentration, aspect ratio, orientation distribution and suspending medium viscoelasticity on the rheology of short and long glass fiber composite fluids.

2. To teach numerical methods suitable for handling the flow of complex fluids. A finite element simulation was developed as discussed below.

3. Numerical schemes which give conversion at high Deborah numbers were addressed. A Code for a discontinuous Galerkin finite element method has been written that can handle multiple viscoelastic constitutive equations. Simulations have been carried out for shear/extensional rheometrical flows and 2-D injection molding flows with the Hele-Shaw approximation.

4. Rheometry in conventional rheometers is difficult especially for long fiber systems. A sliding plate rheometer was designed and built for studying the rheology and flow of long-glass fiber composites.

5. Methods for investigating of fiber orientation during flow were investigated. Center-gated samples were injection molded and analyzed for fiber orientation at Oak Ridge National Laboratories.

Further details are available in the attaced pdf file(report).

\section{Findings:}

In order to simulate short fiber orientation distribution accurately in injection molding it was found that the initial fiber orientation entering the mold cavity must be known. Assuming random fiber orientation is not adequate, as one must either measure the initial fiber orientation (which is what we did initially) or simulate the flow in the runner to predict it. The layered structure observed in fiber orientation of an injection molded part is due to this initial fiber orientation.

The prediction of fiber orientation in an injection molded part was improved significantly by incorporating non-affine fiber motion and coupling fiber orientation and flow, as compared to the existing approach which has been to decouple fiber orientation from flow. However, the predictions of fiber orientation still need to be improved, and the fiber orientation caused by extensional flow at the advancing front must be incorporated into the simulation (future effort).

The incorporation of fiber flexibility into the simulation package through the use of the bead-rod model showed qualitative agreement with measured fiber orientation for injection molded long-fiber composites. Fiber orientation of long fibers in the center of a part is significantly different than that observed for short fiber composites.

Sliding-plate rheometry is needed to characterize the rheology of long fiber composites as conventional methods are inadequate.

\section{Training and Development:}

The students involved in this project are learning: 1) how constitutive relations for polymers containing high aspect ratio fibers are developed; 
2) how to design rheological tests for assessing the parameters in the models; 3) how to develop finite element code for complex fluids in which structure and flow are coupled; 4) the importance of coupling simulations with the measurement of fiber orientation. More details can be found in attaced pdf file.

\section{Outreach Activities:}

We have incorportated some of the results from the rheological studies of fiber filled melts into a short course being taught to industrialists(on campus and then on-sight). We have given about 20 presentations to universities and at conferences pertaining to this research.

\section{Journal Publications}

A. P.R. Eberle, D. G. Baird, and P. Wapperom, "Modeling the Transient Rheology of a Polypropylene Melt Reinforced with Long and Short Glass Fibers", SPE Technical Papers, p. 1257, vol. 52, (2006). Published,

D. Baird and A. Eberle, "Modeling The Rheology And Orientation Distribution Of Short Glass Fibers Suspended In Polymeric Fluids: Simple Shear Flow", Proceedings of the 65th Annual Technical Conference, p. 2823, vol. 65, (2007). Published,

A. P. E. Eberle, D. G. Baird, and P. Wapperom, "The Rheological Properties of Non-Newtonian Fluids Containing Glass Fibers: A Review of Literature", Ind. Eng. Chem. Res., p., vol. , ( ). Submitted,

Eberle, A. P. R.; Baird, D. G.; Wapperom, P., "The rheology of non-Newtonian fluids containing glass fibers: A review of experimental literature", Ind. Eng. Chem. Res., p. 2849, vol. 47, (2008). Published,

A. P. R. Eberle, D. G. Baird, P. Wapperom, and G. M. Velez-Garcia, "Using Transient Rheology to Determine Material Parameters in Fiber Suspension Theory", J. Rheol., p. , vol. , (2009). Accepted,

A. P. R. Eberle, D. G. Baird, P. Wapperom, and G. M. Velez-Garcia, "Obtaining Reliable Transient Rheological Data on Concentrated Short Fiber Suspensions Using a Rotational Rheometer", j. Rheol., p. , vol. , (2009). Accepted,

A. P. R. Eberle, G. M. Velez-Garcia, D. G. Baird, and P. Wapperom, "Fiber Orientation Kinetics of a Concentrated Short Glass Fiber Suspension in Startup of Simple Shear Flow", J. Non-Newt. Fluid Mech., p. , vol. , (2009). Submitted,

\section{Books or Other One-time Publications}

\section{Web/Internet Site}

\section{Other Specific Products}

\section{Contributions within Discipline:}

Contributions

A procedure for identifying the appropriate rheological experiments for determing a unique set of rheological parameters in a constitutive equation for polymers containing long glass-fibers was developed. Furthermore, the equations of motion coupled with this constitutive equation have used to formulate a simulation of injection molding in which flow and fiber orientation are coupled. This has been done initially assuming Hele-Shaw flow but is presently being extended to general flow including the advancing front. Appropriate rheological tests were developed which provide the necessary material parameters needed in the numerical simulation.

\section{Contributions to Other Disciplines:}

The mechanical properties of an injection molded part are highly dependent on fiber orientation. The mechanical properties observed for parts generated in injection molding can now be better explained and predicted as a function of mold design.

\section{Contributions to Human Resource Development:}


Gregorio Velez is an instructor at the University of Puerto Rico. He is developing visualization and modeling techniques which can be taken back to his university to improve design of injection molding processes for polymer composites.

We have incorporated one undergraduate into the research effort in an attempt to get them interested in pursuing an advanced degree.

The graduate students involved in the project have learned the connection between basic rheology of complex fluids and simulation of processing.

\section{Contributions to Resources for Research and Education:}

The students have learned that the flow behavior must be connected to the structure and orientation of fibers in simple flows first before one can set out to model more complex flow situations. Computer simulations have been developed which will be available for use by undergraduates in their courses on process design.

\section{Contributions Beyond Science and Engineering:}

We are in the process of developing new software for designing molds used to produce parts consisting of plastics reinforced with long fibers. This software will eventually be an improvement over commercially available software. The simulation package will be available to a wide range of industries, such as the automotive industry, to accelerate the design of injection molding processes for making high performance and lightweight parts.

\section{Conference Proceedings}

\section{Any Book}

\section{Categories for which nothing is reported:}

Any Web/Internet Site

Any Product

Any Conference 
DOE/NSF February, 2009 Final Report

\title{
Simulating Injection Molding of Thermoplastics Reinforced with Short and Long Fibers
}

\author{
Principal Investigator: Donald G. Baird \\ Virginia Polytechnic Institute and State University \\ Department of Chemical Engineering \\ Blacksburg, VA 24061 \\ (540) 231-5998, fax: (540) 231-2732, email: dbaird@vt.edu \\ Co-Principal Investigator: Peter Wapperom \\ Virginia Polytechnic Institute and State University \\ Department of Mathematics \\ Blacksburg, VA 24061 \\ (540) 231-7252,email: pwappero@vt.edu
}

Participants

Aaron P. R. Eberle, PhD. student, email: eberle@vt.edu

Gregorio M. Velez,PhD. student, email: gvelez@vt.edu

Kevin C.Ortman,PhD. student, email: kco3@vt.edu

Contractor: Virginia Polytechnic Institute and State University

Contract No.: DMI-052918

\section{Objective}

- Improve the accuracy of the simulation of short (rigid) and long (flexible) fiber orientation in thermoplastics during injection molding by using a modified constitutive relation that incorporates non-affine motion and flexibility in combination with state-of-the-art numerical techniques.

- Establish a method (independent of the injection molding process) for determining an unique set of material parameters which is based on the transient shear rheological behavior of the composite fluid.

- Evaluate the accuracy of the simulation technique by comparing numerical predictions of glass fiber orientation to that first found in rheometrical flows and then in injection molded parts produced using basic mold geometries (end and center-gated parts).

\section{Approach}

- Determine an approach to conduct quantitative and reproducible rheological experiments on glass fiber-filled polymeric fluids.

- Conduct transient shear rheological studies on glass fiber-filled polypropylene (PP) and polybutylene terephthalate (PBT) systems in which fibers of various lengths (length: $0.2-11 \mathrm{~mm}$, diameter: 12.5 microns) are used to assess the effects of both fiber length and the viscoelastic nature of the matrix on the transient rheology.

- Experimentally characterize the relationship between the rheological behavior and the associated fiber microstructure.

- Identify the limitations of current approaches to predict the evolution of fiber orientation by comparing model predictions to experimental observations. 
- Use modified theory to address these limitations.

- Define and evaluate specific rheological tests to determine the material parameters in the constitutive equation which are unique and give consistent results when used in numerical simulations.

- Develop a finite element method simulation program capable of predicting fiber orientation during the mold filling stage of thermoplastic composite fluids containing short and long glass-fibers in an injection molding process.

- Modify the simulation to include the extensional flow kinematics of the advancing front region which may be critical to predicting fiber orientation at the mold wall.

- Assess the performance of the simulation by comparing predicted fiber orientation against values determined experimentally from injection molded samples.

\section{Accomplishments}

- Rheological Characterization Technique (short fibers): Developed a novel rheological technique that addresses problems associated with the non-homogeneous shear field found in parallel-plate rotational rheometers and boundary interactions in cone-and-plate rheometers.

- Rheological Characterization Technique (long fibers): A fully functional sliding plate rheometer incorporating a shear stress transducer has been designed and fabricated primarily for the purpose of performing unbiased and reproducible steady shear rheological experiments on long glass fiber-filled polymeric fluids that are otherwise impossible to perform on rotational rheometers. Moreover, the device has been calibrated, and its behavior has been verified using two reference materials, a viscosity standard and a commercial LDPE. In addition to rheological studies, the rheometer will also be used in tracking the transient evolution of long fiber orientation upon startup and cessation of shear flow.

- Rheology: Rheological characterization of the short glass fiber composites was performed, including intermittent stress growth/relaxation tests to elucidate the stress contribution of the fiber, matrix, and flow field on the transient evolution of fiber orientation. A novel approach using donut shaped samples in a coneand-plate rheometer was developed for tracking transient shear behavior of short-fiber composites.

- Model (short fibers): A constitutive relation has been developed that incorporates stress contributions from the fiber and the viscoelastic suspending medium and includes the ideas of non-affine motion. Model predictions show good agreement compared to rheological experiments and preliminary results for the experimentally determined fiber orientation.

- Fiber Orientation Measurements: We have determined an approach to characterizing the 3D fiber orientation within composite parts using confocal laser microscopy. Additionally, an improved method that uses optical microscopy was also developed, and was shown to provide a more economical means for acquiring orientation measurements. This latter method is, therefore, expected to be the chosen method for future acquisition. With these techniques, we have initiated the characterization of fiber orientation in both rheometrical flows and complex flow geometries.

- Model (long fibers): Various approaches to modeling flexible fibers have been investigated. These approaches include modifying current theory for rigid fibers to include a "flexibility parameter", exploring constitutive equations resulting from bead-rod theory, track individual fibers using Hinch's infinitely flexible fiber theory, and extending Hinch's equations for concentrated polymer solutions through the concept of mobility. The choice of approach is still not determined but will be the emphasis of future research. 
DOE/NSF February, 2009 Final Report

- Simulations (short): Developed a Galerkin/discontinuous Galerkin finite element simulation package to simulate the filling stage of injection molding. The features of this simulation package are: the use of multiple constitutive equations, the comparison with the Hele-Shaw approximation for an end-gated plaque and a center-gated disk, and a combined front tracking/ ALE technique for advancing front simulations.

- Simulations (long): Constructed a bead-rod orientation model for long fibers within the finite element simulation package. This model was used to gain preliminary results for long fiber orientation in injection molded center gated disks. Additionally, a program capable of utilizing an extension of Hinch's equation for concentrated polymer melts, through the use of a mobility tensor, was also written.

- Injection molding: A complete set of short and long glass fiber reinforced parts were molded, from which variables affecting fiber orientation and polymeric behavior (i.e. Newtonian-like/viscoelastic matrices, geometry, part thickness, and fiber length) are being studied.

\section{Future Direction}

- Compare model predictions of short glass fiber theory including non-affine motion to that of experimental results.

- Complete the analysis of orientation in center-gated disks and end-gated plaques using the alternate nonambiguous protocol for fiber orientation measurement in short glass fiber composites.

- Incorporate frontal flow in the simulation and compare to previous predictions using just Hele-Shaw approximation.

- Simulate fiber orientation in the runner of an injection mold cavity in order to establish the initial fiber orientation at the entrance to the cavity.

- Focus our attention on characterizing the transient rheological behavior of long glass fiber suspensions using the sliding plate rheometer.

- Use the sliding plate rheometer to gain an understanding of long fiber orientation development in simple shear experiments.

- Use the sliding plate rheometer to gain an understanding of fiber mobility in concentrated fiber suspensions and to assess the performance of Hinch's equation and its extensions.

- Assess the performance of the bead-rod model for various (long) fiber lengths by comparing model predictions to fiber orientation obtained in injection molded parts. 
DOE/NSF February, 2009 Final Report

\section{Introduction}

The overall goal of our research is to be able to accurately predict the orientation of short and long fibers suspended in polymer melts generated during injection molding using a finite element analysis. To accomplish this goal we first have to establish a constitutive relation capable of predicting both the stress response to deformation of a composite fluid and the associated microstructure. As discussed, our approach to accomplish this goal begins with comparing model predictions to the rheological behavior and experimentally determined microstructure in start-up of simple shear flow experiments. Furthermore, unique material parameters for a specific composite fluid are established by fitting to these rheological measurements. Once established, the constitutive equation, using the unique model parameters, is then implemented into a finite element code for complex flow simulations typically found in injection molding, i.e. center-gated and end-gated flow.

We will highlight our advancements in the following order. First, we will discuss a unique method to accurately quantify the transient rheological behavior of short glass fiber-filled composite fluids and update the reader on the development of the sliding plate rheometer. After this we will describe, in general, the transient rheological behavior of these fluids characterized using this technique. Then we will discuss the improvements/modifications made to the constitutive theory by incorporating the idea of non-affine motion and a parameter to control fiber flexibility. In addition we will discuss current theories used for the dynamic simulation of long/flexible fibers. We then compare our experimental results and model predictions for short glass fiber suspensions and describe our approach at determining unique material parameters by fitting to the transient shear stress in start-up of flow. Following, we will describe our approach to characterizing the evolving microstructure of short glass fibers in simple flows and compare these results to model predictions. Finally we will outline our progress in the finite element simulation of a center gated mold and describe our findings, i.e. the effect of initial conditions and non-affine motion.

\section{Experimental Procedure}

Rheological Characterization (Short fiber composite fluids): A key part of our research is to be able to quantitatively characterize the transient rheological behavior of glass fiber composite fluids to assess our modeling approach and determine unique material parameters. Historically, researchers have used the parallel plate or disk geometry in rotational rheometers to characterize the transient rheological behavior of short glass fiber-filled fluids to minimize boundary-fiber interaction. To reduce the degree of boundary interaction it has been suggested that the rheometer gap be at least three times the length of the longest dimension of the suspension particle. Experimental results using parallel plate geometry with various rheometer gaps confirmed that for the $30 \mathrm{wt} \%$ PBT there is a negligible effect on the sample rheological response when the gap is greater than twice the number average fiber length. The problem with this geometry (parallel plate) is the tangential velocity and hence, shear rate varies with radial position from the center of the plates to the outside. Our experimental results have shown that this inhomogenous shear field causes unrealistic transient behavior and, therefore, cannot be used as a quantitative tool. As a result, we believe only a rheometer that imposes a homogeneous shear field should be used to quantitatively characterize suspensions of glass fibers.

The gap within the $50 \mathrm{~mm}$ cone and plate fixture varies linearly from $2.51 \mathrm{~mm}$ at the outer edge to $0.05 \mathrm{~mm}$ at the center. To remove the inevitable fiber-boundary interaction near the center, sample disks were pre-formed and a $25.4 \mathrm{~mm}$ diameter hole was drilled through the center creating a donut shaped sample. A schematic of the "donut" sample can be seen in Fig. 1. These samples combined with the cone and plate geometry ensure a homogeneous shear field and a gap that was always greater than two times the number average fiber length(Complete details are given in ref. 3 below). 


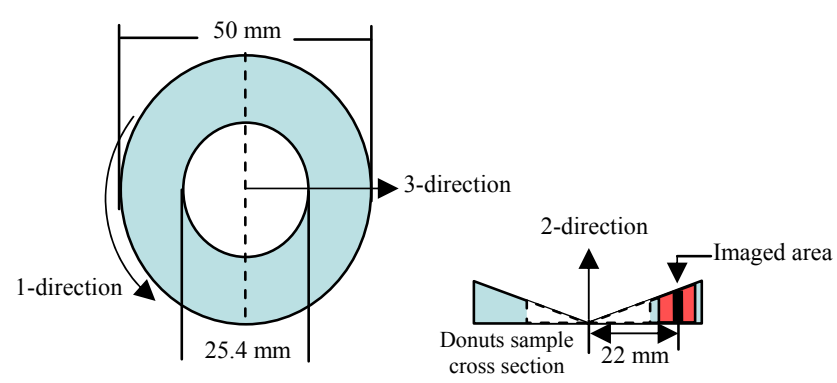

Figure 1. $50 \mathrm{~mm}$ cone and plate "donut sample" dimensions. The illustration defines the 1, 2, and 3directions which are the direction of flow, velocity gradient and radial directions respectively. In the cross sectional profile the black area is the area at which the confocal laser images were taken.

\section{Sliding Plate Rheometer (Characterization of long fiber composite fluids):}

After numerous attempts at characterizing suspensions containing long glass fibers using various techniques at our disposal, we concluded that the curvilinear streamlines and rheometer gap size to fiber length ratio (found in conventional melt rotational rheometers) was inadequate. As a result we initiated the design and fabrication of a sliding plate rheometer incorporating a wall shear stress transducer with the primary purpose being to perform unbiased and reproducible steady shear rheological experiments on long glass fiber-filled polymeric fluids that are otherwise impossible to perform on rotational rheometers. After completion the device was calibrated and its behavior was verified. A viscosity standard and a commercial polymer Equistar PETROTHENE NA 952, a LDPE with no processing aids or antiblock agents, were used as reference materials.

Localized stress measurement and a homogenous shear field with rectilinear streamlines are two key improvements the sliding plate rheometer offers over rotational rheometers. While localized stress measurement eliminates the problems arising due to edge effect, the homogenous shear field insures removal of unrealistic transient behavior. Moreover, theoretically it has no restriction on the gap height. Fig. 2 (a)-(c) are pictures of the sliding plate, wall shear stress transducer and disassembled wall shear stress transducer showing rigid beam. In addition to rheological studies, the rheometer will also be used in tracking the transient evolution of long fiber orientation upon startup and cessation of shear flow.



(a)

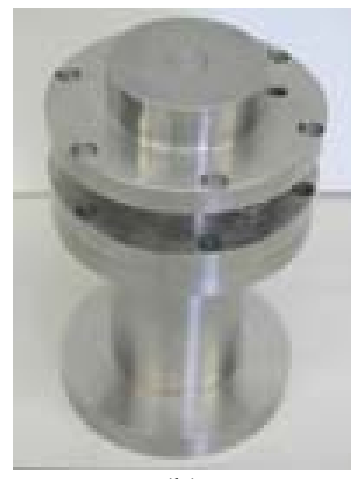

(b)

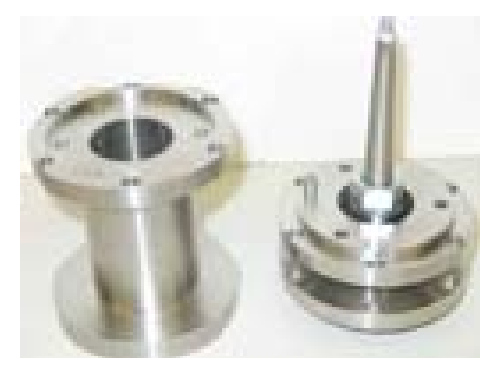

(c) 


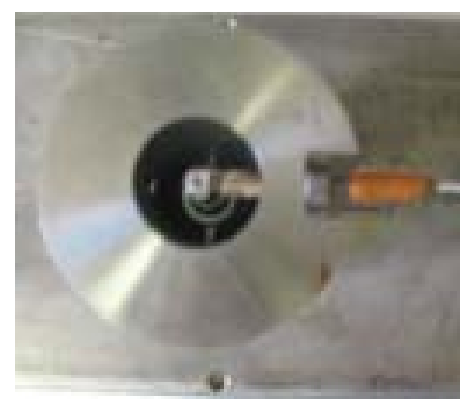

(d)

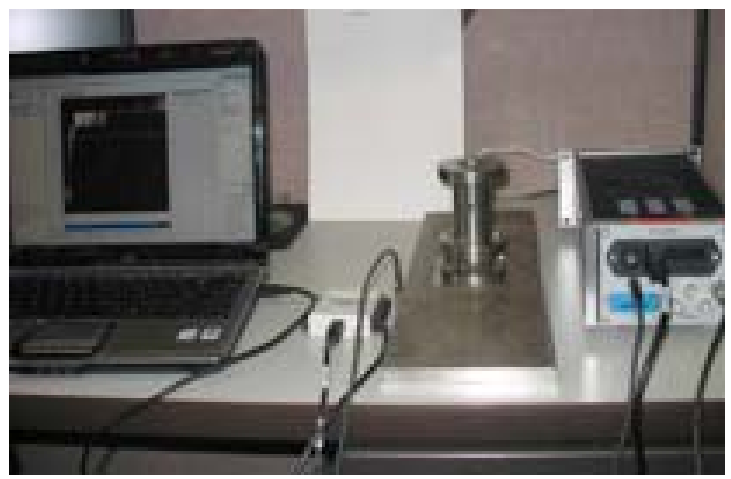

(e)

Figure 2. Sliding plate rheometer. (a) Sliding plate, (b) wall shear stress transducer, (c) disassembled shear stress transducer showing rigid beam, (d) shear stress transducer with capacitance probe, (e) complete assembly with all electrical components

\section{$\underline{\text { Rheological Behavior }}$}

Subsequently, we outline the pertinent shear rheological behavior exhibited by the short glass fiber composite fluids as it will aid in the discussion on model development. When the steady-state rheology of a suspension is compared to its neat counterpart, it typically has an enhanced Newtonian plateau and can exhibit a shear thinning behavior at lower shear rates than the neat resin. At high shear rates the viscosity curves of the composite fluid and its neat suspending medium typically merge. In some cases, typically at very high fiber loading, the suspensions can exhibit yield-like behavior. Point being, the steady-state viscosity can be predicted with a number of shear rate dependent empiricisms, i.e. CarreauYasuda model. Conversely, the transient shear rheology of fiber suspensions is typically easily distinguishable from that of a neat resin. For example, when an undeformed sample is subjected to a start-up of flow experiment, the sample exhibits a relatively large stress overshoot in both the shear stress and the first normal stress difference. This behavior can be seen in Fig. 3 (a) and (b) $\left(\tau^{+}\right.$and $\mathrm{N}_{1}{ }^{+}$ vs. time, respectively) for the $30 \mathrm{wt} \%$ PBT. The relatively large overshoot is believed to be a result of the fibers rotating as a result of the flow field to align in the flow direction. Once the fibers reach a steady-state the stresses reach a steady-state. An interesting observation is that when the flow is removed and then reapplied, after some period of time, the stresses return to their previous value. From this behavior we believe that during stress relaxation the fibers maintain their orientation so that when the flow is turned back on the stresses immediately grow to their previous value. Hence, the transient rheological behavior is coupled with the fiber orientation and being able to model the evolution of orientation is imperative to correctly predicting the rheological behavior and fiber orientation in complex flow simulations. 


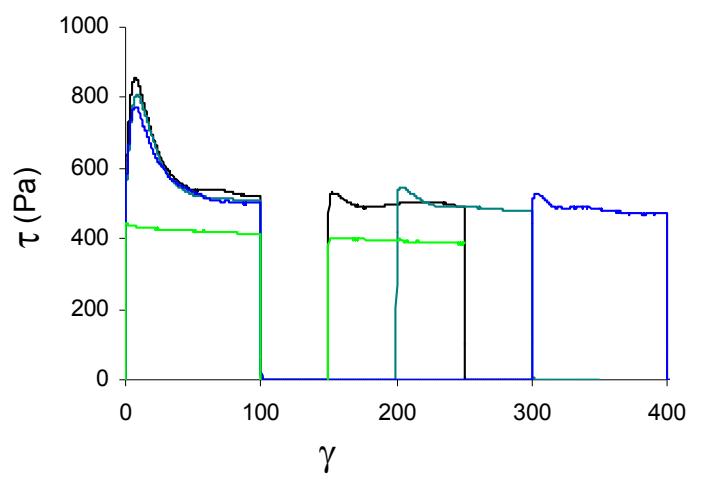

(a)

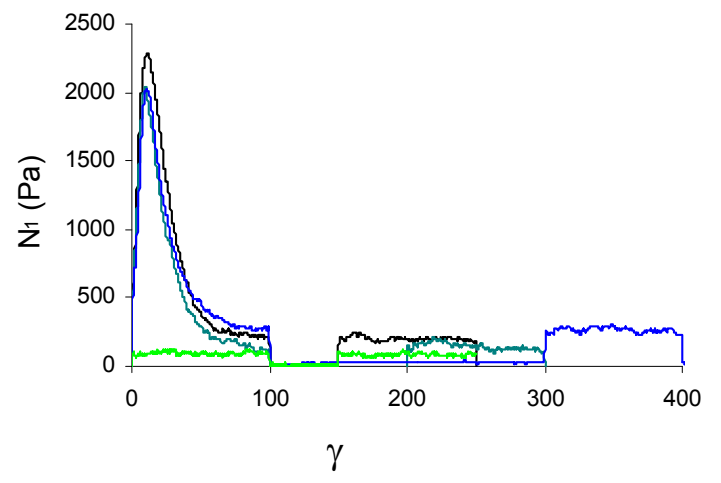

(b)

Figure 3. Stress vs. strain for intermittent stress growth/relaxation tests performed on the $30 \mathrm{wt} \%$ short glass fiber filled PBT. (a) Shear Stress, (b) first normal stress difference. The light green line is the suspending medium. All tests were performed using cone and plate geometry combined.

\section{Theory: Model Development (Short Fibers)}

Evolution of Fiber Orientation: The most common approach to represent the orientation state of a fiber is by defining a unit vector, $\mathbf{u}$, parallel to the fiber backbone. In the case of an ideal suspension which consists of a large number of fibers that are identical in size and shape and whose concentration is spatially uniform the average orientation state of the fibers can be described with an orientation distribution function, $\psi(\mathbf{u}, t)$. However, using the distribution function is numerically cumbersome. Another way to describe the average orientation state of the suspensions is with the orientation order parameter tensor, $\mathbf{A}$, and it is defined as the second moment of the orientation distribution function:

$$
\mathbf{A}(t)=\int \mathbf{u} \mathbf{u} \psi(\mathbf{u}, t) d \mathbf{u}
$$

The trace of $\mathbf{A}$ is always equal to 1 and for a completely random orientation $\mathbf{A}=1 / 3 \mathbf{I}$, where $\mathbf{I}$ is the identity tensor. In the limit where all the fibers are perfectly aligned in the 1-direction the only nonzero component is $A_{11}=1$.

For suspensions containing rigid fibers the most common approach to modeling the evolution of fiber orientation is with the generalized Jeffery equation combined with the Folgar-Tucker term for randomizing fiber orientation. For simple flows the generalized Jeffery equation can be written in terms of $\mathbf{A}$ as follows:

$$
\frac{D \boldsymbol{A}}{D t}=(\boldsymbol{A} \cdot \boldsymbol{W}-\boldsymbol{W} \cdot \boldsymbol{A})+\lambda\left(\boldsymbol{D} \cdot \boldsymbol{A}+\boldsymbol{A} \cdot \boldsymbol{D}-\boldsymbol{D}: \boldsymbol{A}_{4}\right)+2 C_{1}|\boldsymbol{D}|(\boldsymbol{\delta}-3 \boldsymbol{A})
$$

where $\mathbf{W}=\left[(\nabla v)-(\nabla v)^{t}\right] / 2, \mathbf{D}=\left[(\nabla v)+(\nabla v)^{t}\right] / 2, \nabla v$ is the velocity gradient, $\lambda$ is a constant defining the ellipticity of the particle and $\mathrm{C}_{1}$ is a constant defining the magnitude of the randomizing potential. For fibers it is common to assume the particle's aspect ratio approaches infinity and to use $\lambda=1$. Eq. (2) then predicts the period of rotation for the fiber to be infinitely long and is used for all model predictions currently found in literature.

As discussed later (Results and Discussion), the predictions using the generalized Jeffery equation will be compared to our experimental results. From this comparison our research shows that the generalized Jeffery equation over predicts the rate of fiber reorientation. As a result we are unable to 
DOE/NSF February, 2009 Final Report

predict the transient rheological behavior of these composite fluids. It is our hypothesis that this is a direct result of the fibers moving non-affinely with the suspending medium. To account for this behavior we add a term to Eq. (2) referred to as the non-affine motion parameter $\alpha$. This parameter is then implemented into Eq. (2) as follows,

$$
\frac{D A}{D t}=\alpha\left[\boldsymbol{A} \cdot \boldsymbol{W}-\boldsymbol{W} \cdot \boldsymbol{A}+\boldsymbol{D} \cdot \boldsymbol{A}+\boldsymbol{A} \cdot \boldsymbol{D}-\boldsymbol{D}: \boldsymbol{A}_{4}\right]
$$

The non-affine motion parameter effectively retards the rate of fiber reorientation predicted by the generalized Jeffery equation. It's values range between $0-1$ and is thought to be function of fiber concentration, aspect ratio and the velocity gradient. Comparison between experimental data and model predictions for both Eqs. (2) and (3) can be found in the results and discussion section of this report.

Stress contribution: With knowledge of the fiber orientation and deformation gradient the stresses can then be calculated from the following equation:

$$
\sigma=-P \boldsymbol{I}+2 \eta_{s} \boldsymbol{D}+2 \eta_{s} N_{p} \boldsymbol{D}: \boldsymbol{A}_{4}
$$

where $\mathrm{P}$ is the isotropic pressure, $\eta_{s}$ is the suspending medium viscosity, and $N_{p}$ is a dimensionless parameter that describes the magnitude of the total hydrodynamic drag in the system. The terms on the right hand side of the equals sign are the contributions from the isotropic pressure, suspending medium, and hydrodynamic drag of the fluid on the fiber respectively. The suspending medium viscosity, $\eta_{s}$, in Eq. (4) can be taken to be a constant for a Newtonian fluid, a generalized Newtonian fluid in which the viscosity depends on shear rate only and represented with an empiricism such as the Carreau-Yasuda model or predicted using a viscoelastic constitutive equation for fluids that exhibit both viscous and elastic responses. There are various theories for calculating $N_{p}$ for dilute and semi-dilute systems, i.e. Dinh-Armstrong and Shaqufeh-Fredrickson but none for concentrated systems. For our model predictions we fit $N_{p}$ to the peak of the shear stress overshoot. This will be discussed in detail in the results and discussion section.

\section{Theory: Model Development (Long Fibers)}

It is of primary interest to try and extend the existing simulation approach for short glass fiber systems to long glass fiber systems. This has proven to be a difficult task, but we have come up with a possible approach by incorporating what we term a flexibility parameter, $\beta$, into the generalized Jeffery equation as follows:

$$
\frac{D \boldsymbol{A}}{D t}=\alpha\left[\boldsymbol{A} \cdot \boldsymbol{W}-\boldsymbol{W} \cdot \boldsymbol{A}+\boldsymbol{D} \cdot \boldsymbol{A}+\boldsymbol{A} \cdot \boldsymbol{D}-\beta \boldsymbol{D}: \boldsymbol{A}_{4}\right]
$$

In the generalized Jeffery equation the term $\boldsymbol{D}: \boldsymbol{A}_{4}$ is the term associated with stretch along the backbone of the fiber. If we take that same theory but treat the unit vector not as a unit vector along the backbone of the particle, as in Fig. 4 (a), but as a unit vector describing the end-to-end distance and average orientation state of the system, then we can control the amount of fiber flexibility by multiplying the term $\boldsymbol{D}: \boldsymbol{A}_{4}$ by some constant, $\beta$. 


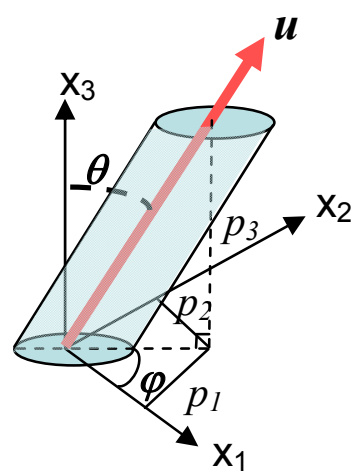

(a)

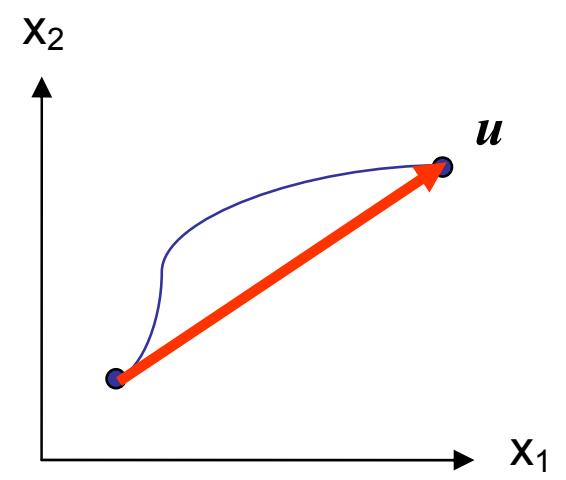

(b)

Figure 4. Unit vector $\mathbf{u}$ : (a) along the backbone of a rigid fiber, (b) an end-to-end vector representing the average position of a bent fiber.

The addition of this parameter $\beta$, has an interesting effect on the transient fiber orientation in startup of flow. This can be seen in Fig. 5 which is the $A_{12}$-component of the tensor A, vs. strain. The magnitude of the shear stress is directly proportional to the magnitude of the $\mathrm{A}_{12}$-component. From this figure one can see that an increase in $\beta$, results in a decrease in the $\mathrm{A}_{12}$ component. Unfortunately, we currently do not have any rheological behavior of a long glass fiber system to compare to at this point(It is the emphasis of the continued research effort).

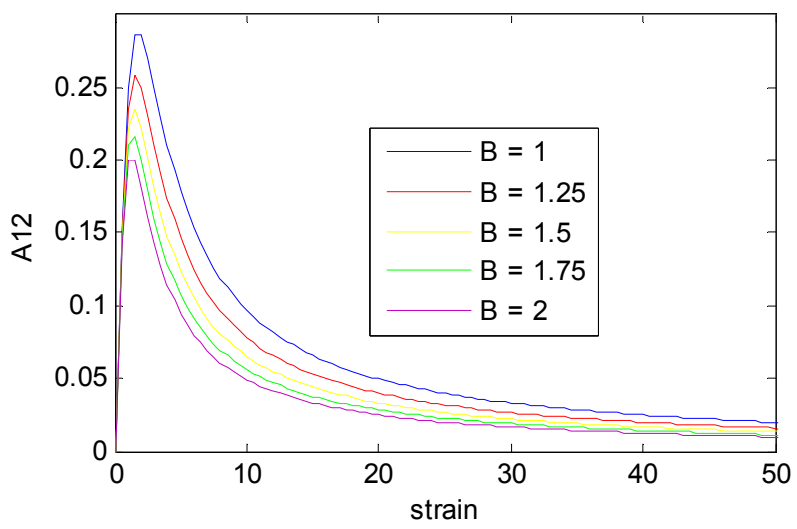

Figure 5. $\mathrm{A}_{12}$ component vs. strain for various $\beta$ values in start-up of flow simulation.

Another approach to modeling long/flexible fiber systems is by simulating the dynamic behavior of individual fibers. This can be done in various ways but the most "straight forward" approach is to begin with the theoretical work of Hinch for an infinitely flexible fiber. In this theoretical development the tension forces within the thread, $T$, are set equal to the viscous forces acting on the thread. As a result two equations are formed, one describing $T$ at discretized points along the thread, Eq. (6) and one defining the time rate of change of the nodal points as a function of $T$ Eq. (7).

$$
\frac{\partial^{2} T}{\partial s^{2}}-\frac{1}{2}\left(\frac{\partial^{2} \vec{x}}{\partial s^{2}}\right) T=-\frac{\partial \vec{x}}{\partial s} \bullet \underline{\underline{D}} \bullet \frac{\partial \vec{x}}{\partial s}
$$


DOE/NSF February, 2009 Final Report

$$
\frac{\partial \vec{x}}{\partial t}=\underline{\underline{\nabla v}} \cdot \vec{x}+\frac{\partial T}{\partial s} \frac{\partial \vec{x}}{\partial s}+\frac{1}{2} T \frac{\partial^{2} \vec{x}}{\partial s^{2}}
$$

In Eqs. (6) and (7), $s$ is the arc length, and $\boldsymbol{x}$ is the position vector. Given an initial fiber orientation one can solve Eqs. (6) and (7) for the time rate of change of the fiber configuration. Model predictions for simple shear flow can be seen in Fig. (6). Strain 0 relates to the initial fiber orientation and strains 1-4 show the evolution of orientation with a localized center of mass. This model predicts that, in simple flow the fiber straightens and aligns itself with the flow direction.

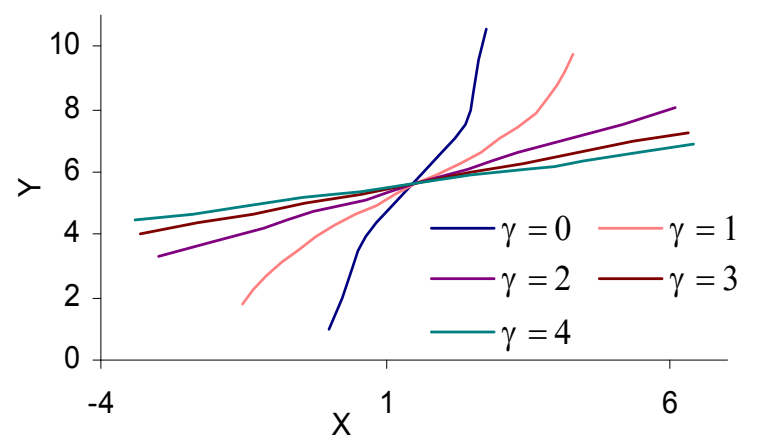

Figure 6. Model predictions for the Hinch infinitely flexible fiber in simple shear flow. Strain 0 relates to the initial orientation and strains 1-4 show the evolution of orientation with a common center of mass.

In addition to Hinch's equation, Goddard and Huang (1983) extended the work for a perfectly flexible fiber by encompassing the concept of mobility into the evolution equation. This model suggests that in concentrated fiber suspensions there exists a preferred direction for the motion of a single fiber. The mobility tensor provides insight into the kinematic restrictions of a fiber caused by the presence of its neighbors. This restricted motion affects the orientation development of the fibers. Therefore, our future work will be to assess this model by studying the mobility tensor in simple shear flow, with the use of the sliding plate rheometer.

$$
\begin{aligned}
& \frac{\partial \vec{x}}{\partial t}-K_{L} \frac{\partial T}{\partial s} \frac{\partial \vec{x}}{\partial s}=T \underline{\underline{K}}_{T} \bullet \frac{\partial^{2} \vec{x}}{\partial^{2}}+\vec{v}(\vec{x}, t) \\
& \frac{\partial}{\partial s}\left(K_{L} \frac{\partial T}{\partial s}\right)-\left(\frac{\partial^{2} \vec{x}}{\partial s^{2}}\right) \bullet\left(K_{L} \underline{\underline{\delta}}+\underline{\underline{K}}_{T}\right) \bullet\left(\frac{\partial^{2} \vec{x}}{\partial s^{2}}\right) T=-\frac{\partial \vec{x}}{\partial s} \bullet \frac{\partial \vec{v}(\vec{x}, t)}{\partial s}
\end{aligned}
$$

Lastly, a relatively recent model was published for semi-flexible fibers in dilute solutions by Strautins and Lats (2007). The authors construct a continuum model that provides a first approximation to flexibility. This is accomplished by modeling a fiber as two rods connected by a pivot allowing bead (Figure 7). 


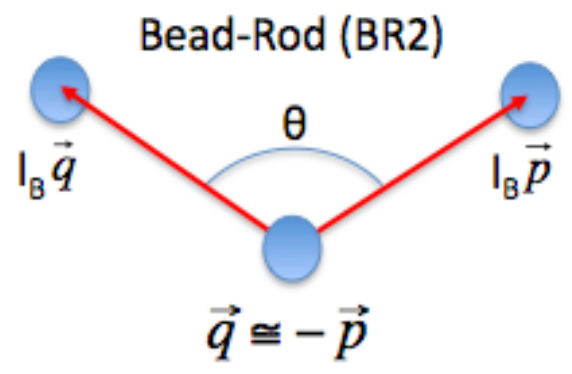

Figure 7: Fiber model, with segment length $l_{\mathrm{B}}$, allowing semi-flexibility. The segment orientations are denoted by unit vectors $\vec{p}$ and $\vec{q}$, and are separated by an angle $\theta$ with some given bending rigidity.

The model considers the flexible fiber to consist of two rigid segments with length $1_{\mathrm{B}}$ that are allowed to slightly pivot about the connecting bead with some restorative bending rigidity, $k$. Both $\vec{p}$ and $\vec{q}$ are unit vectors that represent the orientation of the corresponding fiber segments with respect to the center bead. The model equations are developed and are given as:

$$
\begin{aligned}
& \frac{D \underline{\underline{A}}}{D t}=\underline{\underline{A}} \cdot \underline{\underline{\kappa}}^{T}+\underline{\underline{\kappa}} \cdot \underline{\underline{A}}-\left[\left(\underline{\underline{\kappa}}+\underline{\underline{\kappa}}^{T}\right): \underline{\underline{A}}\right] \underline{\underline{A}}+\frac{l_{B}}{2}[\vec{C} \vec{\mu}+\vec{\mu} \vec{C}-2(\vec{\mu} \cdot \vec{C}) \underline{\underline{A}}]-2 k[\underline{\underline{B}}-\underline{\underline{A}} \operatorname{tr}(\underline{\underline{B}})] \\
& \frac{D \underline{\underline{B}}}{D t}=\underline{\underline{B}} \cdot \underline{\underline{\kappa}}+\underline{\underline{\kappa}} \cdot \underline{\underline{B}}-[(\underline{\underline{\kappa}}+\underline{\underline{\kappa}}): \underline{\underline{A}}] \underline{\underline{B}}+\frac{l_{B}}{2}[\vec{C} \vec{\mu}+\vec{\mu} \vec{C}-2(\vec{\mu} \cdot \vec{C}) \underline{\underline{B}}]-2 k[\underline{\underline{A}}-\underline{\underline{B}} \operatorname{tr}(\underline{\underline{B}})] \\
& \frac{D \vec{C}}{D t}=\underline{\underline{\kappa}} \cdot \vec{C}-(\underline{\underline{A}}: \underline{\underline{\kappa}}) \vec{C}+\frac{\overrightarrow{l_{B}}}{2}[\vec{\mu}-\vec{C}(\vec{\mu} \cdot \vec{C})]-k \vec{C}[1-(\operatorname{tr}(\underline{\underline{B}})] \\
& \vec{\mu}=\sum_{i=1}^{3}\left(\sum_{j=1}^{3} \sum_{k=1}^{3} \frac{\partial^{2} v_{i}}{\partial x_{i} \partial x_{k}} A_{j k}\right) \vec{e}_{i}
\end{aligned}
$$

where the two orientation tensors represent the second moment of the distribution function of the unit vectors, $\vec{p}$ and $\vec{q}$, in the following manner:

$$
\begin{aligned}
& \underline{\underline{A}}=\int \vec{p} \vec{p} \psi(\vec{p}, \vec{q}) d \vec{p} d \vec{q} \\
& \underline{\underline{B}}=\int \vec{p} \vec{q} \psi(\vec{p}, \vec{q}) d \vec{p} d \vec{q}
\end{aligned}
$$

As a direct consequence to the bending rigidity, encompassed within the model parameter $k$, the expectancy of a segment orientation (with respect to the orientation distribution function) may be nonzero in general, and is accounted for in Eqn. (12) by the following definition:

$$
\vec{C}=\int \vec{p} \psi(\vec{p}, \vec{q}) d \vec{p} d \vec{q}
$$

Lastly, Eqn. (13) contributes second order derivatives of the velocity field that originate from a Taylor series approximation applied to the bead kinematics. In simple shear flow, for example, all components are 0 . This vector, in Eqn. (13), is formed by the unit dyads $\vec{e}_{i}$.

Our future work will center on determining how well this model describes long fiber orientation. Currently, the bead-rod model is being explored for the case of a center gated disk, for which preliminary results have been obtained. It is also within our interest to understand what length scales 
DOE/NSF February, 2009 Final Report

this model best describes. It is believed that these efforts will greatly enhance our understanding of long fiber evolution.

\section{Experimental Results vs. Model Predictions}

Subsequently, we compare model predictions of startup of flow to that of experimental results obtained using the $30 \mathrm{wt} \%$ short glass fiber-filled PBT. First we consider predictions using the generalized Jeffery equation, Eq. (2), in combination with Eq. (4) defining the stress contribution. Figure 8 (a) depicts the model predictions for both the shear stress and first normal stress difference vs. strain in which the material parameters $\mathrm{C}_{1}$ (from Bay-Tucker) and $N_{p}$ (from Dinh-Armstrong) are calculated directly from current theory. These predictions are plotted in combination with the experimental rheological behavior. First, the model does predict an overshoot in both the shear and first normal stress difference. However, the model is incapable of predicting the magnitude or the width of either overshoot. It is our hypothesis that being able to predict this transient overshoot behavior is the key to then being able to predict the fiber orientation in the complex flow simulations.

To improve on the model predictions we incorporated the non-affine motion term, Eq. (3), and fit the material parameters instead of calculate them based on theory. $N_{p}$ we fit to the peak of the shear stress overshoot and $\alpha$ we fit to the width of the shear stress overshoot. Model predictions using this approach compared to the experimental results can be seen in Figure 8 (b). Now we come much closure to predicting the transient behavior of both the shear and first normal stress difference.

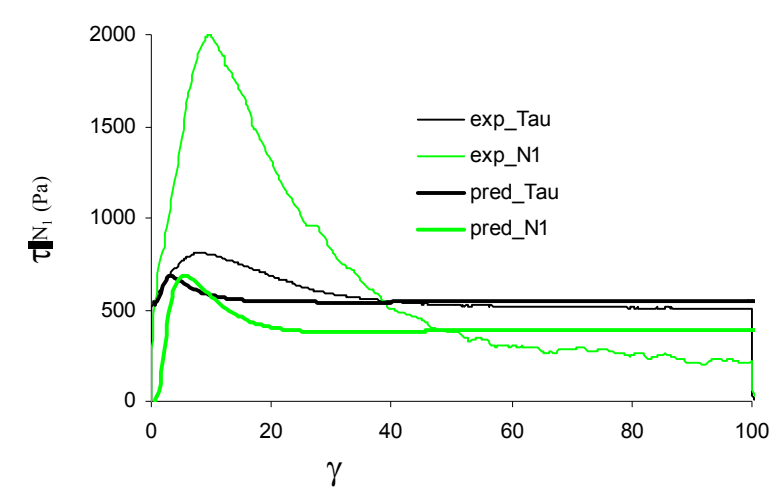

(a)

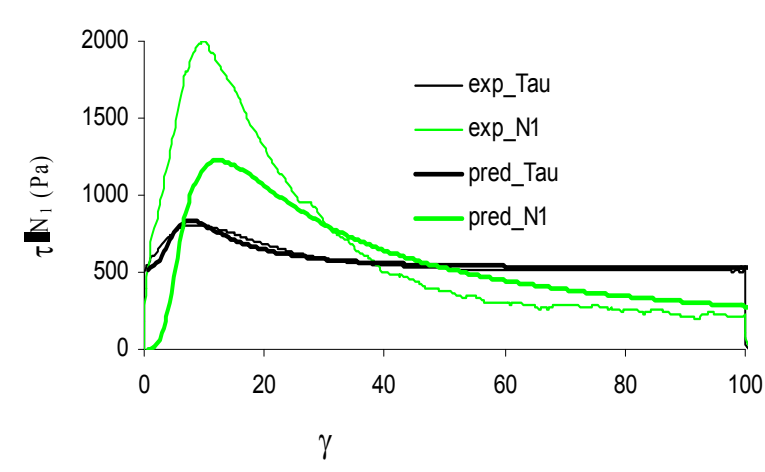

(b)

Figure 8. Experimental results compared to model predictions for the transient stresses in start-up of flow at a shear rate of $1 \mathrm{~s}^{-1}$. (a) Model predictions using current theory to calculate $C_{l}$ and $N_{p}$. (b) Model predictions incorporating non-affine motion and parameters fit to transient shear overshoot. 
The unique model parameters obtained by fitting the transient shear rheology at a shear rate $1 \mathrm{~s}^{-1}$ are also used to predict the behavior at higher shear rates. Figure 9 (a) shows the model predictions, using the same parameters at a shear rate of $4 \mathrm{~s}^{-1}$, and the experimental results for the shear stress and first normal stress difference in start-up of flow. At this shear rate the model actually predicts the transient first normal stress difference better than at $1 \mathrm{~s}^{-1}$. However, the model does not predict the shear stress with as great of accuracy. Figure 9 (b) depicts the peak of the shear and first normal stress difference for the model predictions and experimental results. This figure shows that we can predict both the shear stress and first normal stress difference overshoot using the model parameters found at a shear rate of $1 \mathrm{~s}^{-1}$ over the range of shear rates tested.

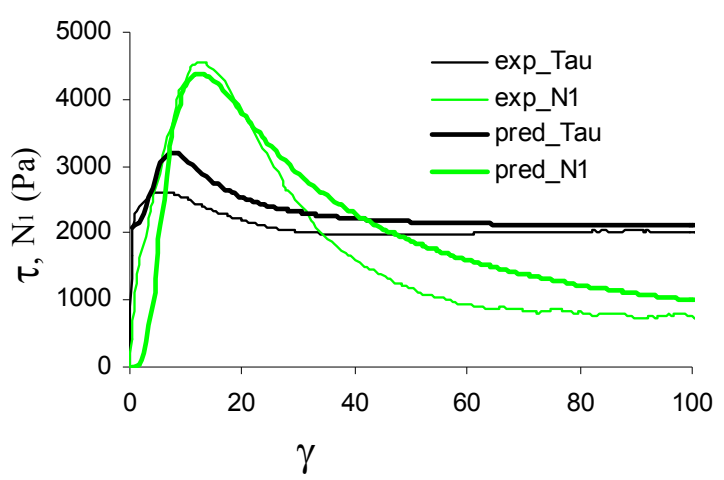

(a)

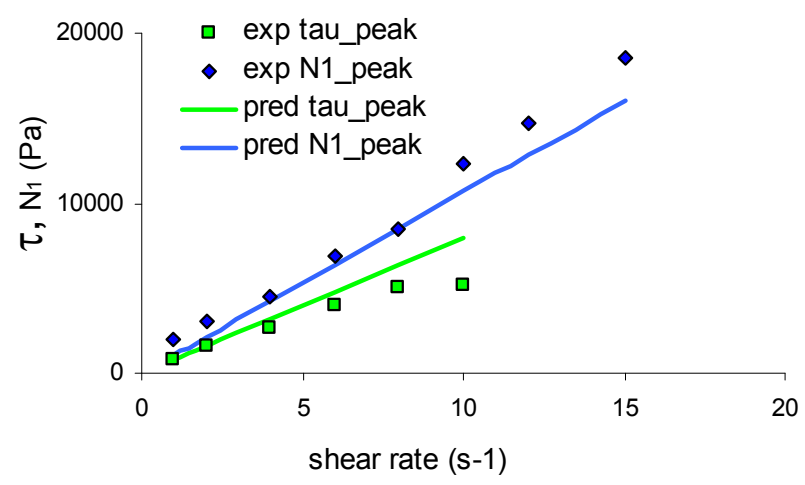

(b)

Figure 9. (a) Experimental results compared to model predictions for the transient stresses in start-up of flow at a shear rate of $4 \mathrm{~s}^{-1}$. (b) Overshoot peak vs. shear rate for experimental results and model predictions. All the model predictions incorporate non-affine motion and parameters fit to transient shear stress overshoot at a shear rate of $1 \mathrm{~s}^{-1}$.

\section{Measurement of Fiber Orientation}

To characterize the evolving fiber orientation under dynamic conditions, donut samples composed of PBT-30 were deformed using the RMS- 800 with the cone and plate geometry at a shear rate of $1 \mathrm{~s}^{-1}$ for a specified amount of time at $260{ }^{\circ} \mathrm{C}$ in a nitrogen environment. Directly after the applied deformation the sample temperature was lowered below the suspension melt temperature. The samples were then bisected at the center of the sample creating a plane perpendicular to the flow direction, encapsulated in epoxy and sanded/polished to a final abrasive particle size of $0.3 \mu \mathrm{m} \mathrm{Al}_{2} \mathrm{O}_{3}$ following standardized techniques. Images of the polished surface were taken using a Zeiss LSM510 confocal laser scanning microscope fitted with a 40x water immersion objective lens and a laser excitation wavelength of $543 \mathrm{~nm}$. The confocal laser is able to focus on the sample surface and penetrate the sample to a depth of $10 \mu \mathrm{m}$. This allows us to construct a 3-D image of the fiber orientation. For each sample ten sequential images were taken from the bottom of the donut sample to the top at a position of $22 \mathrm{~mm}$ from the center. An illustration depicting the position at which the pictures were taken can be seen in Fig. 1. The cross section of each fiber appears as a circle or an ellipse. To process the image, the circumference of each ellipse was traced by hand in power point to improve the contrast between the fibers and the matrix. A simple computer program was written combined with image analysis software in Matlab that measured the position of the center of mass, the major and minor axis and $\theta$ angle of each ellipse. For each sample the total number of ellipses analyzed varied from 321 for strain $=4$ to roughly 456 for strain $=200$. 
DOE/NSF February, 2009 Final Report

We now compare the experimentally determined evolving fiber orientation in startup of flow to that predicted using the generalized Jeffery equation combined with the F-T constant and the non-affine motion parameter. Figure 10 (a) shows experimental results (points) and model predictions using the generalized Jeffery equation (Jeff_ $\mathrm{A}_{\mathrm{ii}}$ ) for the diagonal components of the tensor $\mathbf{A}$. From this figure it is easy to see that the generalized Jeffery equation over predicts the rate of fiber reorientation. We believe this is a result of direct fiber contact. Figure 10 (b) depicts the experimentally determined $\mathrm{A}_{11^{-}}$ component with various model predictions using the generalized Jeffery combined with the calculated F-T constant, Jeff $+F-T$ (calc.)_A $A_{11}$, and the generalized Jeffery combined with slip parameter fit to the transient rheology and the $\bar{F}-\mathrm{T}$ constant fit to the steady state orientation value, Jeff(w/slip)+F$\mathrm{T}$ (fit)_ $\mathrm{A}_{11}$. With the addition of the non-affine motion parameter and by fitting $\mathrm{C}_{1}$ to the steady state orientation we come much closer to predicting the dynamic behavior of the fiber.

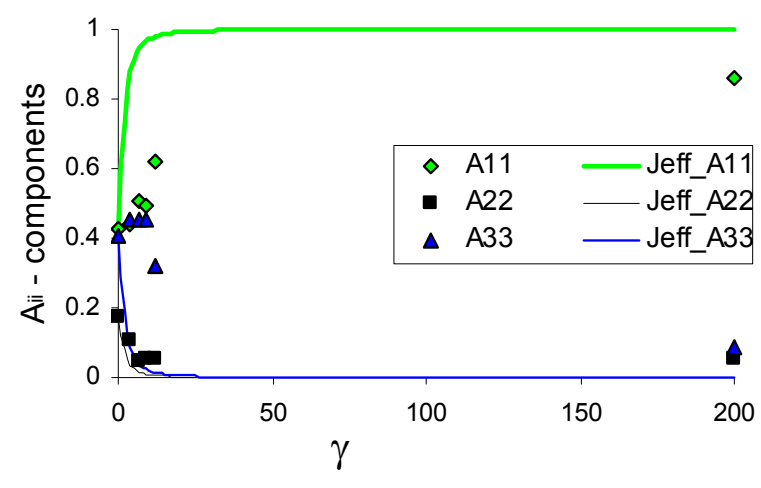

(a)

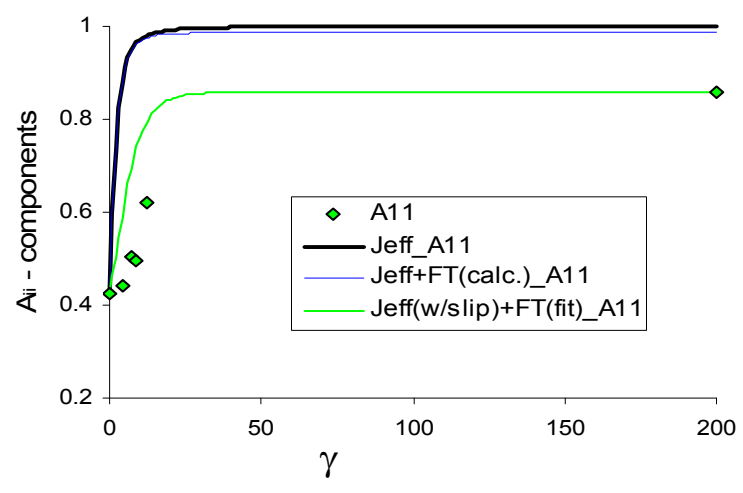

(b)

Figure 10. Experimental results from confocal laser microscopy compared to model predictions for start-up of flow vs. strain at a shear rate of $1 \mathrm{~s}^{-1}$. (a) Experimental results (points) and model predictions using the generalized Jeffery equation (Jeff_ $A_{i i}$ ) for the diagonal components of the tensor $\mathbf{A}$. (b) Experimental results of the $\mathrm{A}_{11}$-component (points), model predictions with the generalized Jeffery equation plus theoretically calculated F-T constant [Jeff $+\mathrm{F}-\mathrm{T}\left(\mathrm{calc}\right.$.) $\left.\mathrm{A}_{11}\right]$, model predictions with the generalized Jeffery equation plus the fit non-affine motion term $\alpha$ F-T constant fit to the steady state orientaiton [Jeff(w/slip)+F-T(fit) $\mathrm{A}_{11}$ ].

\section{Injection Molded Samples/Fiber Orientation Analysis}

The experimental program dealing with the injection molding of parts was finished last year. It consisted of a study including four factors i.e. matrix material, geometry, fiber length, and part thickness of cavity, in 12 combinations. The fiber concentration was chosen to be a constant parameter and selected to be in the concentration range of $30 \mathrm{wt} \%$, typically found in commercially used composites. Figure 11 depicts the factors and combinations involved in the experimental program. The parts were molded to obtain short shots of $75 \%$ of the dimension of the full part. The center-gated disk of $1.3 \mathrm{~mm}$ in thickness, PBT matrix and fibers $500 \mu \mathrm{m}$ has been analyzed. For this part, the short shot size $(\mathrm{R})$ was determined as the difference of the outer minus inner radius.

The evaluation of the orientation was performed using the method of ellipses with images taken from confocal laser microscopy following several publications of Clarke's group. Figure 12 depicts the experimental profile of orientation component $\mathrm{A}_{11}$ along the height of the cavity (z-dir) in the gate 0R, $0.4 \mathrm{R}$, and $0.9 \mathrm{R}$. An asymmetric orientation profile can be seen at the gate region, which corroborates predictions indicated in the literature but never validated e.g. VerWeyst and Tucker, 2002 and Chung and Kwon, 2002. This experimental result is important for assessing our simulations, because the orientation needs to be prescribed at the gate. In the literature the gate orientation has typically been 
DOE/NSF February, 2009 Final Report

assumed constant (random or averaged), especially for a center-gated disk. Figure 12 shows that the fibers aligned in the flow direction close to the walls, far away from the gate. However, $\mathrm{A}_{11}$ profiles are still asymmetric.

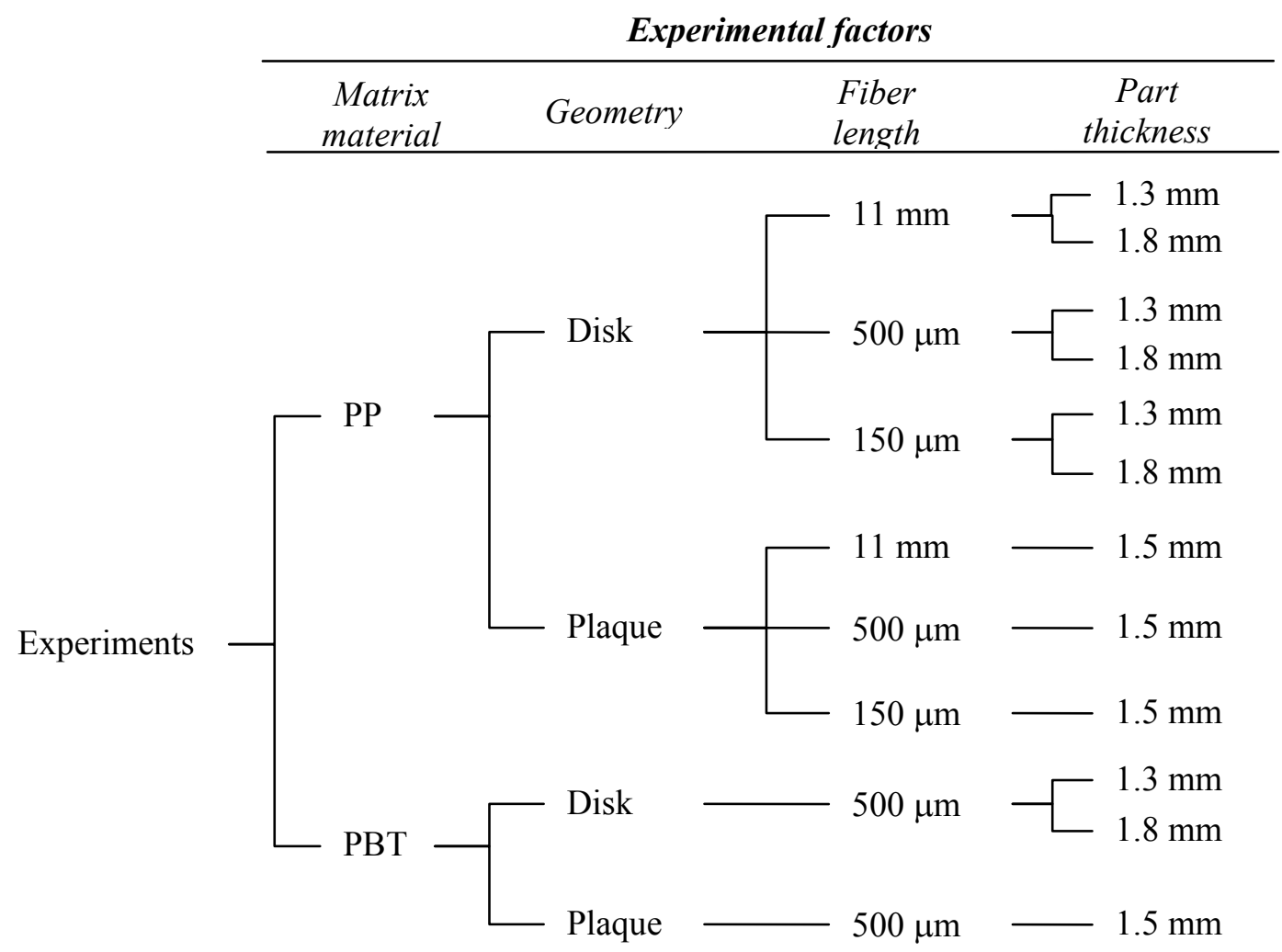

Figure 11 Experimental design.

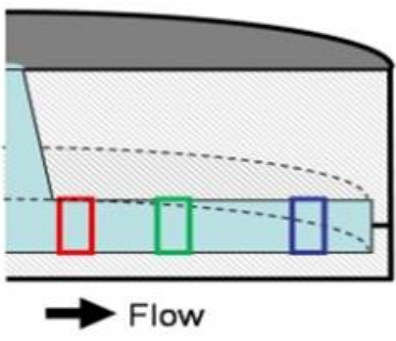

(a)

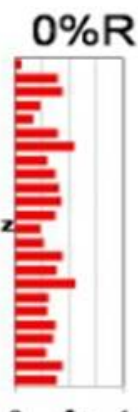

o $\quad A_{11} 1$

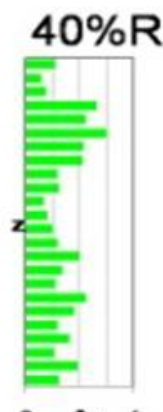

$0 \quad A_{11} 1$

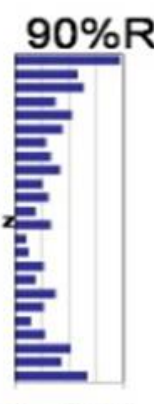

$0 \quad A_{11} 1$

(b)

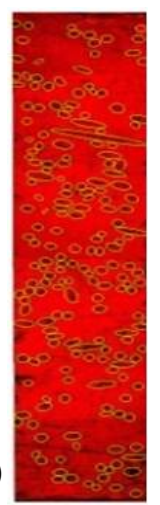

Figure 12 (a)Distribution of orientation component $\mathrm{A}_{11}$ along the gapwise direction in a center gated disk at $0 \% \mathrm{R}, 40 \% \mathrm{R}$, and $90 \% \mathrm{R}$. (b)The typical images obtained by using confocal laser microscopy for the $30 \mathrm{wt} \%$ glass fiber-filled PBT.

The confocal laser microscopy (CLM) technique used to determine the experimental orientation has similar ambiguity problems that are inherent to reflection microscopy techniques for the complex flow experiments. The penetration of the laser beam is limited to $5 \mu \mathrm{m}$ due to the opacity of the matrix. The differences in the ellipses between images at the surface and at $5 \mu \mathrm{m}$ below the surface are only 
DOE/NSF February, 2009 Final Report

perceptible for fibers with a large zenith angle. Therefore, there is an ambiguity for fibers almost perpendicular to the surface plane. A weakness in the CLM technique is the inability to detect fibers parallel to the surface plane inspected. This problem is evident in the regions close to the wall when the $\mathrm{r}, \mathrm{z}$-plane is analyzed. Figure 12 shows this limitation for the positions $0.0 \mathrm{R}$ and $0.4 \mathrm{R}$. An alternative method is under development using the recommendations of Bay and Tucker(1992) consisting of images taken from two mutually perpendicular planes. A medical grade microXCT was used to determine the orientation of fibers below the polished plane, but the resolution of the instrument was not good enough to obtain clear images of the fibers.

A method for orientation and image analysis script has been developed to compute unambiguous values of the orientation tensor. This method consists of plasma etching of a polished plane with the purpose of excavating the surface surrounding the fibers. With this process the fiber borders become exposed, allowing the fiber's unambiguous orientation to be detected and measured with the use of microscopy and digital imaging. Figure 10 (a) shows an optical micrograph containing a polished surface of a fiber with its radial border. It is also worthy to note that this method allows wall bordering fibers to also be accurately measured, Figure 10 (b). This method has the advantages of eliminating the ambiguity problem, let us detect ellipses close to the wall, inspect larger areas of sample, and capture greater number of fibers parallel to the polished plane.

(a)

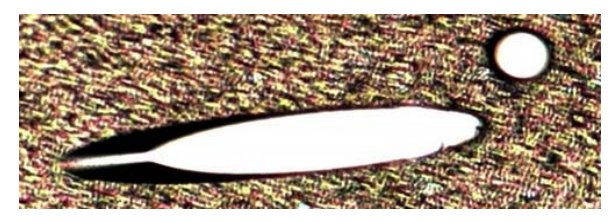

(b)

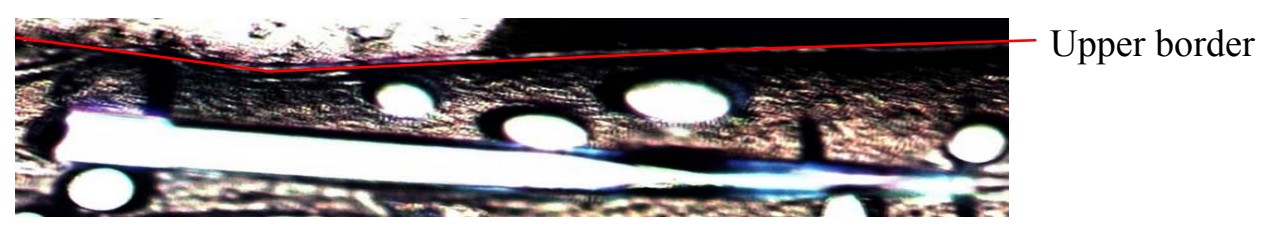

Figure 10 Reflection micrographs for the $30 \mathrm{wt} \%$ glass fiber-filled PP: (a) Surface of polished fiber showing its radial border (b) Group of fibers close to the upper border of a center-gated disk

\section{Simulation}

Short glass fibers: The generalized Jeffery equation containing the non-affine motion parameter, Eq. (5), and the Folgar-Tucker term, last term in Eq. (2), was implemented in the simulation package. In addition various closure approximations have been implemented which include the quadratic, hybrid, linear and IBOF.

\section{Decoupled simulations using delayed generalized Jeffery model.}

Figure 13a shows the measured orientation at the top half of the gate (purple lines) and 0.9R (red lines) for PBT filled with short glass fiber and the prediction at $0.9 \mathrm{R}$ using only Jeffery's model with measured orientation values at the gate (blue line). The prediction using measured gate orientation shows several peaks and valleys that can be related to the layered structure formed at the gate which evolves locally as the flow progresses. The predicted profile at $0.9 \mathrm{R}$ can reproduce, at least qualitatively and in some regions quantitatively, the experimental profile. This is in sharp contrast with predictions using a random orientation at the gate (green line) or a prescribed constant orientation at the gate (not shown) which fail to show the layered structure. These two gate orientations are always used in injection molding simulations and result in similar orientation profiles. All simulations fail to predict the measured orientation for the segment next to the axis of symmetry, the reason for this needs further 
DOE/NSF February, 2009 Final Report

investigation. Results for the orientation component $\mathrm{A}_{11}$ using piecewise gate orientation suggest a fading effect of the initial multilayered structure forming at least a shell-transition-core layer structure at long radial distances from the gate, as illustrated in Figure 13.b. A similar effect has been observed for the other dominant orientation component, $\mathrm{A}_{33}$. The inter-particle interactions through the FolgarTucker term $\left(C_{\Gamma}=0.001\right)$ were considered in the model to try to improve the predictions obtained with the Jeffery model. However, the predictions using the additional terms with experimental piecewise initial orientation produced only slight changes in the predicted orientation, almost overlapping the predictions obtained using the Jeffery's model. Increasing the value of $C_{I}$ to values in the range of 0.016 to 0.003 , commonly found in the literature, e.g. Larson, 1999 and Folgar and Tucker, 1984, also did not significantly change the orientation predictions.

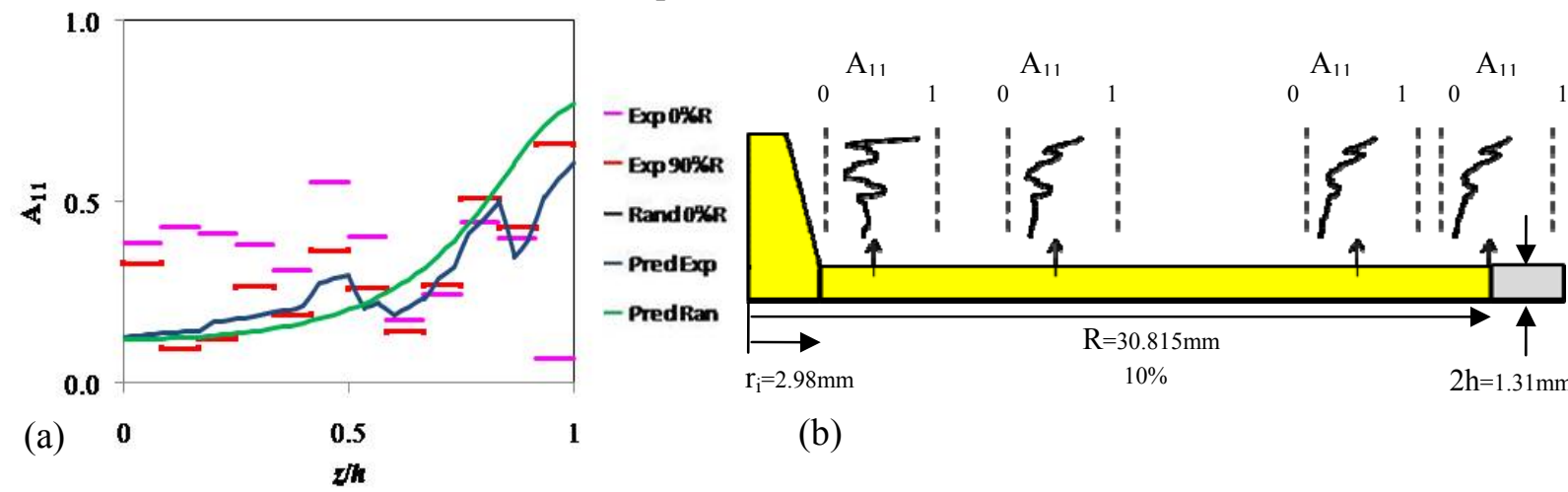

Figure 13 (a) Experimental values of $A_{11}$ at the gate and close to the end of the mold (90\% of radial flow length) with the predicted values of $A_{11}$ orientation component close to the end of fill using random or measured orientation at the gate. (b) Evolution of orientation in a $75 \%$ short shot showing a layered structure expressed through the predicted $\mathrm{A}_{11}$ profiles at several locations in the top half of the disk.

Decoupled simulations using the delayed Folgar-Tucker model.

Figure 14 (a) and (b) shows decoupled simulation results for the radial locations of $40 \%$ and $90 \%$ $\left(R-r_{i}\right)$, respectively, focused on the upper half cavity at a dimensionless simulation time of 290 . These predictions (lines) overestimate the $A_{r r}$ orientation components while they underestimate the $A_{\theta \theta}$ and $\mathrm{A}_{\mathrm{rz}}$ orientation components, when compared to experimental results (symbols). The smooth predicted profile could not reproduce a series of peaks and valleys observed in the experimental profile of orientation. These features in the orientation profile can be related to the layered structure formed at the gate, which evolves locally as the flow progresses as indicated in the previous paragraph. However, when the experimental orientation profile is compared with simulation results at a dimensionless simulation time of 2.90 ( $1 \%$ of the simulation time), the values and features of orientation are successfully reproduced. This implies that the decoupled simulation using the delayed Folgar-Tucker model is evolving faster than the experimental evolution of orientation.

\section{Coupled simulations using delayed Folgar-Tucker model.}

The results of the coupled simulation show improvement in the prediction of the orientation profile. Figure 14 depicts the upper half of the orientation profile at a dimensionless time of 290 for (c) $40 \%$ $\left(R-r_{i}\right)$, and (d) $90 \%\left(R-r_{i}\right)$. The results are sensitive to the use of the delayed Folgar-Tucker model because the retardation of evolution considerably improves the prediction of $\mathrm{A}_{\mathrm{rr}}$, but the profile of the predicted orientation is smooth. In general the $A_{\theta \theta}$ is under estimated, but not as severely as in the decoupled approach. An interesting observation in coupled simulations is that the difference between experimental and predicted $\mathrm{A}_{\theta \theta}$ is greater at $90 \%\left(R-r_{i}\right)$ than at $40 \%\left(R-r_{i}\right)$. This can be an effect of using the Hele-Shaw approximation, because this simplification ignores the existence of the flow front. 
DOE/NSF February, 2009 Final Report

It is well known that the front is a region dominated by extensional flow that is the driving mechanism for $A_{\theta \theta}$. Therefore, these coupled results suggest the need for full simulations, which are especially important in small geometries where the evolution of orientation never reaches the stable structure of orientation.

(a)
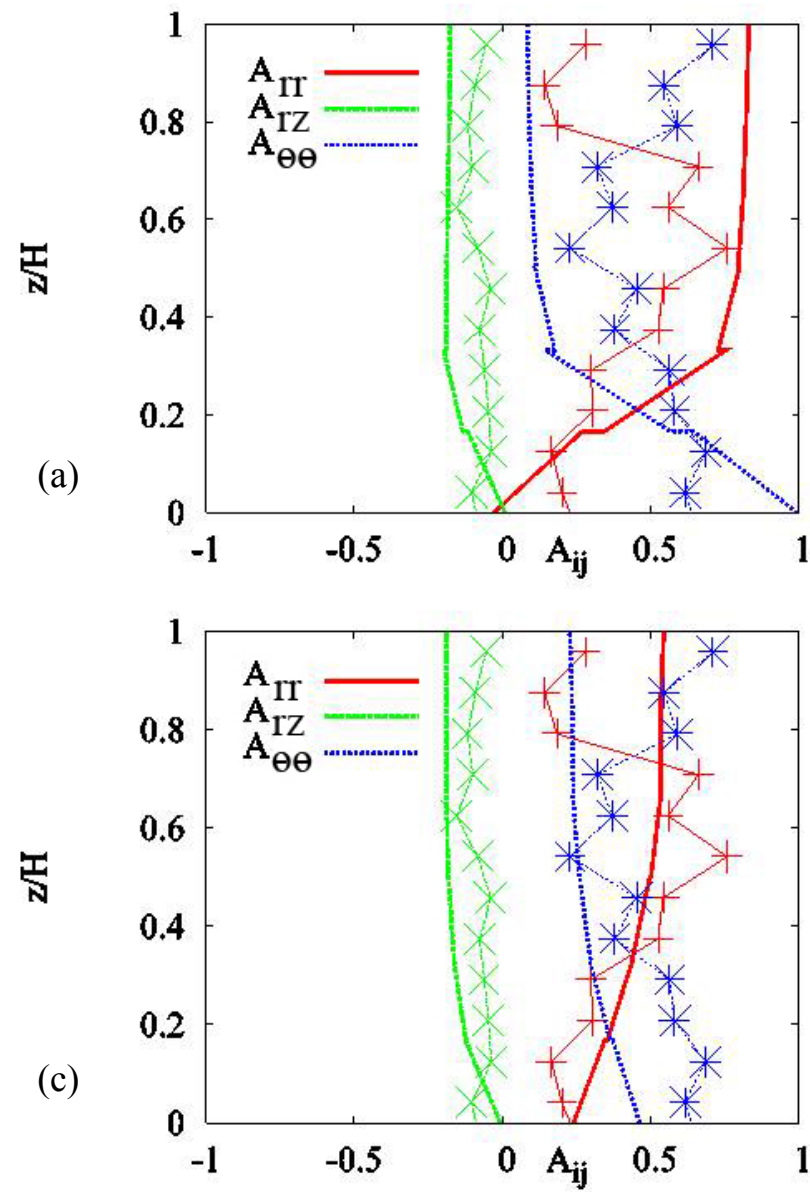

(b)
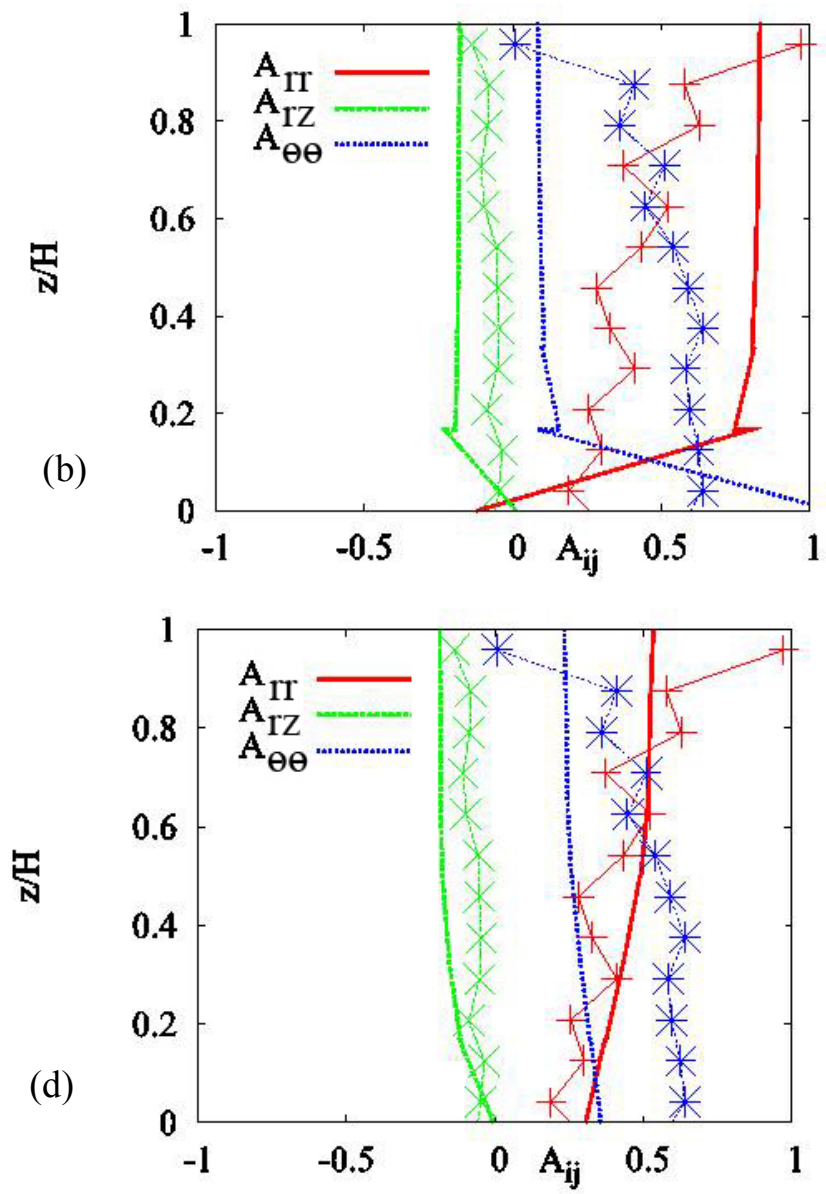

Figure 14 Experimental and predicted orientations from decoupled simulation at (a) $40 \%\left(R-r_{i}\right)$ , and (b) $90 \%\left(R-r_{i}\right)$ and coupled simulation at at (c) $40 \%\left(R-r_{i}\right)$, and (d) $90 \%\left(R-r_{i}\right)$. These simulations were run at a dimensionless time of 290 . The lines denote the predictions, while the symbols are used to indicate the experimental results.

Long glass fibers: The generalized Jeffery equation containing the flexibility parameter, Eq. (5), was implemented in the simulation package. The flexibility parameter accounts for the flexibility of the fibers in a simplified manner. The idea was to evaluate the flexibility as a shrinkage occurring in the end-to-end vector of long glass fibers. To evaluate the model, simulations for short glass fiber PBT composites were performed. Long glass fiber 3-D orientation determination was attempted using a micro X-Ray tomography instrument at Virginia Tech, but the resolution of the instrument was not enough to obtain reliable orientation results. Figure 15 illustrates the experimental and predicted orientation profile for the $A_{11}$ components at $0.4 \mathrm{R}$ (a) and $0.9 \mathrm{R}$ (b) when the flexibility of the fibers is considered. The blue line indicates the predictions for the Jeffery's model $(\beta=1)$ while the green line the predictions for Jeffery's model with flexibility parameter $\beta=0.80$. The results indicate that the 
DOE/NSF February, 2009 Final Report

model does not change significantly in the core region with the addition of the $\beta$ parameter but causes a slight increase in the predictions for $\mathrm{A}_{11}$ close to the wall. In order to predict the orientation in a more robust way, the approach of the bead-rod model in a flow driven system recently introduced by Strautins and Latz was written into the simulation package.

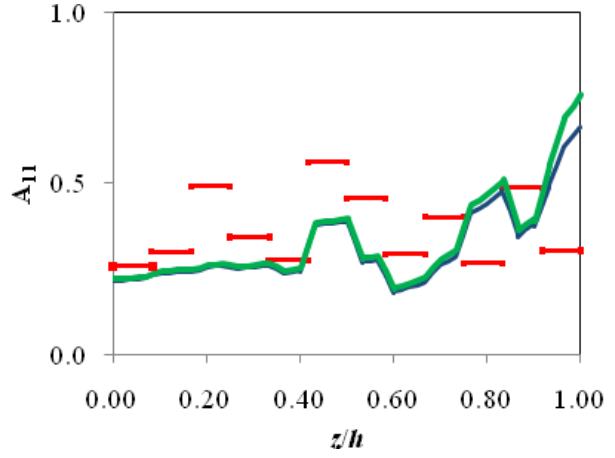

(a)

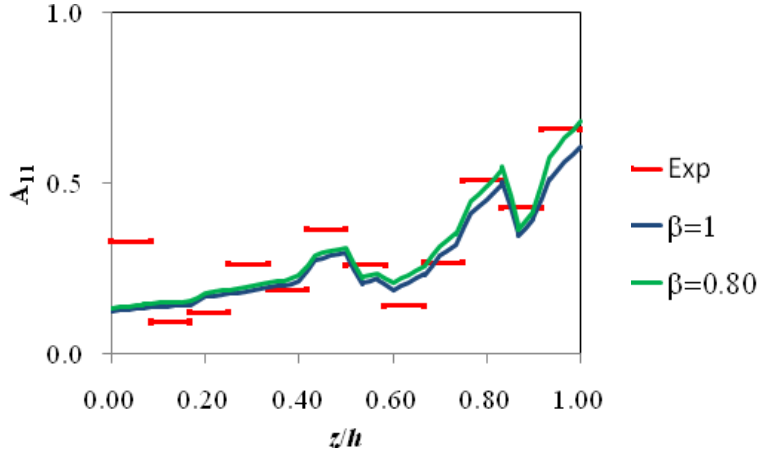

(b)

Figure 15 Predicted and experimental values of $\mathrm{A}_{11}$ at $0.40 \mathrm{R}$ (a) and $90 \mathrm{R} \%$ (b) of a center-gated disk for the Jeffery model with $\beta=1$ and $\beta=0.80$ for the $30 \mathrm{wt} \%$ glass fiber-filled PBT.

In an effort to obtain preliminary results, the bead-rod model was used to simulate the case of a center-gated disk with random initial orientation at the gate. The simulation was performed with a velocity field obtained from parallel disk flow. The simulation results for the fiber orientation were then compared to experimentally determined orientations analyzed at $40 \%$ of the short shot length. Similar to the simulations conducted by the model's author (Strautins and Latz, 2007), the parameter $k$ is presently set equal to a dimensionless value of 0.5. An analysis of this parameter's value will be conducted in the future to ensure its legitimacy. Additionally, the segment length $l_{B}$ was given a dimensionless value that approximately corresponds to $0.5 \mathrm{~mm}$; however, long fiber attrition data is currently being obtained for model length accuracy. The numerical results and experimental data are depicted in Figure 16. For comparison, a simulation using the Folgar-Tucker model (with $\mathrm{CI}=0.01$ ) is also presented in this figure.
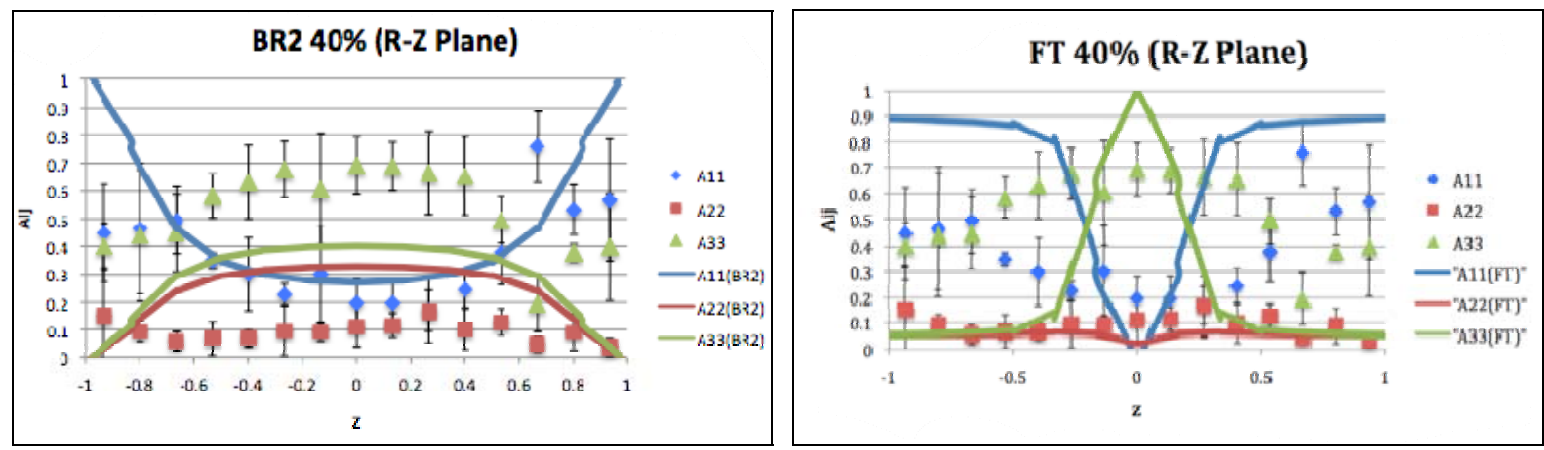

Figure 16: (left) Bead-Rod (BR2) and (right) Folgar-Tucker (FT) simulation and experimentally measured results, for the trace components of the orientation tensor, versus the dimensionless mold thickness at $40 \%$ of the center gated disk length. The components are given by " $r=1 ", " z=2 ", " \theta=3 . "$

Preliminary results for the bead-rod simulation predict a much broader orientation distribution, as compared to what was obtained using the Folgar-Tucker model, which appears to more correctly describe the qualitative shape of the experimentally measured orientations. Additionally, an improvement in the $A_{11}$ component is noticeable, but the $A_{33}$ component is still underpredicted. More 
DOE/NSF February, 2009 Final Report

experimentation, however, is still in need before a complete opinion of this model's validity can be made. Future experiments will focus on fiber length and model parameter effects. Model predictions will then be compared to experiments for validation.

\section{Conclusions}

Short glass fiber suspensions: We have determined a constitutive equation capable of predicting the transient rheological behavior of short glass fiber-filled polymeric fluids. In addition we have developed a method to determine unique material parameters that are independent of shear rate. In general, we have developed a scheme which allows us to obtain the fundamental parameters from simple shear flow and then incorporate these into the simulation package. This is an improvement over the existing approach which using injection molding data(which is what one is trying to simulate). We have also developed a coupled approach in which the velocity field and orientation are calculated simultaneously. The simulations indicate that the orientation measured at the gate is crucial in obtaining the correct orientation pattern inside the cavity(It has been assumed to be random todate).

Long glass fiber suspensions: We have designed and fabricated a sliding plate rheometer primarily for the purpose of performing unbiased and reproducible rheological experiments on long glass fiber-filled polymeric fluids that are otherwise impossible to perform on rotational rheometers. In addition we have developed a simple approach to incorporate fiber flexibility into existing simulation structures for short glass fiber suspensions by incorporating a flexibility parameter $\beta$. Also, the bead-rod model presented within this research was shown to produce broad orientation distributions, a characteristic that preliminary results show to be consistent with long fiber flexibility. The bead rod model is currently being assessed to see if it can correctly explain the dynamics of long fibers in complex flow. Lastly, an extension of Hinch's equation was written for tracking single fibers in concentrated polymer solutions. The use of this model in cooperation with parallel plate experiments will be used to gain further knowledge of long fiber dynamics through the use of mobility. The thrust of the future efforts will be on predicting orientation and configuration of long fibers during molding(compression and injection molding).

\section{Publications as a result of this work(manuscripts, preprints, etc.)}

1. Velez, G. M.; Ortman, K; Eberle, A. P. R.; Wapperom, P; Baird, D. G., "Simulation of Orientation in Injection Molding of a Highly Concentrated, Short Glass Fiber Thermoplastic Composites," SPE ANTEC Technical Paper, accepted on Jan 2009.

2. Ortman, K; Wapperom, P; Baird, D. G., "Long Glass Fiber Orientation in Thermoplastic Composites Using a Model that Accounts for the Flexibility of the Fibers," SPE ANTEC Technical Paper, accepted on Jan 2009.

3. A. P. R. Eberle, D. G. Baird, P. Wapperom, and G. M. Velez-Garcia, "Obtaining Reliable Transient Rheological Data on Concentrated Short Fiber Suspensions Using a Rotational Rheometer", J. Rheol., Accepted.

4. A. P. R. Eberle, G. M. Velez-Garcia, D. G. Baird, and P. Wapperom, "Fiber Orientation Kinetics of a Concentrated Short Glass Fiber Suspension in Startup of Simple Shear Flow", J. Non-Newt. Fl. Mech., submitted.

5. A. P. R. Eberle, D. G. Baird, P. Wapperom, and G. M. Velez-Garcia, "Using Transient Rheology to Determine Material Parameters in Fiber Suspension Theory", J. Rheol., accepted.

6. Velez, G. M.; Ortman, K; Eberle, A. P. R.; Wapperom, P; Baird, D. G., "2-D Simulations of orientation in Highly Concentrated Short Glass Fiber Thermoplastic Composites Made by Injection Molding,"Proceedings of the PPS Americas Regional Meeting, Charleston, South Carolina, 26-29 October 2008. 
DOE/NSF February, 2009 Final Report

7. Ortman, K; Baird, D. G.; Wapperom, P, "Long Glass Fiber Orientation in Thermoplastic Composites Using a Model that Accounts for Flexibility of the Fibers,"Proceedings of the PPS Americas Regional Meeting, Charleston, South Carolina, 26-29 October 2008.

8. Eberle, A. P. R.; Velez, G. M.; Wapperom, P; Baird, D. G., "Fiber Orientation Kinetics of a Concentrated Short Glass Fiber Suspension in Start Up of Shear Flow,"Proceedings of the PPS Americas Regional Meeting, Charleston, South Carolina, 26-29 October 2008.

9. Velez, G. M.; Ortman, K; Eberle, A. P. R.; Wapperom, P; Baird, D. G., "Simulation of Orientation in Injection Molding of High Aspect Ratio Particle Thermoplastic Composites", Proceedings of the XVth International Congress of Rheology, Monterey, California, 3-8 August 2008.

10. Eberle, A. P. R.; Velez, G. M.; Baird, D. G.; Wapperom, P., "The Dynamic Behavior of a Concentrated Non-Brownian Glass Fiber Suspension in Simple Shear Flow", Proceedings of the XVth International Congress of Rheology, Monterey, California, 3-8 August 2008.

11. Eberle, A. P. R.; Baird, D. G.; Wapperom, P., "The rheology of non-Newtonian fluids containing glass fibers: A review of experimental literature," Ind. Eng. Chem. Res., 47:9, 2849-2872(2008)

12. Eberle, A. P. R.; Velez, G. M.; Baird, D. G.; Wapperom, P., "The Dynamic Behavior of a Concentrated Polymeric Suspension Containing Non-Brownian Glass Fibers in Simple Shear Flow," SPE ANTEC Technical Paper, 2008, 868-872.

13. Eberle, A. P. R.; Baird, D. G.; Wapperom, P., "Modeling the rheology and orientation distribution of short glass fibers suspended in polymeric fluids: Simple shear flow," SPE ANTEC Technical Paper, 2007, 65, 2823-2827.

14. Eberle, A. P. R.; Baird, D. G.; Wapperom, P., "Modeling the transient rheology of a polypropylene melt reinforced with long and short glass fibers," SPE ANTEC Technical Paper, 2006, 64, 23152319 .

\section{Presentations directly related to this work}

1. Velez, G. M.; Ortman, K; Eberle, A. P. R.; Wapperom, P; Baird, D. G., "Frontal Flow Effects in 2D Simulations of Orientation in High Aspect ratio Particles Composites Made by Injection Molding," AIChe Annual Meeting, Philadelphia, PA, USA, November 2008.

2. Ortman, K; Velez, G. M.; Eberle, A. P. R.; Baird, D. G.; Wapperom, P, “Long Glass Fiber Orientation in Thermoplastic Composites Using a Model that Accounts for the Flexibility of Fibers," AIChe Annual Meeting, Philadelphia, PA, USA, November 2008.

3. Eberle, A. P. R.; Velez, G. M.; Baird, D.G.; Wapperom, P., "Shear Rheology and Microstructure of a Concentrated Short Glass Fiber-Filled Polybutylene Terephtalate (PBT) ," AIChe Annual Meeting, Philadelphia, PA, USA, November 2008.

4. Velez, G. M.; Eberle, A. P. R.; Ortman, K; Wapperom, P; Baird, D. G., "2-D Simulations of orientation in Highly Concentrated Short Glass Fiber Thermoplastic Composites Made by Injection Molding," PPS Regional Meeting, Charleston, SC, USA, October 2008.

5. Ortman, K; Velez, G. M.; Eberle, A. P. R.; Wapperom, P; Baird, D. G., "Long Glass Fiber Orientation in Thermoplastic Composites Using a Model that Accounts for Flexibility of the Fibers," PPS Regional Meeting, Charleston, SC, USA, October 2008.

6. Eberle, A. P. R.; Baird, D.G.; Wapperom, P.; Velez, G. M., "Fiber Orientation Kinetics of a Concentrated Short Glass Fiber Suspension in Start Up of Shear Flow," PPS Regional Meeting, Charleston, SC, USA, October 2008.

7. Velez, G. M.; Eberle, A. P. R.; Ortman, K; Wapperom, P; Baird, D. G., "Simulation of Orientation in Injection Molding of High Aspect Ratio Particle Thermoplastic Composites," XVth International Congress of Rheology, Monterey, CA, August 2008 
DOE/NSF February, 2009 Final Report

8. Eberle, A. P. R.; Velez, G. M.; Baird, D.G.; Wapperom, P., "The Dynamic Behavior of a Concentrated Non-Brownian Glass Fiber Suspension in Simple Shear Flow," XVth International Congress of Rheology, Monterey, CA, August 2008

9. D.G. Baird, "Rheology and Simulation of Flows of Polymeric Fluids Containing Rigid and Flexible Fibers", University of Naples, June 13, 2008.

10. Eberle, A. P. R.; Velez, G. M.; Baird, D.G.; Wapperom, P., "The Dynamic Behavior of Concentrated Polymeric Suspension Containing Non-Brownian Glass Fibers," SPE ANTEC Annual Technical Conference, Milwaukee, WI, USA, May 2008.

11. Eberle, A. P. R.; Velez, G. M.; Baird, D.G.; Wapperom, P., "The Dynamic Behavior of Concentrated Non-Brownian Glass Fiber Suspensions," Invited lecture: Virginia Tech, Blacksburg, VA, March 2008.

12. Eberle, A. P. R.; Velez, G. M.; Baird, D.G.; Wapperom, P., "The Dynamic Behavior of NonBrownian Glass Fiber Suspensions," Invited lecture: University of Delaware, Newark, DE, January 2008.

13. Eberle, A. P. R.; Velez, G. M.; Baird, D.G.; Wapperom, P., "Rheology and Simulation of Flow of Polymeric Fiber Suspensions," Invited lecture: Florida State University, Tallahassee, FL, November 2007.

14. Eberle, A. P. R.; Baird, D.G.; Wapperom, P., "Experimental Determination of the Relationship Between Fiber Orientation Distribution and Stress Growth in Start-up of Flow for Polymeric Fluids Containing Short Glass Fibers," AIChe Annual Meeting, Salt Lake City, UT, USA, November 2007.

15. Velez, G. M.; Eberle, A. P. R.; Baird, D. G.; Wapperom, P., "Simulation of Highly Concentrated Fiber in a Polymer Melt for a Complex Flow Using Hele-Shaw Approximation," AIChe Annual Meeting, Salt Lake City, UT, USA, November 2007.

16. Eberle, A. P. R.; Baird, D.G.; Wapperom, P., "Experimental Determination of the Relationship Between Fiber Orientation Distribution and Stress Growth in Start-up of Flow for Polymeric Fluids Containing Short Glass Fibers," 79th Annual Meeting of The Society of Rheology, Salt Lake City, UT, October 2007.

17. Eberle, A. P. R.; Velez, G. M.; Baird, D.G.; Wapperom, P., "Rheology and Flow Simulation of Polymer Melts Containing Glass Fibers," European Polymer Congress (EPC) Annual Meeting, Portoroz, Slovenia, July 2007.

18. Wapperom, P.; Baird, D. G.; Velez, G. M.; Eberle, A. P. R., "Numerical Simulation of Polymer Melts Containing Short and Long Fibers," XVth International Workshop on Numerical Methods for non-Newtonian Flows, Rhodes, Greece, June 2007.

19. Eberle, A. P. R.; Baird, D.G.; Wapperom, P., "Modeling the Rheology and Orientation Distribution of Short Glass Fibers Suspended in Polymeric Fluids: Simple Shear Flow," SPE ANTEC Annual Technical Conference, Cincinnati, OH, USA, May 2007.

20. Eberle, A. P. R.; Velez, G. M.; Baird, D.G.; Wapperom, P., "Modeling Polymer Melts Containing Short and Long Fibers: Part I Transient Rheology," AIChE Annual Meeting, San Fransisco, CA, USA, November 2006.

21. Velez, G. M.; Eberle, A. P. R.; Baird, D. G.; Wapperom, P., "Modeling of Polymer Melts Containing Short and Long Glass Fiber. Part II: the Simulation of Injection Molded Parts," AIChE Annual Meeting, San Francisco, CA, USA, November 2006.

22. Eberle, A. P. R.; Velez, G. M.; Baird, D.G.; Wapperom, P., "Modeling Polymer Melts Containing Short and Long Glass Fibers: Part I Transient Rheology," 78th Annual Meeting of The Society of Rheology, Portland, ME, October 2006. 
DOE/NSF February, 2009 Final Report

23. Velez, G. M.; Eberle, A. P. R.; Wapperom, P.; Baird, D. G., "Simulation of Injection Molding Thermoplastics Reinforced with Micro- and Nano-paricles," The Fiber Society Annual Meeting and Technical Conference, Knoxville, TN, October 2006.

24. Eberle, A. P. R.; Velez, G. M.; Baird, D.G.; Wapperom, P., "The rheology of polymers containing macro- and nano-sized particles," Invited Lecture: University of Wisconsin, October 2006.

25. Eberle, A. P. R.; Baird, D.G.; Wapperom, P., "Modeling the Transient Rheology of a Polypropylene Melt Reinforced with Long and Short Glass Fibers," SPE ANTEC Annual Technical Conference, Charlotte, NC, USA, May 2006.

26. Eberle, A. P. R.; Baird, D. G., "Transient Rheology of a Polypropylene Melt Reinforced with Long and Short Glass Fibers," AIChE Annual Meeting, Cincinnati OH, USA, November 2005.

27. Baird, D. G.; Eberle, A. P. R.; Velez, G., "Transient Rheology of a Polypropylene Melt Reinforced with Long and Short Glass Fibers," 77th Annual Meeting of The Society of Rheology, Vancouver, Canada, October 2005.

-Underline denotes presenting author- 
Final Report for Period: 09/2009 - 04/2010

Principal Investigator: Advani, Suresh G.

Organization: University of Delaware

\section{Submitted By:}

Advani, Suresh - Principal Investigator

Title:

Predictive Modeling of Composite Molding Processes: Simulation of Compression-Resin Transfer Molding Process for Manufacturing Net Shape Structures

Senior Personnel

Name: Advani, Suresh

Worked for more than 160 Hours: Yes

Contribution to Project:

Name: Simacek, Pavel

Worked for more than 160 Hours: Yes

Contribution to Project:

Post-doc

\section{Graduate Student}

Name: Bhat, Prabhas

Worked for more than 160 Hours: Yes

Contribution to Project:

Graduate student worked on development of the model

Name: Tang, Wenzhong

Worked for more than 160 Hours: Yes

Contribution to Project:

Undergraduate intern helping with the project

Name: Merotte, Justin

Worked for more than 160 Hours: Yes

Contribution to Project:

Name: Okonkwo, Kenneth

Worked for more than 160 Hours: Yes

Contribution to Project:

graduate research assistant working on experimentally characterizing the permeability of fabric under deformation

\section{Undergraduate Student}

Name: Gebauer, Timo

Worked for more than 160 Hours: Yes

Contribution to Project:

Worked on Experiments required in this project
Submitted on: 06/02/2010

Award ID: 0521789

\section{$\underline{\text { Project Participants }}$ \\ Project Participants}




\title{
Other Participant
}

\section{Research Experience for Undergraduates}

\section{DEPARTMENT OF ENERGY}

\section{Organizational Partners}

They provided preforms to measure permeability of the $\mathrm{P} 4$ fabric

Bob Norris from Oakridge national lab

\section{Other Collaborators or Contacts}

\section{Activities and Findings}

\begin{abstract}
Research and Education Activities: (See PDF version submitted by PI at the end of the report)
Compression Resin Transfer Molding (CRTM) Process was investigated in laboratory settings, using specialized setup. Using Continuum Mechanics, a model was developed and Non-Dimensional Analysis was carried out to determine important process parameters.
\end{abstract}

Modifications needed to model CRTM with LIMS (RTM Modeling Package) were made and tested. Material properties needed for modeling CRTM were measured.Experimental Method to visualize the flow in CRTM was developed. Comparisons between experimental results and model predictions were made.

On the educational side, the material characterization technique was introduced in course on Introduction to Principles of Composites Manufacturing (MEEG 655). The details of the approach and the results are attached in an attached word file.

The comparison of experimental results with the existing modeling capability exhibited discrepancies. The issue has been analyzed and it has been traced down to one of the assumptions made in the model. This assumption that the fiber preform does not deform before physical contact with movable mold has been proven to be highly inaccurate, even for stiff preform fiber materials such as the P4 fabric. The experiments clearly show that the preform does deform significantly due to the fluid pressure even if the mold has not made contact.

A modified model in which this assumption is relaxed was developed. One dimensional model of the process that includes the preform deformation throughout the entire process (resin injection, gap closure, preform compaction) has been created and successfully compared to additional experiments.

A new two dimensional flow model with full coupling with fabric compaction was developed and numerically coded to create a simulation. Also a second generation flow validation of CRTM process setup was designed, fabricated and instrumented to monitor the flow during CRTM process.

New experimental setup and tooling were developed to provide:

1.Better experimental instrumentation.

2.The experimental capability to investigate the flow in composites with different length to width ratios, particularly in elongated, large aspect ratio structures.

3.Provide constant compaction force throughout the entire process.

\section{Findings: (See PDF version submitted by PI at the end of the report)}

It was shown that CRTM process can be described similarly to other LCM processes and that numerical modeling within an existing RTM simulation was possible by modifying the script of the RTM package.

It was shown that existing LCM modeling can be applied to CRTM.

with some restrictions. Non-dimensional analysis showed that the number of parameters that influence the process was very limited. 
It was found that we need to account for fabric deformation even during phase I and II as our experiments clearly show this phenomenon. Hence a new modified model was developed to account for this change. A file is attached with a summary of the activities and the findings.

\section{Training and Development:}

Numerical modeling

Experimental Design

Material Characterization

Development of flow visualization method

process model validation

\section{Outreach Activities:}

Introduced the process to many industries that visit Center for Composite Materials and also in my class on Priniciples of Composites Manufacturing that is offered locally and through out reach across the country

\section{Journal Publications}

Simacek, P; Advani, SG; Iobst, SA, "Modeling Flow in Compression Resin Transfer Molding for Manufacturing of Complex Lightweight High-Performance Automotive Parts", JOURNAL OF COMPOSITE MATERIALS, p. 2523, vol. 42, (2008). Published, $10.1177 / 002199830809632$

Bhat, Prabhas; Merotte, Justin; Simacek, Pavel; Advani, Suresh G.,, "Process analysis of compression resin transfer molding,", Composites Part A: Applied Science and Manufacturing, p. 431, vol. 40, (2009). Published,

Merotte, Justin; Simacek, Pavel; Advani, Suresh G.,, "Flow analysis during compression of partially impregnated fiber preform under controlled force", Composites Science and Technology, p. 725, vol. 70, (2010). Published,

Merotte, Justin; Simacek, Pavel; Advani, Suresh G., "Resin flow analysis with fiber preform deformation in through thickness direction during Compression Resin Transfer Molding", Composites Part A: Applied Science and Manufacturing, p. 881, vol. 41, (2010). Published,

\section{Books or Other One-time Publications}

\section{Web/Internet Site}

\section{Other Specific Products}

\section{Contributions within Discipline:}

\section{Contributions}

\section{Contributions to Other Disciplines:}

Contributions to Human Resource Development:

Contributions to Resources for Research and Education:

Contributions Beyond Science and Engineering: 


\section{Categories for which nothing is reported:}

Any Book

Any Web/Internet Site

Any Product

Contributions: To Any within Discipline

Contributions: To Any Other Disciplines

Contributions: To Any Human Resource Development

Contributions: To Any Resources for Research and Education

Contributions: To Any Beyond Science and Engineering

Any Conference 


\section{Modeling and Simulation of Compression Resin Transfer Molding}

Principal Investigator: Suresh G. Advani

Co-Pi: Pavel Simacek

Department of Mechanical Engineering and Center for Composite Materials

University of Delaware

Newark, DE 19716

(302) 831-8975 ;fax: (302)-831-8525; e-mail: advani@me.udel.edu

Contractor: University of Delaware

Contract No.: DMI-0521789

\section{Introduction}

Compression Resin Transfer Molding (CRTM) process belongs to Liquid Composite Molding (LCM) Processing Family. In these processes, the stationary reinforcement preform is placed in mold and injected with liquid resin. Once the preform is fully impregnated, resin is cured and the resulting composite part can be demolded. The resin should cover all empty spaces between fibers so that the composite performance is not compromised.

In CRTM, there is initially a gap left between the preform and the top mold surface to evenly distribute the resin during the injection. Once the required amount of resin is injected into the mold, the gate is closed and the upper mold starts compacting the resin and the preform, squeezing the resin out of gap into the yet unsaturated region in the preform and compacting the preform to the desired fiber volume fraction.

CRTM process has the potential to provide net-shape parts with high quality surface finish with a fast process cycle, not possible with other LCM variations.

\section{Objectives}

The objective of this project is to understand, describe and model the resin flow and preform deformation during the Compression Resin Transfer Molding (CRTM) process. The resulting flow description is used to accurately simulate the resin filling. This will enable identification of possible processing issues, to ensure part integrity and to optimize the resin injection.

\section{Previous Accomplishments}

Mathematical description of the flow during CRTM injection was derived and important parameters and constitutive relations were identified. Material characterization methods were developed to characterize the necessary relations between compacting load, material fiber volume fraction and permeability. Simple experimental setup was developed to examine the process under laboratory conditions.

Using several assumptions, Our LCM flow modeling package was adopted to model CRTM process driven by either applied displacement or constant force. Nondimensional analysis was performed on the governing equations, significantly reducing the number of independent parameters that influence the process. 


\section{Activities in 2008-2009}

- The non-dimensional analysis of the governing equations was continued and the resulting influential parameters were identified. This work was submitted and is accepted for publication in Composites Part A: Applied Science and Manufacturing

- Experimentally established material constitutive relations from previous work were approximated and embedded in the current numerical models.

- The numerical model used previously was used to simulate CRTM experiments performed in the laboratory. The comparison of results uncovered some discrepancies.

- Additional experiments were conducted to find the source of the differences. It was found that, contrary to assumptions, preform significantly deforms during the second stage, when the mold is closing but the preform and mold are still separated by the resin in gap.

- Simple, one dimensional model was developed to simulate the second stage of the CRTM injection allowing for preform deformation. This model assumes that the resin flows only through the thickness of the preform. The model was compared to the experimental results.

- A parametric study was performed to estimate the effects of material parameters on flow during the second stage.

\section{Principal Findings}

- The non-dimensional analysis of the problem proved successful. It reduces the number of significant parameters to five, some of which are insignificant over usual range of processing parameter. Using these (published) results one can estimate the process time/pressure for given material and process parameters.

- Generally accepted model assumption is that the preform does not deform before it comes into contact with moving mold and then it deforms uniformly through the thickness. This assumption proved inaccurate, even for stiff materials like the P4 preform. The experimental data show significant preform deformation before the contact happens, on the order of $10 \%$ for P4 material.

- The comparison of the numerical model with the experimental results reflects this inaccuracy.

- The preform deformation during the second stage can be incorporated into the model which improved the correlation with the experimental data. However, this increased the complexity of the model.

- Simple, one dimensional model that allows preform to deform non-uniformly before the contact with mold was successfully tested and compared to experimental data.

\section{Future Work}

- Develop the 2D simulation tool for the complete process that allows non-uniform preform deformation without contact with the tool.

- Test and validate the numerical results by comparison with the experimental data. 


\section{CRTM Process}

All Liquid Composite Molding (LCM) processes first place a fibrous preform in a mold, which is consequently sealed. Then, a liquid resin is injected into the mold through a gate to saturate the preform. Once all the empty spaces between the fibers are filled with resin, the resin is allowed to cure. During the curing process, the resin cross-links and hardens, the mold is opened and the part is removed.

To address the low production rate of usual (RTM, VARTM) processes, a novel variation of LCM, Compression Resin Transfer Molding (CRTM) is being explored for high volume production. This process (Figure 1) combines resin injection into the preform in a partially open mold subsequently closing the mold to squeeze the resin into the preform and simultaneously compressing the preform to increase the fiber volume content, which is necessary for structural components. CRTM offers the potential to manufacture moderately sized structures in less than a minute or two while preserving the advantages of LCM and net-shape manufacturing with good surface finish.

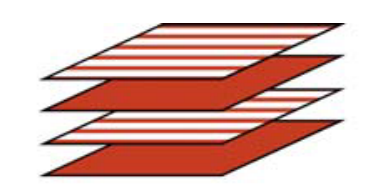

1. Preform Manufacturing

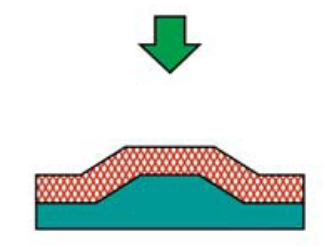

2. Lay-up and Draping

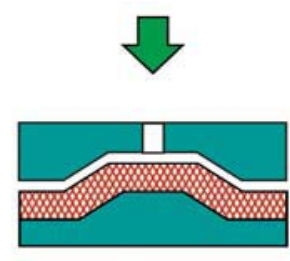

3. Partial Mold Closure

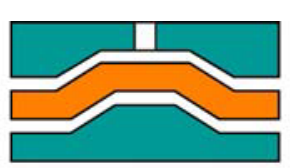

6. Cure, Demolding and Final Processing

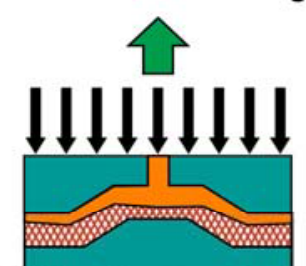

5. Gap Closing: Resin Forced to Saturate Preform
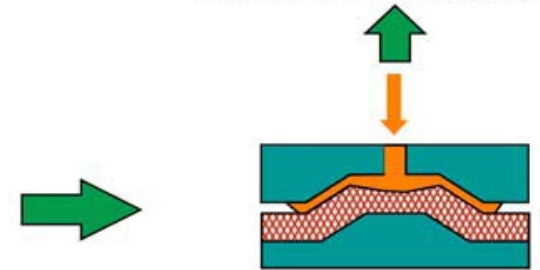

4. Resin Injection into Gap

Figure 1: Schematic of the CRTM process.

The most significant issues encountered during production are (1) dry preform regions caused due to resin not being able to fill all the empty regions between the fibers, resulting in unacceptable parts and (2) low yield and poor repeatability and reliability of the process due to lack of predictive methods to address anomalies in the flow which can help in making the process robust to handle some uncertainties in fabric architecture during manufacturing. As it impractical to apply trial-and-error approach to each part 
manufactured by this process, a process model and simulation is created to describe the resin flow behavior and its coupling with preform compaction during the CRTM process.

\section{Process Model}

\section{Simulation of CRTM using existing tools}

\section{Utilization of LIMS (Liquid Injection Molding Simulation) Package}

We have extended the existing simulation package for liquid composite molding (LIMS) to simulate the Compression Resin Transfer Molding (CRTM) process along the following lines (Figure 2).

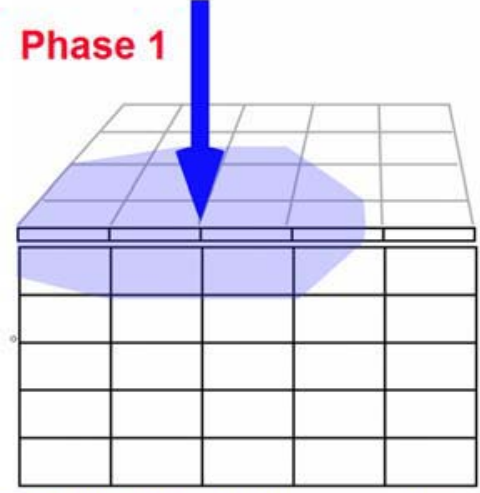

2D GAP: Fixed with permeability obtained from lubrication theory. 3D PREFORM: Fixed porous media.

RESIN SOURCE: Conventional inlet like in RTM.
Phase 2

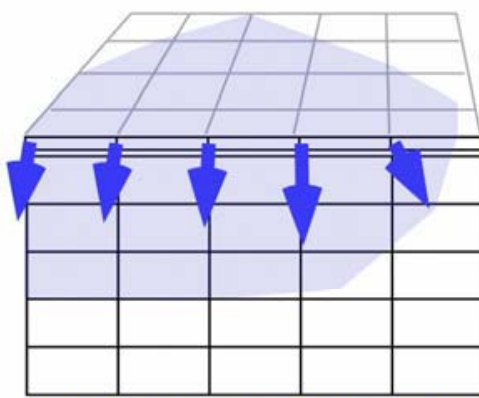

2D GAP: Thickness and permeability varies. 3D PREFORM: Fixed porous media.

RESIN SOURCE: In all filled gap nodes.
Phase 3

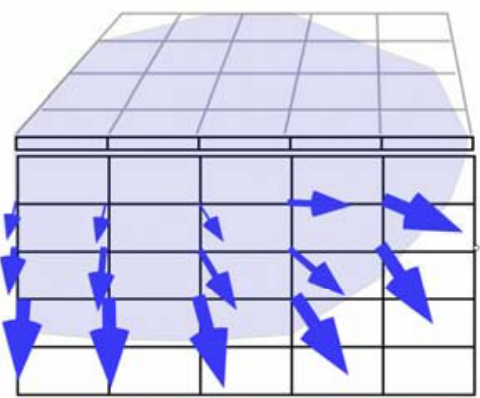

2D GAP: Eliminated by 0 thickness. 3D PREFORM: Fiber volume fraction and permeability varies.

RESIN SOURCE in all filled nodes.

Figure 2. Three phases of CRTM process modeling using LIMS package.

1. In the first stage, the resin is being injected into the gap between the preform and mold. This is simulated like ordinary RTM injection into a porous media with a fixed value of permeability.

2. The mold platen moves closing the gap. The resin is pressed into the preform. LIMS model describes the infusion using area source over the part surface, with the magnitude corresponding to the deformation rate. The preform is assumed to be non-deforming in this stage.

3. The mold contacts the preform and starts compacting the preform. This squeezes the resin out of the filled domain and saturates the dry areas in the preform. The preform deformation is assumed to be uniform through the thickness, the squeeze flow is simulated by source distributed throughout the filled volume. 
This approach was applied to CRTM infusion of both laboratory tests and real parts. The comparison with short shots ([1], Figure 3) was reasonable, but the laboratory experiments demonstrated limits of the validity of our assumptions.
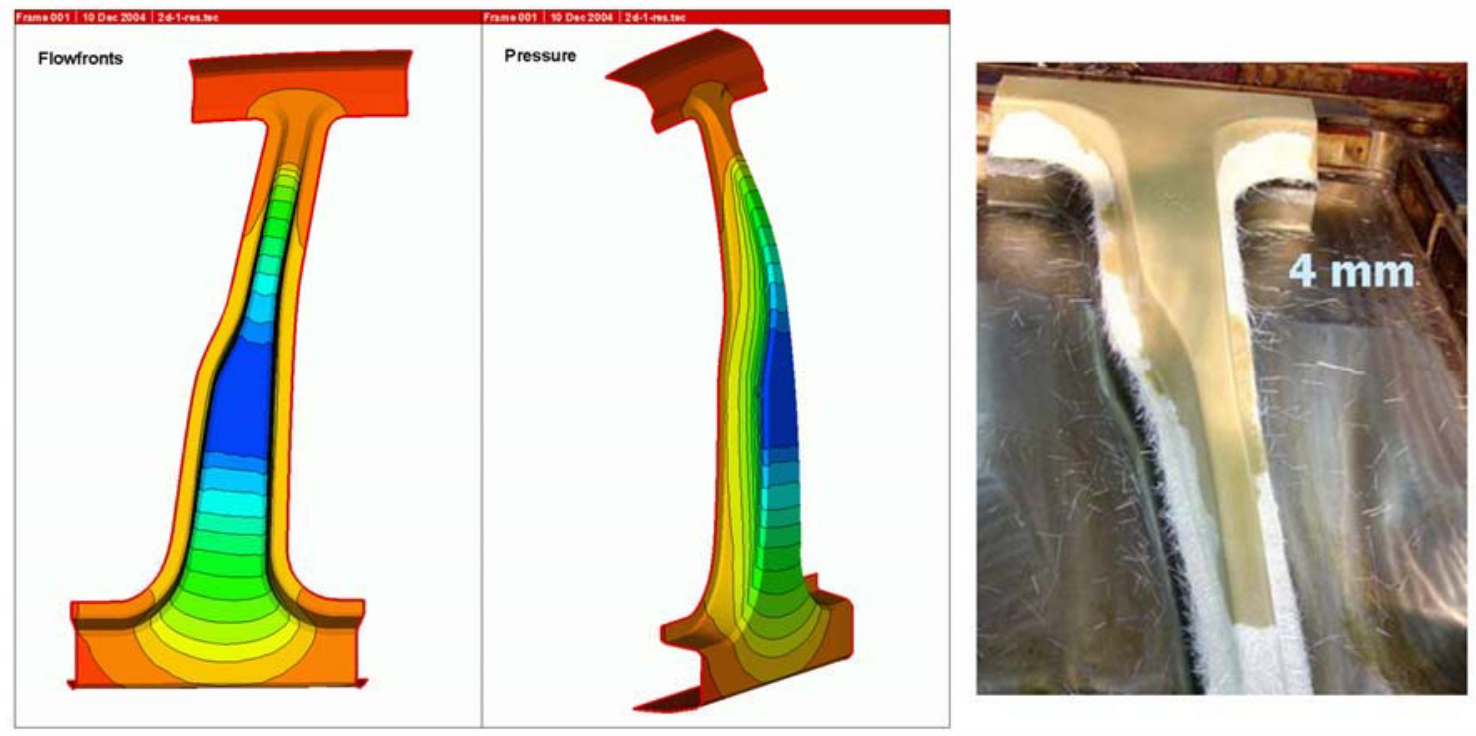

Figure 3. Simulation of CRTM infusion into B-pillar and the short-shot infusion results.

The issues in the third stage concerned the mass conservation and proper scaling of the parameters for the compacting preform. This was addressed by further modification of the LIMS model, allowing actual deformation of the mesh during the third stage ([2], Figure 4). With this approach, the simulation of CRTM infusion is currently possible.

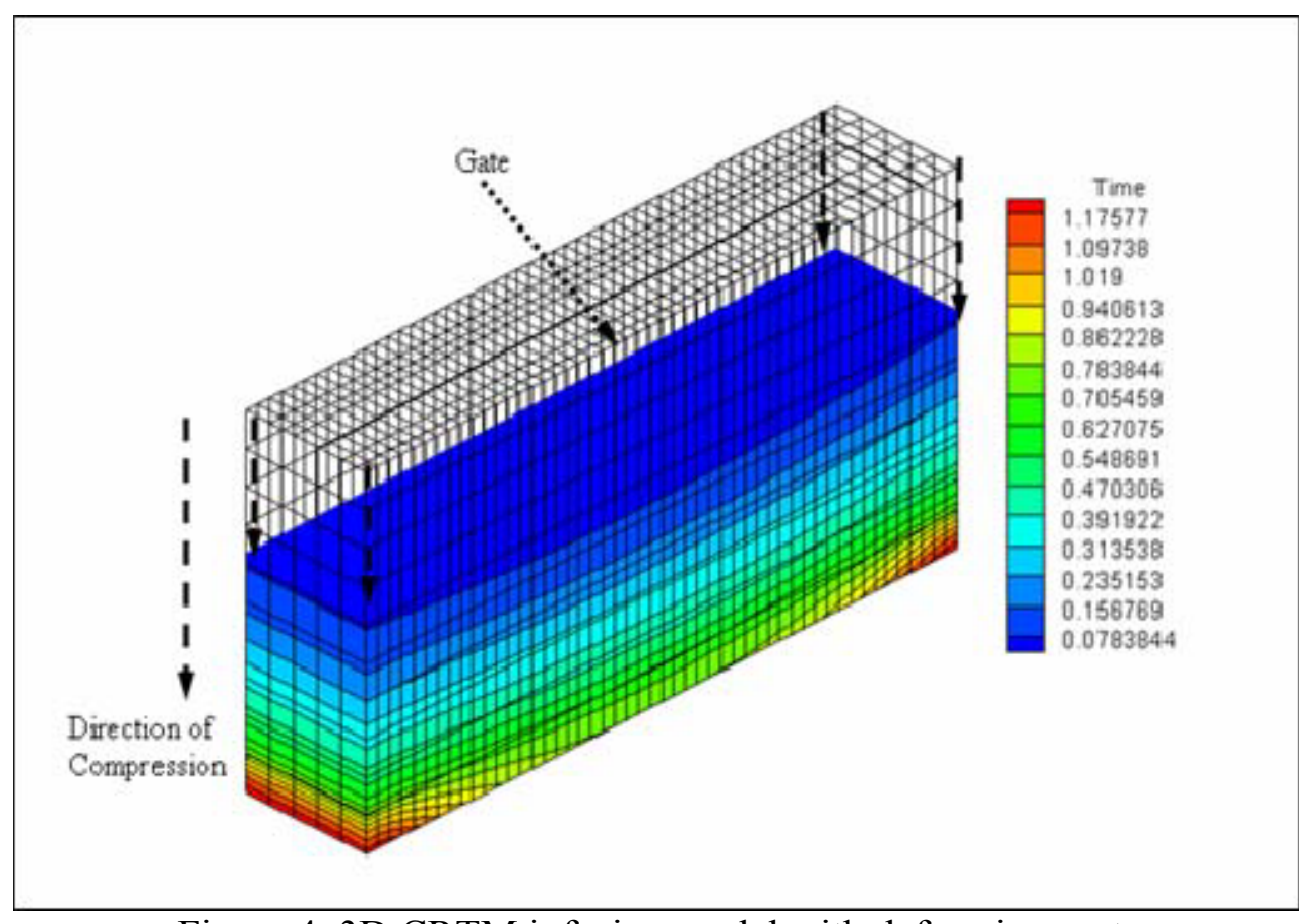

Figure 4. 3D CRTM infusion model with deforming part. 


\section{Analytic Tools for Special Cases}

In the final stage of phase 3 of CRTM process, the resin displaced by preform compaction must fill the remote extremities of the part in virtually in-plane flow. To estimate the times and or pressure requirements for this stage, which is computationally demanding, one can use simple approximate expression ([1]) or numerical approach ([3]).

The latter model was experimentally verified. Using the numerical tools developed in [3], the influence of individual processing parameters during the third stage was determined. It was demonstrated that while the intuitive conclusion that higher applied force equals higher process rate is correct, the actual process speed-up improvements are negligible for very high forces. This implies existence of an optimum driving force that balances the process rate with the process cost.

\section{Simulation of CRTM with Coupling Between Deformation and Pressure}

To model the preform deformation during the second (and first, as needed) phase of CRTM infusion, the governing equations must couple the flow, resin pressure, and the deformation within the preform. This becomes an obstacle because the existing LCM simulation framework of LIMS, cannot be applied any more.

\section{Experimental Observations vs Model Assumptions}

The experiments, described below, revealed a shortcoming of the second phase assumption that the preform does not deform during the second phase of the process. The varying positions of the resin-mold and the resin-preform boundaries are shown in Figure 5. To capture this effect, the preform deformation must be introduced into the model during the second phase. 

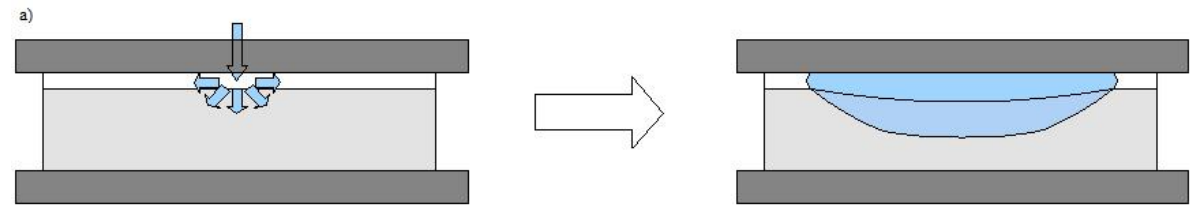

b)
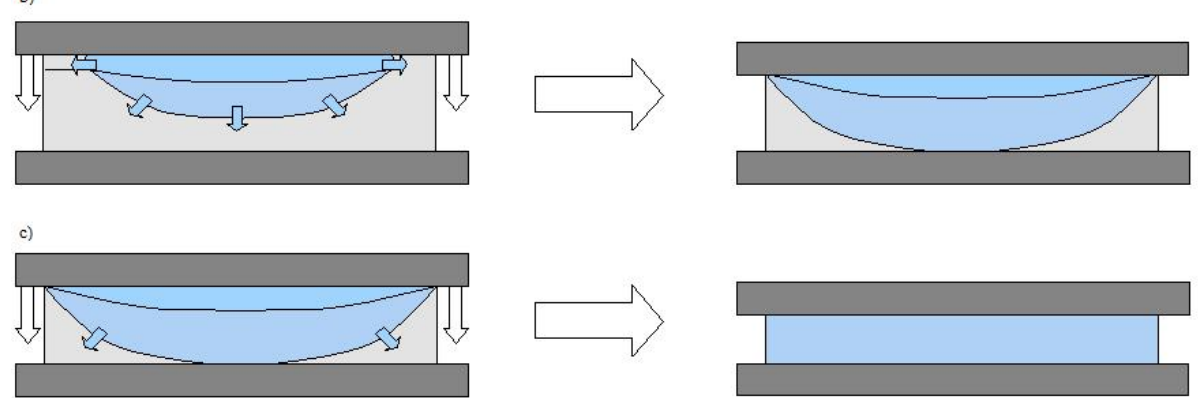

Figure 5. Three stages of CRTM process with compliant preform deforming due to the resin pressure. With this model, phases two and three may coexist within different regions of the same part.

\section{One Dimensional CRTM Modeling}

The second phase model for simple, through-thickness, one dimensional flow has been created [4]. This can be seen as a limiting case, when the resin distributes through the gap first and then infiltrates the preform through the thickness only. As demonstrated by the two-dimensional model described below, this is a case over much of the part's surface for a large range of infusion parameters. The model fully couples the preform deformation with the resin pressure and allows for deformation varying with the through-the thickness position.

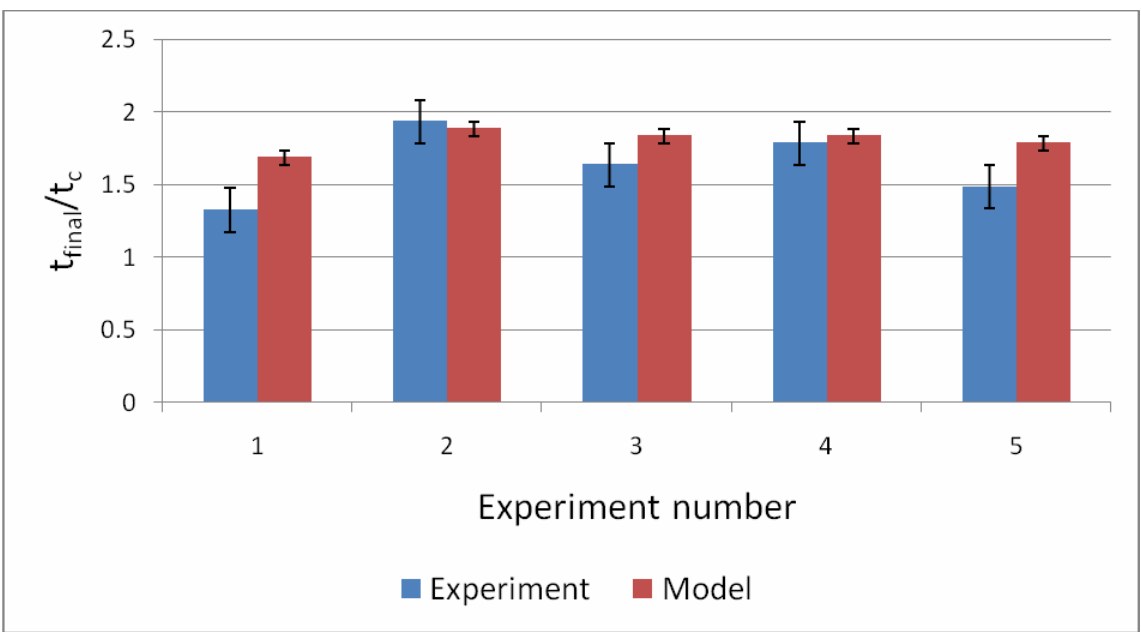

Figure 6. Prediction of normalized fill time and its comparison with experimental results for $24 \mathrm{oz}$. E-glass fabric.

The model was experimentally verified (Figure 6) and used to study the effect of process parameters on the process time. 


\section{Two Dimensional CRTM Modeling using Numerical Approach}

Two dimensional model using fully coupled flow and deformation has been developed and numerically implemented using the finite difference scheme. Paper describing the method is currently being prepared. The model allows one to simulate the CRTM infusion with full coupling between deformation and resin pressure. Convergence study has been conducted and a limited verification was performed by comparing the limiting cases, such as the case of rigid preform, with existing solutions. Figure 7 shows the pressure field within the rectangular part during the process and the part's deformation. It clearly shows non-uniform deformation at the end of Phase 2 .
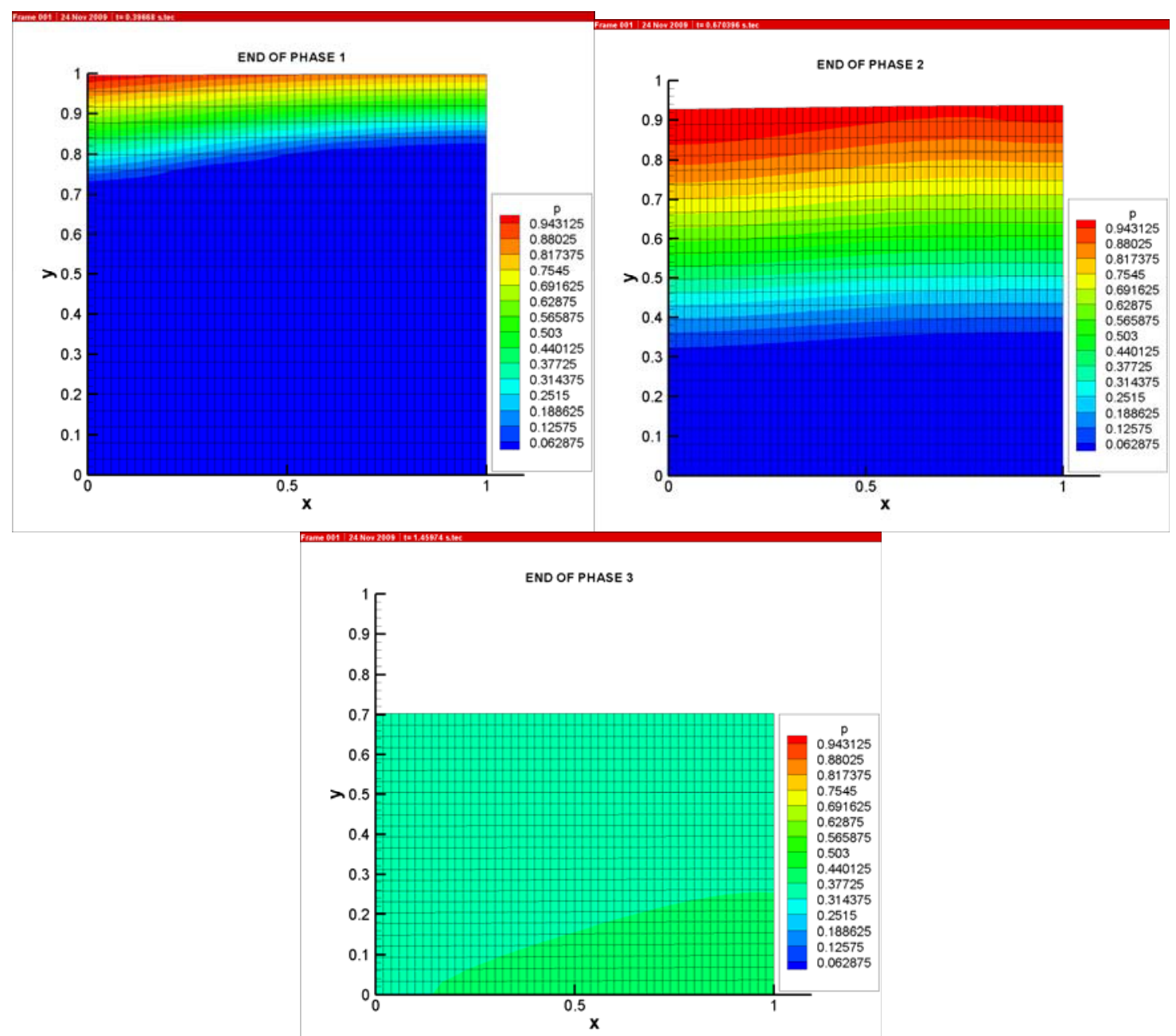

Figure 7. The pressure field and rectangular part shape during the CRTM infusion.

Dimensions and pressures are non-dimensionalized.

The model was used to study the influence of various parameters on the flow development during the CRTM process. This not only provides insight into the process dynamics, but also allows one to ascertain whether the more restricted models described above can be used to model the infusion with sufficiently accurate results. 


\section{CRTM Process Experimental Analysis}

The process has been extensively characterized using laboratory-scale experiments. First, the experiments provide insight into the process physics which shed light on what assumptions seem to be warranted. Second, the analytical and numerical description of the process requires the characterization of preform material under the conditions similar to the actual CRTM process. Finally, the results from the derived numerical models require experimental validation.

\section{Preform Characterization}

Apart from resin viscosity, two other important preform material parameters enter directly to describe the process. These are the permeability of the fabric and compaction stress experienced by the fabric when compressed. They both depend on deformation, described preferentially by the fiber volume fraction rather than some strain measure as is commonly employed in other fields.

\section{Measuring Compaction Curves and Permeability}

To measure the relation between the preform fiber volume fraction and the compacting force we used a device consisting of two parallel circular plates in an Instron machine (Figure 8). The preform fabric is cut into circular shape and stacked between these plates. Then, the compaction cycle is initiated by moving the upper plate down in a controlled manner with the instron and the force-displacement relation is recorded.

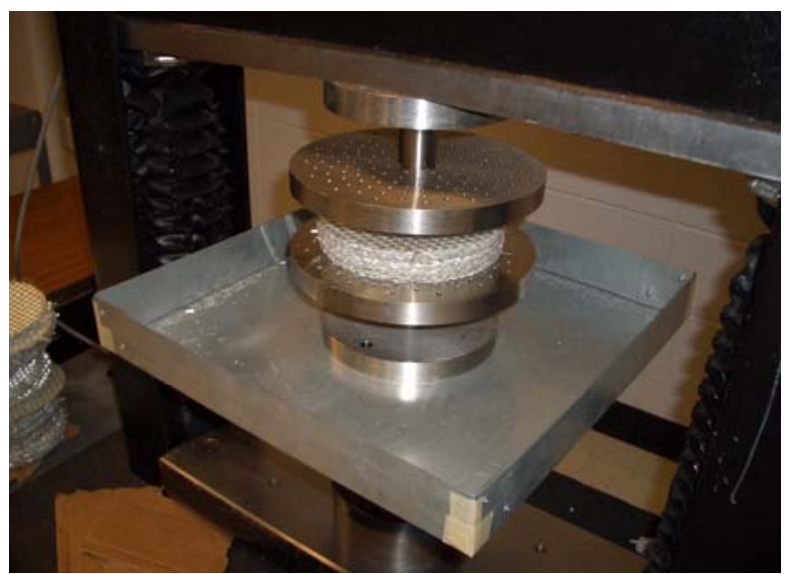

Figure 8. Experimental setup to determine the relation between compacting force and the fiber volume fraction

As the compaction behaves differently when fabric is dry as compared to when it is saturated by resin, two sets of experiments must be conducted for each preform type, dry and wet. In the wet experiment, the fluid is squeezed out of the preform, which generates extra transient compaction force. To minimize this viscous resistance of fluid, the perforated plates can be used as shown in Figure 8. Moreover, the viscous resistance can be determined by comparing experiments with solid and perforated plates and in-plane 
preform permeability evaluated using these data, providing both compaction stress and permeability from a single experimental data set (Figure 9). The through the thickness permeability must still be evaluated independently by standard methods. The experimental results show significant variability (particularly for the P4 preform) and statistical processing is necessary.
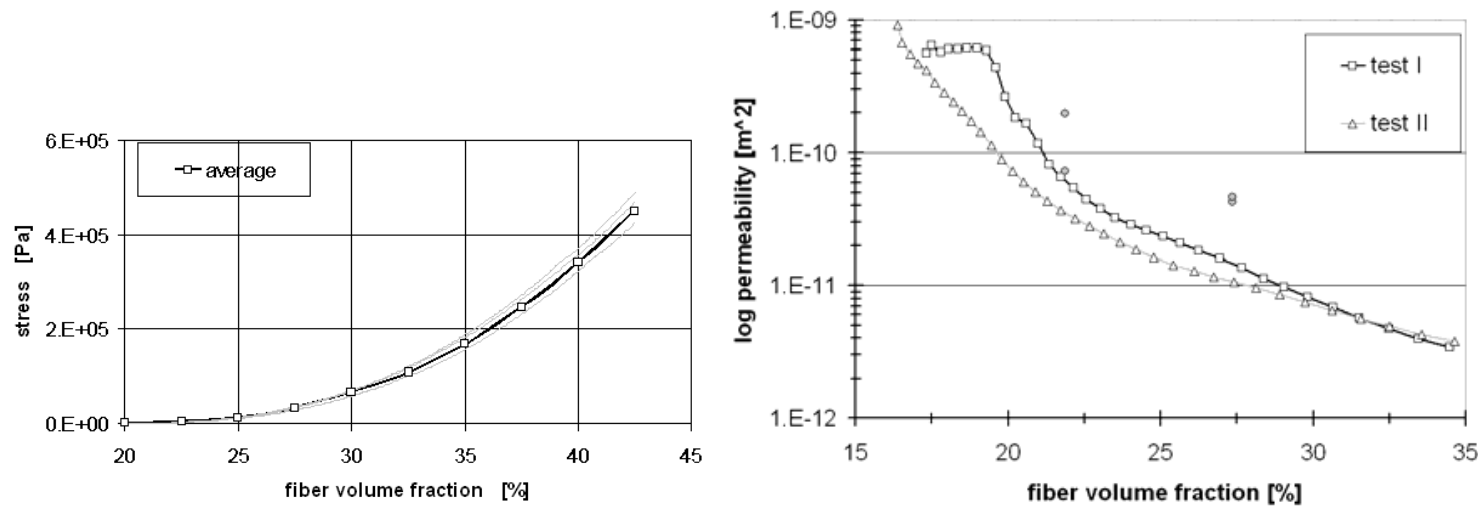

Figure 9. Dependence of compacting stress and in-plane permeability of P4 preform on fiber volume fraction.

\section{Experimental CRTM Setup}

The setup to perform experiments with the CRTM process in laboratory settings is important for two reasons. First, it allows one to explore the process and verify assumptions made. Second, it provides a tool to compare the results with the numerical model predictions.
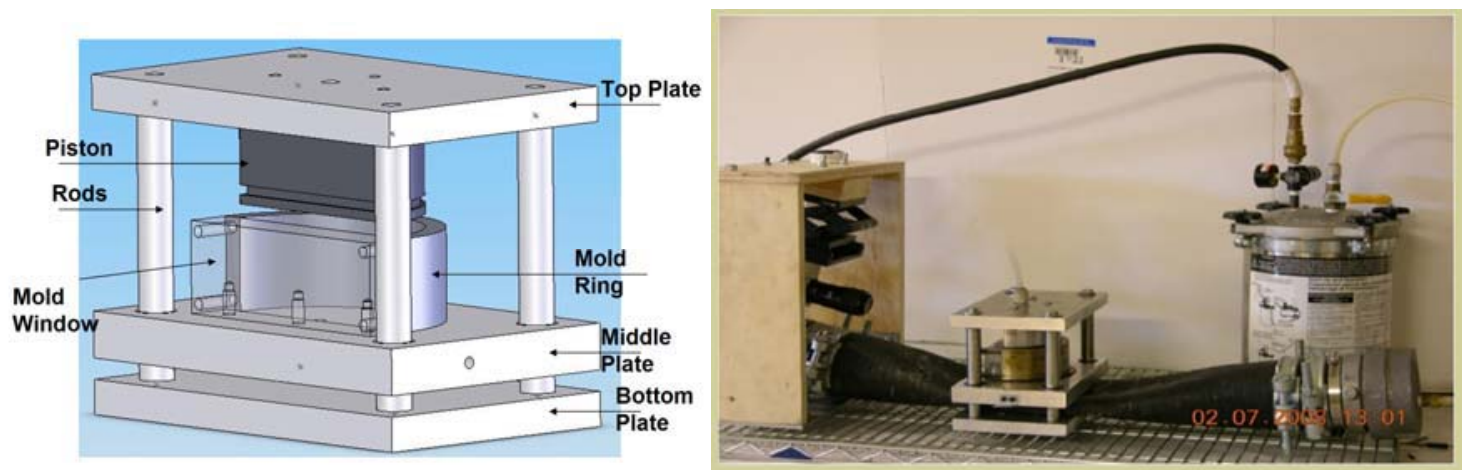

Figure 10. The first laboratory CRTM setup - schematics and implementation.

Two experimental CRTM stations were developed. The initial experimental setup (Figure 10) consisted of semi-circular mold with the straight wall made of acrylic. The compression was driven by the pressurized hose as seen in Figure 10. The flow progression could be observed through the acrylic wall (Figure 11). This device was used mostly for exploratory experiments and proved very useful. However, several issues soon became apparent. First, the compaction force varied with the displacement and lacked control. Second, the mold shape did not allow to infuse a long, slender part which is common in many applications. Also, the observation provides a view of the edge of the 
part, but due to racetracking issues at this wall it would be preferable to have some instrumentation to provide resin arrival information in other locations.
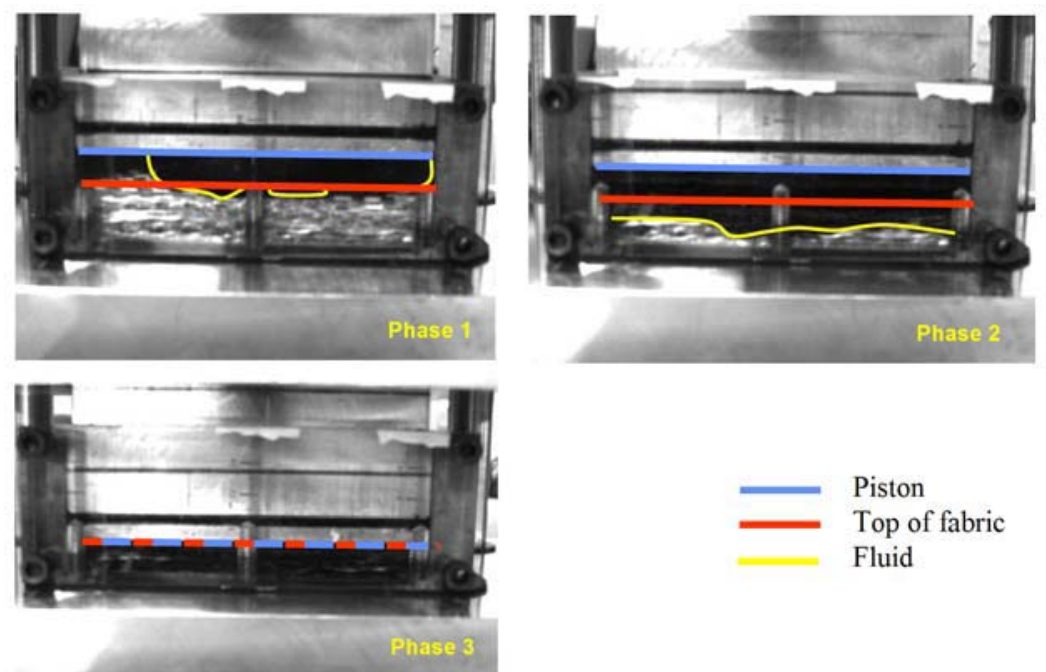

Figure 11. The CRTM infusion as seen through the mold window.

To address these issues, a different apparatus was designed and manufactured (Figure 12). This one was compacted by the piston, providing excellent control over load/displacement and allowed infusion of long thin rectangular parts to study the infusion into such a structure and compare it with the analytical work described above, as the part shape can be changed by placing inserts in the mold. The mold also provides for instrumentation to detect the fluid arrival at various locations.

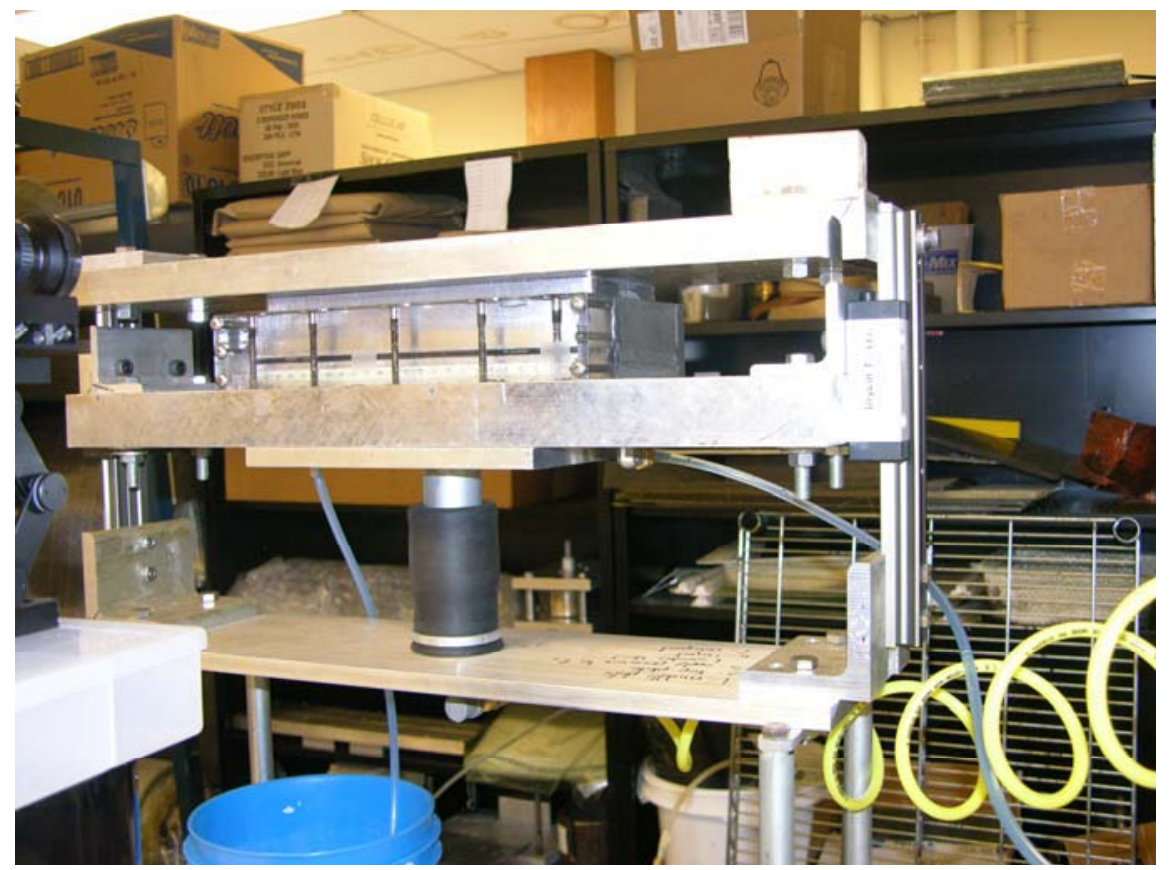

Figure 11. The second generation laboratory CRTM setup. 


\section{Fundamental Findings}

- The CRTM process can be approximately modeled using the existing LCM simulation tools.

- Limiting process requirements - based on the final phase - can be estimated from simple analytic model.

- The generally accepted assumption that the preform deforms only after the tool comes in contact with it has only limited validity. In many cases, the preform deforms significantly during the second phase due to the resin pressure.

- The preform deformation during second phase can be described using experimental preform characterization and simple conservation relations.

- One dimensional model of the second phase has been solved and experimentally verified. This model allows insight in identifying parameters that influence the process during this phase.

- The process performance tends to improve with applied pressure as one might intuitively expect, however at a certain point the improvements become negligible. It should be possible to find an optimal process parameters to balance the performance and cost for any individual application.

- Two dimensional model of the entire process allowing for the non-uniform deformation was created. A numerical code was written to create a simulation of this model without any questionable assumptions, but currently the solution is limited to the simple solution domain only (rectangular part).

- The compaction stress dependence on fiber architecture and fiber volume fraction which depends on the presence of the resin in the preform. Two measurements must be performed for any preform and, in the wet preform, the resin viscous resistance must be minimized by utilizing the perforated compaction plates.

- The in-plane permeability of the preform can be determined from wet compaction experiments as a function of fiber volume fraction.

- Two laboratory CRTM setups were developed to perform exploratory experiments and experimental verification of the numerical predictions.

\section{References}

1. "Modeling flow in compression resin transfer molding for manufacturing of complex lightweight high-performance automotive parts," Simacek, Pavel; Advani, Suresh G.; Iobst, Stanley A., Journal of Composite Materials, v 42, n 23, p 2523-2545, December 2008

2. "Process analysis of compression resin transfer molding," Bhat, P.; Merotte, J.; Simacek, P.; Advani, S.G.,Composites Part A: Applied Science and Manufacturing, v 40, n 4, p 431-41, April 2009

3. "Flow analysis during compression of partially impregnated fiber preform under controlled force," Merotte, Justin; Simacek, Pavel; Advani, Suresh G., Composites Science and Technology, v 70, n 5, p 725-733, May 2010 
4. "Resin flow analysis with fiber preform deformation in through thickness direction during Compression Resin Transfer Molding," Merotte, Justin; Simacek, Pavel; Advani, Suresh G., Composites Part A: Applied Science and Manufacturing, v 41, n 7, p 881-887, July 2010 
Final Report for Period: 09/2008 - 08/2009

Principal Investigator: Smith, Douglas E.

Organization: U of Missouri Columbia

\section{Submitted By:}

Smith, Douglas - Principal Investigator

Title:

Incorporating Higher Order Tensors in the Computation of Polymer Composite Mechanical Properties

\section{Senior Personnel}

\section{Project Participants}

Name: Smith, Douglas

Worked for more than 160 Hours: Yes

Contribution to Project:

Dr. Smith was the PI on project who was responsible for project administration, theoretical development and numerical implementation. He worked with graduate and undergraduate researchers to develop the necessary programs and procedures.

\section{Post-doc}

Name: Jack, David

Worked for more than 160 Hours: Yes

\section{Contribution to Project:}

David Jack worked as a Post-Doctoral researcher on this project to develop a statistical fiber placement program that was used to define the position and orientation of individual fibers in the representative volume element. David also developed a new fiber interaction diffusion model based on fiber/fiber collisions that may be used to predict the fiber orientation state during processing. David is now a tenure track Assistant Professor in Mechanical Engineering at Baylor University.

\section{Graduate Student}

Name: Grimshaw, Matthew

Worked for more than 160 Hours: Yes Contribution to Project:

Matt's work as a graduate research assistant includes the development of a 3D voxel-based finite element method for evaluating the elastic properties of polymer composites.

Name: Jack, David

Worked for more than 160 Hours: Yes Contribution to Project:

David's work as a graduate research assistant involved the development of statistical methods for short fiber composite property prediction and analytical work in property evaluation. Following graduation from $\mathrm{MU}$ with a $\mathrm{PhD}$ in Mechanical Engineering in December 2006, David worked as a Post-doc researcher at MU, and a Research Faculty at Florida State University before joining Baylor Univeristy in August, 2009 as a tenure-track Assistant Professor in Mechanical Engineering.

Name: Caselman, Elijah

Worked for more than 160 Hours: Yes Contribution to Project:

Elijah worked as a graduate research assistant to develop a representative volume finite element computer model where his focus was on defining the appropriate periodic boundary conditions, validating the modeling approach, and developing a Gauss-Point based material model for finite element analysis. He graduated with an MS in Mechanical Engineering from MU in December 2007 with the thesis entitled 'Elastic Property Prediction of Short Fiber Composites Using a Uniform Mesh Finite Element Method'.

Name: Joseph, Middleton

Worked for more than 160 Hours: Yes 


\section{Contribution to Project:}

Joe worked as a graduate research assistant to further develop the representative volume finite element computer modeling method. This work focused on defining long fiber geometries from scanned slices of an actual composite which was obtained from Oak Ridge National Laboratory. Joe computed 3D elastic properties for a long fiber polymer composite. He graduated with an MS in Mechanical Engineering in August 2008 with the thesis entitled 'Elastic Property Prediction of Long Fiber Composites Using a Uniform Mesh Finite Element Method'.

\section{Undergraduate Student}

Technician, Programmer

\section{Other Participant}

\section{Research Experience for Undergraduates}

Name: Arnone, Joshua

Worked for more than 160 Hours: $\quad$ Yes

Contribution to Project:

Joshua worked on this project as part of his undergraduate Honors research in the Mechanical and Aerospace Engineering (MAE) Department. He adapted the composite materials constitutive model from the short fiber composites work to simulate the fiber/matrix response in patient-specific intervertebral disk finite element models. Josh was supported under an REU supplement to the grant. Following graduation with a BS degree from MAE in December, 2007, he began his PhD study in our department. He is currently pursuing a $\mathrm{PhD}$ in Mechanical Engineering at MU.

Years of schooling completed: Other

Home Institution: Same as Research Site

Home Institution if Other:

Home Institution Highest Degree Granted(in fields supported by NSF): Doctoral Degree

Fiscal year(s) REU Participant supported: $\quad 2007$

REU Funding: REU supplement

\section{Organizational Partners}

\section{US Department of Energy (DOE) - Energy Technology Laboratory}

The US Department of Energy 2010 FreedomCar program provided partial support for this project through NSF DMI. They also made available computational resources and data from their testing that is related the goals and tasks of this research. A project Kick-off meeting was held in August 2005 to initiate the collaborative effort and a project review meeting was held in Troy, MI, in March, 2008.

As part of this collaboration, reports on research progress were exchanged between the PI and personnel at the Pacific Northwest National Laboratory (PNNL) which includes reports, journal articles, test data and PowerPoint presentations.

During the term of this project we were in contact with Dr. Ba Nghiep Nguyen at Pacific Northwest National Laboratory (PNNL) where we exchanged research results and discussed the direction of the research. Detailed information was provided to Dr. Nguyen on the statistical properties of short fiber composites. In addition, we have shared results from a new fiber orientation diffusion model that has been shown numerically to delay the amount of alignment in uniaxial and shear flows. A new NSF proposal was submitted for the Feb. 2007 unsolicited proposal cycle on a new approach to fiber interaction modeling where test data and computer resources from PNNL will be leveraged to fulfill the goals of the research. A new 3 year project entitled 'Phenomenological-Based Constitutive Model and Simulation of Fiber Interaction for Short Fiber Composites Processing' was funded by NSF beginning 9/1/2007 with David A. Jack (Baylor University) and Stephen Montgomery-Smith (MU) as Co-PIs.

The PI and Post-Doctoral Researcher David A. Jack visited PNNL in Richland, WA, on May 8, 2007 to exchange research progress. PNNL researchers visited include Dr. Ba Ngiep Nguyen and Dr. Mark Smith. 
provided on the effect of closure approximation on computed elastic properties, statistical evaluation of elastic properties computed from fiber orientation distributions, and our finite-element based approach for computing elastic mechanical properties for short and long fiber composites. All projects supported under the DOE-NSF long fiber composites research initiative were presented, and several DOE and industrial engineers were in attendance.

\section{Other Collaborators or Contacts}

Test data has been obtained from Dr. Charles L. Tucker III at the University of Illinois at Urbana-Champaign. These fiber orientation measurements are being used to assess the validity of our new fiber interaction diffusion model. In addition, a fiber placement program was obtained from Dr. Tucker to make it possible to numerically generate cubes of short fibers for elastic property simulation work.

An image file stack of long fiber composite scans were obtained from Vastimil Kunc at Oak Ridge National Laboratories. These image files are being used to extract the geometric definition of a typical long fiber composite sample in a format suitable for mechanical characterization using methods developed in this project. Data obtained from the sample will serve as input to our finite element based elastic property evaluation program.

\section{$\underline{\text { Activities and Findings }}$}

\section{Research and Education Activities:}

The main objective of this research was to develop a predictive capability that evaluates the mechanical properties of short and long \&\#64257; ber reinforced polymer composites from higher-order orientation tensors. This research developed an automated three-dimensional finite element procedure for predicting the effective mechanical properties of a fiber suspension from its orientation distribution and the mechanical properties of the constituent materials. The work also included evaluating fiber configurations using micro-CT imaging techniques and developing a computational approach for generating fiber samples numerically from polymer melt flow simulation results. As part of this research, a Monte Carlo simulation procedure was used to assess the statistical nature of the predicted properties.

Fiber reinforced polymer composites are the material of choice in numerous engineering applications, due in part to their superior strength to weight ratio. This is especially true for long- and short-fiber composite products, which also benefit from extremely versatile manufacturing methods such as injection molding. As a result, there continues to be a major effort to incorporate more fiber reinforced polymers in commercial products, particularly in the US automotive industry where future vehicles must have reduced weight, emissions, and fuel consumption. Indeed, a specific objective of the 2010 FreedomCAR program at the US Department of Energy, a co-sponsor and collaborator of this research, was to reduce the weight of an automotive structure by $50 \%$ for the same cost and durability as seen in today's products. Their aggressive goal was supported under this research project by its focus on a critical link between a fiber reinforced polymer product and its manufacturing process.

Previous work in the areas of numerical methods, composite mechanics, and fiber orientation analysis served as a basis for further development. The focus here was to:

1)Develop a model for predicting mechanical properties from higher-order orientation tensors derived from flow simulations or measurements that is not limited by the material symmetry requirements imposed by current models.

2)Develop an automated three-dimensional voxel-based finite element modeling technique that may be used to predict the effective mechanical properties of a fiber suspension.

3)Integrate Micro-CT derived fiber orientation states to illustrate the applicability of the proposed approach on production hardware to be obtained from Visteon Corporation

4)Statistically assess mechanical property calculations to address sampling issues associated with using the proposed averaging techniques.

5)Demonstrate the proposed methodology on an industrially-relevant polymer composite product where flow simulation software is to be provided from Moldflow Corporation.

6)Develop techniques for visualizing discrete fibers and fiber suspensions in reinforced polymer composites to provide students at the graduate and undergraduate levels, as well as other composite materials researchers, insight into the microstructure of polymer composites.

Project activities as part of this research project include the following:

1)The related literature was reviewed and related information and theory was collected and applied in this study.

2)A Monte Carlo simulation method was developed for evaluating the statistical nature of elastic mechanical properties of short fiber 
composites. These computations are based on sample fiber sets defined by orientation distribution functions in homogeneous and non-homogeneous flow fields.

3)Elastic mechanical properties were derived from fiber orientation tensors that are based on the expected values of the compliance tensor for random individual fibers.

4)The statistical nature (including the mean and standard deviation) of the elastic mechanical properties were derived from fiber orientation tensors and fiber distribution functions.

5)A voxel-based finite element procedure was developed for evaluating the mechanical properties of a set of short fibers in a polymer matrix. This work included the development of a representative volume element and the appropriate boundary conditions and a unique elemental strain-displacement relationship.

6)A curve fitting procedure was developed for evaluating elastic properties for various material symmetries including general anisotropic, orthotropic, transversely isotropic, and isotropic.

7)A computational verification procedure was used to demonstrate the accuracy of the voxel-based finite element approach for single fibers within a polymer matrix.

8)The voxel-based finite element method was applied to predict elastic mechanical properties of composites with continuous straight fibers, discontinuous aligned fibers, and randomly oriented short fibers.

9)A program was developed to define the spatial location and orientation of a set of fibers from fiber size and aspect ratio, and a fiber orientation distribution function.

10)A new fiber interaction model was developed to simulate fiber/fiber collisions in a manner that is suitable for use in an injection molding simulation program.

11)A composite materials-based model for the fibrous annulus of the human intervetral disk was developed. This is being performed as part of the REU supplement to this grant.

12)The voxel-based finite element method developed in this research was applied to long fiber composite samples obtained from Oak Ridge National Laboratories.

\section{Findings:}

The most significant finding in the research deals with the relationship between fiber orientation tensors and the resulting elastic mechanical properties of short fiber composites. Nearly two decades ago, Advani and Tucker presented an equation for computing a stiffness (or compliance) tensor from a 4th order fiber orientation tensor and the constitutive properties of the fiber and matrix. This earlier work used the unidirectional elastic properties of several single fibers, each having a pre-selected orientation, to arrive at the final result which was given with little derivation. In our work, a rigorous derivation was performed to evaluate the same results from the statistical expectation of single fiber mechanical properties where the distribution of fibers is defined by the fiber orientation distribution function. Unidirectional properties of single fibers are rotated into a common reference frame where the rotation tensor is defined by directions that follow the orientation distribution function itself. As a result, we are now able to compute mechanical properties from either orientation tensors or fiber orientation distribution functions. We have also been able to show that orientation tensors having an order higher than four do not contribute to the expectation value when the commonly used Fourier basis functions are employed. This derivation reduces to the Advani and Tucker form when it is assumed that fibers do not interact under stress and strain in the final composite.

Using a similar derivation technique, we also derived, for the first time, a means to quantify the statistical variability in the elastic properties of a short fiber composite. As a result, we can now compute the variance of a characteristic volume of fibers from the fiber orientation distribution function or higher order tensors. It is shown that the variance requires orientation tensors through 8th order when orientation tensors are used in place of an actual distribution function.

In addition, a Monte Carlo simulation method has been developed to assess the statistical nature of short fiber composites that may be described by fiber orientation distribution functions. In this work, sample sets of fibers are defined by a representative cell size, fiber size, and volume fraction. For each sample set, elastic mechanical properties are computed from the calculations described above. The results of numerous sample sets are then combined to obtain the mean and standard deviation of the predicted elastic properties. The calculations have been performed for selected fiber orientation distribution functions, as well as those that are computed from simple homogeneous and non-homogeneous flows. Results have validated those derived from the orientation function expectation described above. We have also compared the results of these calculations when various closure approximations are employed.

A voxel-based finite element (FE) formulation has also been developed to compute the elastic mechanical properties for both short and long fiber composites. This approach divides a representative volume element into cube-shaped finite elements. The number of elements and number of degrees of freedom are significantly reduced by increasing the element size while adding additional integration points to capture the spatial variation in mechanical properties within a given element. The model was developed to run in the general purpose finite element program ABAQUS where the user defined element subroutine (UEL) is employed to add this additional capability. The representative volume element includes the appropriate periodic boundary conditions and elemental strain-displacement relationships so that the results are representative of a 
larger domain. Verification simulations have been done to show that we can compute orthotropic elastic constants that are in agreement with classical theories for isotropic materials. Numerous 2D and 3D examples from the literature have been used to validate this new modeling approach. The method has successfully been applied to continuous straight fiber composites, short aligned fiber composites, and randomly oriented short fiber composites. Computed results agree well with literature data for the aligned and continuous fiber models, and for random fiber models with low volume fractions. Results deviate from analytical models as the fiber volume fraction increases, as expected based on literature data.

Our voxel-based finite element approach has also been applied to long fiber composites. To perform these analyses, the fiber geometry is defined by values from the voxels of micro-CT images in a manner that is suitable for our modeling approach. Short fiber geometries were defined analytically by generating multiple individual straight fibers within a characteristic volume. Long fibers are not as straight-forward, since they are typically long enough to pass completely through an RVE of reasonable size. As a result, the short fiber analytical approach described above becomes inappropriate. Therefore, to properly represent long fiber geometries, we used micro-CT scanned data obtained from Oak Ridge National Laboratory for a 1 by 1 by $1.5 \mathrm{~mm}$ sample with 2 micron resolution. Various image processing techniques such as cropping, dilation, contraction, and filtering methods were applied to obtain a transformed data set that was suitable for our voxel-based FE approach. The image data was then used to assign elastic properties at each integration point in the finite element model. Periodic boundary conditions were applied and mechanical properties were computed in a manner that is similar to that used in the short fiber analysis. Results provided reasonable values for the long fiber composite, and identified resin-rich regions near the center of the sample.

The Monte Carlo simulation program described above was enhanced to be used with our voxel-based finite element modeling method. A molecular dynamics simulation was implemented to adjust the position of fibers so that they do not overlap in space while maintaining a predefined orientation distribution function. Preliminary results were obtained for mechanical properties that agreed well with expected values.

Our new fiber interaction model was implemented in a program that evaluates fiber orientation tensors for fiber distributions in an arbitrary flow. Limits on the parameters contained in the model were established and limitations of the approach were identified. Fiber orientation predictions were compared with test data obtained from PNNL. Results show a significant improvement in elongational flows, but much less effect in shear-dominated flows. Further development is needed to obtain an improved accuracy in shear flows.

As part of the REU supplement to this grant, an undergraduate student developed a finite element modeling approach for representing the fibrous annulus of the intervertebral disc as a long fiber composite material. All previous approaches 'stitch' in bar elements within a matrix of solid bricks to represent the fibers. This earlier approach is tedious and is only appropriate for simple geometries. Our approach rotates a transversely isotropic elasticity tensor defined by the matrix and fiber properties into the direction of the disk fibers. As a result, each element has its own fiber orientation and elastic properties. This approach will make it possible to model the actual disk geometry which is far more complex than that used in earlier analyses. Our approach is made possible by leveraging the short and long fiber composites work described above.

\section{Training and Development:}

David A. Jack: Participation 9/05 ? 12/06. David graduated with a Ph.D. in Mechanical Engineering in December 2006. He also earned an MS in Applied Mathematics in December 2006 as well.

David A. Jack: Participation 4/07 ? 7/07. David joined this project as a Post-Doctoral researcher in April 2007. He was instrumental in developing the fiber orientation statistical model. Following his Post-Doc, David joined the Industrial and Manufacturing Engineering Department at Florida State University as a Research Assistant Professor. In August, 2009, he started as a tenure-track Assistant Professor at Baylor University.

Matthew T. Grimshaw: Participation 1/06 ? 4/07. Matt is a MS student at MU in Mechanical Engineering who has completed much of his course work towards his degree. Matt did not complete his thesis research project and took a job in industry in 2008.

Elijah C. Caselman: Participation 1/07 ? 12/07. Elijah graduate with an MS in Mechanical Engineering in December, 2007.

Joseph E. Middleton: Participation 1/08 ? 8/08. Joe completed his research and graduated with an MS in Mechanical Engineering in August, 2008.

Joshua C. Arnone: Participation 6/06 ? 12/07. Josh participated as an undergraduate honors research student in Mechanical Engineering at MU funded through an NSF REU supplement to this project. He graduated with a BS in Mechanical Engineering in December 2007 and is currently enrolled in the Ph.D. program here at MU in Mechanical Engineering. 


\section{Outreach Activities:}

\section{Journal Publications}

D.A. Jack and D.E. Smith, "Elastic Properties of Short-Fiber Polymer Composites, Derivation and Demonstration of Analytical Forms for Expectation and Variance from Orientation Tensors", Journal of Composite Materials, p. 277, vol. 42:3, (2008). Published,

David A. Jack and Douglas E. Smith, "The Effect of Fiber Orientation Closure Approximation on Mechanical Property Prediction", Composites Part A., p. 975, vol. 38, (2007). Published,

\section{Books or Other One-time Publications}

David A. Jack, "Advanced Analysis of Short-Fiber Polymer Composite Material Behavior with Higher-order Orientation Tensor Closure Methods", (2006). Thesis, Published

Bibliography: University of Missouri

Elijah C. Caselman, "Elastic Property Prediction of Short Fiber Composites Using a Uniform Mesh Finite Element Method", (2007). Thesis, Published

Bibliography: University of Missouri

Joshua Arnone, "Three Dimensional Composite Material Modeling Approach for the Intervertebral Disc", (2007). Undergraduate Honors Thesis, Published

Bibliography: Mechanical and Aerospace Engineering Department, University of Missouri

Joseph Middleton, "Elastic Property Prediction of Long Fiber Composites Using a Uniform Mesh Finite Element Method", (2008). Thesis, Published

Bibliography: University of Missouri

\section{Web/Internet Site}

\section{Other Specific Products}

\section{Product Type:}

\section{Conference Paper and Poster Presentation}

\section{Product Description:}

D.E. Smith, M.T. Grimshaw, and D.A. Jack, 'Incorporating Higher Order Tensors in the Computation of Polymer Composite Mechanical Properties', Poster presentation at the 2006 NSF Design, Service and Manufacturing Grantees and Research Conference, St. Louis, Missouri, July 24-27, 2006.

\section{Sharing Information:}

Work included as poster presentation and conference proceedings paper.

\section{Product Type:}

\section{Conference paper and poster presentation}

\section{Product Description:}

D.E. Smith and D.A. Jack, 'Computing Elastic Material Properties of Short Fiber Composites via Monte-Carlo Simulation', Poster presentation at the 2008 NSF Design, Service and Manufacturing Grantees and Research Conference, Knoxville, Tennessee, January 7-10, 2008.

\section{Sharing Information:}

Work included as poster presentation and conference proceedings paper. 


\section{Product Type:}

\section{Refereed confernce paper and presentation}

\section{Product Description:}

Jack, D.A. and D.E. Smith. 'A Statistical Method to Obtain Anisotropic Material Proper-ties from the Orientation Distribution Function for Short-Fiber Polymer Composites.' In proceedings of 2005 ASME IMECE, Orlando, Florida, November, 2005.

\section{Sharing Information:}

Work included as podium presentation and conference proceedings paper.

\section{Product Type:}

\section{Refereed conference paper and presentation}

\section{Product Description:}

E.C. Caselman and D.E. Smith, 'A Multi-Gauss Point Finite Element Approach for the Predicting Elastic Properties of Short Fiber Composites,' Accepted for the Annual Meeting of the Polymer Processing Society, PPS-24, Salerno (Italy),15 - 19 June 2008

\section{Sharing Information:}

Work included as podium presentation and refereed conference paper.

\section{Product Type:}

\section{Refereed conference paper and presentation}

\section{Product Description:}

D.E. Smith and D.A. Jack. 'Neural Network-based Closure Approximation for Short-Fiber Suspension Simulations,' Accepted for the Annual Meeting of the Polymer Processing Society, PPS-24, Salerno (Italy),15 - 19 June 2008.

\section{Sharing Information:}

Work included as podium presentation and conference proceedings paper.

\section{Product Type:}

\section{Accepted Journal Article}

\section{Product Description:}

The following journal article has been accepted for publication in Polymer Composites: D.A. Jack, B. Schache, and D.E. Smith, "Neural Network-Based Closure for Modeling Short Fiber Suspensions".

\section{Sharing Information:}

This journal article has been accepted for publication in Polymer Composites and is availabe as Early View at http://www3.interscience.wiley.com/cgi-bin/fulltext/122598276/PDFSTART.

\section{Product Type:}

\section{Conference Proceedings}

\section{Product Description:}

D.E. Smith, "Computing Elastic Properties of Short- and Long-Random Fiber Composites Using a Multi-Gauss Point Finite Element Method", American Society for Composites, Canadian Association for Composite Structures and Materials, 24th Technical Conference, University of Delaware, Newark, DE, September 15, 2009.

\section{Sharing Information:}

Paper presented as a podium presentation, and paper published in conference procedings.

\section{Product Type:}

\section{Conference Paper and Poster Presentation}

\section{Product Description:}

D.E. Smith, E. Caselman, and J. Middleton, "Computing Elastic Properties of Short- adn Long-Random Fiber Composites Using a Multi-Gauss Point Finite Element Method". Proceedings of 2009 NSF Engineering Research and Innovation Conference, Honolulu, HI, July, 2009.

\section{Sharing Information:}

Conference paper and poster presentation.

\section{Contributions}




\section{Contributions within Discipline:}

Specific contributions within discipline to date include: 1) the role of closure approximation in the computation of elastic properties, 2) a method to assess the statistical properties of the elastic mechanical properties of short fiber suspensions, 3 ) a derivation to support current calculation procedures for the mechanical properties of short fiber suspensions that exposes the lack of fiber-fiber interactions in these evaluations, 4) a multi Gauss point finite element method with consistent displacement interpolation procedure, 5) computation of elastic properties for continuous, short aligned, short random, and long random fiber composites.

\section{Contributions to Other Disciplines:}

The intervertebral disk model provides a new approach for the biomechanical simulation of the spine. This approach makes it possible to better represent patient specific geometries in the mechanical analysis of the spine.

\section{Contributions to Human Resource Development:}

Matthew T. Grimshaw has served as the teaching assistant for the MU course MAE3100 'Computational Methods in Engineering Analysis and Design' for the Fall 2005, Winter 2006, Fall 2006, and Winter 2007 semesters.

Elijah Caselman served as a TA for the MAE computer graphics course during the Winter 2007 semester.

David A. Jack was the instructor for a new course in MAE entitled 'Introduction to Engineering Computing' during the Winter 2007 semester.

\section{Contributions to Resources for Research and Education:}

\section{Contributions Beyond Science and Engineering:}

A presentation entitled 'Engineering Computing with Matlab' was presented to the Columbia Area Career Center (CACC), Columbia, MO, on April 15, 2009. The Columbia Area Career Center is part of the Columbia Public School District and offers adult technical courses and high school electives. This was done as part of a larger effort to familiarize high school students with the use of Matlab as a computational tool. It was planned to offer a course in Matlab for high school students at the CACC, but efforts to do this are still in the planning stages and did not result in a course offering under this grant period.

\section{Conference Proceedings}

\section{Categories for which nothing is reported:}

Activities and Findings: Any Outreach Activities

Any Web/Internet Site

Contributions: To Any Resources for Research and Education

Any Conference 


\section{Introduction}

The objective of this Interagency Agreement was to further the art of polymer science as it relates to high strength polymer and polymer composite materials. While furthering the state of the art, these developments will enable high strength, lighter weight materials to be developed for vehicle and other applications.

\section{Scope}

Grants were issued by the National Science Foundation to institutions selected under DMII/MPM program under the February 1, 2005 deadline (NSF 04-23) performing research in the areas of polymer and polymer composite structure and processing. The work encompassed mathematical modeling and experimental measurements, as well as their impact on process design.

The tasks of this Interagency Agreement corresponded to the grants which were awarded by the National Science Foundation, below:

Task 1 performed by Northwestern University - Microstructural Modeling and Synchrotron Studies of Orientation Development in Injection Molding of Liquid Crystalline Polymers

The Recipient shall investigate thermotropic liquid crystalline polymers (TLCPs) and their behavior during injection molding. New modeling strategies will be implemented to predict orientation development, and may employ polydomain descriptions of orientation to facilitate comparison with experimental orientation measurements. Experimental studies will include development and application of in situ x-ray scattering measurements in real time and ex situ synchrotron spectroscopy for 3-dimensional (3-D) surface layer characterization.

Task 2 performed by University of Massachusetts Lowell - Linking Process-Induced Properties to Thermoplastic-Matrix Woven-Fabric Composites Performance

The Recipient shall utilize an integrated analytical, experimental, and numerical effort to attain a fundamental understanding of interlayer (fabric-fabric) friction and interconnected through-thickness and in-plane compaction in woven-fabric reinforced structural composites.

A predictive model will be developed that can be used by industry to identify critical process and material parameters, thereby facilitating the greater use of lightweight composite materials in the automotive industry Capabilities of the predictive model will be demonstrated by considering a prototype part from design conception through manufacturing under a set of prescribed conditions and examining its in-service performance.

Task 3 performed by Virginia Polytechnic Institute and State University - Simulation of Injection Molding of Thermoplastics Reinforced with Fibers and Nano-particles The Recipient shall simulate the mold filling process for thermoplastic melts reinforced with short and long fibers and nano-particles of high aspect ratio using constitutive relations which allow coupling between the flow and particle orientation and to include kinematics of frontal flow. The predicted particle orientation distribution will be used 
along with micro-mechanical models to predict the stiffness variation throughout an injection molded part. The simulation will be assessed by comparing the predicted particle orientation distribution throughout an injection molded part.

Task 4 performed by University of Missouri-Columbia - Incorporation of Higher Order Tensors in the Computation of Polymer Composite Mechanical Properties

The Recipient shall develop a predictive capability that incorporates higher-order orientation tensors to evaluate mechanical properties of short fiber polymer composites. This will include, but is not limited to:

- Development of a model to predict mechanical properties of short fiber suspensions from higher-order orientation tensors;

- Implementation of an automated 3-D voxel-based finite element approach to evaluate the micromechanics of short fiber suspensions;

- Generation of three-dimensional reconstructions of actual fiber suspensions measured with Micro-CT imaging techniques and compute mechanical properties;

- Development and application of a Monte Carlo approach to assess the effect of fiber orientation sampling; and

- Demonstration of the proposed computational approach on a composite product.

Task 5 performed by University of South Carolina - Development of a Hierarchical, Structure-oriented and Stochastic Approach to Model Liquid Molding Processes The Recipient shall develop a hierarchical modeling framework for liquid molding operations that will explicitly consider the heterogeneous nature of the preform and/or fabric materials as well as the need for computational rigor and efficiency in mold filling simulations. This will include, but is not limited to:

- Development of meso-scale permeability models in terms of the statistics of micromorphological parameters which characterize the fibrous medium;

- Use of the above models to define the statistical structure and spatial continuity of the effective permeability field for saturated and un-saturated flows at a length scale consistent with a finite difference or finite element discretization as applied in CAD/CAE; and

- Establishment of practical guidelines on the amount of meso-scale information needed to unambiguously delineate the upscaled permeability field to a desired level of accuracy in filling simulations.

Task 6 performed by University of Delaware - Simulation of Compression-Resin Transfer Molding Process for Manufacturing Net Shape Structures

The Recipient shall formulate a process model for the resin flow and compressive forces during the Compression Resin Transfer Molding (CRTM) process. The model will be implemented in a Liquid Composite Molding simulation for user-friendly interface. Governing equations will be formulated and analyzed to identify key material and process parameters and to develop methods for experimental and analytic determination of these parameters. Model simplifications will be attempted using this data and governing equations. The numerical formulation will be developed and efficient algorithms will be explored to solve these equations in order to provide predictive 
modeling for flow patterns, times and forces required for successful filling and fiber deformation in the CRTM process.

Exploratory experiments will be conducted to build fundamental understanding of flowcompression coupling in the CRTM process by characterizing both the process and the materials of interest.

The simulation will be used to design the mold and process for prototype net shaped components. Existing optimization algorithms will be applied to CRTM the process to optimize the design, materials and process simultaneously for desired performance.

\section{Conclusion}

The attached appendix provides the final reports from the grantee institutions in performance of the awarded scope. 
Appendix

Final grantee reports 